\title{
ReLiability OF the QuenCH \\ PROTECTION SYSTEM For the LHC SUPERCONDUCTING ELEMENTS
}

\author{
Antonio Vergara Fernández \\ Doctor por la UPC - Mención Doctorado Europeo \\ Universitat Politècnica de Catalunya \\ Departamento de Física e Ingeniería Nuclear \\ Programa de Doctorado en Ingeniería Nuclear \\ Director de Tesis: Dr. Francisco Calviño Tavares
}

CERN. European Organization for Nuclear Research

Accelerator Technology Division

Magnets and Electrical Systems Group

Superconducting Magnet Protection Section

Supervisor: Félix Rodríguez Mateos

\author{
PhD Thesis \\ Discussion: Barcelona, November 7, 2003 \\ Sobresaliente Cum Laude
}

Prof. Xavier Ortega Aramburu - Universitat Politècnica de Catalunya

Prof. Joan Figueras Pamies - Universitat Politècnica de Catalunya

Prof. Enrico Zio - Politecnico di Milano

Prof. Luigi Palumbo - Universita di Roma "La Sapienza"

Prof. Josep Sempau Roma - Universitat Politècnica de Catalunya

Cover Design by Jerónimo Durán 



\section{to my father}

\section{to my mother}





\section{Abstract}

The huge energy stored in the Large Hadron Collider (LHC) could potentially cause severe damage when the superconducting state disappears (quench) if precautions are not taken. Most of the superconducting elements in this accelerator require protection in case of resistive transition. The reliability of the Quench Protection System will have a very important impact on the overall LHC performance.

Existing high energy accelerators were conceived as prototypes whose main objective was not the efficiency but to push the performance to the limit. The LHC will be the first large accelerator with reliability oriented design.

This thesis focuses on adapting the reliability theory and methods that are currently used by the industry and small accelerators to the protection systems of larger and more complex machines like the LHC. Two main contributions have been achieved. Firstly, the author has developed an analytical model for a general multi-state protection system, which is able to include the specific characteristics of a large superconducting accelerator. In order to provide a tool for its implementation and related computations, the simulation program RESQP (REliability Software for Quench Protection studies) has been developed. Secondly, a theoretical and experimental analysis of the LHC Quench Protection System dependability has been carried out. 


\section{List of Variables}

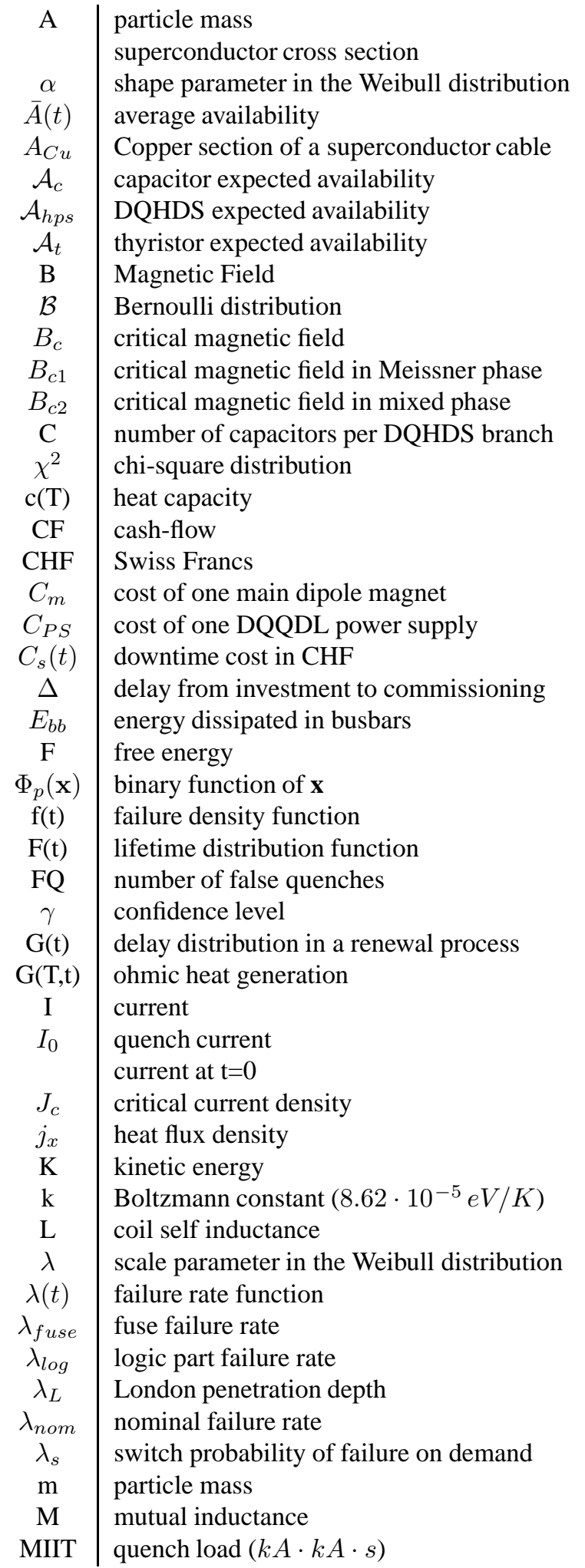

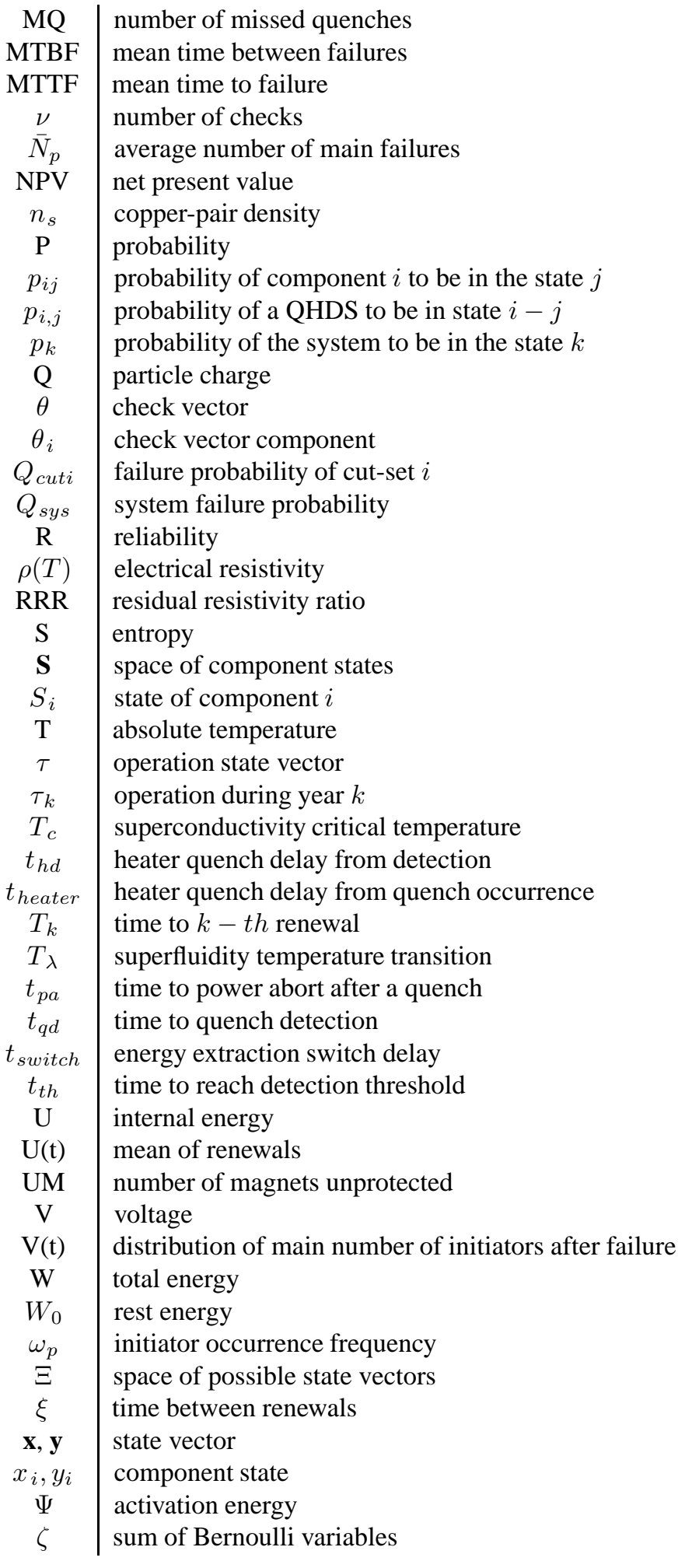




\section{Contents}

$\begin{array}{lll}\text { Abstract } & \text { v }\end{array}$

$\begin{array}{lr}\text { Introduction } & 1\end{array}$

1 The Large Hadron Collider $\quad 3$

1.1 High Energy Particle Physics Today . . . . . . . . . . . . . . . . . . 3

1.2 The LHC Project . . . . . . . . . . . . . . . . . . . . 4

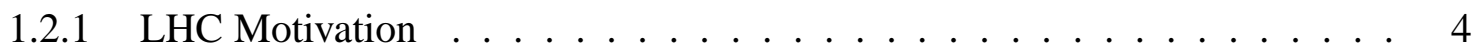

1.2.2 Technology Challenges . . . . . . . . . . . . . . . 5

1.3 LHC Layout . . . . . . . . . . . . . . . . . . . . . . . 7

2 Superconductivity and Superconducting Magnets 11

2.1 Basics of Superconductivity . . . . . . . . . . . . . . . 11

2.1 .1 History Overview . . . . . . . . . . . . . . . . . 11

2.1.2 Meissner-Ochsenfeld Effect . . . . . . . . . . . . . . . . 12

2.1.3 The Critical Magnetic Field . . . . . . . . . . . . . . . . . . 13

2.1 .4 Type II Superconductors . . . . . . . . . . . . . . . . . . . . 13

2.1.5 Hard Superconductors ........................ 14

2.2 Superconducting Accelerator Magnets . . . . . . . . . . . . . . . . . 15

2.2.1 Superconducting Materials for Accelerators . . . . . . . . . . . . . . 15

2.2.2 Stability of Superconducting Cables . . . . . . . . . . . . . . . 16

2.3 Quenches and Quench Protection . . . . . . . . . . . . . . . . 17

2.3.1 Types of Quenches . . . . . . . . . . . . . . . . . . . . . . . . . . . . . . . . . .

2.3 .2 Coil Stability . . . . . . . . . . . . . . . . . . . . . . . . . . . . . . . .

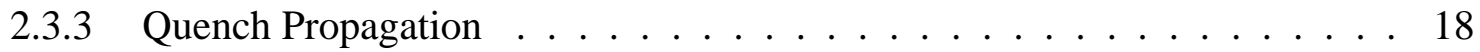

2.3.4 Protection of a string of magnets . . . . . . . . . . . . . . 19

2.4 Superfluid Helium . . . . . . . . . . . . . . . . . . . 22

3 LHC Superconducting Systems $\quad 25$

3.1 LHC Main Magnets . . . . . . . . . . . . . . . . . . . . . . . . . . . . . . . . . . . .

3.1 .1 Main Dipoles . . . . . . . . . . . . . . . . . 25

3.1.2 Main Quadrupoles and Magnets in the Insertions . . . . . . . . . . . . . 27

3.2 Corrector Magnets . . . . . . . . . . . . . . . . . . . . . . . . . . . . . . . . . . . . . . . . . . .

3.3 Busbars and Current Leads . . . . . . . . . . . . . . . . . . . . . . . . 29

3.3.1 Superconducting Busbars . . . . . . . . . . . . . . . . 29

3.3 .2 HTS Current Leads . . . . . . . . . . . . . . . . . . . . . . . . . . . . . . . .

3.4 Cryogenic System . . . . . . . . . . . . . . . . . . 30 
3.5 LHC Quench Protection System . . . . . . . . . . . . . . . . . 31

3.5.1 Protection of Main Magnets . . . . . . . . . . . . . . 32

3.5.2 Protection of the Insertion Magnets . . . . . . . . . . . . . 36

3.5.3 Protection of the Corrector Circuits . . . . . . . . . . . . . . 36

3.5.4 Protection of Superconducting Busbars . . . . . . . . . . . . . 36

3.5.5 Protection of the HTS Current Leads . . . . . . . . . . . . . . . . . . . . . . . . . . . . . . . . . . .

3.5.6 Data Acquisition and Monitoring . . . . . . . . . . . . 37

3.5.7 Radiation Tolerant Equipment . . . . . . . . . . . . . . . . . . 38

3.5 .8 Reliable Equipment . . . . . . . . . . . . . . . 38

4 Accelerator Reliability $\quad 39$

4.1 Brief Reliability History . . . . . . . . . . . . . . . . . . . . 39

4.2 Reliability Engineering . . . . . . . . . . . . . . . . . . . . . . . . . . . . . . . .

4.2 .1 Definitions . . . . . . . . . . . . . . . . 40

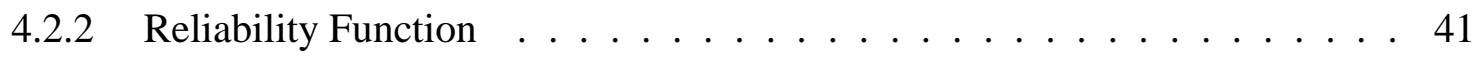

4.2.3 Typical Failure Distributions: The Bath-Curve . . . . . . . . . . . 42

4.2.4 Mathematical Modelling of Systems . . . . . . . . . . . . . . . . 45

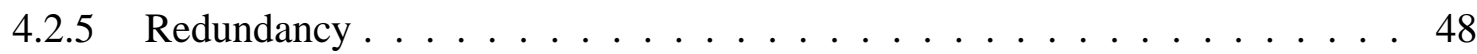

4.3 Reliability in Civil and Military Industries . . . . . . . . . . . . . . . . . . . . 50

4.4 Reliability of Existing Accelerators . . . . . . . . . . . . . . . . . . . . 51

4.5 LHC Reliability . . . . . . . . . . . . . . . . . . . . . . . . . . . . . . . . . . . . . . . . . . . .

4.6 QPS Reliability . . . . . . . . . . . . . . . . . . 56

5 Reliability in Accelerator Protection Systems 59

5.1 Protection Systems in Particle Accelerators . . . . . . . . . . . . . . . . . . 59

5.2 RESQP: Reliability Model for a Multi-State Protection System . . . . . . . . . . . 60

5.2.1 Safe and Dangerous Failure Reliability . . . . . . . . . . . . . . 60

5.2.2 Renewal Theory: from Dangerous Failure to Main Failure . . . . . . . . . 65

5.3 Sensitivity Analysis . . . . . . . . . . . . . . . . . . 68

5.4 RESQP execution . . . . . . . . . . . . . . . . . . 70

5.5 Component Reliability . . . . . . . . . . . . . . . . . . 71

5.5.1 Radiation Influence on Component Reliability _ . . . . . . . . . . . 71

5.5 .2 Other External Factors . . . . . . . . . . . . . . . . . . . 74

5.5 .3 Failure Distribution Data . . . . . . . . . . . . . 74

6 Validation and Failure Mode Analysis of the Quench Protection System 77

6.1 Component Validation . . . . . . . . . . . . . . . . 77

6.1.1 Radiation Tests on Electronic Equipment . . . . . . . . . . . . . 77

6.1 .2 Stress Tests . . . . . . . . . . . . . . . . . . . . . . . . . . . . . . . .

6.2 Protection Strategy Validation . . . . . . . . . . . . . . . . 81

6.2 .1 The LHC Test String-2 . . . . . . . . . . . . . . . . . . . . . . . . . . . . . . . . . . . . .

6.2.2 String-2 Quench Protection System . . . . . . . . . . . . . . . 82

6.2.3 Commissioning and First Quenches in String-2 . . . . . . . . . . . . . . . 84

6.3 Failure Mode Analysis . . . . . . . . . . . . . . . . . . 85

6.3.1 Quench Simulation Programs . . . . . . . . . . . . . . . 86

6.3.2 Validation and Calibration of the Models . . . . . . . . . . . . . 86

6.3.3 Simulated Circuit Model . . . . . . . . . . . . . . . 87 
6.3 .4 Nominal Operation . . . . . . . . . . . . . . . . . . 88

6.3 .5 Diode Failure . . . . . . . . . . . . . . . . . . 90

6.3 .6 Energy Extraction Failure . . . . . . . . . . . . . . . . 93

6.3.7 Quench Heater Supply Failure . . . . . . . . . . . . . . . . . . . . 96

6.3 .8 Power Abort Failure . . . . . . . . . . . . . . . . . . . . . 97

6.3 .9 Quench Detector Failure . . . . . . . . . . . . . . . . . . 99

6.3 .10 Fault Tree Analysis (FTA) . . . . . . . . . . . . . . . . 100

7 Reliability of the LHC Quench Detectors 103

7.1 Main Magnet Local Quench Detectors (DQQDL) . . . . . . . . . . . . . . . . . . . . . . . . . . . . .

7.1 .1 Detector Design . . . . . . . . . . . . . . . 103

7.1 .2 Failure Modes . . . . . . . . . . . . . . . . . . . . . . . . . . . . . . . . . . . . .

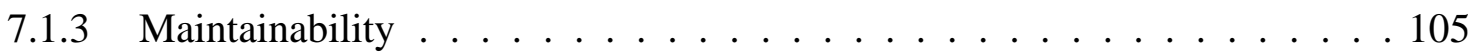

7.1.4 Reliability Data of the Components . . . . . . . . . . . . . . 107

7.1 .5 Studied Topologies . . . . . . . . . . . . . . . . . . . . . . . . . . . . . . . . . . . . .

7.1 .6 Sensitivity Analysis . . . . . . . . . . . . . . . . 112

7.1.7 Powering and False Quenches . . . . . . . . . . . . . . . . 112

7.2 Inner Triplet (DQQDT) and Insertion Magnet (DQQDI) Quench Detectors . . . . . 117

7.2.1 Detector Design . . . . . . . . . . . . . . . 118

7.2.2 Reliability Data of the Components . . . . . . . . . . . . . 119

7.2.3 Studied Topologies . . . . . . . . . . . . . . . . . . . . . . . . . . . . . . . . . . . . . .

7.3 Corrector Magnet Quench Detectors (DQQDG) . . . . . . . . . . . . . 123

7.3.1 Quench Rate and Failure Costs . . . . . . . . . . . . . . 123

7.3.2 Detector Design . . . . . . . . . . . . . . . . . . . . . . . . . . . . . . . . . . . . . . . .

7.3.3 Studied Topologies . . . . . . . . . . . . . . . . . 125

7.4 Superconducting Current Lead Quench Detectors (DQQDC) . . . . . . . . . . 127

7.4.1 Detector Design . . . . . . . . . . . . . . . . 127

7.4.2 Reliability Data of the Components . . . . . . . . . . . . 127

7.4 .3 Studied Topologies . . . . . . . . . . . . . . . 127

8 Reliability of the LHC Quench Heater Power Supplies 131

8.1 The LHC Quench Heater Power Supplies (DQHDS) . . . . . . . . . . . . . . . . . 131

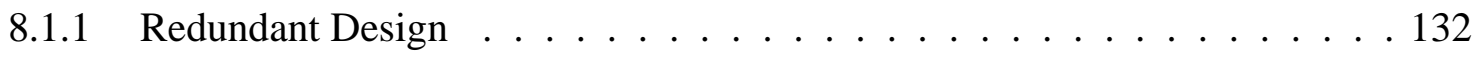

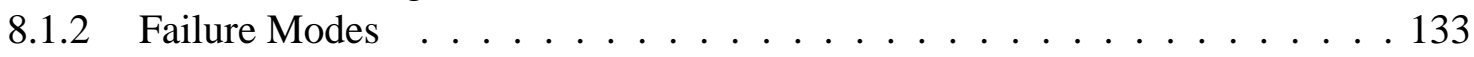

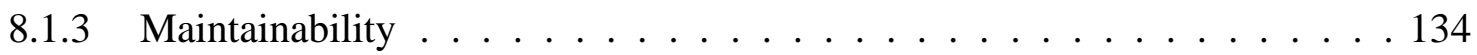

8.1.4 Power Supply Banks and Quench Rate . . . . . . . . . . . . . . . . . . . . . . . . . . . . . . . . . . . .

8.2 Reliability Models . . . . . . . . . . . . . . . . 135

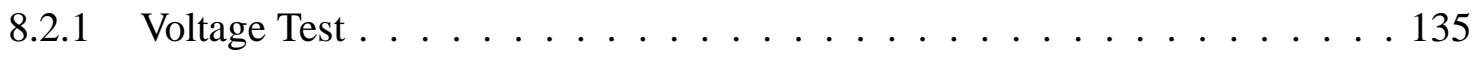

8.2 .2 Discharge Test . . . . . . . . . . . . . . . . . . . . . . . . . . . . . . . . . . . . . . . . .

8.3 Reliability Results . . . . . . . . . . . . . . . . . . . . . 140

8.4 Sensitivity Analysis . . . . . . . . . . . . . . . . . . . . . . . . . . . . . . . . . . . . . . . . . . . . .

8.5 DQHDS Maintenance Conclusions . . . . . . . . . . . . . . . . 144

9 Reliability of the LHC Energy Extraction Systems 145

9.1 Energy Extraction Design and Operation . . . . . . . . . . . . . . . . . . . 145

9.1.1 13 kA Energy Extraction . . . . . . . . . . . . . . . . . . . . . . . . . . . . . . . . . . . . .

9.1 .2600 A Energy Extraction . . . . . . . . . . . . . . 147 
9.1 .3 Operation and Reliability . . . . . . . . . . . . . . . . 147

9.1 .4 High Current Controllable Fuses . . . . . . . . . . . . . . . . . . . 148

9.2 Reliability Model . . . . . . . . . . . . . . . . . . . . . . 149

9.3 Reliability of the $13 \mathrm{kA}$ Energy Extraction Systems . . . . . . . . . . . . 151

9.3.1 Failure Modes and Maintainability . . . . . . . . . . . 151

9.3 .2 Input Parameters . . . . . . . . . . . . . . . . . . 151

9.3.3 Reliability Results . . . . . . . . . . . . . . . . 152

9.4 Reliability of the 600 A Energy Extraction Systems . . . . . . . . . . . . . . 155

9.4 .1 Input Parameters . . . . . . . . . . . . . . . . . . . . . . . . . . . . . . . . . . . .

9.4 .2 Reliability Results ... . . . . . . . . . . . . . . . . . . . . . . . . . . . . . . . . . .

9.5 Conclusions about Maintenance . . . . . . . . . . . . . . . . . . 158

10 The QPS and LHC Machine Dependability 161

10.1 Machine Interlock Systems . . . . . . . . . . . . . . . . 161

10.2 Powering Interlock System . . . . . . . . . . . . . . . . . 162

10.3 Power Abort from QPS . . . . . . . . . . . . . . . . . . . . . . 164

10.3.1 Power Abort Signals Between QPS, Power Converters and PIC . . . . . . 164

10.3.2 Power Abort Reliability . . . . . . . . . . . . . . . . . . . . . . . . . . . . . . . . . . . . .

10.4 Power Permit and Maintainability . . . . . . . . . . . . . . . . . . . . . . . . . . . . . . . . . . . . . . . . .

10.5 RESQP During LHC Operation . . . . . . . . . . . . . . . . . 168

$\begin{array}{lr}\text { Conclusions and Outlook } & 169\end{array}$

A Radiation Test Results $\quad 171$

A.1 Voltage References . . . . . . . . . . . . . . . . . . . 171

A.2 Linear Voltage Regulators . . . . . . . . . . . . . . . . . . 172

A.3 Bipolar Dual Timers . . . . . . . . . . . . . . . . . . 172

A.4 Instrumentation Amplifiers . . . . . . . . . . . . . . . . . . . . . . . . . . . . . . . . . . . . . . . .

A.5 Switch Mode Power Supplies . . . . . . . . . . . . . . . . . . . . . . . . . . . . . . . . . . . . . . . . .

A.6 Data Acquisition . . . . . . . . . . . . . . . . . . . . . . . . . . . . . . . . . . . . . . . . . . . . . .

A.7 Quench Heater Power Supplies . . . . . . . . . . . . . . . . . . 174

$\begin{array}{llr}\text { B Reliability Data From Life Testing } & 177\end{array}$

$\begin{array}{llr}\text { C Safety Integrity Level } & 179\end{array}$

$\begin{array}{lr}\text { Bibliography } & 181\end{array}$

$\begin{array}{lr}\text { List of Figures } & 187\end{array}$

$\begin{array}{lr}\text { List of Tables } & 190\end{array}$

$\begin{array}{lr}\text { Acknowledgements } & 192\end{array}$ 


\section{Introduction}

The LHC (Large Hadron Collider) is the next circular accelerator being constructed at CERN. It will provide head-on collisions of protons at a centre of mass energy of $14 \mathrm{TeV}$ for high energy particle physics research. The LHC is currently being installed in the $27 \mathrm{~km}$ long LEP tunnel. In order to reach the required magnetic field strengths, superconducting magnets cooled with superfluid helium will be installed.

The energy stored in the superconducting magnets is very high $(10 \mathrm{GJ}$ in the electrical circuits and $700 \mathrm{MJ}$ in the circulating beams) and can potentially cause severe damage when the superconducting state disappears (quench) due to beam losses or cryogenic failures. The very flexible LHC optics requires about 10,000 magnets connected with 1,700 electrical circuits. All superconducting elements require protection in case of a quench or other hazards which may perturb the accelerator operation or even damage the equipment. Some elements require complex design, and for others the protection can be fairly simple depending on the design of the elements and their powering. This thesis has been developed within the CERN team in charge of these issues.

Although other accelerator using superconducting technology have been built and work well (e.g. Tevatron, HERA, RHIC), this new collider is a much more complex machine, whose success will not be defined only by the two classical objectives in a high energy particle accelerator, energy and luminosity, but also by its reliability and availability performance.

Reliability theory for complex systems was developed in the early fifties, however it has not been applied to large scale test facilities until this century. Existing research accelerators are not optimised from the reliability point of view and all former studies show a lack of data and, above all, methodology.

This thesis focuses on applying and adapting the reliability theory and methods that are currently used by industry and small accelerators to the protection systems of larger and more complex machines like the LHC. In the framework of this thesis, two main contributions have been achieved. Firstly, an analytical model has been developed for a general multi-state protection system which is able to include the specific characteristics of a large superconducting accelerator. In order to provide a tool for its implementation and related computations, the simulation program RESQP (REliability Software for Quench Protection studies) has been developed. Secondly, we have carried out a detailed analysis of the LHC Quench Protection System reliability. The hardware design and the maintenance strategy during the machine operation have been optimised.

The LHC motivation, characteristics and main challenges are presented in Chapter 1 . The basics of superconductivity and the accelerator magnets built with this technology are introduced in Chapter 2. The chapter explains the quench process and the different strategies to protect the magnet after the transition. A short introduction to superfluidity and Helium is included at the end of the chapter.

Chapter 3 details the different superconducting systems of the machine: electrical circuits, busbars, HTS current leads and the cryogenic system. The chapter explains the quench protection 
strategy adopted for the different LHC superconducting components and describes the required hardware. The indispensability of a reliable protection system is introduced.

The concepts and methods used in reliability engineering are explained in Chapter 4 . The chapter presents the most used failure distributions, and the mathematical tools for modelling the reliability of complex systems. The Quench Protection System and, in general, the LHC reliability needs are explained together with the possible applications of methods and data used by brother fields such as the nuclear, military, and space industries.

Chapter 5 is devoted to explain the methodology that I propose in order to study and improve the reliability of an accelerator protection system from the design phase.

The next chapter explains the experiments and simulations carried out in order to validate the protection strategy. The LHC Test String 2 and the used simulation programs are presented. The chapter finishes with a broad analysis of the possible failure modes of the system.

The reliability of the main subsystems of the quench protection, the quench detectors, the quench heater power supplies and the energy extraction facility, are detailed in Chapters 6, 7 and 8 , respectively.

Finally, in Chapter 10, the global impact of the Quench Protection System on the LHC performance during the 20 years of LHC operation is predicted. In this chapter, I define a maintainability program proposal to reach the desired reliability level. The utility of RESQP during the machine operation as feedback of the design decisions is explained. 


\section{Chapter 1}

\section{The Large Hadron Collider}

\subsection{High Energy Particle Physics Today}

Particle physicists have found that they can describe the fundamental structure and behaviour of matter within a theoretical framework called the Standard Model. This model incorporates all the known particles and forces through which they interact, with the exception of gravity. It is currently the best description we have of the world of quarks and other particles. However, the Standard Model in its present form cannot be the whole story. There are still missing pieces and other challenges for future research to solve.

The masses of the particles vary within a wide range of masses. The photon, carrier of the electromagnetic force, and the gluons that carry the strong force, are completely massless, while the conveyors of the weak force, the $\mathrm{W}$ and $\mathrm{Z}$ particles, each weight as much as 80 to 90 protons or as much as reasonably sized nucleus. The most massive fundamental particle found so far is the top quark. It is twice as heavy as the $\mathrm{W}$ and $\mathrm{Z}$ particles, and weights about the same as a nucleus of gold. The electron, on the other hand, is approximately 350,000 times lighter than the top quark, and the neutrinos may even have no mass at all.

Why there is such a range of masses is one of the remaining puzzles of particle physics. Indeed, how particles get masses at all is not yet properly understood. In the simplest theories, all particles are massless which is clearly wrong, so something has to be introduced to give them their various weights. In the Standard Model, the particles acquire their masses through a mechanism named after the theorist Peter Higgs. According to the theory, all the matter particles and force carriers interact with another particle, known as the Higgs boson. It is the strength of this interaction that gives rise to what we call mass: the stronger the interaction, the greater the mass. If the theory is correct, the Higgs boson must appear below $1 \mathrm{TeV}$. Experiments at Tevatron and LEP have not found anything below $110 \mathrm{GeV}$.

Another open question is the unification of the electroweak and strong forces at very high energies. Experimental data from different laboratories around the globe confirm that within the Standard Model this unification is excluded [1]. When scaling the energy dependent constants of the electroweak $\left(\alpha_{1}\right.$ and $\left.\alpha_{2}\right)$ and strong $\left(\alpha_{3}\right)$ interactions to very high energies, the coupling constants do not unify. Grand Unified Theories (GUT) explain the Standard Model as a low energy approximation. At energies in the order of $10^{16} \mathrm{GeV}$, the electromagnetic, weak and strong forces unify. One of the GUT theories is the supersymmetry (SUSY) that predicts new particles to be found in the TeV range. Many other GUT theories predict new physics at this energy scale.

These and other questions like the elementarity of quarks and leptons, the search of new quark families and gauge bosons or the origin of matter-antimatter asymmetry in the Universe, will 
be addressed by CERN's next accelerator, the Large Hadron Collider, which is currently under construction.

\subsection{The LHC Project}

The Large Hadron Collider [2] will collide two counter-rotating proton beams at a centre of mass energy of $14 \mathrm{TeV}$. This energy is seven times higher than the beam energy of any other proton accelerator to date. In order to achieve an unprecedent luminosity of $10^{34} \mathrm{~cm}^{-1} \mathrm{~s}^{-2}$, it must operate with more than 2800 bunches per beam and a very high intensity. The machine can also be filled by lead ions up to $5.5 \mathrm{TeV} /$ nucleon and therefore allow heavy-ion experiments at energies about thirty times higher than at the Relativistic Heavy Ion Collider (RHIC) at the Brookhaven National Laboratory in New York. A summary of the main machine parameters is given in Table 1.1

\begin{tabular}{|c|c|c|}
\hline Energy & $\mathrm{TeV}$ & 7 \\
Injection Energy & $\mathrm{TeV}$ & 0.45 \\
Dipole Field & Tesla & 8.36 \\
Number of dipole magnets & & 1232 \\
Number of quadrupole magnets & & 430 \\
Number of corrector magnets & & $\approx 8000$ \\
Luminosity & $\mathrm{cm}^{-2} \mathrm{~s}^{-1}$ & $10^{34}$ \\
Coil aperture in arcs & $\mathrm{mm}$ & 56 \\
Distance between apertures & $\mathrm{mm}$ & 194 \\
Particles per bunch & & $10^{11}$ \\
Number of Bunches & & 2835 \\
\hline
\end{tabular}

Table 1.1: Some machine parameters [3].

\subsubsection{LHC Motivation}

The primary task of the LHC is to make an initial exploration of the $1 \mathrm{TeV}$ range. The major LHC detectors, ATLAS (A Toroidal LHC AparatuS) and CMS (Compact Muon Solenoid) should be able to accomplish this for any Higgs mass in the expected range. To get into the $1 \mathrm{TeV}$ scale the needed beam energy is $7 \mathrm{TeV}$.

Together with ATLAS and CMS, two other experiments will be fed by the LHC: a dedicated heavy ion detector, ALICE, which will be built to exploit the unique physics potential of nucleusnucleus interactions at LHC energies, and LHC-B, which will carry out precision measurements of $\mathrm{CP}$-violation and rare decays of $\mathrm{B}$ mesons.

The LHC has been prepared since the beginning of the eighties, with a $\mathrm{R}+\mathrm{D}$ program for superconducting dipole magnets and the first design of the machine parameters and lattice. The CERN Council approved the LHC in 1994. At that time it was proposed to build the machine in two energy stages due to limiting funding. Strong support for LHC from outside the CERN member states was found and CERN Council decided in 1996 to approve the LHC to be built in only one stage with $7 \mathrm{TeV}$ beam energy. Civil engineering works for the LHC are almost completed. The series production of the magnets has already started and works well. The prototypes String I and II have shown the feasibility of high magnetic field cryomagnets connected in series. Installation 
of the LHC components into the tunnel started after removal of LEP was completed. Injection into first octant is foreseen for 2006. It is planned to complete the machine installation and to start operation in 2007 [4].

\subsubsection{Technology Challenges}

\section{Superconducting Magnets}

In a circular accelerator of ions with mass $A$ and charge $Q$, the kinetic energy $K$ is given by the relation

$$
\frac{K}{A}\left(\frac{K}{A}+2 W_{0}\right) \approx\left(\frac{Q}{A}\right)^{2}(300 B R)^{2}
$$

where $W_{0}$ is the rest energy and $B$ the mean magnetic field. The energies are in $\mathrm{MeV}$, the magnetic field in Tesla and the radius in meter. For a high energy proton or electron $A=1, W_{0} \ll$ $K$ so $K=W$ and the equation 1.1 is reduced then to

$$
W \approx 300 B R
$$

The LHC is being installed in the 27-kilometre LEP tunnel, hence a 8.3 Tesla dipole field is needed in order to deflect the proton beams. This magnetic field can only be achieved at an acceptable cost using superconducting technology [3], by cooling magnets to $1.9 \mathrm{~K}$ with superfluid helium. The attainment of $7 \mathrm{TeV}$ in the existing tunnel presents some considerable technological challenges. The small tunnel cross section as well as the need for cost reduction imposes a twoin-one magnet design for the main dipoles and quadrupoles. The LHC machine is actually two accelerators sharing the same cryostat.

The very flexible LHC optics requires a large number of superconducting magnets, their connections with superconducting bus bars and current leads as part of the electrical circuits. In total, about 10,000 magnets connected within 1,700 electrical circuits will be installed.

\section{Stored Energy and Quenches}

The energy stored in the superconducting magnets is very high (10 GJ in the electrical circuits and $700 \mathrm{MJ}$ in the circulating beams [5]), and can potentially cause severe damages when the superconducting state disappears due to beam losses or cryogenic failures. The resistive transition from the superconducting to the normal-conducting state is called a quench. When it occurs, unless precautions are taken, the stored magnetic energy can generate excessive voltages and overheating whose consequences may lead to magnet degradation, a short circuit due to a melted insulation or even an open circuit, which occurs when the conductor burns out.

A reliable active Quench Protection System (QPS) is needed to bring the current down to zero safely when a quench occurs in order to assure the integrity of all the superconducting elements in the machine. 


\section{Cryogenics}

Cooling more than $31,000 \mathrm{Tm}$ of material spread over $27 \mathrm{~km}$ to below $2 \mathrm{~K}$ presents a considerable technological challenge. The most convenient way to cool helium to this temperature is to reduce the vapour pressure above the liquid bath. When the pressure is reduced in the heat exchanger below 20 mbar the helium, already in a superfluid state, reaches the $1.9 \mathrm{~K}$ operating temperature. The bulk of the cold masses remains at atmospheric pressure and are cooled down by the effect of exchange of heat. The machine will be cooled by eight cryoplants, each with an equivalent capacity of $18 \mathrm{~kW}$ at $4.5 \mathrm{~K}$. Four of these will be the existing LEP refrigerators upgraded in capacity from $12 \mathrm{~kW}$ to $18 \mathrm{~kW}$ and adapted for LHC duty. The other four new plants, unlike those of LEP, will be entirely installed on the surface, reducing the need of additional underground infrastructure.

The machine cryostats are fed from the cryogenic distribution line (QRL) that runs parallel to the superconducting magnets. The magnets of the arcs and the dispersion suppressors of one octant (see section 1.3) are housed in a common cryostat of diameter $914 \mathrm{~mm}$, which is about $3 \mathrm{~km}$ long with a cold mass of more than 5,000 Tm. The cooldown of the cold mass takes around 14 days.

When a magnet quenches, the generated heat is transferred to the helium bath and increases the helium pressure. The LHC cryostats are equipped with quench relief valves to release the pressure and to recover the helium by a high volume vacuum-insulated recovery line.

The high intensity beams in the LHC will deposit heat into the cryogenic surface surrounding the beam through a number of effects. The most important of these are the image currents (up to about $0.8 \mathrm{~W} / \mathrm{m})$ and synchrotron radiation $(0.6 \mathrm{~W} / \mathrm{m})$. These heat loads cannot be taken at $1.9 \mathrm{~K}$ and will be intercepted by a beam screen fitted inside the magnet cold bore and cooled by circulation of non superfluid helium between $5 \mathrm{~K}$ and $20 \mathrm{~K}$. As a result, the LHC requires a mix of refrigeration duties at several temperature levels.

The whole cryogenic system has 40,000 joints between pipes that have to be specially designed and mounted since the superfluid nature of the coolant would generate leaks at any microscopic hole.

The first phase of the cool down will be carried out with nitrogen due to its operation simplicity and, above all, its lower cost. It is estimated that about 12 million litres will be evaporated during that phase. Afterwards, 700,000 litres of helium will be necessary to set the whole machine at $1.9 \mathrm{~K}$. The LHC will be the biggest concentration of superfluid liquid in the Univers.

\section{Vacuum Systems}

Several types of vacuum enclosures are required by the LHC: the insulation vacuum in the cryostat to reduce heat conduction and convection and the beam vacuum to reduce the number of collisions of the beams with residual gas molecules.

Insulation vacuum systems are used for the magnet cryostat and the cryogenic distribution line. They reduce the heat transfer from the environment at room temperature to the cold parts due to conduction and convection. The working conditions are at a pressure of $10^{-4} \mathrm{~Pa}$ to $10^{-6} \mathrm{~Pa}$ in the temperature operating range from $1.9 \mathrm{~K}$ to $293 \mathrm{~K}$.

The vacuum chamber is placed in the bore of the magnet at an operating temperature of $1.9 \mathrm{~K}$. The beam screen described above provides a beam vacuum of $10^{-11} \mathrm{~Pa}$. Nevertheless, the energy associated to the inelastic scattering of protons with the residual gas cannot be intercepted by this screen and must be transported away by the superfluid helium. These collisions not only have an impact on the coil heat load but also on the radiation environment outside the cryomass due to the hadronic cascades generated by the scattered protons. If the vacuum chamber is not high enough the integrity of the electronic devices in the tunnel can be compromised. 
Recently an additional heat source has been identified, both secondary and photo- electrons accelerated across the beam pipe due to the bunched nature of the beam. Under unfavourable conditions, this could result in a resonant build-up of the electron cloud (multipactor) heavily loading the cryogenic system and causing beam instability. The solution to this issue is currently under investigation.

\section{Radiofrequency}

The particles are injected in the LHC from the last pre-accelerator, the SPS. The RF frequency of the LHC (44.8 MHz) is then the highest multiple of the SPS RF frequency (200.4 MHz) compatible with the length of the SPS bunches at transfer. Eight single cavities per beam are needed. The maximum operating voltage per cavity is $2 \mathrm{MV}$, which corresponds to a very conservative average accelerating gradient of $5 \mathrm{MV} / \mathrm{m}$. The cavities are made of copper with a thin film of niobium sputtered on the inside surface, identical to those used in the second phase of the LEP accelerator.

\section{Reliability and Availability}

Although other superconducting accelerators have already been successfully working for rather long time (e.g. mainly Tevatron and HERA and, more recently, RHIC), this new collider is a much more complex machine, whose success will not be defined only by the two classical objectives of a high energy particle accelerator, energy and luminosity, but also by its reliability and availability performance. Together with these basics goals, there are aspects like the large energy stored in the magnets, the number of critical components and the long repair times, that give to the LHC reliability analysis a much more important role than it had for other accelerators.

Reliability theory for complex systems was developed in the early fifties, however it has not been applied to large scale test facilities until this century. Existing research accelerators are not optimised from the reliability point of view and all former studies show a lack of data and, above all, methodology.

Although there is experience in reliability engineering at CERN [6, 7], until nowadays reliability has not been used as a general tool for complex accelerator design. A proper approach to the LHC reliability must take profit of the broad technical experience of the institute together with data from other laboratories in order to create models to predict the system reliability or, in other words, quantify the lack of knowledge.

\subsection{LHC Layout}

The LHC has an 8-fold symmetry with eight arc sections and eight long straight sections. Two counter-rotating proton beams will circulate in separate beam pipes installed in the same magnet (twin-aperture). The beams will cross over at the four experiments resulting in an identical path length for each beam.

Each arc consist of 23 identical cells (see Fig. 1.1), giving the total length of $2465 \mathrm{~m}$. Cells are formed by six $15 \mathrm{~m}$-dipole magnets and two quadrupole magnets (these dipoles and quadrupoles are called lattice or main magnets). Dipole magnets are used to deflect the beam whereas quadrupole magnets act as lenses to focus the beam. Different from an optical lens, a magnetic lens focuses in one transverse direction and defocuses in the other one. In order to obtain a net focusing effect, two quadrupole magnets are needed (similar to the principle of Galileo's telescope). This is a $F O D O$-lattice, in which $F$ and $D$ stand for the focusing and defocusing quadrupole. In a circular 


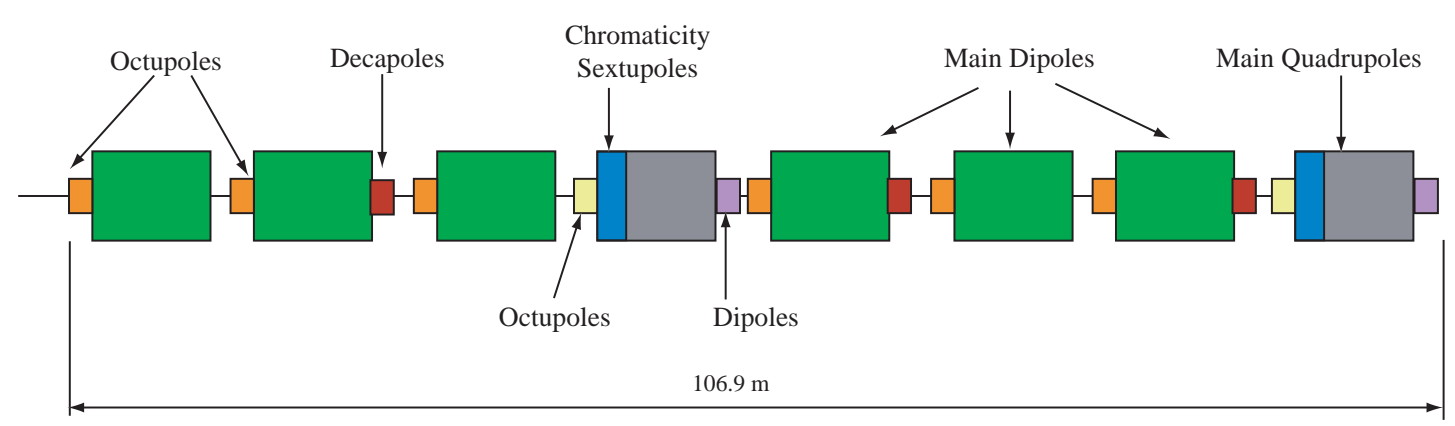

Figure 1.1: LHC cell layout: the six main dipole magnets, two lattice quadrupoles and correctors.

accelerator the $O$ stands for dipole used to bend the beam. Small dipole, sextupole, octupole and decapole corrector magnets are installed to keep the particles on stable trajectories.

The lattice quadrupole magnets and the corrector magnets of a particular half-cell form a socalled short straight section (SSS) and are housed in a common cold mass and cryostat.

At the beginning and the end of the straight sections a dispersion suppressor cell consisting of four quadrupoles interleaved with four strings of two dipoles each, is in charge of correcting the orbit deviation due to the drift in the energy of the particles.

The four long straight sections where the experiments are located, are formed by the dispersion suppressors and the insertion magnets. These last ones quick the separated beams to a common pipe where they are finally focused by the so-called inner triplet magnets in order to get very low- $\beta^{1}$ beams before collisions inside the detectors.

The other insertions (see Fig. 1.2) are to be used by systems for the machine operation: beam dump, beam cleaning (collimation), RF-cavities (accelerator units) and injection from preaccelerators.

The injector complex includes many accelerators at CERN: linacs, booster, LEAR as an ion accumulator, PS and the SPS. The beams will be injected into the LHC from the SPS at an energy of $450 \mathrm{GeV}$ and accelerated to $7 \mathrm{TeV}$ in about $30 \mathrm{~min}$, and then collide for many hours.

\footnotetext{
${ }^{1}$ The accelerator physics parameter $\beta$ refers to the betatron oscillation, which is related to the focalisation of the beam.
} 


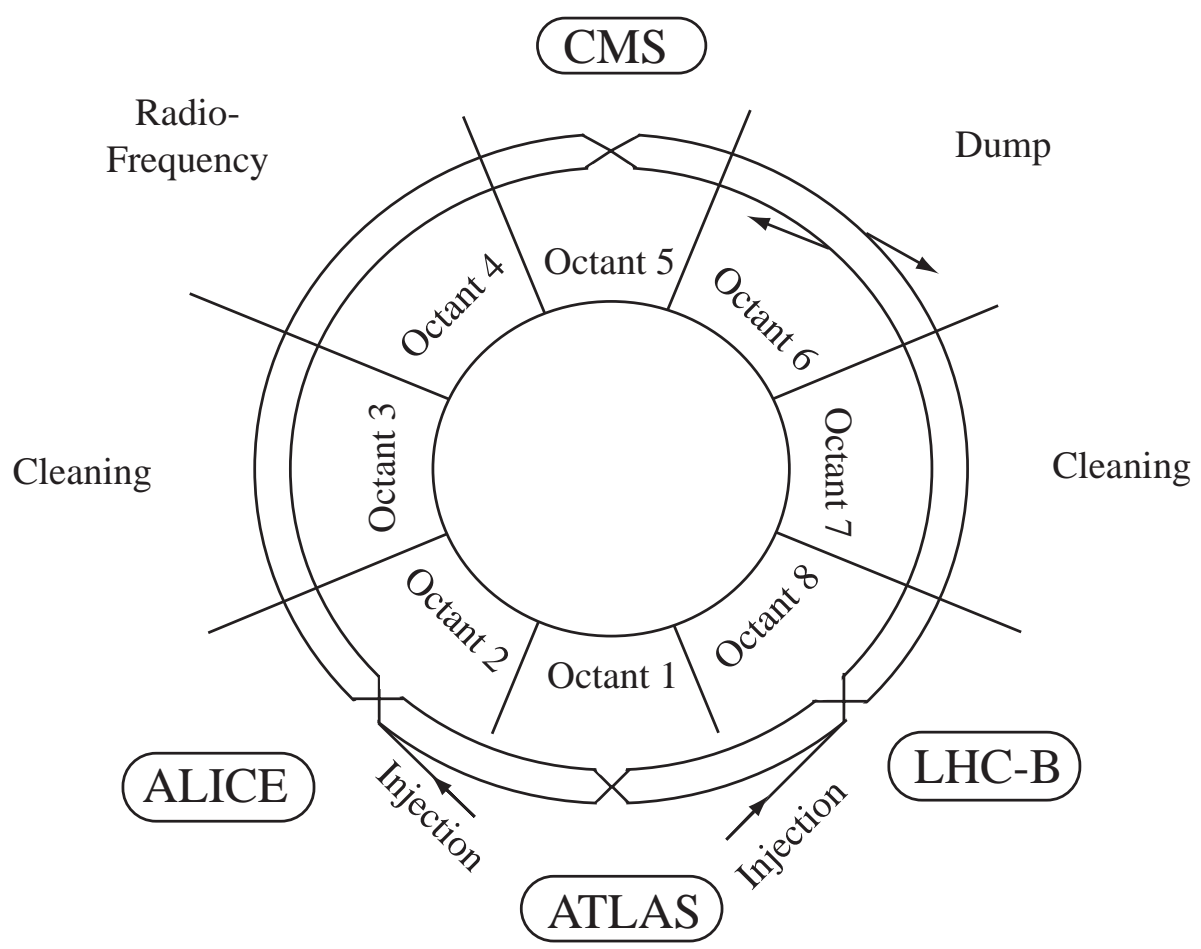

Figure 1.2: Layout of the Large Hadron Collider. 



\section{Chapter 2}

\section{Superconductivity and Superconducting Magnets}

\subsection{Basics of Superconductivity}

Superconductivity is a remarkable phenomenon whereby certain materials, when cooled to very low temperatures, become perfect conductors of electricity. From experiments [8] we know today that the resistivity in the superconducting state is at least $10^{12}$ times smaller than the resistivity in the normal-conducting state.

There exist two reasons for the development of superconducting technologies within accelerator projects: superconducting magnets allow higher particle energies for a given accelerator size, promising a substantial saving in the operating cost of the machine. Normal magnets with iron pole shoes are limited to dipole fields of about 2 Tesla and quadrupole gradients of $10 \mathrm{Tesla} / \mathrm{m}$ whereas with superconducting coil fields of more than 8 Tesla and gradients in excess of $200 \mathrm{Tesla} / \mathrm{m}$ are safely accessible.

\subsubsection{History Overview}

Superconductivity was discovered by H. Kamerlingh Onnes [9] in 1908 while he was studying the conductivity of metals at very low temperatures. He found that the resistance of mercury dropped to an unmeasurable small value just at the boiling temperature of liquid helium, which he had succeeded in liquefying just three years before. Kamerlingh Onnes called this new phenomenon superconductivity and the temperature at which the transition took place was named the critical temperature $T_{c}$. Superconducting properties have been found in a large variety of metals and ceramics but not in the best conductors at room temperature like copper or gold, unless they are under very high pressure.

W. Meissner and R. Ochsenfeld [10] discovered in 1933 that a superconductor expels a weak magnetic field when cooled below $T_{c}$ while strong fields can break down superconductivity. This effect could not be explained by the Maxwell equations since it was a non-classical phenomena only explainable by the quantum theory.

H. and F. London developed a phenomenological explanation of the Meissner-Ochsenfeld effect in 1935 which was confirmed by the theoretical description of Bardeen, Cooper and Schrieffer in 1957, and is called the BCS theory [11]. The BCS theory explains superconductivity as a quantum-mechanical phenomena on a macroscopic scale and it is based on the assumption that the current below $T_{c}$ is not carried by simple electrons but rather by pairs of electrons of opposite 
momenta and spins, the so-called Cooper pairs. Since Fermi principle only affects to particles with fractional spin and not to those with zero or integer spins, all the Copper pairs (spin zero) can occupy a single quantum state, the BCS ground state, whose energy is separated from the single-electron states by an energy gap which in turn can be related to the critical temperature.

A discovery of enormous practical consequences was the finding that there exist two types of superconductors with rather different response to magnetic fields. Superconductors made of pure elements like lead or mercury where called type I superconductors and alloys like niobium-titanium or niobium-tin were named type II superconductors. This last class have two critical fields and temperatures. The Ginzburg-Landau theory, formed in 1950 [12], provides a phenomenological explanation for the distinction of the two types and can be described as a limiting case of the BCS theory.

The development of hard superconductors mainly made of the type II superconductor niobiumtitanium allowed the construction of magnets with strong fields (above 1 Tesla) for applications in research, medicine, particle detectors and accelerators.

In 1986 Bednorz and Muller [13] discovered superconductivity in ceramic copper oxides at much higher temperatures with respect to type I and II superconductors. Some of them can operate in boiling liquid nitrogen. Although a large effort has been made in advancing the development of high temperature superconductors, hard type II still remains at present the leading material for the construction of large superconducting facilities.

\subsubsection{Meissner-Ochsenfeld Effect}

When a magnetic field is raised in a perfect conducting cylinder from zero to a finite value $B$, a surface current is induced according to Lenz's law for exactly cancelling the applied field in the interior. Since the resistance in a superconductor is zero, the current will flow with constant strength as long as the external field is kept constant keeping the bulk of the cylinder field-free. The superconductor acts as a perfect diamagnetic material below the critical temperature $T_{c}$. When the external field rises while the temperature is higher than $T_{c}$, eddy currents are induced, which decay quickly because of the resistance, and the applied magnetic field penetrates the interior of the cylinder. If the cylinder is cooled down, at the very instant the temperature drops below $T_{c}$, a surface current is spontaneously created and the magnetic field is expelled from the interior of the cylinder. This phenomena is called the Meissner-Ochsenfeld effect and law of induction cannot explain it because the magnetic field is kept constant.

But superconductivity is more than just vanishing resistance. The transition between normalconducting and superconducting phases is comparable to different thermodynamic phases. London equations demonstrate that magnetic field does not stop abruptly at the superconductor surface but penetrates into the material with exponential attenuation. The bulk of the superconductor is then field free, but a magnetic field can be tolerated in a thin surface layer. The current flow will be, therefore, restricted to the same thin layer. The dimension of this conducting surface is determined by a very important parameter resulting from London equations [14], the London penetration depth

$$
\lambda_{L}=\sqrt{\frac{m}{4 \mu_{0} n_{s} e^{2}}}
$$

where $m$ and $n_{s}$ are the mass and density of the Cooper pairs respectively. For type I superconductors the penetration depth is quite small, 20 to $50 \mathrm{~nm}$, this is a first indication that type I superconductors are not suitable for winding superconducting magnet coils. 


\subsubsection{The Critical Magnetic Field}

The transition from normal-conducting to superconducting state can be described as a thermodynamic phase transition quantified by the free energy

$$
F=U-T S
$$

where $F$ is the free energy, $U$ the internal energy and $S$ the entropy. Below the critical temperature, the superconducting state has lower entropy than the normal state since paired electrons are collected in a single quantum state. Although the entropy difference between states is small (only a small fraction of the valence electrons are condensed into Cooper pairs) it is enough for provoking the phase transition.

If a magnetic field is applied to a normal conductor, its free energy will not change since the field will just penetrate it. On the other hand, a superconducting cylinder will be affected by the field setting up shielding currents that will increase its free energy. If the applied magnetic field is increased sufficiently, the free energy associated to the superconducting state will reach the associated to the normal state and the superconductivity will be lost. The magnetic strength at which the free energy in the superconducting state just equals the free energy in the normal state is called the critical magnetic field $B_{c}$ and it depends on the temperature.

\subsubsection{Type II Superconductors}

Type I superconductors do not allow the construction of superconducting magnets since the critical magnetic field is very small. The magnetisation curves of transition metals like niobium and some alloys show two critical fields $B_{c 1}$ and $B_{c 2}$ which are both temperature dependent. These materials are called type II superconductors. Below the critical field $B_{c 1}$ the material is in the Meissner phase with complete exclusion of the field from the interior like a type I superconductor. Between $B_{c 1}$ and $B_{c 2}$ the Meissner-Ochsenfeld effect is said to be incomplete (mixed phase) and the material keeps superconducting electrical properties up to this last value.

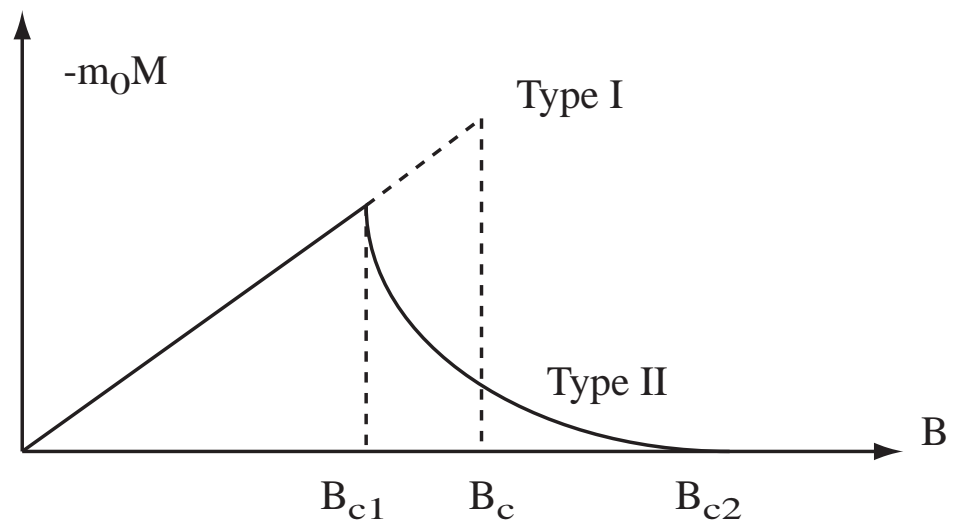

Figure 2.1: Magnetisation of a type II superconductor as a function of the applied magnetic field.

The upper critical field $B_{c 2}$ can assume very large values which make these substances very interesting for magnet coils. The magnetic flux does not penetrate the type II superconductor with uniform density, it is concentrated in flux tubes surrounded by a super-vortex current. The 
Cooper pair density drops to zero at the centre of the vortex, meaning that the core of a flux tube is normal-conducting.

\subsubsection{Hard Superconductors}

The large upper critical field of type II superconductors permits to flow high currents in the bulk material that, in principle, would allow the construction of accelerator magnets with them. However, there is a problem with the so-called flux flow resistance. The current density exerts a Lorentz force on the flux lines which begin to move in a direction perpendicular to the current and to the field. This is a viscous motion that leads to heat generation. So although the current itself flows without dissipation, the material acts as if it had an ohmic resistance.

In order to make coil wires this motion has to be inhibited. This can be done by capturing them at pinning centres. A type II superconductor is called a hard superconductors when it presents a strong pinning. Hard superconductors are very well suited for high field magnets as they allow current to flow in high magnetic fields without heat generation. The penalty of using hard superconductors is the strong magnetic hysteresis that generates persistent current multipoles.

Moreover, some small full creep effects remain. At finite temperatures a few of the flux quanta may be released from their pinning locations by thermal energy and move out of the material, reducing the magnetisation. This can be explained assuming a logarithmic decay of the critical current density $J_{c}$ with time. This implies that for a given temperature and magnetic field the critical current density depends on time. The value $J_{c}$ can be set after the decay rate has become unmeasurable small. Hard superconductors can be, therefore, characterised by a critical current density as a function of the applied magnetic field and temperature (see Fig. 2.2).

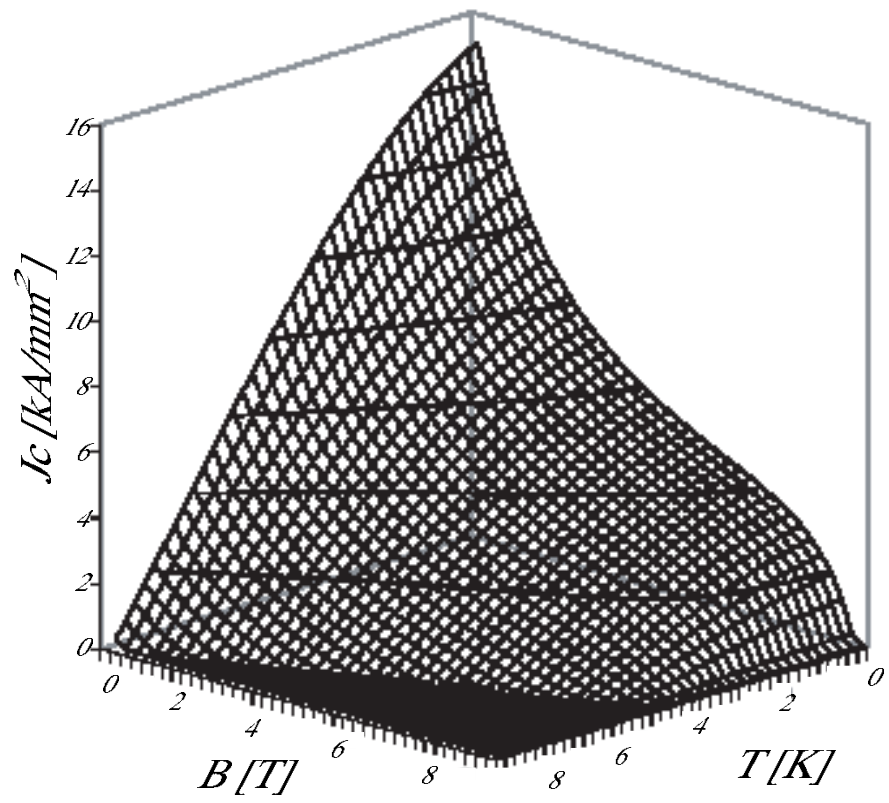

Figure 2.2: Critical surface of the NbTi superconductor used in the LHC.

Early attempts to use hard superconductors for shielding or trapping of magnetic fields faced 
the difficulty that under certain conditions the supercurrents suddenly broke down. In hard superconductors exposed to a high external magnetic field, a small heat dissipation makes the temperature rise, thus reducing the critical current density. This increases the core field resulting in a voltage perpendicular to the current flow and the applied magnetic field. The slightest disturbance will cause the superconductor to reduce its critical current and expel part of the captured magnetic flux. This process is called flux jumping. The stability increases as the conductor length along the voltage direction decreases [15]. In order to prevent flux jumping, superconductors that are used to wind magnets are made of multifilament wires.

\subsection{Superconducting Accelerator Magnets}

Superconducting magnets have been manufactured on a commercial basis for about 30 years and can now be regarded as a mature, but still evolving, industrial product. Accelerators such as the Tevatron, HERA and RHIC have so far constituted the largest individuals units of consumption. However, they are not the largest market since quite some time. Over the last decade, the largest yearly total sales have been the magnets for medical magnetic resonance imaging (MRI). In this section we will focus on superconducting magnets for high energy accelerators.

\subsubsection{Superconducting Materials for Accelerators}

A large variety of metals and alloys are superconductors at liquid helium temperature, but basically only niobium-titanium $\mathrm{NbTi}$ and niobium-tin $\mathrm{Nb}_{3} \mathrm{Sn}$ are commercially available for large scale magnet production.

\section{Niobium Titanium}

Niobium and titanium are mutually soluble to form ductile alloys over a wide range of compositions which must be properly chosen to get the desired critical properties. The ductility permits effective and simple fabrication of filament wires.

The upper critical magnetic field for $\mathrm{NbTi}$ alloys at $4.2 \mathrm{~K}$ for a reasonable current density is only about 8 Tesla, but when superfluid helium is used as coolant at $1.9 \mathrm{~K}$ this critical magnetic field increases to about 10 Tesla. This was the solution chosen for the LHC superconducting magnets.

\section{Niobium Tin}

Unlike $\mathrm{NbTi}, \mathrm{Nb}_{3} \mathrm{Sn}$ is a brittle intermetallic compound having a well defined stoichiometry. Because it is brittle, it cannot be fabricated by a wire-drawing process and must be formed in situ at its final size. $\mathrm{Nb}_{3} \mathrm{Sn}$ strands have to be reacted at $650^{\circ} \mathrm{C}$ to achieve full performance. In the fully reacted state multifilamentary $\mathrm{Nb}_{3} \mathrm{Sn}$ strands are quite sensitive to mechanical stress. The advantage of this alloy is a much higher critical field of about 20 Tesla.

\section{High Temperature Superconductors}

The discovery of high $T_{c}$ superconductors (HTS) in ceramic copper oxides such as $Y \mathrm{Ba}_{2} \mathrm{Cu}_{3} \mathrm{O}_{7-x}$, $\mathrm{Bi}_{2} \mathrm{Sr}_{2} \mathrm{Ca}_{2} \mathrm{Cu}_{3} \mathrm{O}_{x}$, or $\mathrm{Bi}_{2} \mathrm{Sr}_{2} \mathrm{CaCu}_{2} \mathrm{O}_{x}$, all with critical temperatures around $100 \mathrm{~K}$, gives an opportunity to construct magnets operating at fields significantly higher than 10 Tesla. 
For temperatures below $30 \mathrm{~K}$ these superconductors can carry current densities at a magnetic field strength that are unaccessible with metal alloys. At higher temperatures, for instance at the nitrogen liquefaction temperature of $77 \mathrm{~K}$, the use of HTS is restricted to low magnetic fields or lower current densities. In the LHC, HTS conductors are used in current leads that are the feedthroughs for the current from room temperature into the cold masses. The use of HTS permits the reduction of the heat load to the cryogenic system with respect to the conventional copper current leads [16].

\subsubsection{Stability of Superconducting Cables}

When the temperature exceeds $T_{c}$ at any point of a superconducting coil, a resistive transition takes place and the superconductor becomes normal conducting. If the cooling power is not high enough to recover the superconducting state, the resistive zone will spread out starting the irreversible phenomena called quench.

Superconducting cables for magnets have to be designed to avoid damage in case of a quench or, ultimately, to exclude the possibility of quench, which is known as cryo-stability. Since conductors like $N b T i$ have a high resistivity in the normal-conducting state, the superconductors are stabilised with copper. When the wire quenches the current redistributes and bypasses the superconductor by flowing through the copper. The copper also gives a higher stability of the superconducting state since it increases the enthalpy of the cable.

Figure 2.3 shows one of the LHC dipole strands where thousands of NbTi filaments with a diameter in the range of some $\mu m$ are twisted inside a copper matrix. The filaments are twisted to ensure equal current distribution and to minimise the strength of induced eddy currents.
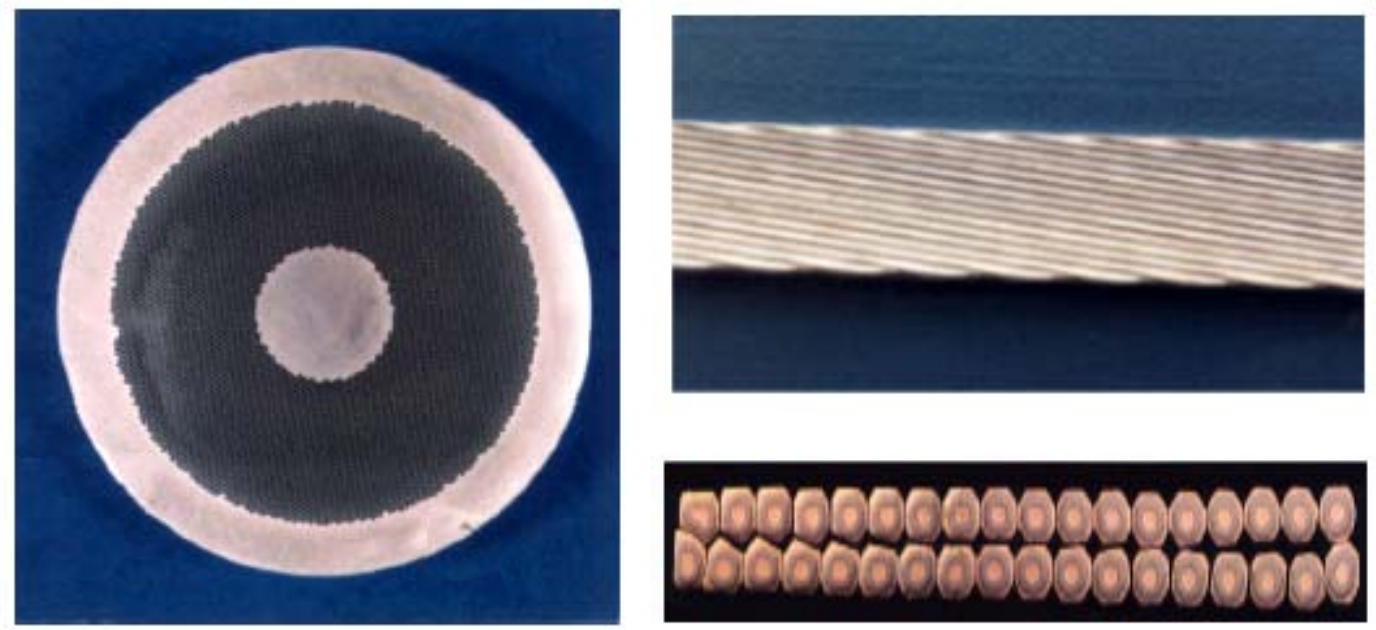

Figure 2.3: Cross section of the LHC main dipole strand (left) with $N b T i$ filaments (dark) embedded by a copper stabiliser (grey). The Rutherford cable (right) is used for the outer layer of the same magnets.

The strands in most superconducting magnets are twisted and compressed together into a flat, two-layer structure with a trapezoidal shape (see Fig. 2.3 right) forming what is called a Rutherford cable. This cable is permeable to liquid helium so that the surface of all the strands is wetted with the coolant. Due to its high heat capacity, the helium acts as a heat sink and stabilises the conductor in case of magnetic flux jumps or transient heat production caused by wire motion. In Tevatron 
and HERA the Rutherford cable is isolated with Kapton and glass-fibre epoxy, while RHIC and LHC coils have an all-Kapton insulation.

\subsection{Quenches and Quench Protection}

As it has been defined above, a quench is the transition from the superconducting to the normal state. If, due to some disturbance, part of a magnet coil is heated beyond the critical temperature the cable becomes normal-conducting in this region. Depending on the size of the zone, the cooling system may be sufficient to recover superconductivity or else the heating provoked by Joule dissipation is so violent that the transition is irreversible and then the magnet quenches.

It is a serious problem because superconductors are able to operate at such high levels of current density. In almost all superconducting elements (e.g. magnets, connections, current leads), an active protection system is needed in order to avoid overheating of the coil and inadmissible voltages across the circuits.

\subsubsection{Types of Quenches}

Basically one can distinguish two types of quenches: the natural quenches and the disturbance quenches. Training quenches usually occur during the first magnet powerings sometimes before the nominal field of the magnet is reached. This effect is called training, whereby the magnet shows a progressive improvement in performance after repeated quenching. It relates to small mechanical movements of the coil structure. After some training quenches, the magnets usually settle down to a fairly constant performance, quenching at currents which may be appreciably higher than the initial quench current. Hence, superconducting magnets are not memoryless systems but their performance depends on their magnetic and thermal history.

For the other kind of quench, the disturbance quench, the origin is usually a local overheating beyond the critical temperature. These quenches are mainly due to energy deposited into the coils by particles from the beam interaction with the residual gas of the beam pipe, failures in the cryogenic system or conductor motion. Since LHC magnets will be trained up to nominal field before being installed in the tunnel, the second kind of quenches will be most probably the only ones to be faced during normal operation.

\subsubsection{Coil Stability}

The stability of a coil is a measure of its ability to recover the superconducting state after a resistive transition in a small zone. The combination of two mechanisms can cause the recovery, the cooling due to helium and the heat conduction along the cable.

In the limiting case that only transverse cooling is present, recovery will take place if the cooling power is stronger than the ohmic heat generation. When the current of the composite wire $(N b T i-C u)$ enters in the resistive state, part of the current carried by the superconductor is transferred to the copper matrix (current sharing). The higher the copper to superconductor ratio, the smaller the ohmic generation will be. In order to have cryo-stable cables this ratio must be higher than 10, which would require very bulky and expensive coils. Therefore cryo-stability is not achievable at nominal operating current of the LHC. However, at lower currents the conductor may become cryo-stable since the heat generation increases with the square of the current. 
When the superconducting wires or cables have a poor contact to the helium coolant (e.g. epoxy-impregnated coils), the heat generated in a certain zone can only be removed by heat conduction along the wire. The resistive part expands if the generated heat exceeds the heat removed. The lower limit of the length that a resistive zone must have for developing a quench is the so called minimum propagation zone, which depends on the helium temperature and the wire heat conductivity. For pure $N b T i$ it is less than $1 \mu \mathrm{m}$ [17], but when the filaments are embedded in a matrix of high purity copper with electrical and thermal conductivities three order of magnitude better than those of normal conducting $N b T i$, the minimum propagation length becomes at least a factor of thousand larger (i.e. about $1.5 \mathrm{~cm}$ for the cable in the LHC main dipoles at nominal current [18]).

The energy to warm up the minimum propagation zone is called minimum quench energy and is usually in the range of 0.001-0.1 J. However, the real energy needed to start the quench is always larger that the minimum quench energy since the cooling of an accelerator magnet is based on both mechanisms, helium cooling and cable conduction.

The quenches during LHC operation will be mainly due to heat pulses where the adiabatic heating dominates. The helium contained in the voids of the Rutherford cable plays an important role, since it enhances the overall heat capacity of the cable by nearly two orders of magnitude [17].

\subsubsection{Quench Propagation}

The magnets in an accelerator are connected in series and when one magnet in the chain quenches it has to absorb not only its own stored energy but also the ones in series. It would be very undesirable that the quenched zone would not expand and only the temperature inside increased.

Regarding the adiabatic (no helium cooling) heat balance equation of a one-dimensional conductor:

$$
A \cdot c(T) \cdot \frac{L t}{d t}=\frac{d}{d x}\left(A(x) \cdot j_{x}\right)+G(T, t)
$$

where $A$ is the cross section of the conductor, $j_{x}$ the heat flux density along it and $G$ the ohmic heat generation:

$$
G(T, t)=\frac{\rho(T) I^{2}}{A}
$$

Since at a fixed current the heat generation term increases almost linearly with the temperature it is reasonable to assume that in the hottest part of the wire the temperature has to increase faster than in the coldest one. This is luckily not the case and the quench shows the opposite behaviour. The thermal properties of the materials at cryogenic temperature are rather different from the ones at room temperature because the heat capacity cannot be considered constant. The classical theory of matter cannot explain this because the reasons are rooted in the quantum mechanical properties. The heat capacity of any material goes to zero when the temperature goes to zero (see Fig. 2.4). In the wire then the highest temperature variations are in the regions of the front line between the superconducting and the normal state of $N b T i$ at around the critical temperature [19]. 
In any of the two zones, copper must have a good heat conductivity in order to allow heat propagation. An important technical parameter is the Residual Resistivity Ratio RRR, the ratio between the resistivity at room temperature and the one at $10 \mathrm{~K}$.

$$
R R R=\frac{\rho(300 K)}{\rho(10 K)}
$$

Manufacturers of superconducting wires usually specify a minimum guaranteed value for RRR ensuring a certain quality and purity of copper. In Fig. 2.4 copper resistivity is plotted for different RRR values.
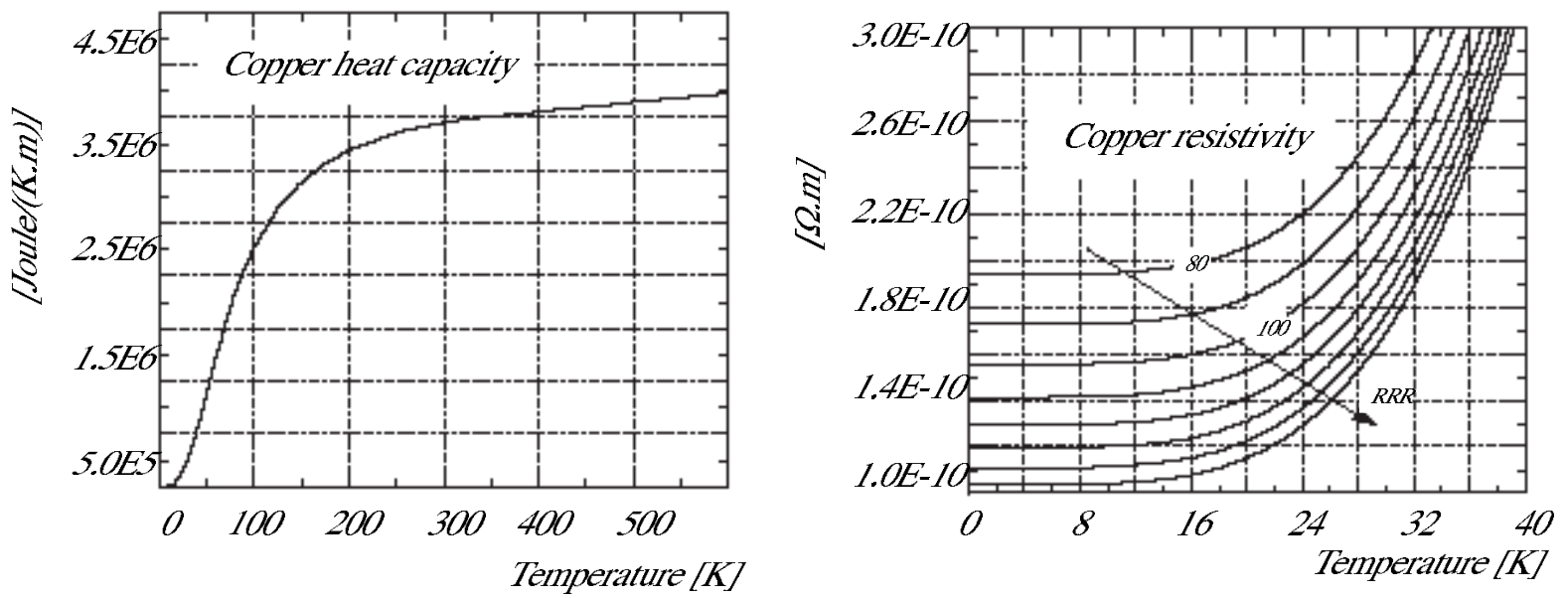

Figure 2.4: Copper heat capacity in function of the temperature (left) and copper resistivity for different RRR in function of the temperature (right).

Simulations and experiments carried out at CERN [18] have confirmed that the quench propagation velocity in the order of $10 \mathrm{~m} / \mathrm{s}$ in the main magnets at nominal current. This propagation is not fast enough for ensuring the integrity of the magnet after a quench, and an active protection system is needed to artificially quench the whole magnet after the resistive transition is detected.

\subsubsection{Protection of a string of magnets}

The stored energy in an LHC arc is in the order of $1 \mathrm{GJ}$ and, if no action is taken, it would be dissipated in the small quenched area. At the same time, due to the large inductance of the magnet chain, the current cannot be brought to zero instantaneously in the whole chain without generating a voltage to ground exceeding hundreds of kilovolts.

The quench protection system of an accelerator like the LHC is based on the following basis:

- Subdivision of the whole accelerator inductance, which is one of the reasons of the LHC sectorisation explained in Chapters 1 and 3.

- Fast quench detection.

- Isolation of the quenched magnet from the rest of the circuit. 
- Spreading of the energy stored in the quenched magnet.

- Controlled energy extraction of the whole magnet chain.

\section{Quench Detection}

The maximum temperature that appears in a magnet after a quench depends mainly on the decay time of the current. Typically time constants of much less than $1 \mathrm{~s}$ are needed to limit it to values below $300 \mathrm{~K}$. This requires a fast detection of the resistive transition in order to switch off the current in few hundred milliseconds. The detection is usually done by monitoring the resistive voltage created when a normal zone appears. The voltage as a function of time $V(t)$ across an entire magnet coil is

$$
V(t)=I(t) R(t)+L(I) \frac{d I(t)}{d t}-\sum_{i} M_{i} \frac{d I(t)}{d t}
$$

where $I(t)$ is the current, $R(t)$ the resistance, $L(I)$ the self inductance and $M_{i}$ the mutual inductance of a neighbouring turn or coil. The inductance depends on the current due to the influence of the iron lamination constituting the magnetic yoke of the magnet. However, for the LHC main magnets the iron yoke saturates for currents above $6 \mathrm{kA}$ and then the inductance can be considered constant. Corrector magnets are operated at a higher margin (smaller ratio of operating current to critical current) and their inductance is nearly independent of the applied current in the entire operating range.

Supposing the inductance is constant with current, the voltage developed in a quenching magnet can be expressed as

$$
V_{Q}=I(t) R(t)+L(t) \frac{d I(t)}{d t}
$$

When the accelerator is at collision energy the current in the magnets is constant and any voltage rising across the coil will be the sign of a resistive transition. A quench detector monitoring the total voltage across every magnet would be enough to detect any resistive transition. This is not the case, however, when the machine is not in an energy plateau (e.g. powering, acceleration, energy extraction, etc). The current is not constant with time hence an inductive voltage will appear. The quench detector must discriminate between the resistive part and the inductive part of the read voltage signal.

The quench detection system used in Tevatron, the first large superconducting accelerator, is based on the measurement of potential differences. Average values of the inductive voltages during ramps are calculated and used for comparison with the measured data. A significant discrepancy indicates a quench.

The system developed for HERA uses a bridge circuit for each dipole. The same idea is applied for the LHC main magnets where the bridge is made between the two symmetrical apertures of each dipole magnet and between two poles in the case of the quadrupoles. The inductive voltage in the two apertures (or poles) compensate each other perfectly and hence the bridge is very sensitive to small resistive voltages in the coil. The detection strategy for smaller magnets, busbars and current leads at the LHC is explained in Chapter 3. 


\section{Artificial Quench Propagation}

The energy stored in a magnet chain can be so enormous that the energy of the quenched magnet has to be spread along all the coil in order to avoid local overheating of the conductor that could lead to an open circuit.

This artificial quench is made via quench heater strips in close thermal contact with the coil. When a quench is detected, the energy stored in capacitor banks is released on the heaters warming up the whole magnet in a controlled way.

\section{Isolation of the Quenched Magnet}

In a chain of series-connected magnets, the quenching element will absorb the energy stored in the full chain. As indicated above, the stored energy in some of the LHC circuits is too large. To avoid dissipation of the full energy in one magnet, each one of them has a by-pass element.

The current has to be brought to zero with a long decay time ( $\tau=25 \mathrm{~s}$ for HERA and $\tau=110 \mathrm{~s}$ for LHC) to avoid excessive inductive voltages to ground. The current of the electrical circuit has to be guided around the quenched magnet to prevent destruction of the coil.

In Tevatron the discharge time is rather short (around 7 to $10 \mathrm{~s}$ ) and thyristors at room temperature act as fast switches. The decaying main current is bypassed through the thyristors. They are mounted outside the cryostats and hence current feedthroughs are needed.

In accelerators with long current decays like HERA or LHC the thyristors can be replaced with diodes mounted inside the cryostat. This scheme offers several advantages: there is not heat load in the cryogenic system from current leads, each magnet can be bypassed by its own diode and leaving out the current feedthrough makes the cryostat cheaper and easier to build. The cold diode model has been used in HERA and RHIC and it is also the choice for the LHC.

The energy at the still remaining superconducting magnets, once the quenching magnet is already properly isolated, has to be taken out from the rest of the circuit. Although, as it has been said above, this extraction cannot be too fast in order to avoid high voltages, it cannot be either too slow since the integrity of the bypassing diodes would be compromised. The current is commuted to properly dimensioned dump resistors by opening electro-mechanical circuit breakers.

In Fig. 2.5 an example of the actions taken after a magnet quenches in the LHC can be seen by following the voltage across the quenched magnet.

\section{Quench Load}

A very used term in quench protection in order to evaluate the strength suffered by a quenching magnet is the quench load. It is usually quantified by an unit called the MIIT, or $M \cdot A^{2} \cdot s$, calculated as follows:

$$
M I I T=\int_{T_{q}}^{\infty} I^{2}(t) d t
$$

Where $T_{q}$ is the quenching temperature. This value is useful for estimating the maximum temperature reached by a conductor after quench and therefore evaluating the effect of the quench protection system (see Chapter 6). 


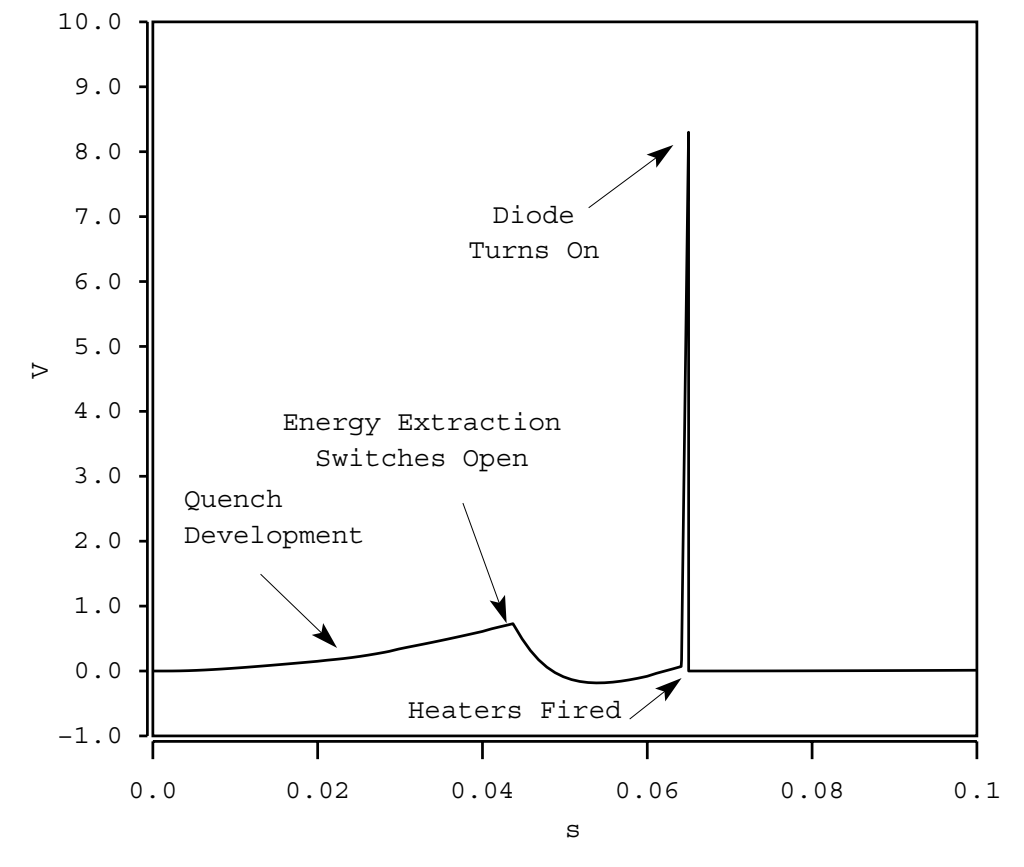

Figure 2.5: Voltage across an LHC main dipole after a quench.

\subsection{Superfluid Helium}

Superfluidity is a macroscopic quantum effect that can be explained in terms of the existence of a Bose condensate. There is a close analogy to superconductivity which relates to frictionless flow of electrons. Known superfluids are for example liquid ${ }^{4} \mathrm{He}$ below $2.17 \mathrm{~K}$ and liquid ${ }^{3} \mathrm{H}$ below $2 \mathrm{mK}$.

Superfluid helium flows without friction through a very narrow channel, provided that the flow velocity is less than a critical value, which depends on the channel width. This frictionless flow suggests vanishing viscosity, however the mechanical damping of a disc, which oscillates at a small amplitude around its own axis in superfluid helium, has been observed.

\section{Bose Condensation}

Unless it is under very high pressure, helium does not form a solid at $T=0$. Regardless of the state, the third law of thermodynamics requires that the entropy tends toward zero as the temperature reaches the absolute zero. Superfluid helium at $T=0$ must be then an ordered liquid that has experienced a disorder-order transition (He I to He II) at a certain temperature $T_{\lambda}$. This transition temperature would be analog to $T_{c}$ in superconductors.

Let us consider an ideal gas at very low temperatures. The atoms of this gas would be bosons (integer spin particles) obeying the Bose-Einstein statistics. This low temperature mechanics allows several bosons occupy the same quantum state, without violating the Pauli's exclusion principle of Fermi-Dirac statistics that only applies to particles with fractional spin (fermions) [20]. This is what is called Bose condensation of an ideal Bose gas. Below a certain critical temperature, a finite fraction of the bosons occupy the lowest quantum state in the vessel containing the gas, this 
fraction reaching unity at the limit $T \rightarrow 0$. All the particles are placed in the same quantum state, thus entropy vanishes.

However, all real gases liquefy at low temperatures. The Bose condensation actually occurs in some liquids at very low temperatures, but differs from the ideal Bose gas, since the fraction of atoms in the lowest momentum state does not reach unity but only about 0.1 at $T=0$.

Superfluid helium can therefore be considered as two-interpenetrating components: a normal component that disappears as temperature reaches the absolute zero, and a superfluid component that appears when the temperature drops below $T_{\lambda}$. This explains the damping of the oscillating disc.

\section{Superfluid Helium as Coolant}

Let us consider a tube of He II heated at one end and cooled at the other. Under these conditions, the superfluid component will move toward the heated end where, upon being heated, will be converted back to the superfluid component and carry the heat to the cooled end. As it cools it will be converted back to the superfluid component and move back to the warm end. Thus there is a counter flow of superfluid and normal fluid components between the heated and cool ends. This heat transfer mechanism is known as internal convection. It does not involve bulk mass transfer of the fluid but rather involves movement of the interpenetrating fluid components in the two fluid model [21].

The heat transfer properties of superfluid helium are based on this effect and strongly depend on the velocity of the superfluid. Below a certain critical velocity there is no interaction between the two components and the heat transfer at $1.9 \mathrm{~K}$ can be 1,000 times higher than for high purity copper. However, once this velocity is exceeded the superfluid part enters in a turbulent state interacting with the normal fluid part (mutual friction).

Nevertheless, heat transfer processes in helium are not dominated by internal convection but by the heat transferred from a heated surface to He II which is described by the Kapitza Conductance. This phenomenon, which is caused by the inefficiency of transferring energy between a solid and a fluid, is important at cryogenic temperatures, due to the good thermal properties of He II. This is theoretically explained in terms of phonon radiation. This conductance is the same in both static and flow.

In general superconducting magnets for accelerators such as the LHC are cooled in a helium bath, whereas other applications such as magnets for fusion reactors in a tokamak, they require cooling with a forced helium flow. Forced flow cooling increases the heat transfer by convection of helium which can then transport more heat away. 



\section{Chapter 3}

\section{LHC Superconducting Systems}

\subsection{LHC Main Magnets}

The LHC main magnets are the dipole magnets to deflect the beam and the quadrupole magnets in the arcs and the insertions around the experiments to focus it. The 154 main dipoles of an arc are powered in series. The arc (lattice) quadrupoles are powered by two independent circuits, one for the focusing and one for the defocusing apertures. In the insertions, the apertures of the quadrupoles are either powered individually or in series of two. Both, dipoles and quadrupoles are wound with Rutherford type (multistrand) cables insulated with polyimide films.

\subsubsection{Main Dipoles}

Among the 8,000 superconducting magnets required for the LHC, the most challenging are the 1,232 superconducting dipoles which must operate reliably at the nominal field of 8.3 Tesla, corresponding to the centre of mass energy of $14 \mathrm{TeV}$, with the possibility of being pushed to an ultimate field of 9 Tesla.

In the early days of magnet development, two technologies for the attainment of fields above 9 Tesla were investigated. The first of these involves the use of $N b_{3} S n$ at $4.2 \mathrm{~K}$. Indeed a dipole model with a first quench at 11 Tesla was built (Twente University) using this technology. However, the coils are very difficult and expensive to manufacture and they are not suitable for economic mass production. The other, more economical alternative is to use conventional NbTi technology at reduced temperature. This suffers from the drawback explained in Chapter 2 that the specific heat of the superconducting material and its associated copper matrix falls rapidly as the heat temperature is reduced. This makes the coil much more premature to quenches, due to small frictional movements of conductor strands or particle losses, since the adiabatic temperature rise is much higher at $1.9 \mathrm{~K}$ than at $4.2 \mathrm{~K}$. The special properties of superfluid helium can be used in part to compensate for this disadvantage.

The cross section of the twin-aperture LHC dipole magnet is shown in Fig. 3.1. The coil has a 6-block geometry design which optimised the field quality and allow more flexibility for small changes during series production.

The block geometry design is an approximation of the pure multipole field only achievable if the current distribution around the beam pipe is a function of the azimuthal angle $\phi$ given by

$$
I(\phi)=I_{0} \cos (m \phi)
$$




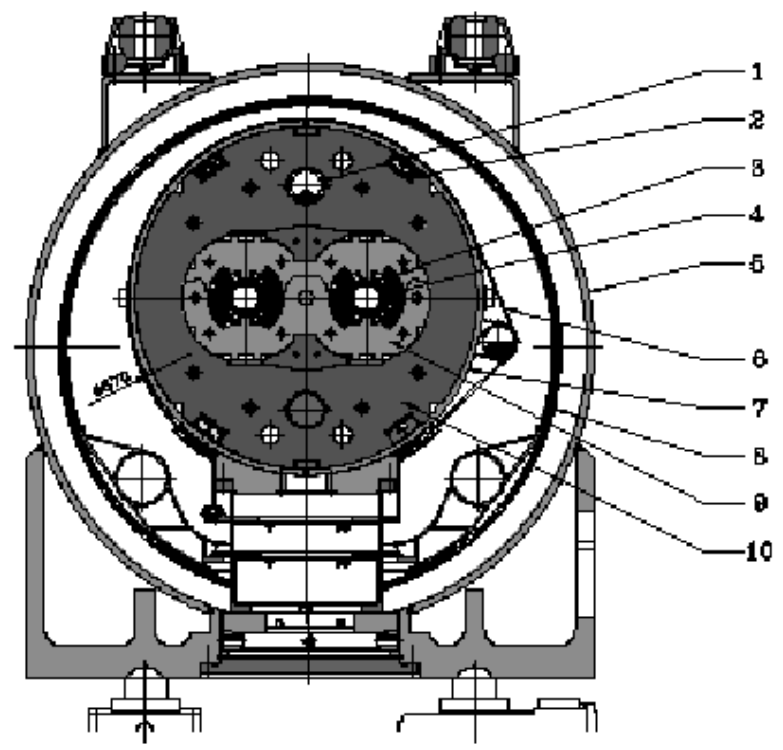

Figure 3.1: Twin-aperture LHC dipole magnet cross section, 1: heat exchanger pipe, 2: superconducting bus bars, 3: superconducting coil, 4: beam screen, 5: vacuum vessel, 6: radiation screen, 7: shrinking cylinder, 8: thermal shield, 9: non-magnetic collars, 10: iron yoke.

where $m$ is the order of the multipole ( $m=1$ in the case of the dipoles). Current distribution dependence are difficult to fabricate with a superconducting cable of constant cross section, hence they must be approximated by current shells or blocks [17].

The force containment structure consists in coil clamping elements: the steel collars, the iron yoke, the iron insert and the steel shrinking cylinder that contribute to keep the coils in their nominal position. The shrinking cylinder is also the outer shell of the helium tank. This cold mass is $15 \mathrm{~m}$ long and $23.8 \mathrm{Tm}$ heavy. It is bent to a $2,800 \mathrm{~m}$ radius of curvature in order to match the beam pipe (for more details see [22, 23]).

The parameters of the LHC main dipole magnets are summarised in Table 3.1, and the electrical connections sketched in Fig. 3.2.

Magnetic length

Operating temperature

Current at injection

Bending radius

Number of beams per magnet

Nominal current

Bending angle per magnet

Peak field in coil

Field at injection

Field at $7 \mathrm{TeV}$

Inductance per magnet

Mass and cold mass
$14.3 \mathrm{~m}$

$1.9 \mathrm{~K}$

$739 \mathrm{~A}$

$2803.928 \mathrm{~m}$

2

$11796 \mathrm{~A}$

$5.1 \mathrm{mrad}$

8.76 Tesla

0.53 Tesla

8.33 Tesla

$0.108 \mathrm{H}$

$23.8 \mathrm{Tm}$
Total length

Stored energy

$J_{N b T i}$ inner layer $7 \mathrm{TeV}$

$J_{N b T i}$ outer layer $7 \mathrm{TeV}$

Coil inner diameter

Coil outer diameter

Coil length

Number of blocks and layers

Number of turns inner layer

Number of turns outer layer

Cable length for inner layer

Cable length for outer layer
$15180 \mathrm{~mm}$

$7.1 \mathrm{MJ}$

$1200 \mathrm{~A} / \mathrm{mm}^{2}$

$1732 \mathrm{~A} / \mathrm{mm}^{2}$

$56 \mathrm{~mm}$

$120.5 \mathrm{~mm}$

$14567 \mathrm{~mm}$

6 and 2

$2 \times 15$

$2 \times 26$

$433 \mathrm{~m}$

$751 \mathrm{~m}$

Table 3.1: The main parameters of the LHC dipole magnets [18]. 


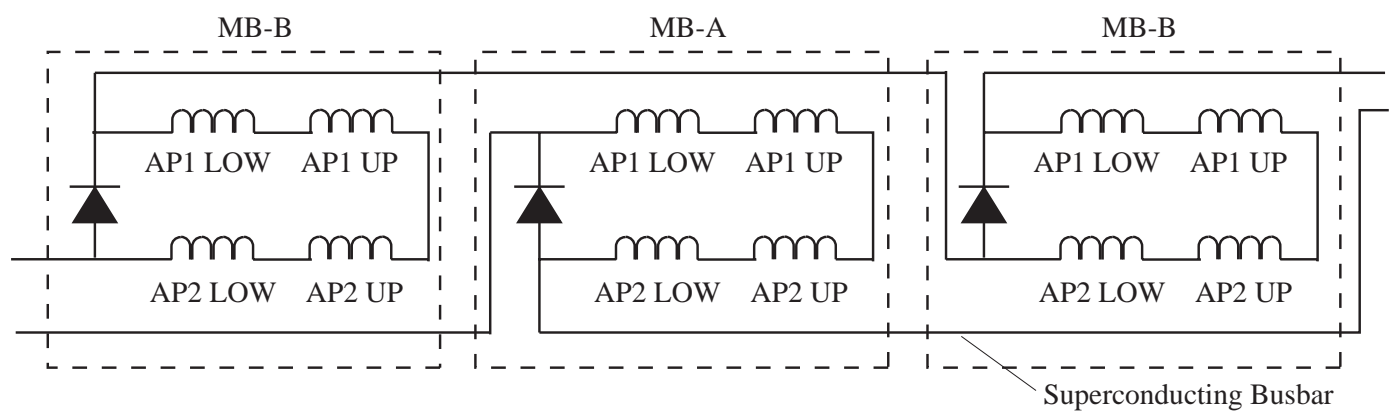

Figure 3.2: Electrical connection of the dipoles in one half cell. AP1 and AP 2 make reference to the two apertures (beam tubes) and LOW and UP to the upper and lower coil.

The main dipoles are classified into type A (MBA) and type $\mathrm{B}$ (MBB) depending on the electrical connections and the corrector magnets they host. They are alternately located along the arc in order to get a balanced-inductance circuit.

\subsubsection{Main Quadrupoles and Magnets in the Insertions}

The main quadrupole magnets for the LHC are the twin aperture or lattice quadrupoles (MQ) and the insertion quadrupoles (MQM family, MQY and MQX magnets).

The MQ quadrupoles provide the field to focus the particle beam and to keep it near the reference orbit. The LHC will feature 360, 3.25 m long lattice quadrupoles, designed for a $223 \mathrm{Tesla} / \mathrm{m}$ gradient field. The main parameters of these magnets are listed in Table 3.2.

Magnetic length

Peak field in coil

Gradient at injection

Nominal gradient

Geometrical aperture

Inductance per magnet

Stored energy

Max. rating current

Current density $7 \mathrm{TeV}$ (NbTi)

$3.10 \mathrm{~m}$
$6.86 \mathrm{Tesla}$
$14.3 \mathrm{Tesla} / \mathrm{m}$
$223 \mathrm{Tesla} / \mathrm{m}$
$56 \mathrm{~mm}$
$0.0112 \mathrm{H}$
$0.784 \mathrm{MJ}$
$13 \mathrm{kA}$
$1789 \mathrm{~A} / \mathrm{mm}^{2}$

Nominal Current
Current at injection
Coil inner diameter
Coil outer diameter
Coil length
Number of coil layers
Number of turns per coil (pole)
Cable length per pole
Total cable length

$11,870 \mathrm{~A}$

$763 \mathrm{~A}$

$56 \mathrm{~mm}$

$118.6 \mathrm{~mm}$

$3,184 \mathrm{~mm}$

2

24

$160 \mathrm{~m}$

$1280 \mathrm{~m}$

Table 3.2: Main parameters of the MQ magnets [18].

Unlike the main dipoles, the two coils sharing the same cryomass on the quadrupoles are powered by independent circuits that shift aperture every magnet in such a way that when the beam is horizontally focused in one aperture it is vertically focused (hence horizontally defocused) in the neighbour aperture. Moreover, the current bypasses every second magnet in order to get a balanced circuit (See Fig. 3.3).

The main lattice quadrupoles are housed in the so-called Short Straight Sections (SSS), they also contain several other kind of magnets (namely octupole, dipole and sextupole correctors, tuning quadrupoles and skew quadrupoles), the protection diodes and the beam position monitors.

The other group of main quadrupole magnets are the ones used in the insertion for the experiments. A schematic layout of the magnetic system for the high luminosity experiments ATLAS and 


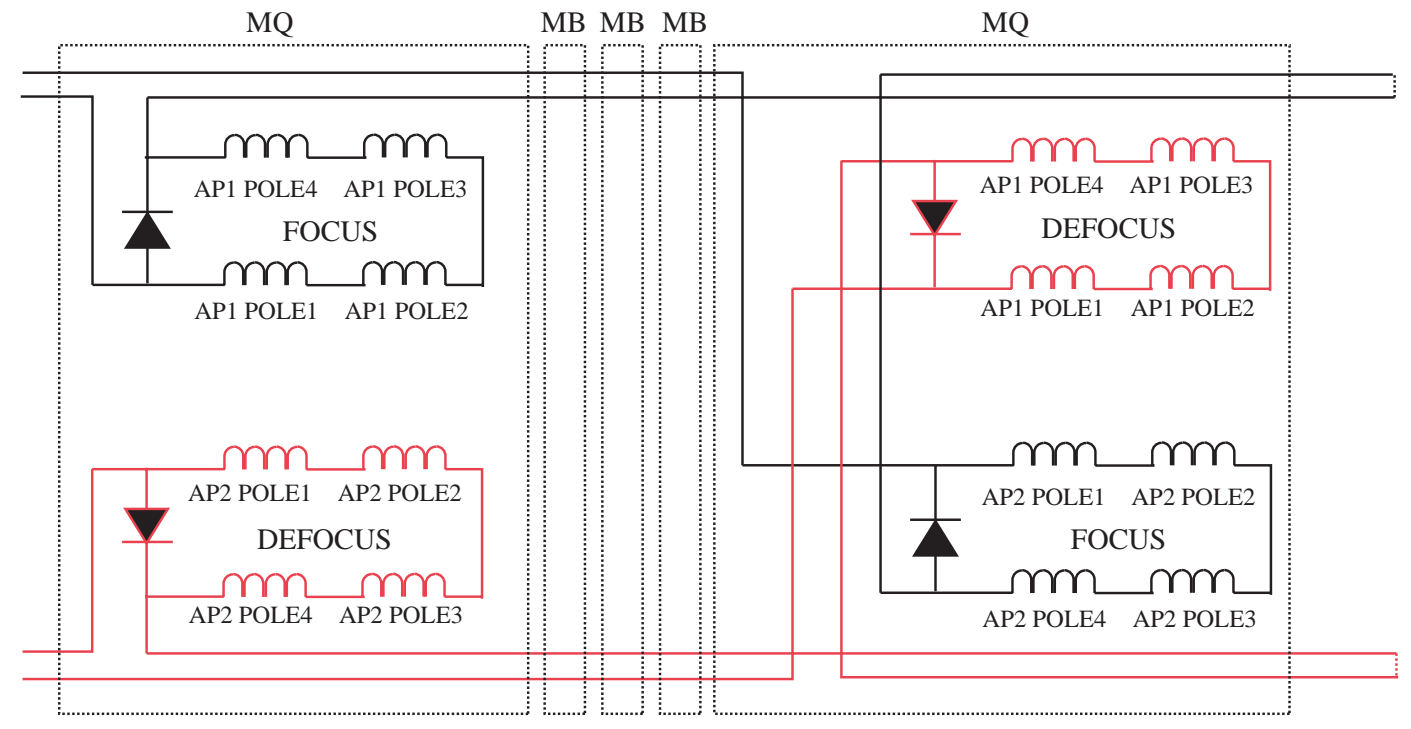

Figure 3.3: Electrical connection of the MQ quadrupoles in one LHC cell.

CMS is shown in Fig. 3.4 [3]. At the collision point the two beams with a transversal dimension around $16 \mu \mathrm{m}$ are crossing at an angle of about $300 \mu \mathrm{rad}$ in order to avoid parasitic beam crossings (collisions with neighbouring bunches). An inner triplet [24, 25] of superconducting quadrupole magnets (MQX) with an aperture of $70 \mathrm{~mm}$ is installed at a distance of about $20 \mathrm{~m}$ from the collision point. The $6 \mathrm{kA}$ superconducting quadrupoles (MQM and MQY) must accommodate separated beams at injection, provide high field gradients (up to $250 \mathrm{Tesla} / \mathrm{m}$ ) and low multiple errors for colliding beams, and sustain considerable heat loads from secondary particles generated in both high luminosity experiments. For the high luminosity insertions (ATLAS and CMS) resistive magnets are used to further separate the beams. For the insertions with ALICE and LHCb superconducting separation dipoles [26] (MBR) and matching sections share the available space with the injection equipment. When the distance between the beams increases to about $190 \mathrm{~mm}$, a superconducting dipole magnet guides the beams into the separate vacuum chambers of the external superconducting quadrupole triplet.

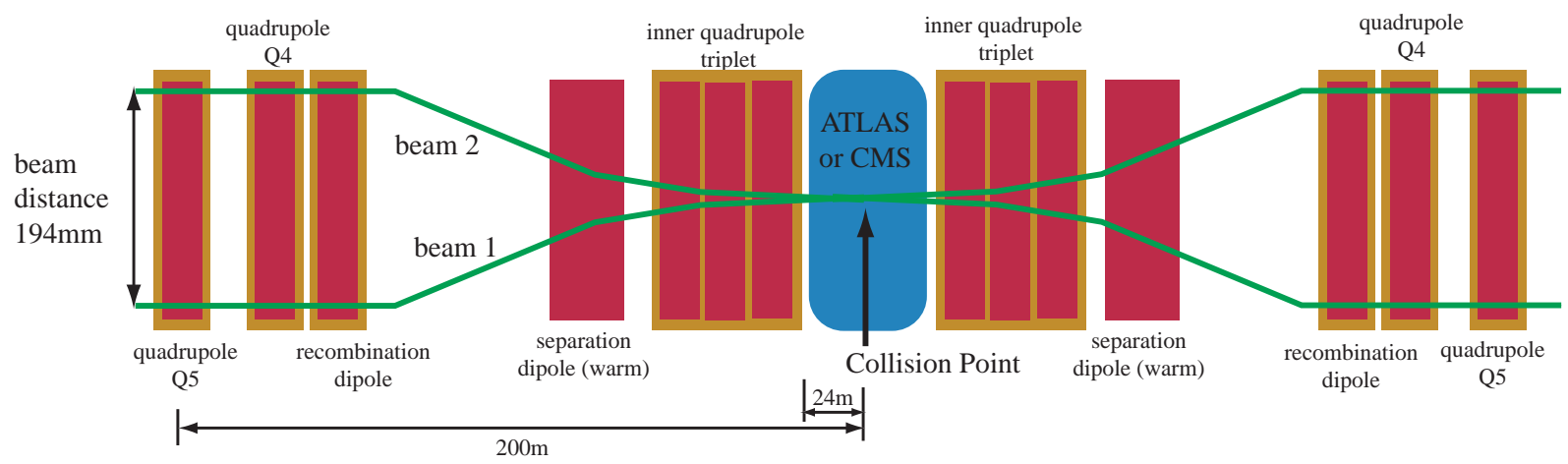

Figure 3.4: Schematic layout of an insertion for the high luminosity experiment [3]. 


\subsection{Corrector Magnets}

The corrector magnets of the LHC are small with respect to the main magnets. They are wound with single strand cables and the coils are fully impregnated with epoxy, which reduces the cooling by helium.

In order to achieve the field quality, sextupole (MCS), octupole (MCO) and decapole (MCD) magnets (spool piece corrector magnets) are installed at the ends of the main dipole magnets correcting the multipole field errors. Every aperture of each dipole magnet is equipped with a sextupole corrector coil, whereas only every second dipole magnet (type B) will be equipped with octupole and decapole correctors.

The sextupole magnets (MS, MSS) correct the chromaticity and the quadrupole magnets (MQT, MQS, MQTL) compensate coupling between the transverse planes and adjust the betatron tune (number of oscillations of the beam around the central orbit per turn). Lattice octupole magnets (MO) will be installed to adjust other beam parameters.

About 1,000 small dipole magnets (MCB in the arcs and MCBC, MCBR, MCBX, MCBY in the insertions) will be installed to correct the particle trajectory in both transverse planes, the closed-orbit corrector magnets.

All the superconducting corrector magnets of the same type located in the same aperture of an arc are powered in series at a nominal current of $120 \mathrm{~A}$ for MCO and 550 A for the rest. The dipole correctors are powered individually with nominal currents from $60 \mathrm{~A}$ to $600 \mathrm{~A}$ depending on their function.

\subsection{Busbars and Current Leads}

The busbars of the LHC are highly copper stabilised s.c. cables that connect all the magnets of a family and attach them to the current leads, which are the feedthroughs from $300 \mathrm{~K}$ to the cold part of the circuit.

\subsubsection{Superconducting Busbars}

The busbars are located in areas of low external magnetic field, the superconductor then works with a larger margin and quenches are less likely than in magnets. Moreover, due to the long decay time of the circuits and the absence of an isolating diode, the copper of the transited part will have to stand the current for a long time, which will require a larger copper cross-section (copper-to-superconductor ratio greater than 10). This implies higher stabilisation, hence lower quench risk.

For the main dipole magnets and the arc quadrupoles, the busbars will carry up to $13 \mathrm{kA}$. They are made of the superconducting cable used to wind the outer layer of the dipole magnets and the arc quadrupoles. The cable is soldered in a copper housing (see Fig. 3.5). The copper stabilisation for the dipole circuit is in the order of $250 \mathrm{~mm}^{2}$ and $160 \mathrm{~mm}^{2}$ for the quadrupoles [18].

For the $6 \mathrm{kA}$ insertion quadrupoles (MQM family and MQY magnets) a special busbar has been developed. It contains copper stabilised strands twisted together with pure copper strands for additional stabilisation.

For the $600 \mathrm{~A}$ corrector magnets two types of busbar cables will be installed in the cryostat. The busbar cable is a strand with a large copper content for stabilisation of about $2 \mathrm{~mm}^{2}$ [19]. 


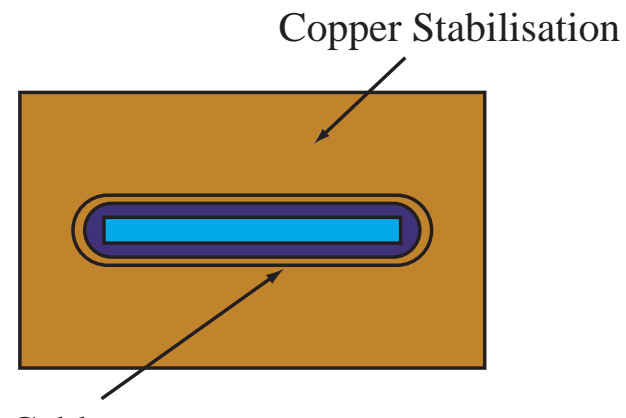

Rutherford Cable

Figure 3.5: Schematic diagram of the busbar cross-section for the LHC main dipole and arc quadrupole magnets.

\subsubsection{HTS Current Leads}

About 3,300 current leads, rated for current from 60 A to $13 \mathrm{kA}$, will power the superconducting magnet system, providing the electrical link between the warm (room temperature cables) and cold (superconducting busbars) electrical circuits.

An extensive R\&D program at CERN has proved the feasibility of incorporating in an efficient and safe way new oxide materials with high $T_{c}$ in current lead applications. Excluding the dipole correctors, which will be powered via specially designed resistive current leads, all the superconducting circuits of the LHC will use HTS technology for their current leads.

The leads are designed to minimise the heat load on the cryogenic system. In order to reduce the heat conduction, the cross-section of a current lead should be as small as possible. However, the reduction of cross-section increases the resistance per length and the heat generation, and reducing the heat generation by increasing the cross-section leads to a larger heat conduction.

The cross-section of a current lead decreases towards the cold end since the resistivity and the heat generation decrease with the temperature. The evaporated helium in the cold mass is used to cool the current lead with forced gas flow along the lead. The use of HTS for a certain length of the current lead reduces the heat generation and enables it to be designed with a smaller cross-section (see Fig. 3.6) with respect to conventional copper current leads [16] relaxing the load to the cryogenic system. A $20 \mathrm{~K}$ helium mass flow of about $0.7 \mathrm{~g} / \mathrm{s}$ is required for operating the lead at nominal current. This flow is regulated as a function of the temperature of the top end of the HTS part [27].

The current leads powering the main magnets in the arcs $(13 \mathrm{kA})$, the different arc corrector families $(600 \mathrm{~A})$ and the quadrupole and dipole magnets of the insertion region (from $3.9 \mathrm{kA}$ to $6.9 \mathrm{kA}$ ) will be grouped in specially designed cryostats, called Distribution Feed Boxes (DFB), located right and left of each of the 8 interaction points.

\subsection{Cryogenic System}

The cryogenic system of the LHC has to produce and distribute the refrigeration capacities required for the different cryo-magnets, accelerating cavities and their ancillary equipment. The general layout is based on a five-feed scheme using eight cryogenic plants, each one dedicated to the refrigeration of one LHC sector. A cryogenic plant is constituted of: 


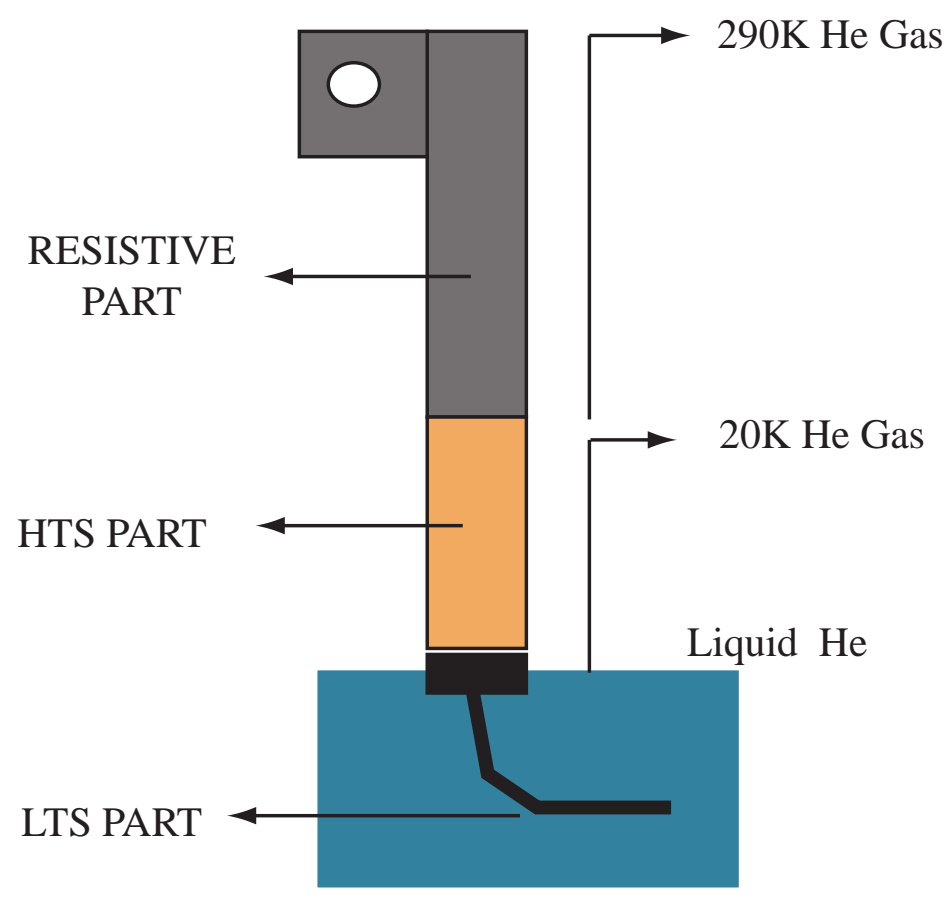

Figure 3.6: HTS current lead [27] (LTS: low temperature superconductor, HTS: high temperature superconductor).

- A $4.5 \mathrm{~K}$ refrigerator with an equivalent entropic capacity of $8 \mathrm{~kW}$ at $4.5 \mathrm{~K}$.

- A $1.8 \mathrm{~K}$ refrigeration unit producing a capacity of $2.4 \mathrm{~kW}$ at $1.9 \mathrm{~K}$.

- A cryogenic distribution line $(\mathrm{QRL})$ that runs in parallel to the machine cryostats. It distributes in the tunnel the cooling power along a complete $3.3 \mathrm{~km}$ machine sector. The latter have a unit length which varies from $107 \mathrm{~m}$ (arc cell) down to $10 \mathrm{~m}$ (stand-alone magnet).

- A cryogenic interconnection box that links the $4.5 \mathrm{~K}$ refrigerator, the $1,8 \mathrm{~K}$ refrigeration unit and the cryogenic distribution line. When possible, this box interconnects also two adjacent cryogenic plants.

Every second short straight section (with the main quadrupole and lattice corrector magnets) is flanked by a cryogenic service module that houses service piping for quench discharge into the helium recovery line and for cooling by pressurised superfluid helium via a link to the QRL.

In order to limit the hydrostatic head due to the tunnel slope and to allow short interventions on the cold mass, a sub-sectorization of the pressurised superfluid helium volume as well as for the insulation vacuum has been made. Busbar plugs have been introduced between arc cell. The cryogenic line has been provided with vacuum barriers after every second cell. Safety relief valves are installed in every cell while cooldown and fill valves every two. In this way only two full cells must be cooled down after a magnet intervention while the rest of the arc stays at cold [28].

\subsection{LHC Quench Protection System}

All superconducting elements in the machine require protection in case of a quench or other failures which may perturb the accelerator operation or even damage equipment. 
The quench protection system of the LHC (QPS) [29] continuously monitors the proper operation (within the superconducting state) of the magnets, current leas and busbars. It is responsible for their integrity in case a transition to the resistive state appears. This main objective is achieved by:

- Detecting the resistive transition and undertaking actions internally to the system or provoking elsewhere the necessary actions through the interlock controllers in order to de-excite the circuits properly.

- Preventing powering if the protection system is not ready.

- Sending alarms during operation to the control room like quench events or important changes in the configuration of the system.

- Being capable of self-testing and of monitoring the state of the elements during operation.

The quench protection is responsible therefore of bringing the superconducting elements to zero current safely. This requires not developing more than:

- $350 \mathrm{~K}$ for magnets with cables of Rutherford type.

- $200 \mathrm{~K}$ for magnets with impregnated coils.

- No quenching for the HTS current leads: they should remain within flux-flow regime.

- $300 \mathrm{~K}$ for all busbars.

- $1300 \mathrm{~V}$ to ground for the main dipoles and $500 \mathrm{~V}$ for the other circuits.

Some elements require a complex protection system, whereas for others the protection can be fairly simple, depending on the design of the elements and their powering scheme. In all of them the quench has to be detected and the power converter switched off. If by switching off the powering, the current goes down fast enough for all the elements in the circuit nothing else must be done (e.g. MCB circuit). If it is not enough, the current must be forced to go faster by warming up the coils (quench heaters) or introducing a resistance in series with the circuit to extract the energy and dissipate it at room temperature (energy extractors). For circuits with large inductance and stored energy this can be not enough and the quenched element (or elements) has to be isolated from the chain (current by-pass). The proper protection strategy must be applied to each element to reach a coherent safety level. Unneeded actions must be avoided in order not to fill up the tunnel with unnecessary protection equipment.

\subsubsection{Protection of Main Magnets}

The longitudinal quench propagation velocity at nominal current for the dipole magnets has been measured to be in the range of $15-20 \mathrm{~ms}$. This low value together with the huge circuit inductance make necessary warming up and isolation of the magnet via quench heaters and a cold diode. The energy extraction will control the current decay ensuring the integrity of the diode and keeping the voltages to ground at a safe value. Lattice quadrupoles will follow the same protection recipe. 


\section{Quench Detectors}

For the LHC main dipole and quadrupole magnets, the detection is based on local quench detectors which are fully redundant and independent of other magnets.

The quench detector for the dipole magnets is based on floating bridge detectors. The voltages across the two apertures are compared. A quench is detected if the absolute voltage difference exceeds a pre-set threshold during a certain time. For the main quadrupole magnets, the comparison is made between two sets of two pole, since the two apertures of the quadrupole magnets are powered by different circuits.

In case of a quench the detector will activate directly the associated quench heater power supplies. At the same time a safety relay will open the respective current loop, which triggers the overall machine protection system.

\section{Quench Heaters}

The quench heaters consist on stainless steel strips of $0.025 \mathrm{~mm}$ thick and $15.0 \mathrm{~mm}$ wide, glued between two layers of polyimide electrical insulation foil of $0.075 \mathrm{~mm}$ each. They are positioned along the magnet between the coil and the collars, the polyimide acts as support and insulates the strips against the coils and the collar structure that is at ground potential. They are heated by capacitor banks that discharge after a quench is detected. The heat is transferred through the insulation layer into the coil, which provokes the transition.

In order to reduce the number of required capacitor banks, heater strips can be partially plated with copper to reduce their resistance. The heat provokes a quench in the cable only below a non plated part and the natural quench propagation drives the entire conductor into the normal conducting state [30].

The location of the quench heater strips on a main dipole is shown in Fig. 3.7. Depending on the magnetic field existing in the area where the strips are located, they are called high-field heaters $(H F)$ or low-field heaters $(L F)$. During the design phase heater strips between inner and outer layer were also considered (see Section 6.3.7).

The copper-plating pattern and the powering of the strips (number of strips per powering bank) has been studied and optimised in [18] in order to guarantee heater efficiency in the entire magnet operating range of the magnet. The strips for the main quadrupoles have a similar design with a $3 \mathrm{~m}$ length and two strips covering each pole.

\section{Quench Heater Power Supplies}

Powering of quench heaters will be performed discharging capacitor banks by triggering thyristors in series with the strips. A capacitor power supply applies a voltage of $900 \mathrm{~V}$ across the heater strips connected in series, providing a peak current of about $85 \mathrm{~A}$.

The heater power supply contains two banks of three capacitors connected in parallel. The capacitors will be operated at $75 \% \pm 5 \%$ of their rated voltage leading to an increased lifetime and a lower leakage current. The thyristors foreseen for the power supplies are intentionally overrated in order to cope with irradiation induced damages of the device parameters.

Every power supply feeds two strips in the case of the dipoles and eight in the quadrupoles (see Chapters 6 and 8). In both cases when the transition is detected the quench is provoked in the two apertures. 
Aperture I

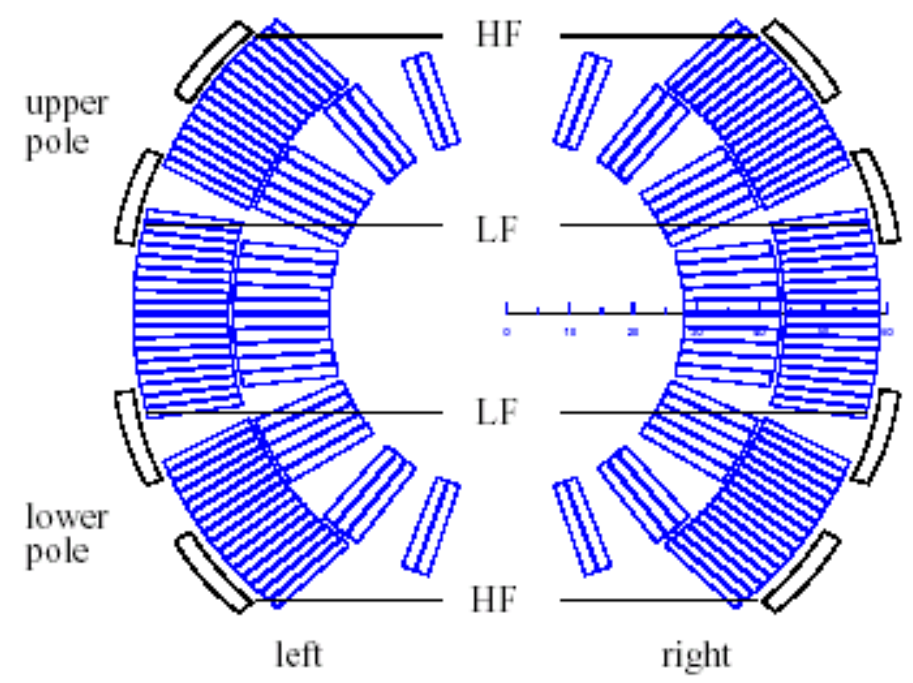

Aperture II

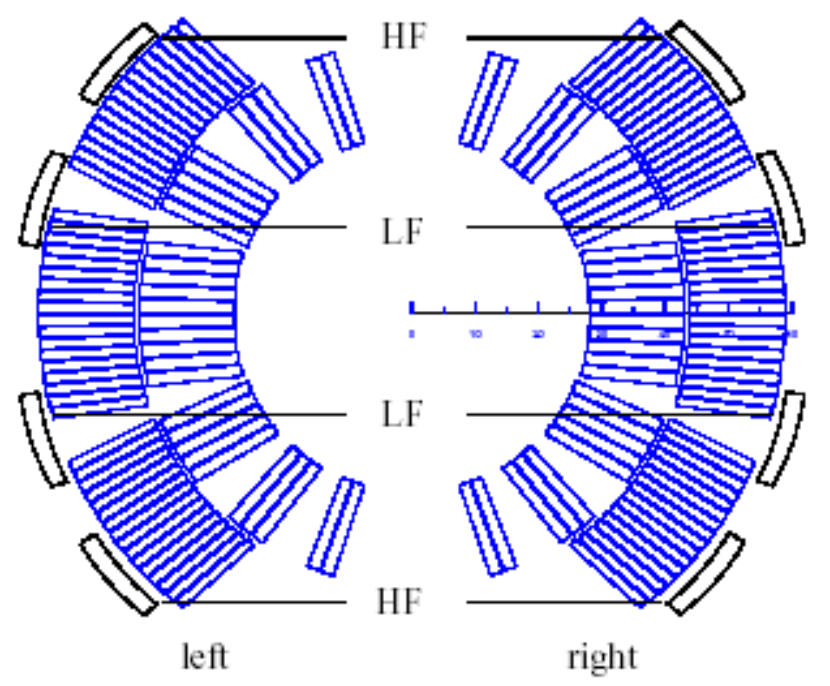

Figure 3.7: Schematic view of the dipole coils with the corresponding high and low field quench heater strips.

\section{Cold Diodes}

All the main magnets in the arcs will be equipped with high-current silicon diodes of the diffusion type, one across each dipole and one across each single quadrupole aperture. Inside the quadrupole diode package the two diodes are galvanically separated due to the independent powering of the circuits.

Each diode package must be able to conduct an ultimate current pulse of $13 \mathrm{kA}$ peak with a nominal decay time constant of about $100 \mathrm{~s}$ for the dipole diode and about $50 \mathrm{~s}$ for the quadrupole ones. The heat sinks for the diode package have to absorb an energy at nominal current of about 1.5 MJ for the dipoles, and $0.7 \mathrm{MJ}$ in the case of the quadrupoles. They have to operate within a temperature range of $1.8 \mathrm{~K}$ to $450 \mathrm{~K}$, withstand the associated thermal stress, and to continue to operate reliably after several cold-warm cycles (endurance tests) before installation.

All diode packages will be tested at liquid helium temperature. As the differences of the electrical characteristics between $1.9 \mathrm{~K}$ and $4.2 \mathrm{~K}$ are rather small, nearly all tests will be carried out at $4.2 \mathrm{~K}$, needing a few of the series diode assemblies to be tested at $1.9 \mathrm{~K}$ to verify their turn-on characteristics [31].

Radiation hardness will be a very important requisite to the diodes due to their special location inside the cryostat (see below).

\section{Energy Extraction}

The $1.33 \mathrm{GJ}$ stored in a dipole magnet circuit are rapidly extracted into two, symmetrically placed energy dump facilities, inserted in series with the two half-chains (see Fig. 3.8). This configuration limits the maximum voltage to ground to $500 \mathrm{~V}$, it halves the energy deposit in each resistor unit and allows symmetrical grounding of the power circuit (ideally, the mid-point of the mid-arc dump resistor). Each of the sixteen main quadrupole circuits will have a single extraction system. 


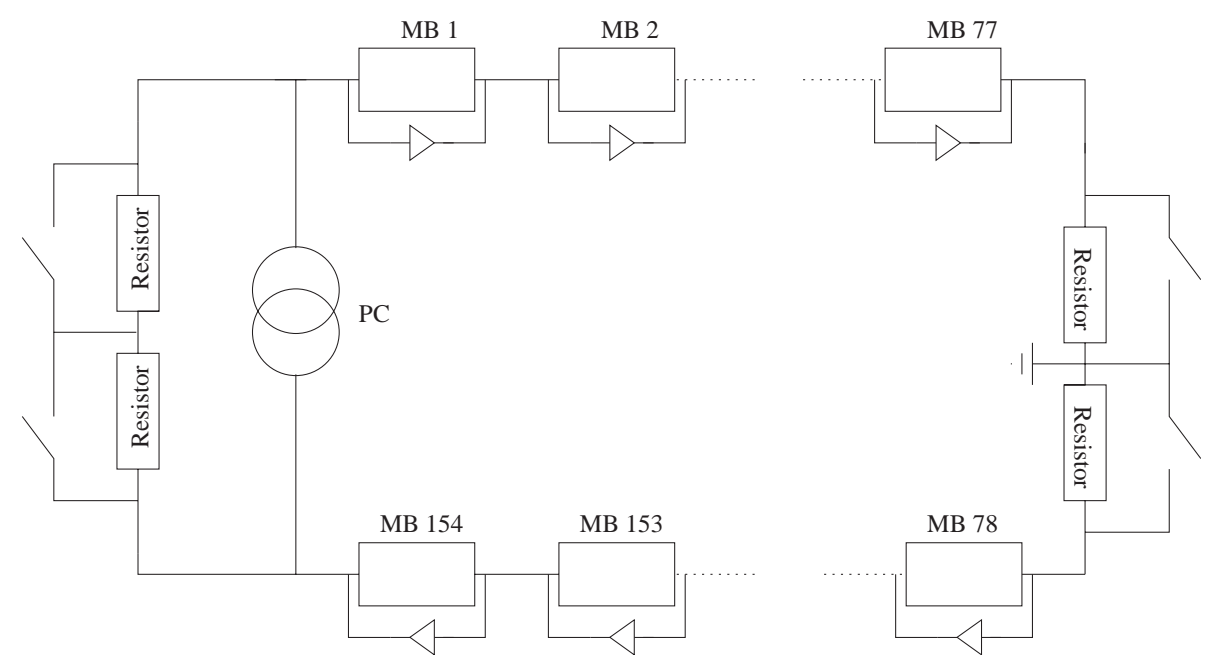

Figure 3.8: Energy extraction location for the main dipole circuits.

The thirty-two installations consist on more than 300 Tm of equipment, including heavy extraction resistors, high-current DC breakers and drivers together with the control electronics. Their main characteristics are listed in Table 3.3.

Circuit Inductance
Stored Energy at $13 \mathrm{kA}$
Extraction resistance
Initial time constant
Maximum current decay rate
Maximum voltage to ground

MD $16.0 \mathrm{H} \quad 263 \mathrm{mH} / 285 \mathrm{mH}$ $1353 \mathrm{MJ} \quad 23 \mathrm{MJ} / 27 \mathrm{MJ}$

$\begin{array}{cc}2 \times 75 \mathrm{~m} \Omega & 6.6 \mathrm{~m} \Omega / 7.7 \mathrm{~m} \Omega \\ 107 \mathrm{~s} & 40 \mathrm{~s} / 38 \mathrm{~s} \\ -122 \mathrm{~A} / \mathrm{s} & -340 \mathrm{~A} / \mathrm{s} \\ 488 \mathrm{~V} & 100 \mathrm{~V}\end{array}$

Table 3.3: Some parameters of the main dipole (MD) and quadrupole (MQ) energy extraction system.

The chosen dump resistance is a compromise between the energy deposition in the cold diode, which will conduct the current in the event of a quench during the discharge, and the maximum admissible current decay rate to avoid magnetic quench-back ${ }^{1}$.

Semiconductor switches were discarded because of their limited radiation resistance, their high on-state losses and because they rely on a single, active opening trigger. Electro-mechanical breakers can be designed with two independent release mechanisms: pulsed trigger and under-voltage release. Moreover they can be installed in the LHC tunnel radiation environment.

The breaker array consists of four parallel branches with each having two series-connected switches each with a current rating of $4 \mathrm{kA}$. A power busway of quasi coaxial structure assures a correct current distribution between the branches.

\footnotetext{
${ }^{1}$ During the current ramps induced currents are generated between the wires and strands of a superconducting Rutherford wire. The copper between the superconductor can be warmed up depending on the current and the cooling conditions. This has a benign effect during quench propagation, since it helps the spread of the resistive part but, on the other hand, can generate quenches during injection and de-excitation.
} 
The extraction resistors have a forced-air cooled, self-inductance compensated stainless steel body and a built-in air-to-water heat exchanger. They will be installed in the tunnel below the beam pipes.

\subsubsection{Protection of the Insertion Magnets}

These magnets (MQY, MQM family and inner triplet magnets) have nominal currents up to $6 \mathrm{kA}$. Voltage taps connected to the midpoints and the cold ends of the current leads will be used to form a floating bridge type detector. In contrary to the main magnets in the arcs, this type of detector will include the superconducting busbars. A Digit Signal Processor (DSP) will allow to implement digital filters and compensation of the inductances.

In case of a quench the detector sends the quench status and quench heater power supplies covering one or more magnets (depending on the circuit) are fired. The heater strips and the power supplies follow the design of the ones for the main magnets described above.

Since these magnets are powered individually or in the worse case in couples, the current drops to zero fastly enough and no energy extraction or decoupling are needed.

\subsubsection{Protection of the Corrector Circuits}

In case the power converter cannot ensure the protection of the circuit, a differential voltage quench detector will monitor the voltage across the whole superconducting circuit. Like for the insertion main magnets, the resistive voltage is computed from the total one (inductive plus resistive) via a DSP. A dedicated current sensor will measure the current and therefore its derivative can be calculated. Inductance table of the circuits must be preloaded in the signal processor.

The protection needs after detection of the quench have been set by simulations [32] for every corrector circuits. Many of the circuits will require energy extraction. The concept for these smaller extractors is based on two, series-connected, fast, mechanical AC breakers, specially equipped with DC arc shutes. The three, simultaneous operated poles are parallel-connected to carry the 600 A DC. For circuits of very low voltage and small stored energy, the energy extraction system will be a part of the power converter. Such systems consist of a combination of a semiconductor crowbar and a low-voltage switch.

For circuits with highest stored energy the quenched magnet needs to be decoupled from the rest of the circuit. Instead of using a cold diode like for the main magnets, a resistor can be mounted in parallel. This cheaper and simpler solution is possible since the de-excitation time for ensuring the integrity of the coil is not as short as it is for the main magnets. In Table 3.4 the needs for each corrector circuit are listed.

\subsubsection{Protection of Superconducting Busbars}

The busbars of the corrector and insertion region magnets are included in the protection of the attached magnet. In contrary, for the $13 \mathrm{kA}$ main busbars of quadrupoles and dipoles in the arcs require a dedicated quench protection. A quench detector is built up with a cluster of digital voltmeters measuring the differential voltage drop at the cold ends of the current leads as well as across selected magnets. The total inductive voltage of the circuit is estimated from the individual lectures by an industrial PC running at real time operation system, which will generate the quench status. After the quench is detected, the power converter is switched off and the energy is extracted from the circuit. 


\begin{tabular}{|c|c|c|c|}
\hline Magnet Family & Magnets per Circuit & Energy Extraction & $R_{\text {parallel }}$ \\
\hline $\mathrm{MCS}$ & 154 & $700 \mathrm{~m} \Omega$ & $80 m \Omega$ \\
\hline MCD & 77 & $700 m \Omega$ & - \\
\hline $\mathrm{MCO}$ & 77 & - & - \\
\hline MO & 13 & $200 m \Omega$ & - \\
\hline MO & 8 & $200 \mathrm{~m} \Omega$ & - \\
\hline MS & 12 & $700 \mathrm{~m} \Omega$ & $150 \mathrm{~m} \Omega$ \\
\hline MS & 10 & $700 \mathrm{~m} \Omega$ & $150 \mathrm{~m} \Omega$ \\
\hline MSS & 4 & $700 \mathrm{~m} \Omega$ & $150 \mathrm{~m} \Omega$ \\
\hline $\mathrm{MCB}$ & 1 & - & - \\
\hline MQT & 1 & $50 m \Omega$ & - \\
\hline MQT & 8 & $700 \mathrm{~m} \Omega$ & $250 \mathrm{~m} \Omega$ \\
\hline MQS & 2 & $50 m \Omega$ (Internal PC) & - \\
\hline MQS & 4 & $700 \mathrm{~m} \Omega$ & $250 \mathrm{~m} \Omega$ \\
\hline MQT & 1 & $50 m \Omega($ Internal PC) & - \\
\hline MQT & 8 & $700 \mathrm{~m} \Omega$ & $250 \mathrm{~m} \Omega$ \\
\hline MQTL & 1 & $50 \mathrm{~m} \Omega$ (internal PC) & - \\
\hline MQTL & 2 & $700 \mathrm{~m} \Omega$ & $200 \mathrm{~m} \Omega$ \\
\hline MQTL & 6 & $700 \mathrm{~m} \Omega$ & $200 \mathrm{~m} \Omega$ \\
\hline
\end{tabular}

Table 3.4: Protection Requirements for the LHC corrector circuits [33]

\subsubsection{Protection of the HTS Current Leads}

The protection system for the current leads is rather simple. Since there is not induced voltage during ramping the quench detector just have to monitor the the voltage drop and compensate the offset voltage. Six voltage taps are installed redundantly at the top, at a midpoint and the bottom of the lead. The power converter is switched off if one of the two voltages exceeds a predefined threshold. In order to cope with the relatively low threshold voltage value of the superconducting part active offset voltage compensation will be implemented in the detector input stage. The current leads are designed to withstand the current decay when the quench is originated in the HTS part.

\subsubsection{Data Acquisition and Monitoring}

The controllers for data acquisition and monitoring (AMC) will observe the status of the protection system, allow for powering, and acquire data for both on-line and post-mortem analysis (e.g. magnet voltages, quench detector signals, signals from the heater power supplies, etc). The AMC units continuously acquire the signals and store them in a circular buffer. When a quench is detected, the acquisition continues during a defined short period of time and then stops. On request, the data is sent to the operators allowing a post-mortem analysis of the event. The integrity of the protection system is monitored, for example whether heater discharge units are not correctly charged or not fired on request, failures in the quench detectors, etc. If a failure is detected, the machine cannot be powered and a message is sent to the operators.

Signals from the QPS are one of the most important groups of the whole LHC Interlock System, which is in charge of controlling the safe operation of the machine. The signals from quench detector, energy extractors and quench heater power supplies are needed for the Power Permit 
of the machine and an important part of them can generate Power Aborts by themselves (see Chapter 10).

\subsubsection{Radiation Tolerant Equipment}

Almost all the electronics needed for the QPS will be installed in the tunnel. This has the important drawback of making the components work in a special environment that in most cases is not the optimum for which they have been designed. The interactions of the beam protons with the residual gas molecules, and particles losses due to collisions with the beam screen (collimation losses) generate a radiation environment that may damage the electronics placed close to the accelerator [34].

Motivated by space and military industry needs, radiation hardened technologies have been developed by some electronic companies. Nevertheless, their application on high energy physics technologies is not very appropriate for two main reasons. First, these rad-hard devices are designed for being used in very well known environments, such as the Van Allen Earth's radiation belts where the geostationary satellites are placed, but they are not supposed to work as well in qualitatively different conditions like those expected in the LHC tunnel. Second, but not less important, the price of these devices is usually some orders of magnitude higher than for the conventional version.

The energy deposition due to the collimation losses must be kept at a low level in order to preserve the superconductivity. This keeps the dose inside the tunnel below 10 Gy per year in the conventional cells [35] (for some places like the low- $\beta$ insertions the dose can be one or two orders of magnitude higher). This dose is mainly generated by neutrons and photons, together with a little amount of protons and charged pions that, like the neutrons, can reach energies up to $30 \mathrm{MeV}$ [34].

Since this relatively low expected dose should not damage the electronics, designers plan to use Commercial Off the Shelf (COTS) components, whose price and availability are much better than rad-hard types. A dedicated test program has been performed during four years at CERN in specially conditioned irradiation areas giving promising results and suggesting hardware changes from the original designs to cope with total integrated doses up to 500 Gy [36].

\subsubsection{Reliable Equipment}

The quench protection system is a key element for the integrity and safe operation of the accelerator, hence its performance will have a very important weight on the total LHC availability.

The system has to be designed to minimise its influence on the total LHC unscheduled downtime. Moreover, QPS plays a very important role on the integrity of the hardware, a single failure in one of its components can lead to fatal hazards that not only will imply considerable waste of time but also huge economical costs.

Protection redundant schemes, component reliability and lifetime, repair policies, checking strategies and required availability are important parameters that have to be considered from the design phase. This is a system that has to work reliably during the 20 year expected lifetime of the LHC and under environmental conditions that will not always be the optimal for its performance and manipulation. 


\section{Chapter 4}

\section{Accelerator Reliability}

\subsection{Brief Reliability History}

Reliability has been appreciated by humans since the ancient times and written references already appear in documents from the Ancient Greece. However, the reliability concept was not applied to technology until World War I. After the conflict it emerged with a technological meaning used in connection with comparing operational safety of airplanes with different number of engines. Reliability was just a measure of the number of accidents the planes had per hour of flight time.

At the beginning of the 30's, Walter Shewhart, Harold F. Dodge, and Harry G. Romig laid down the theoretical basis for using statistical methods in quality control of industrial products, founding the trial and error approach to reliability study. Those methods, anyhow, were not brought into use until the beginning of Wold War II. Products that were composed of a large number of parts, often did not work properly, despite the fact that they were constructed with high-quality components.

A German group working under Wernher von Braun within the project to develop the V-1 missile reported after the conflict that the first 10 missiles were all useless. Despite attempts to provide high-quality parts and careful attention taken to the details of the ensamblation, all of the first missiles either exploded in the launching pad or landed before reaching the British coasts. The mathematician Robert Lusser analysed the missile system and quickly derived the product probability of series components making the first step into the development of the Reliability Theory. This theorem concerns systems functioning only if all the components are functioning and it is valid under certain assumptions. It states that the reliability of such a system is equal to the product of the individual component reliabilities. A system with a large number of components like the V-1 will reach a low reliability level even with very reliable components.

In the United States attempts were made to compensate a low system reliability by improving the quality of the components. That improved the obtained reliability, but still extensive analysis of the problem were not carried out until the next decade.

Reliability studies for complex systems did not start until the end of the 50's and the beginning of the 60's when the research in the United States was concentrated on intercontinental ballistic missiles and space research mainly within the Mercury and Gemini programs. An association for engineers working with reliability issues was soon established. The first journal on the subject, IEEE-Transactions on Reliability, came out in 1963 and the first text books were published.

The extensive construction of nuclear power plants during the 70's makes increase the interest of engineers on comprehensive risk studies. The called Rasmussen report, WASH-1400, was, despite its weakness, the first serious safety analysis carried out in such a complicated system as a nuclear power plant. 
Together with the nuclear energy industry, during the next decades the reliability theory was mainly developed by the needs of space and offshore oil industries. In these fields the systems are expected to work under more hostile and unaccessible environments where low reliability cannot be compensated by extensive maintenance.

During the 90's reliability started being applied in industry management. Global risk management based on the sequence goal-assignment-proof, and risk informed decision-making techniques started being widely used by professionals in very diverse fields like finances, project management or marketing.

Although reliability is at the beginning of the 21 st century a field used in very different domains of human knowledge, and in some of them such as nuclear or space industry of crucial importance, it is just giving its first steps into the wold of high level research. Reliability studies for large scale test facilities such as high energy particle accelerators have not started until this century.

\subsection{Reliability Engineering}

Reliability engineering is a very general concept which is included into other specific fields (e.g. safety, quality, management), and at the same time defines the performance of a system as a whole. Reliability engineering is not only a set of tools to be used by the designers, the users or the managers of a system. It is, above all, the objective for which those tools where made: the definition and control of the system performance.

From this precept, reliability engineering must establish the ideas and tools to be applied during the whole lifetime of a system. It has to specify the expected system performance and work out the optimal design, operation and maintenance in order to achieve the expectation. It is very important not to consider it as an auxiliary method to make the system better or safer, but as the component of the system that explains why it needs the improvement.

\subsubsection{Definitions}

One of the tasks of reliability engineering is the definition of fuzzy terms for making possible the application of scientific tools in order to quantify concepts related to the common sense (e.g. security, worth, reliable).

\section{System}

An engineering system is the set of abstract or physical elements linked in an specific way in order to generate a benefit for the user, which can be an individual, a group of people or just another system. The elements are call subsystems and they can be considered as a system on their own depending on the scope of the study.

\section{Dependability}

Dependability is the qualitative characteristic of a system that represents its capacity to meet an operating demand under adequate conditions and, therefore, the confidence that the device will suitably and safely perform its function. Dependability constitutes an overall and integral view of the interrelated capacities of a system: reliability, maintainability, availability and safety. 


\section{Reliability}

Dependability is a qualitative concept that can be quantified by different parameters. The reliability of a device concerns its ability to operate under designated conditions for a certain period of time or number of cycles. In other words, the reliability of a device at time $t, R(t)$, is the probability of the device to accomplish its task during a time of length $t$.

Although mathematically reliability has a clear definition, system reliability is often understood as synonymous of system dependability. During this work dependability and reliability will be used, like by most of the authors, indistinctly.

\section{Availability}

Availability is the probability that a device will be operational at a given time. The reliability of a system depends on its history and by definition is a decreasing function with time. On the other hand availability function can increase with time and even reach unity at a time different of zero (e.g. repairs carried out to set the device as good as new).

Besides very simple models, availability at a certain moment $t, A(t)$, cannot be expressed analytically if maintenance is considered. Thus mathematically is more used the average availability $\bar{A}(t)$ as a measure of the fraction of time that the item is in operating conditions in relation to total calendar time. This is a very useful parameter to describe and predict long term or steady system behaviour, but it becomes ineffective for predicting number of system failures under demand.

\section{Maintainability}

This is also a very general term that refers to the goodness of the system to be inspected and repaired and the influence of these actions on the reliability and availability.

It can be mathematically described as the probability that a given active maintenance action, for a system under given conditions of use, can be carried out within a stated time interval when the maintenance is performed under stated conditions and using stated procedures and resources [37].

\section{Safety}

Evaluation, prediction, and control of individual and social risks due to the existence of the system. The risk is understood as the frequency at which an individual or a group of people (which can be the population as a whole) sustain a specified level of harm from the realisation of specified hazards.

\subsubsection{Reliability Function}

Let $T$ be a random variable describing the lifetime of a system $\mathcal{S}$. In other words, $T$ is the length of the time interval during which a system $\mathcal{S}$ operates without failure. It can be then stated than $R(t)$ is the probability that $T>t$ or

$$
R(t)=P(T>t)=1-F(T)
$$


where $F(t)=P(T \leq t)$ is the distribution function of the lifetime $T . F(t)$ describes the failure distribution or the failure law of the system $\mathcal{S}$. Considering the lifetime $T$ as a continuous random variable with failure density function $f(t)=F^{\prime}(t)$, the failure rate function $\lambda(t)$ of $\mathcal{S}$ can be defined as

$$
\lambda(t)=\frac{f(t)}{R(t)}
$$

The failure rate can be explained intuitively as $\lambda(t) \delta t$ describing the probability that the system $\mathcal{S}$, having reached the age $t$, will fail during the interval $(t, t+\delta t)$. It takes different names depending on the field of study: decay constant in nuclear physics, force of mortality in finance or hazard rate in risk analysis.

From Eqs. 4.1 and 4.2 and the fact that $f(t)=F^{\prime}(t)=-R^{\prime}(t)$ it can be easily found that

$$
\frac{d}{d t} R(t)=-\lambda(t) R(t)
$$

The integral of this differential equation for $R(t)$, together with the obvious initial condition $R(0)=1$ since the system begins to run at $t=0$, yields

$$
R(t)=e^{-\int_{0}^{t} \lambda(\tau) d \tau} .
$$

Eq. 4.4 shows that the knowledge of the failure rate function is sufficient to determinate the reliability function $R(t)$. Taking into account the equations above together with $f(t)=F^{\prime}(t)$ it can be stated that all the reliability characteristics: reliability function $R(t)$, failure distribution function $F(t)$, failure density function $f(t)$ and failure rate function $\lambda(t)$ can be settled if one of them is known.

\subsubsection{Typical Failure Distributions: The Bath-Curve}

Practical experience shows that the function $\lambda(t)$ qualitatively behaves as shown in Fig. 4.1. Being very similar to the shape of a classical bath-tub, the function in Fig. 4.1 is named the bath-tub curve of reliability theory. The curve may be subdivided into three different periods. The first stage corresponds to the so-called teething or early failures occurred during the debugging time. It represents the failure rate behaviour of a system caused by components which escaped quality control inspections. If during this period the failed components were always repaired or replaced by new ones, the system may then enter in the second period where the failure rate is practically constant with time. This is the random failure period since failures always occur because of some random influences which are unpredictable and make the system memoryless. The length of this period is called the useful lifetime of the system and it is normally considered as the operating time of the device. After this period, deterioration of the system will become significant, entering in the third stage where wear out failures are dominant. When the system reaches this phase special maintenance and repair operations have to be carried out to keep it available and bring it back, if possible, to the random failure period.

The first part should normally be considered as the commissioning time of a system and its actual operation should start when the failure rate is more or less constant. The study must therefore be focused on systems with failure rates that are either constant or monotonically increasing. A failure distribution with such a failure rate is called an IFR failure distribution (distribution with increasing failure rate). 


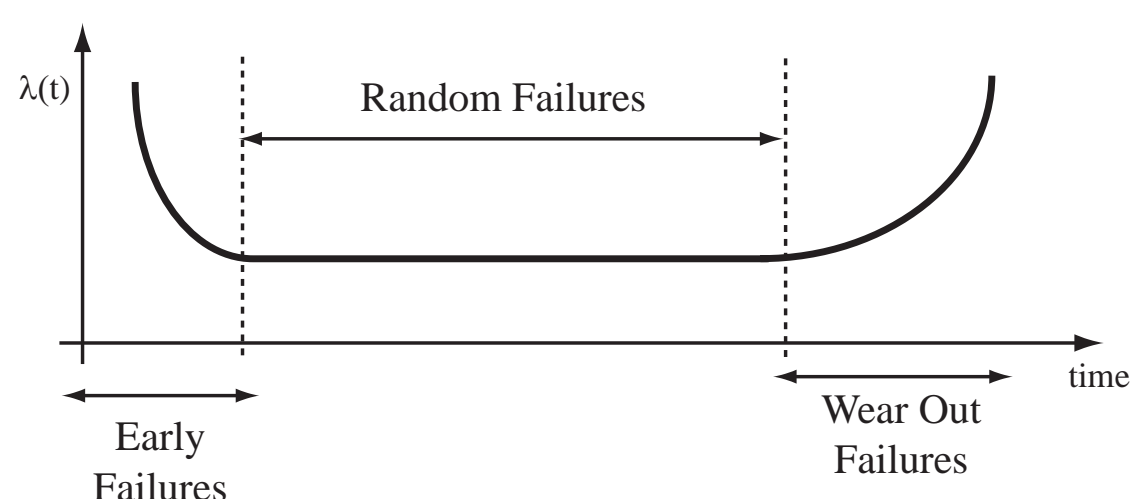

Figure 4.1: Qualitative behaviour of the failure rate function. Bath-tube curve.

The chosen form of the failure distribution will depend on the lifetime period of the modelled device together with other external factors like environmental conditions. The most important failure distributions in reliability engineering are the exponential and the Weibull distributions.

\section{The Exponential Distribution}

Let us assume that the time when the failure of a unit occurs, $T$, is exponentially distributed with parameter $\lambda$. The failure density function is then given by

$$
f(t)= \begin{cases}\lambda e^{-\lambda t}, & \text { for } t>0, \lambda>0 \\ 0, & \text { otherwise }\end{cases}
$$

The reliability function becomes then

$$
R(t)=P(T>t)=\int_{t}^{\infty} f(u) d u=e^{-\lambda t}
$$

for $t>0$. And the failure rate is simply

$$
\lambda(t)=\frac{f(t)}{R(t)}=\frac{\lambda e^{-\lambda t}}{e^{-\lambda t}}=\lambda .
$$

Accordingly, the failure rate of an item with exponential lifetime distribution is constant with time. By comparing with Fig. 4.1 we see that the exponential distribution may be a realistic model for the second period in the bath-tube curve. If $T$ is the failure time of a single item

$$
P(T>t+x \mid T>t)=\frac{P(T>t+x)}{P(T>t)}=\frac{e^{-\lambda(t+x)}}{e^{-\lambda t}}=e^{-\lambda x}=P(T>x)
$$

hence the probability that a unit will be functioning at time $t+x$, given that it is functioning at time $t$, is equal to the probability that a new unit has a time to failure longer than $x$. The remaining lifetime of a unit, functioning at time $t$, is independent of $t$. The exponential distribution has no memory.

Therefore an assumption of exponentially distributed lifetime implies that: 
- A used unit is stochastically as good as new, thus there is no reason to replace a functioning device.

- For the estimation of the reliability function and others, it is sufficient to collect data about the number of hours in operation and the number of failures. The age of the units is of no interest in this connection.

The exponential distribution is the most commonly used life distribution applied in reliability analysis. The reasons are its mathematical simplicity and the realistic models figured out.

If we consider a device with only two possible states: working and not working, a new parameter, which makes reference to the average lifetime of the device, can be defined: the Mean time to failure $(M T T F)$. It can be defined by the expression

$$
M T T F=\int_{0}^{\infty} t d F(t)=\int_{0}^{\infty} t f(t) d t
$$

If the device is in its random failure phase, the mean time to failure is a constant value $\frac{1}{\lambda}$. This is the usual value given by manufacturers and device testers as estimator of a component reliability.

Although MTTF is an essential parameter in reliability analysis it only has a clear meaning when it is applied to single items considered as components of a system. When the whole system is under consideration is worth to speak about mean time between failures $(M T B F)$, since normally a system does not have a unique failure during its lifetime. In other words, the mean time to failure of the components of a system define, by their internal relationships (e.g. redundancies, dependencies, etc), the mean time between failures of the whole system. This concept is explained by different authors as the convenience of MTTF for unrepairable systems and MTBF for reparable ones. It does not make any difference within the mathematical approach if repair times are assumed not to be stochastic.

A system whose components have independent lifetimes following different exponential distributions will not necessarily adopt an exponential failure distribution, actually, it will only occur within the special case in which every component failure causes a system failure, the so-called component series connection.

It is important to distinguish the MTTF (or MTBF) from the useful lifetime. While the first is a statistical value related to a stochastic process and with constant value only during the second phase of the bath-tube curve, the useful lifetime is a parameter independent from the stochastic process that sets the length of time while the exponential model can be applied.

\section{Weibull Distribution}

As explained above, the failure rate $\lambda(t)$ will often decrease in the teething period and increase during the wear out. For some devices (e.g. mechanical units) $\lambda(t)$ will also increase during the useful life period. In such situations the exponential distribution will not be a realistic model.

A distribution often used when $\lambda(t)$ is not constant is the Weibull distribution. Here only its form and main characteristics will be drawn. A detailed overview of its main characteristics together with other functions can be found in the text books [38, 39].

The time to failure $T$ of a device is said to be Weibull distributed with parameters $\alpha(>0)$ and $\lambda(>0)$ if its failure density function is given by 


$$
f(t)= \begin{cases}\alpha \lambda^{\alpha} t^{\alpha-1} e^{-(\lambda t)^{\alpha}} & \text { for } t>0 \\ 0 & \text { otherwise }\end{cases}
$$

where $\lambda$ is called the scale parameter and $\alpha$ the shape. The reliability and failure rate function will be then

$$
\begin{gathered}
R(t)=e^{-(\lambda t)^{\alpha}} \\
\lambda(t)=\frac{f(t)}{R(t)}=\alpha \lambda^{\alpha} t^{\alpha-1}
\end{gathered}
$$

When $\alpha=1$, the failure rate is constant and the Weibull distribution reduces to an exponential. When $\alpha>1$ the failure rate is increasing (IFR distribution), and when $0<\alpha<1, \lambda(t)$ increases with time (DFR distribution). When $\alpha=2$ the resulting distribution is known as the Rayleigh distribution.

Thus, while the $\lambda$ scales the reliability of the device, $\alpha$ sets the different periods of the bath-tube curve.

\subsubsection{Mathematical Modelling of Systems}

The functions defined above depend on statistical values such as the MTTF or the failure rate that can be known with relative easiness for single devices (e.g. electronic components) or very well defined subsystems of an structure (e.g. tank, pump). However, when a larger and more complex system is treated (e.g. car, airport, nuclear power plant), the simple expressions are still useful but the statistical parameters have to be computed taking into account other factors such as the interconnection between components and subsystems, maintenance policies, system operation, etc.

Several powerful tools have been developed during the last century together with the evolution of reliability engineering. The best modelling method (or combination of methods) must be chosen according to the characteristics of the real system, and the objective of the study (e.g. design, maintenance, upgrade, safety, etc)

\section{Failure Mode and Effects Analysis}

Failure mode and effects analysis (FMEA) was developed in the late 50's to study problems that could follow malfunctions of military systems. It is used normally as the first step of a system reliability study. It is based on the review of components, assemblies and subsystems in order to identify failure modes, causes, and effects of such failures.

When criticallicies or priorities are assigned to the different failure mode effects the FMEA is called FMECA (failure mode, effects and critically analysis). Specific worksheets have been developed by different international and local standards to list the component failure modes, effects and other relevant information.

This is a qualitative system analysis and it is really useful when it is done during the design stage of a system. Its purpose is the identification of specific areas where improvements are needed to reach reliability requirements. 


\section{Fault Tree Analysis}

The fault tree technique (FTA) was applied for the first time in 1962 at Bell Telephone Laboratories for evaluating the launching system of an intercontinental missile.

A fault tree is a logic diagram that displays the interrelationships between a potentially critical event (accident) in a system and the reason for this event. The reasons must be environmental conditions, humans error, or component failures.

A fault tree analysis may be qualitative, quantitative or both, depending on the objectives of the study (e.g. listing of possible combination of factors that may result in a critical event, computation of critical event occurrence probability). Quantitative fault tree analysis is throughly described in the literature, see for example [40] and [41]. Its application to fail risk analysis is shown in Chapter 6.

\section{Reliability Block Diagrams}

A reliability block diagram is a success-oriented network describing the function of the system. For systems with several functions, each function is considered individually, and a separate reliability block diagram must be established for each one.

Let's consider a system with $n$ different components. Each component is represented by a block as shown in Fig. 4.2. When there is a link between the end points $(a)$ and $(b)$ the component is said to be functioning. The conditions for such must be properly described. The different components (boxes) are connected to each other forming the system structure. When there is at least one connection between the end points of a structure the system function is achieved, which means that some specified failure modes do not occur.

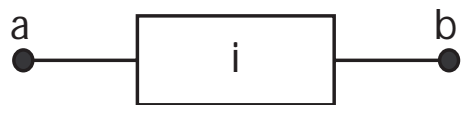

Figure 4.2: Component $i$ illustrated by a block.

A system can be formed by the combination of series and parallel structures. A system that is functioning if and only if all of its $n$ components are functioning is called a series structure. Whereas, a system that works if at least one of its $n$ components is functioning is called a parallel structure. A $k$ out of $n(k-o o-n)$ voting structure is a combination of series an parallel structures where the system works when at least $k$ out of its $n$ components are functioning In Fig. 4.3 a system combining the three structures is shown.

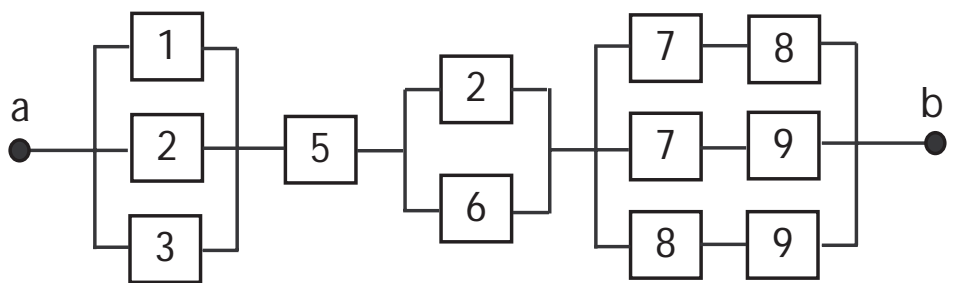

Figure 4.3: System function represented by a reliability block diagram. 
In Fig. 4.3 some components are repeated in the structure. It must be emphasised that a reliability block diagram is not a physical layout diagram for the system, but a logic structure illustrating the function of the system.

Fault trees and block diagrams are equivalent when the tree is limited to OR and AND gates. In order to define a system structure and do qualitative analysis of the system weak and strong components (risk analysis), fault trees represent a more natural approach at least for system that do not have a physical layout similar to a network. FTA is therefore the preferred method. For further evaluations, however, it is often more reasonable to base the analysis on a reliability block diagram. In Chapter 5 blocks diagrams are the starting point for the development of a reliability model for a protection system.

\section{State-Space Method: Markov Models}

The modelling techniques explained so far are based on the assumption that the components can be in one out of two possible states: working or failed. They are also quite static and not well suited for systems where maintenance plays an important role.

Space-state methods are based on the description of a system by its states and by the possible transitions between them. The system states and the possible transitions are illustrated by a spacestate diagram, which is generally called Markov Diagram.

The different states of the system are defined by the states that each component can adopt. Unlike for the previous methods, those states are not limited to binary states, they can have different failure modes or redundancy levels. The Markov diagram is completed with the definition of the possible transitions between states and the occurrence probability of such transitions. The transitions between states are caused by different mechanisms and activities such as failures, repairs, replacements, and switching operations.

The method allows an easy introduction of common cause failures (e.g. storm, fire, quench), which are very important for modelling of protection systems. It is also useful to introduce correlations between components, which was not possible within the methods explained so far since they only applied to systems with independent components.

An important inconvenience of the space-state method is the fast enlargement of the diagram when the system becomes more complex due to the increase of the number of states. Thus the space-state method is suitable only for relatively small or very repetitive systems.

We call Markov process to a stochastic process that satisfy the Markov property:

$$
\begin{array}{r}
P[X(t+\nu)=j \mid x(t)=i ; X(u)=x(u)]=P[X(t+\nu)=j \mid X(t)=i] \\
\forall x(u) ; 0 \leq u \leq t
\end{array}
$$

where $X(t)$ and $x(t)$ are the state of the system at time $t, i$ and $j$ two of the possible states, and $P[X(t)=j]$ the probability of the system to be in the state $j$ at time $t$. In other words, once the present state is known, the knowledge about its future evolution is not altered by additional information about its past behaviour. The conditional probabilities

$$
P_{i j}(t+\nu)=P[X(t+\nu)=j \mid X(t)=i] \quad \text { for } \quad i, j=0,1,2, \ldots, r
$$

with $(r+1)$ being the possible states are called the transition probabilities of the Markov process. 
Finally we define the stationary transition probabilities as those which do not depend on the time $t$ but only on the time interval $\nu$ for the transition:

$$
P[X(t+\nu)=j \mid X(t)=i]=P_{i j}(\nu) \quad \text { for } \quad t, \nu>0 ; i, j=0,1, \ldots, r
$$

A Markov process with stationary transition probabilities is a process with no memory. It is clear the analogy between the stationary probabilities and the exponential failure distribution. Hence this special case of Markov diagrams will be useful for modelling technical systems whose components are in the second period of the bath-tube curve.

A deeper explanation and computation tools of Markov modelling can be found in [42] and [43]. Fault tree analysis, reliability block diagrams and Markov models will be used through this thesis depending on the goal of the study: fault trees for risk analysis and validation, and Markov and reliability blocks for complex reliability modelling.

\section{Monte Carlo Simulation}

An entirely different approach to the system modelling from the methods described so far is based on the Monte Carlo stochastic simulation. The simulation is carried out by generating random and discrete events in a computer model in order to create a realistic lifetime scenario of the system. Once the simulated process have been observed during enough time, estimates are made of the desired measures of performance. Thus the simulation is treated as a series of real experiments.

To state useful and confident inferences about the system reliability, a rather high number of experiments have to be carried out. The number of repeated simulations will depend on the system's complexity and the individual reliability of the components. Systems with high reliability will need more replications than systems with low reliability in order to get equal estimate accuracies. For complex system thousands of replications may be needed with the consequent increase of CPU time.

\subsubsection{Redundancy}

The idea of a redundant system have been already introduced while explaining parallel structures in block diagram analysis. In a structure some components may be of much more importance for the system availability than others. Two ways of ensuring higher system reliability in such situations are to use elements with very high reliability in the critical places of the system, or to introduce redundancy.

Before describing the different kinds of redundancies it is important to know at which system level must the redundancy be applied. A way of making a system redundant would be just constructing two identical systems linked in parallel in a block diagram. Another solution would be to duplicate each component. Both options need the same amount of components but have different reliability improvement or redundancy level. The best solution derives from the following theorem.

Let $\mathbf{x}=\left(x_{1}, x_{2}, \ldots, x_{n}\right)$ and $\mathbf{y}=\left(y_{1}, y_{2}, \ldots, y_{n}\right)$ be the vectors formed by the binary state of the $n$ components of a system and $\phi(\mathbf{x}), \phi(\mathbf{y})$ the state of the system when the state of the components is $\mathbf{x}$ and $\mathbf{y}$ respectively. If we define $\mathbf{x} \amalg \mathbf{y}$ as follows:

$$
\mathbf{x} \amalg \mathbf{y}=\left(x_{1} \amalg y_{1}, x_{2} \amalg y_{2}, \ldots, x_{n} \amalg y_{n}\right)
$$


Where $x_{i} \amalg y_{i}$ is the state of a system formed by two components $x_{i}$ and $y_{i}$ linked in parallel. Since $x_{i}$ and $y_{i}$ are binary variables

$$
x_{i} \amalg y_{i}=x_{i}+y_{i}-x_{i} y_{2}=\max \left(x_{i}, y_{i}\right)
$$

A system $\phi(\mathbf{x})$ is said to be coherent if all its components are relevant and the structure function is nondecreasing in each argument. It implies than when $\phi(\mathbf{x})$ is coherent $\phi(\mathbf{0})=0$ and $\phi(\mathbf{1})=1$.

From Eq. 4.17 we know that $x_{i} \amalg y_{i} \geq x_{i}$ for all i. And from the last definition, when $\phi$ is coherent, $\phi$ is nondecreasing in each argument, and therefore

$$
\phi(\mathbf{x} \amalg \mathbf{y}) \geq \phi(\mathbf{x})
$$

for symmetrical reasons

$$
\phi(\mathbf{x} \amalg \mathbf{y}) \geq \phi(\mathbf{y})
$$

Furthermore $\phi(\mathbf{x})$ and $\phi(\mathbf{y})$ are both binary, therefore

$$
\phi(\mathbf{x} \amalg \mathbf{y}) \geq \phi(\mathbf{x}) \amalg \phi(\mathbf{y})
$$

Eq. 4.20 can be interpreted in common language. Consider the structure in Fig. 4.4 with a system function $\phi(\mathbf{x})$. Assume an identical function $\phi(\mathbf{y})$ with the components in the state $\mathbf{y}$. Fig. 4.5(left) illustrates a structure with redundancy at system level whose function structure is $\phi(\mathbf{x}) \amalg \phi(\mathbf{y})$.

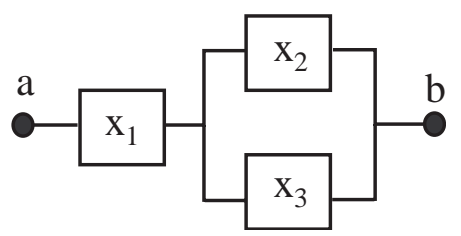

Figure 4.4: Block diagram of $\phi(\mathrm{x})$.

Next consider the system represented in Fig. 4.5(right) where each component pair $x_{i}, y_{i}$ has been connected in parallel. This figure illustrates a structure with redundancy at the component level and the system function is $\phi(\mathbf{x} \amalg \mathbf{y})$. According to Eq. 4.20 the following principle can stated:

A better system is obtained by introducing redundancy at the component level than by introducing redundancy at system level.

The principle is, however, not obvious when the system has two or more failure modes, hence when it is not binary. Redundancy for more complex system is further discussed in Chapter 5.

Redundancies can be divided in two groups: hot (active) redundancy where all the redundant components are in an operational state, and cold (stand-by) redundancy in which the load is not shared by the elements and one redundant component element do not become active until the operating one fails. The redundant components are in stand-by state. An special case of both groups are the voting redundancies where just some of the components must to be working properly $(k$ out of $n$ ) to get the system operational. This kind of redundancy is very suitable for non binary systems (see Chapter 5). 


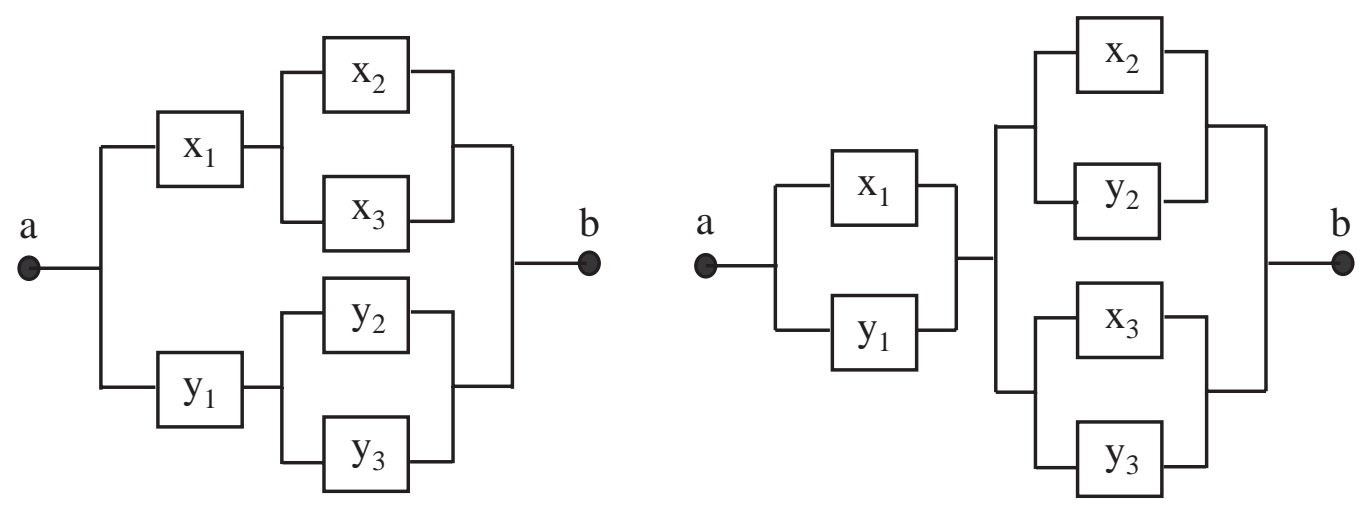

Figure 4.5: Redundancy at system level (left) and redundancy at component level (right).

\subsection{Reliability in Civil and Military Industries}

It was explained in Section 4.1 that the reliability theory has grown up during the last century as an auxiliary field of the space industry and mainly of the military and the nuclear power plants. Afterwards, like most other technologies, it has been applied into more common fields like industrial engineering or finances.

Space, nuclear, and military industries have made the reliability theory evolve to a solid engineering and mathematics subject. At the same time, these fields could plan new goals due to reliability engineering development.

The space and military technologies from a reliability point of view have many common aspects. The most important is the low possibility of maintenance once the system is set into operation (e.g. missile launched, orbit satellite, etc). Although for some projects some repair actions can be taken after they are discovered (e.g. like the repair operations carried out in the main lens of the Hubble telescope), the most part of them collapse if a critical failure occurs (e.g. Ariadne V rocket, Mars Path-Finder) and some of them involve a high risk to humans like occurred in the Challenger and the Columbia spacecrafts, or the Kursk submarine accidents. Moreover, in the few cases like the Hubble for which some repairs are possible, these are extremely expensive thus highly undesirable.

Military and space industries have to face this inconvenience by achieving the highest reliability level from the design phase. The first step for so is to have a very good knowledge of the used components. This is the reason why the most widely used databases about device reliability proceed from these fields. The best example are the different editions of the so-called US Military Handbook [44]. It lists the reliability characteristics (mainly the failure rate) of a huge amount of electronic and mechanical devices under very different operating conditions. It is certainly the most used reliability database of commercial and military electronic devices worldwide, and many of the data used along this thesis come from it.

Nevertheless, the use of these manuals have to be done carefully, taking into account that the given data are test results in certain conditions that, although being properly defined by the author, they can be totally different from the ones in which the studied system is supposed to work. Tools like sensitivity analysis will have to be applied in order to get a reasonable confidence about the expected system reliability.

The necessity of highly reliable components not only led to the detailed elaboration of reliability databases but also to the development of new technologies which were able to fulfil new 
requisites such as radiation environments (e.g. satellites in the Van Allen radiation belts), extremely high temperatures (e.g. H bomb) or very long lifetimes (e.g. Voyager space probes). Some of the new developed devices were made specially as prototypes for the projects, but most of them have been adopted by the private industry and can be found in the manufacturers catalogues. The disadvantage is that the cost can be some orders of magnitude higher than for the conventional devices.

The third key to face the impossibility of maintenance has to do with the system structure. As was already shown by the V-1 missiles during World War II, very high-reliability components do not ensure on their own high reliability of the system. The system has to be able to keep its performance level when one or more failures occur: the system has to be redundant. It has been already introduced in Section 4.2.5 how redundancy has to be applied to complex systems. The increase of needed components will always have an important impact on the final cost of the project, hence redundancy has to be applied in an optimal way. As it will be seen in Chapter 5, redundancy will not always improve the performance of systems with components which can be in more than two possible states. Its application must be carefully studied.

For military and space applications the redundancy is the most powerful way to get the desired reliability level from the design stage. Expected and non expected failures of the components have to be solved a priori, and redundancy is the way to do it. One important inconvenience of this solution for these fields is the consequent increase of the total weight of the system, an essential parameter in all the space projects and most of the military ones.

Nuclear industry has been also the origin of reliability theory, but with a different objective than in the space agencies and the armies. The main goal of nuclear power plants is the production of energy in a very safe way. The safety requirement was already present in the first plants built up during the 60's. Nevertheless, safety became the main objective of occidental nuclear power plants after the Three-Miles Island accident in 1979 and above all after the Chernobyl catastrophe in 1986.

Evolution of reliability theory within the nuclear industry is therefore focused on hazards and risk analysis and safety-oriented designs. Although, like any lucrative activity, nuclear industry must look after effectiveness, the designs and operations in power plants are totally constrained by safety goals: the failure just cannot occur. Redundancy, maintenance and design efforts are addressed to the auxiliary safety systems.

Unlike space technology, nuclear technology can be inspected and repaired. The vital systems of a nuclear power plant are not easily accessible, but critical failures from the production point of view, can be repaired in a relatively short time. Redundancy then will be needed, but if the system is properly instrumented the damaged component can be identified and consequent actions be taken.

\subsection{Reliability of Existing Accelerators}

Although a significant history of accelerators has been accumulated since the first applications around half a century ago, there is a deficiency in reliability estimates of accelerator systems. Past and existing research accelerators were designed and operated towards two main goals:

- Collision Energy: the aim of reaching deeper knowledge about atom and nucleus structure is limited by the centre of mass energy in collision. During the first decades of accelerator application energy was the only research limitation. 
- Luminosity: once nuclear physics moved to particle physics, the interesting events became rare even at enough energy. Luminosity is related to the collision rate, hence to the beam intensity and focusing.

The figure of merit of current accelerators is based in these two characteristics. These machines were conceived as prototypes whose main objective was not the efficiency but to push the performance to the limit.

As a consequence, there is not a developed methodology for a reliability oriented design, since reliability of the system was not considered as major topic and the few efforts carried out in this direction were focused on inspection and repair policies [45, 46].

During the last years, the accelerators have been used for the first time as subsystems of major projects, this is the case of the high power proton accelerators for the Accelerator-Driven Systems (ADS) (e.g. Accelerator for Production of Tritium, Accelerator Transmutation Wastes, International Fusion Material Irradiation Facility). Once the high energy accelerator is linked to an industrial process it cannot be considered as a prototype and needs to perform as well as the rest of the system.

In parallel to these new technologies, synchrotron radiation light sources and accelerators in medicine have become important installations where beams are provided to a large number of users for few expensive hours. In all these cases, beside safety operation, reliability and availability are preferred to peak performance.

These new applications of accelerators outside high energy physics research have increased the efforts of the accelerator's community toward a better reliability data compilation [47] and tool development.

The main ideas about how to face the reliability issues in the new accelerators were presented by different authors during the Workshop on Utilisation and Reliability of High Power Proton Accelerators in Aix-en-Provence in November 1999 and at the First Accelerator Reliability Workshop held at Grenoble in February 2002. These can be summarised in:

- Reliability engineering for accelerators must quantify the common sense given by the broad experience of the research institutes, using established scientific tools together with a clear definition of fuzzy terms.

- The reliability studies of the different systems should not pretend to give absolute value predictions, but they should be conceived as a tool to compare and optimise the possible solutions.

- Reliability and availability are as important as beam dynamics or technology developments and must be integrated in the early design.

- The accelerator community needs the development of common databases and tools to assist on the reliability studies of future and existing particle accelerators.

The main part of the articles are focused on reliability improvement of existing accelerators, and the design-oriented methods were restricted to baseline definitions or small accelerators from which extrapolation to the LHC is not feasible.

Some articles reflect the different attempts to adapt several, very well consolidated methods of the industry and some brother fields like the space industry. The main conclusions that can be extracted from them are: 
- Some Monte Carlo reliability codes have been developed in order to simulate the behaviour of very simple systems under radiation in laboratories not related with accelerators [48, 49]. The main difficulty of accelerator reliability is not the data processing but the data collection. There will be a little difference between analytical and Monte Carlo methods. The higher precision obtained by the latter will be screened by the uncertainty of the input data [50].

- Reliability data from space industry [51] or military [44] is not always valid in accelerator environments. When these data are used for accelerator design the sensitivity analysis of the results to data variability is essential.

- The methods used by the space industry [52] do not fulfil the accelerator design requirements: space engineering is based on very reliable systems due to the difficulty of carrying out repairs, whereas accelerators allow less redundant and weaker designs since the required reliability level can be reached by a proper maintenance policy that must be considered from the early design.

- Failure cost estimation and decision policy can not be based only in economical arguments since the main goals for an accelerator (especially high energy accelerators) are not the same as for industry.

- The safety level of a protection system can be quantified by International Standards [53], extensively used by the industry and from which accelerator technologies should also take profit.

In conclusion, reliability as a systematic tool for accelerator design is just giving its first steps and studies for large scale scientific facilities such as the LHC did not start until this century.

\subsection{LHC Reliability}

The Large Hadron Collider will be the first high energy accelerator in which reliability has been considered as a crucial goal since the design phase.

Superconducting accelerators have already been successfully working for a long time like Tevatron in FNAL and HERA in DESY. The Relativistic Heavy Ion Collider in BNL finished their commissioning two years ago and currently is operating without major problems.

The reason why reliability shows up as an essential tool for the CERN new collider is that, although it is based on very well known superconducting and cryogenic technologies and it can use the experience provided by its predecessors, its complexity makes it a totally different machine. Some of the operational and design challenges regarding the LHC reliability are listed below.

\section{- Required Integrated Luminosity}

Although it is a unique machine, the LHC will not reach its physics goals unless huge amount of data are obtained from it. The rare decays that physicist will search into the LHC experiments occur with a extremely low probability, thus a large number of collisions must be provoked in order to get some few interesting events. The number of collisions after a certain time is related to the integrated luminosity. It is actually the main goal of the LHC. The systems will have to be designed to avoid unscheduled downtimes since they will have a much higher economical and scientific cost than for other accelerators. 


\section{- Energy Stored in the Beam}

The large luminosity required to attain the previous goal together with the huge collision energy leads to beams with large stored energy. The two beams of $7 \mathrm{TeV}$ circulating inside the LHC will carry a total energy of 0.7 GJ. If this energy or a part of it is locally deposited, it can be enough to damage the magnetic components. If the energy is not instantaneously deposited but the losses are distributed along the machine, the accelerator hardware will keep its integrity but some quenches could be generated on the superconducting elements due to particle deposition. The LHC beam will be monitored and actions will be taken to avoid hardware damage and quenches.

\section{- Energy Stored in the Magnets}

The eight LHC main dipole circuits store a total energy of about 10 GJ. This energy is enough to heat up from cryogenic temperature and melt $15 \mathrm{Tm}_{\text {of }}$ copper ${ }^{1}$. The most usual way of this energy to be released is via a quench.

\section{- Number of components}

The extension of the accelerator $(27 \mathrm{~km}$ tunnel) together with the required flexible optics makes the number of critical components required for operation of the LHC much larger that any existing accelerator. Like already occurred with the V-1 missiles of World War II, the very high quality of components will not be enough to achieve the expected availability and redundant systems will be essential.

\section{- Repair Times}

Maintenance in the LHC will not be like in similar accelerators. This is mainly due to the low operation temperature. As was explained in Section 3.4 the cryogenic system sectorisation is designed to enable maintainability with the minimum length of machine warmed up. Still repair times of 10 days have been predicted for interventions that do not require the breaking of the beam vacuum or the venting of the heat exchanger tube (short intervention), and up to 35 days for interventions when the entire continuous arc cryostat has to be warmed up (long intervention).

\section{- Long Beam Dumps}

Even when no repair is needed, unscheduled beam dumps will generate several waiting hours in order to re-establish colliding beam conditions [55]. When the beam dump is provoked by a quench in a main magnet, the recovery time will be between 3 and 8 hours depending on whether the quench is affecting to a single cell or also to its neighbours. This long waiting times after a safe beam dump change the philosophy of beam operation that was applied, for example, at LEP where the experimental conditions could be reached just some minutes after the incident.

\footnotetext{
${ }^{1} \mathrm{~A}$ more revealing analogy is that $10 \mathrm{GJ}$ is around the kinetic energy of the USS Kitty Hawk warrior ship launched at full battle speed of $55 \mathrm{~km} / \mathrm{h}[54]$
} 
Reliability studies will be therefore critical for all the systems related to the protection of the hardware and to minimise the unscheduled downtime. Moreover, and due to the LHC qualification of nuclear installation ${ }^{2}$, the systems related to personnel safety will have to fulfil the safety levels obliged by the Swiss and French legislation. This thesis will focus on the two first areas, however, the introduced tools can be also applied to personnel safety systems.

Although useful information can be obtained from the reliability experience in space, nuclear, and military installations (see Section 4.4), the LHC has many features that call for a different approach to the reliability issues.

\section{- Budget}

This is one of the most important constraints of the reliability of particle accelerators. Nuclear, space, and military industries, due to different reasons, benefit from more relaxed cost margins. This allows the introduction of specially designed devices (e.g radiation hard electronics) and facilitates redundant designs.

Safety requirements in nuclear power plants oblige to high reliability costs, which in many cases can represent the largest part of the whole budget. Space and military applications often require less political justifications about high costs. Projects in high energy physics enjoy less benevolence regarding total costs, and several programmes, like the SSC project in the United States, have been cancelled at an advanced construction stage due to this reason.

Reliability, therefore, will have to be applied to the LHC without compromising the project financial feasibility. Specially designed components and redundancy will have to be applied only where they are absolutely necessary and followed by an optimisation of the functional structure.

\section{- Maintainability}

The main advantage of the LHC machine with respect to the other fields is its high maintainability. The whole system will be fully monitored during operation and technical stops will be performed daily. This allows repairs and replacements where needed. This have a very important impact on the design, since lower redundant level can be afforded in critical points and required reliability be reached by a proper inspection and repair policy.

\section{- Institute Experience}

CERN has constructed and operated accelerators since the early 50's. The big accumulated experience has to be used in any reliability study. A lot of data exist about accelerator component reliability, the new reliability approach has to provide the tools and methods for treating the existing data in a proper way and for ensuring a proper documentation and accessibility of the new generated data.

\section{- Space Availability}

The LHC will use the LEP tunnel and alcoves in order to reduce infrastructure costs, and although some new caverns have been made functional, there is a very important constraint due to the limited space availability. This will reduce redundant possibilities for several systems.

\footnotetext{
${ }^{2}$ Installation Nucléaire de Base
} 


\section{- Data Uncertainty}

Reliability data sources have to be used taking into account the special conditions under which LHC equipment will work. Although the external data can be used as starting point, methods like sensitivity analysis have to be used in order to include their uncertainty.

\subsection{QPS Reliability}

The LHC Quench Protection System represents a key element to prevent hardware damage from possible resistive transitions of the superconducting elements. Besides, it plays a very important role on the unscheduled machine downtime. Around 4,000 signals coming from the QPS systems will be able to generate beam aborts and most of them will be needed to give the green light for power permit (see Chapter 10).

Therefore, QPS will have to be highly reliable since a protection failure will easily provoke irreversible damage on the quenched element, but at the same time, due to the huge number of channels, the signals have to be confident enough in order not to decrease the total available machine time due to spurious protection triggers. A reliability oriented design of the QPS in the LHC must comprise the following phases:

- Experimental and/or theoretical validation of the protection strategies.

- Validation of the used components under the expected LHC radiation environment

- Reliability model of the different quench protection subsystems using existing commercial tools and/or applications developed at the Institute.

- Optimisation of the subsystem designs to minimise risk of hardware damage and unscheduled machine downtime, taking into account:

- Redundant topologies.

- Maintenance policies.

- Spatial constraints.

- Machine operation.

- Cost.

- Outline of the QPS monitoring, definition of protection and warning signals, and planning of repair policies.

- Estimation of the QPS impact on the whole LHC interlock system.

- Analysis of the results' sensitivity to the input parameters such as component reliability data, expected quench rate, or failure costs.

- Validation and correction of final hardware design and maintenance decisions after first months of LHC operation. The huge number of components installed in the LHC will provide enough confident reliability data after few months of operation. 
Along the next chapters the reliability issues of the LHC Quench Protection System are studied. The experimental and theoretical validation of the protection policy is explained and the active subsystems of the different protected elements are analysed in order to optimise its reliability.

Before facing the QPS itself, a general tool for the dependability study of a general protection system has been developed. The author aim has been to create an instrument for assisting in the design of a protection system and, at the same time, to demonstrate the feasibility and utility of reliability studies in the early design phase of an accelerator while providing a new tool to the accelerator community. 



\section{Chapter 5}

\section{Reliability in Accelerator Protection Systems}

\subsection{Protection Systems in Particle Accelerators}

From small medical accelerators to large physics facilities, the special characteristics and working conditions of particle accelerators require a systematic control of the machine status in order to preserve personnel safety, hardware integrity, and facility efficiency.

In the Large Hadron Collider this element is called the Machine Interlock System formed by the the Beam and the Powering Interlock Systems which are in charge of controlling all the subsystems related to the protection of the machine (see Chapter 10): quench protection, beam loss monitoring, beam dump system, collimators, access system, etc.

The whole interlock system will incorporate between 8,000 and 10,000 electronic channels that are able to request a beam dump [5]. This implies that, within the objective of less than one faulty abort every two weeks [56] due to spurious signals coming from these channels, MTBF should exceed 200 years for each channel unless redundancy is included not only for ensuring protection, but also to avoid undesired downtime. The different states that a protection system can show are divided in

- Available - Full Redundancy: the systems is as good as new. This is the level usually exhibited after a repair or at the very beginning of operation after commissioning.

- Available: the system is performing well although some components have failed with consequent loss of redundancy level.

- Safe Failure: generation of one or more false protection signals that trigger the protection procedure. The integrity of the protected system will not be compromised, and the only consequence will be unscheduled downtime.

- Dangerous Failure: the protection system becomes unavailable and the facility unprotected. Although this is a highly undesired situation, this failure has no cost.

- Main Failure: the event (initiator) from which the system has to be protected appears while the system is in the Dangerous Failure state. This is the major failure, the protection system does not accomplish the task for which it was designed. 
A reliability model for protection systems, therefore, will have to take into account the different possible system states and their respective associated costs.

The simulation model should also include the following aspects:

- Inspectionability: the possible system states can be monitored with different degrees of confidence and delay. Some transitions will be noticed immediately whereas others might be transparent to the supervision systems and only detectable during shutdowns.

- Repair Policies: failures can be repaired as soon as discovered, or some delay can be applied according to the spare policy and the admissible risk.

- Failure Screening: component hierarchy within the protection scheme will define the dominant failures.

- Time-Dependant Failure Costs: the consequences of the protection system failure will be different depending on the status of the protected system (e.g. an abort signal will have different running cost impact before injection that during collisions at top energy).

- Failure Dependencies: some failures can occur spontaneously from the full redundant stage, whereas others, like the main failure, have to pass through other failure stages before being reached.

- Sensitivity: the model must estimate the goodness of its predictions by quantifying their uncertainty due to the input parameters, mainly the reliability data and the initiator expected frequency.

\subsection{RESQP: Reliability Model for a Multi-State Protection System}

The objective is to design an analytical model for a general multi-state protection system capable to include the specific characteristics of large superconducting accelerators.

\subsubsection{Safe and Dangerous Failure Reliability}

The relative simplicity of the LHC protection subsystems and the repetitive structure along the machine, make the system suitable to be modeled analytically. On the other hand, the multiple failure modes of the components, the risk and cost time dependencies, and the complex maintenance strategy, recommend the use of Monte Carlo methods. Nevertheless, the high precision of the results obtained with Monte Carlo will fade due to the high uncertainty of the reliability data for accelerator components. This makes the complexity and computing time of the Monte Carlo code (which is the method used by most of the professional reliability software in the market) worthless.

An analytical approach based on Markov Space-State methodology have been chosen. The different states of each component have to be defined together with their transition probabilities and their influence on the state of the complete system. In order to provide a tool for this implementation the simulation program RESQP (REliability Software for Quench Protection studies) has been developed. 


\section{Binary Model}

As starting point the classical analysis method approach for binary systems can be applied. The failure probability of a system $Q_{\text {sys }}$ is related to the failure probability of its cut-sets (components or groups of components whose simultaneous failure leads to a system failure) by the EsaryProschan expression:

$$
Q_{\text {sys }}=\prod_{i=1}^{m} Q_{i}\left[1-\prod_{j=1}^{n}\left(1-Q_{c u t j}\right)\right]
$$

where $Q_{i}$ is the failure probability of the common component $i, m$ the number of common components, $Q_{c u t j}$ the failure probability of the cut-set $j$ excluding the common components and $n$ the number of cut-sets.

Equation 5.1 is very useful while using fault tree analysis (FTA) with common logic gates and for implementing environmental conditions (using the common component states), but it becomes complex when trying to evaluate systems in which common components are not so important and voting ( $k$ out of $n$ or $k-o o-n$ ) gates are often used. In these cases, it is more useful the rare approximation of a system failure probability:

$$
Q_{\text {sys }}=\sum_{i=1}^{n} Q_{\text {cuti }}
$$

which is analog to the reliability expression of a system $R_{\text {sys }}$ with independent components reliability $R_{i}$

$$
R_{\text {sys }}=\prod_{i=1}^{n} R_{i}
$$

Since the reliability of each component (or group of components) can be easily computed and expressed analytically with Bayesian equations, we will only develop the equation for the components linked with a voting gate. This is a parallel system of $n$ elements, here statistically identical, in active redundancy, where $k$ are needed to perform the required function. A trial in which the only outcome is either a certain event (1: "works") or its complement (0: "does not work") can be represented by a random variable called Bernoulli variable. The random variable $\zeta$ defined as the number of ones ("works") with $p$ probability occurring in $n$ Bernoulli trials follows the Binomial distribution:

$$
p_{k}=\mathcal{P}(\zeta=k)=\left(\begin{array}{c}
n \\
k
\end{array}\right) p^{k}(1-p)^{n-k}
$$

For a $k$-oo- $n$ redundancy, $R(t)$ can be interpreted as the probability of observing at least $k$ successes in $n$ Bernoulli trials with a success probability $p=p(t)$.

$$
R(t)=\sum_{i=k}^{n}\left(\begin{array}{c}
n \\
i
\end{array}\right) p^{i}(1-p)^{n-i}
$$


The failure probability for a single component can be computed using the equations introduced in Section 4.2.3. In most of the cases the hypothesis of memoryless components can be assumed. According to the typical bath-tube curve for electronic components, the devices will be always within the random failure period (see Fig. 4.1) since the accelerator lifetime (20 years in the case of the LHC) is usually shorter than the typical edge for this phase (around 200,000 hours). The component failure distribution $p_{i}(t)$ will therefore follow an exponential distribution.

$$
1-p_{i}(t)=e^{-\lambda_{i} t}
$$

This simple model does not include the possibility of carrying out checks, the accelerator status is not taken into account, and only one failure mode can be computed.

\section{Multi-State Model}

Let us come back now to the nomenclature already introduced in Section 4.2.5. The state of the component $i$ is described by a variable $x_{i}$ that does not have to be necessarily binary. The vector $\mathbf{x}=\left(x_{1}, x_{2}, \ldots, x_{n}\right)$ is called the state vector. The system is considered a coherent structure, hence the state of the system can be determined by knowing the states of all the $n$ components. The system is then described by a unique function $\phi(\mathbf{x})$, called the structure function of the system.

The different states reachable by the components and the system are $\mathbf{S} \equiv\left\{s_{0}, s_{1}, \ldots, s_{k}\right\}$ (this indexing is useful for describing a system with $k$ possible failures where $s_{0}$ can be considered the "works" state), and $p_{i j}(t)$ is the probability of the component $i$ to be in the state $j$ at time $t$. Since $\mathbf{S}$ is exhaustive:

$$
\sum_{j=0}^{k} p_{i j}(t)=1, \forall i, t
$$

The device failure probability $p_{i j}$ is computed using the hypothesis of equation 5.6, but now $\lambda$ has to be defined for each kind of failure or state $s$. In this new model state and failure are not completely analogue since the state "works", which is not a failure, is treated like the $k$ "does not work" states.

Once the structure function of the system is set by computing the system state for all the possible state vectors (i.e. using the fault tree analysis), the probability of the system to be at the state $s_{p}$ at time $t, P_{S p}(t)$ can be computed with

$$
P_{S p}(t)=1-\sum_{\Xi} \Phi_{p}(\mathbf{x}) \prod_{i=1}^{n} \prod_{j=0}^{k} p_{i j}^{\delta\left(x_{i}-j\right)}(t)
$$

where $\Xi$ is the space formed by all the possible state vectors $\mathbf{x}, \delta(x-a)$ the delta function in discrete time (Kronecker), and $\Phi_{p}(\mathbf{x})$ the binary function

$$
\Phi_{p}(\mathbf{x})= \begin{cases}0, & \phi(X)=s_{p} \\ 1, & \phi(X) \neq s_{p}\end{cases}
$$




\section{Maintainability}

The maintainability of the system can be included by defining the check vector $\theta=$ $\left\{\theta_{1}, \theta_{2}, \ldots, \theta_{k}\right\}$ as the time between checks for each kind of failure (it has only $k$ elements because the first state is not considered as a failure (i.e. the "works" state). The probability of a system to reach a certain state between two consecutive checks and not reaching such a state in the previous intervals $\alpha_{i}$ is

$$
\alpha_{i}=i P_{S p}\left(\theta_{p}\right)-\sum_{j=1}^{i-1}\left(1-\alpha_{j}\right)
$$

$P_{S p}(t)$ will be therefore

$$
P_{S p}(t)=\sum_{i=1}^{\nu} \alpha_{i}
$$

with $\nu$ being the number of checks carried out since $t=0$. The total reliability of the system will be:

$$
R_{\text {sys }}(t)=1-\sum_{i=1}^{k} P_{S_{i}}(t)
$$

\section{Cost Time Dependency}

Still a last factor has to be included in the model in order to describe the operational state of the machine, in other words, the accelerator status when a failure occurs. A vector $\tau=\left\{\tau_{1}, \tau_{2}, \ldots, \tau_{k}\right\}$ is defined for each possible failure with the total time for every year while the failure can have consequences. It is defined yearly in order to include special periods while the operation of the machine is expected to be different than during the rest of the LHC lifetime (e.g. commissioning and first years at low luminosity). Including this last correction factor, the system reliability will be:

$$
R_{\text {sys }}(t)=1-\sum_{p=1}^{k} P_{S p}(t) \frac{\tau_{p}}{8760}
$$

where $\tau_{p}$ is the total operational time from $t=0$ to $t$ while the state $p$ has a cost.

Although this model looks more complex than the one consisting on independent voting systems with binary output of equation 5.1, its computation is much simpler since, once the structure function matrix is implemented, the code only has to go through all the possible vector state combinations to get each failure probability. Moreover, this model takes into account possible screening between component states (e.g. a failure in a detector channel could lead to a missed initiator, but if at the same time the power supply of a safe-design detector fails, the result will be a false signal). On the other hand, the generation of the structure function matrix can be very time consuming. RESQP includes an auxiliary code in order to assist on this task. 


\section{Example: Fire Alarm}

Let us imagine an office provided with a fire alarm. A very simple model will consider the installation as: some fire detectors and one noise alarm that warns the workers to run toward the closest emergency exit. If the number of fire detectors is two and the alarm is triggered when one of them detects smoke, the number of components of the system will be three, hence the state vector $\mathbf{x}$ has this number of elements:

$$
\mathbf{x}=\left(x_{\text {Detector } 1}, x_{\text {Detector } 2}, x_{\text {alarm }}\right)
$$

Each component can be in different states. The detectors can be perfectly working (available), blind (unavailable) or sending a false alarm (safe failure). The same states can be applied for the other components and the whole system itself.

$$
\mathbf{S} \equiv\{\operatorname{available}(A), \operatorname{blind}(B), \text { false signal }(F)\}
$$

The Markov diagrams are used to define the structure function of the system. In our case, this matrix is formed by rows of four components where the first three define the state of each system device and the fourth sets the state of the system for such situation.

$$
\phi(\mathbf{x}) \equiv\left(\begin{array}{ccc|c}
A & A & A & A \\
B & A & A & A \\
A & B & A & A \\
B & B & A & B \\
F & A & A & F \\
A & F & A & F \\
F & F & A & F \\
F & B & A & F \\
B & F & A & F \\
B & A & B & B \\
A & B & B & B \\
B & B & B & B \\
F & A & B & B \\
A & F & B & B \\
F & F & B & B \\
F & B & B & B \\
B & F & B & B \\
B & A & F & F \\
A & B & F & F \\
B & B & F & F \\
F & A & F & F \\
A & F & F & F \\
F & F & F & F \\
F & B & F & F \\
B & F & F & F
\end{array}\right)
$$


The probability matrix is formed by the device failure probabilities $p_{i j}$. These values, which are time dependent, are computed using the equation 5.6. The rows are formed by the probabilities of a component (D1 and D2: detectors, AL: alarm) for being at each state. The first column is the probability of the element to be available.

$$
p(t) \equiv\left(\begin{array}{ccc}
e^{-\lambda_{B}^{D 1} t}+e^{-\lambda_{F}^{D 1} t}-1 & 1-e^{-\lambda_{B}^{D 1} t} & 1-e^{-\lambda_{F}^{D 1} t} \\
e^{-\lambda_{B}^{D 2} t}+e^{-\lambda_{F}^{D 2} t}-1 & 1-e^{-\lambda_{B}^{D 2} t} & 1-e^{-\lambda_{F}^{D 1} t} \\
e^{-\lambda_{B}^{A L} t}+e^{-\lambda_{F}^{A L} t}-1 & 1-e^{-\lambda_{B}^{A L} t} & 1-e^{-\lambda_{F}^{D 1} t}
\end{array}\right)
$$

In some systems one or more components might not be able to get one of the states defined by $S$, this will be corrected by setting as zero the corresponding element of the probability matrix.

The maintainability vector of this system will be formed by two components: the first will be the time between tests carried out for checking whether the detector and the alarm are not blind and the second will be the time between the checks against false triggers of the detectors or the alarm. Since there is not redundancy on the system against false signals (any of the components can generate the false signal by itself) the second components of the maintainability vector will be infinite (for the calculations it is enough to set a value larger than the expected lifetime of the system). If for example the fire detectors are checked once per year:

$$
\theta=\{8760 h ., \infty\}
$$

Finally the dependency of the failure costs with time can be implemented. If, for example, the fire alarm sounds accidentally when there is not fire, the cost will be different during working hours or during the night. It can be then stated that the two kind of failures will have a cost only during the working hours. If workers are in the office 8 hours per day during the first two years and 24 hours from the third year, and the office opens each day of the year during 5 years, the vector $\tau$ will be the same for the two kind of failures analysed so far:

$$
\tau_{\mathbf{B}}=\tau_{\mathbf{F}}=\{2920 h ., 2920 h ., 8760 h ., 8760 h ., 8760 h .\}
$$

Of course this system could have been modeled in a much simpler way by just using the expressions introduced above for the binary models treating both kinds of failure independently. This would introduce a small error in the final result since the scenarios where the elements are in different failure states (failure screening) would not be considered, nevertheless since only one system is being studied and the failure rate of the electronic devices is rather low, the error would be perfectly negligible. The systems which are object of study in this thesis can be much more complex, and the global failure rate much higher mainly due to the huge amount of subsystems of the LHC. RESQP modeling has the cost of using large-structure function matrices, only manageable with computing codes, but on the other hand provides a robust and systematic way of analysing most of the protection systems included in a high energy physics accelerator. Modeling of very simple systems like the one explained above using both RESQP and the binary model equations have been carried out in order to check the goodness of the results given by the first.

\subsubsection{Renewal Theory: from Dangerous Failure to Main Failure}

The equations introduced above are useful to compute the safe failures (e.g. false triggers) and the dangerous failures (e.g. unprotected system). In a protection system, a dangerous failure generates 
a cost (e.g. accelerator downtime) only when the event from which the system has to be protected (e.g. fire, quench, etc.) comes after the failure occurs and before the next check is carried out.

This problem can be faced in two different ways: the simplest is to use the average availability of the system or, using a more sophisticated approach, the random walk theory. The first method is useful for computing the mean number of expected failures but it becomes too pessimistic when it is used to compute confidence margins. The second method gives optimistic average failures but becomes more realistic for predicting the maximum and the minimum number of failures.

\section{Average Availability Approach}

As it has been already defined in Chapter 4 , the availability $A(t)$ of a system is related to its ability to perform properly its function at a certain moment $t$. In the particular case of protection systems this concept becomes more interesting than the reliability of the system. In continuously working systems, like the accelerator itself, the reliability is the key concept since it will determine the total production (luminosity in this case). However, a protection system which has been operational since its installation and fails just before the initiator appears is as useless as the system that fails when it is installed.

The difference between availability and reliability is introduced by the maintainability of the system. Before any check is done (it can be understood as a check a scheduled inspection or the initiator) the availability is identical to the reliability. When an improvement is carried out, the availability function becomes discontinuous (on its first derivative), rising usually to the unity (i.e. as good as new repair). A discontinuity also appears on the reliability function, but only on its first derivative.

The availability for repairable systems can not be computed analytically and it has to be replaced by the average availability $\bar{A}(t)$. It can be computed at any instant $t$ using Eq. 5.8 and

$$
\bar{A}(t)=\frac{1}{t} \int_{0}^{t} R_{S p}(\tau) d \tau
$$

where $R_{S p}(t)=1-P_{S_{p}}(t)$, being $P_{S_{p}}(t)$ the probability of the system to be in the dangerous failure state $S_{p}$.

For most of the protection systems, periodic tests and replacements are planned. In this case $\bar{A}(t)$ can be approximated by $\bar{A}\left(\theta_{p}\right)$ for all $t$.

If the initiator frequency is assumed as constant with time $\omega_{p}$, the average of main failures $\bar{N}_{p}$ until the first check in $t=\theta_{p}$ is given by the expression

$$
\bar{N}_{p}\left(\theta_{p}\right)=\omega_{p}\left(1-\bar{A}\left(\theta_{p}\right)\right)=\omega_{p}\left(1-\int_{0}^{\theta_{p}} \frac{1}{t}\left(1-P_{S p}(t)\right) d t\right)
$$

In the case of an accelerator with repetitive structure it is interesting to compute the total number of elements (e.g. magnets) that will not be properly protected during the period from the commissioning to the first check, for a certain confidence level $\gamma, N_{p}^{\gamma}\left(\tau_{p}\right)$. This can be computed using the Binomial distribution (Eq. 5.5).

The expected number of main failures after $\nu$ checks, is given by

$$
\bar{N}_{p}\left(\nu \theta_{p}\right)=\nu \bar{N}_{p}\left(\theta_{p}\right)
$$


However, error propagation makes the model too pessimistic when extrapolating $N_{p}^{\gamma}\left(\theta_{p}\right)$ to $\nu$ inspections.

$$
N_{p}^{\gamma}\left(\nu \theta_{p}\right) \ll \nu N_{p}^{\gamma}\left(\theta_{p}\right)
$$

It is certainly necessary in this study to know precisely the statistical error of the results in order to restrict their uncertainty to the reliability data of the components.

\section{Random Walk Approach}

RESQP solves this issue using the renewal methods from Random Walk theory [38]. Let $\xi_{1}, \xi_{2}, \ldots$ be independent and identically distributed positive random variables with distribution $F$ defining a sequence of times by $T_{0}=0$, and $T_{k}=T_{k-1}+\xi_{k}$. If we consider $\xi$ as the time between initiators (renewals), $T_{k}$ will be the time when the $k$-th initiator arrives. Let's define now $N_{t}=\inf \left\{k: T_{k}>\right.$ $t\} . N_{t}$ is the number of renewals in $[0, t], U(t)=E N_{t}$.

$G(t)$ will be the failure distribution of the protection system (computed in RESQP as the dangerous failure probability $P_{S_{p}}(t)$ ), and $V(t)$ the distribution of mean number of initiators after a failure. This defines a delayed renewal process where $G(t)$ is the delay distribution.

$$
V(t)=\int_{0}^{t} U(t-s) d G(s)
$$

If we let $U(a)=0$ for $a<0$, then Eq. 5.24 can be written as $V=U * G$ where $*$ denotes convolution. Applying similar reasoning to $U$ gives:

$$
U(t)=1+\int_{0}^{t} U(t-s) d F(s)
$$

or introducing convolution notation

$$
U=1_{[0, \infty)}(t)+u * F
$$

Convolving each side with $G$ (and recalling $G * U=U * G$ ) gives:

$$
V=G * U=G+V * F
$$

which is our renewal equation. We know [42] that when $G(t)$ is bounded the function

$$
V(t)=\int_{0}^{t} G(t-s) d U(s)
$$

is the only solution of the renewal equation. This integral can be solved analytically if we consider $G(t)$ following an exponential distribution of rate $\lambda$ (this would be the case for a system of devices with constant failure rate connected in series). Initiator occurrence can be considered a Poisson process of rate $\mu$, hence the mean of initiators in $[0, t]$ will be $U(t)=E N_{t}=s \mu$, and $V(t)$ will be then 


$$
V(t)=\int_{0}^{t}\left(1-e^{-\lambda(t-s)}\right) \mu d s=\mu t-\frac{\mu}{\lambda}\left(1-e^{-\lambda t}\right)
$$

A general solution for any $G(t)$ can be solved numerically with

$$
V(t)=\sum_{i=0}^{t / \Delta t} G(t-i \Delta s) \mu \Delta s
$$

Next step is going from the mean $V(t)$ to the probability distribution of initiators after a failure $v(t)$.

$$
\begin{gathered}
d V=\lambda(t) d t \Rightarrow \lambda(t)=\frac{d}{d t} \int_{0}^{t} G(t-s) \mu d s=\mu G(t) \\
v(t)=1-e^{-\int_{0}^{t} \lambda(\tau) d \tau}=1-e^{-\mu \int_{0}^{t} G(\tau) d \tau}
\end{gathered}
$$

Using the nomenclature introduced for RESQP, the probability of getting a main failure before $v_{p}(t)$, when the probability of getting the dangerous failure state, is $P_{S_{p}}(t)$ is given by

$$
v_{p}(t)=1-e^{-\mu_{p} \int_{0}^{t} P_{S p}(t)}
$$

The average number of main failures for the whole accelerators after $\nu$ checks $\left(t=\nu \tau_{p}\right)$ will give a larger value using Eq. 5.22 than applying Bernoulli with 5.33. This is so because the first method considers that the main failure can occur in one element as many times as possible, whereas the second method do not consider multiple occurrence of the same main failure in one element (however, several kinds of main failures can occur in the same element). This sets the important constraint that random walk results will only work when initiators and/or dangerous failures probabilities are low.

On the other hand, since none extrapolation is made, the statistical dispersion is lower using random walk and the extreme values become more realistic. This second method enables the user to introduce non-periodic checks and repairs, hence the number of possible repair policies increases.

\subsection{Sensitivity Analysis}

RESQP computes the sensitivity of the results to the most uncertain parameters, using the Fourier Amplitude Sensitivity Test (FAST). This is a procedure that has been developed for uncertainty and sensitivity analysis. It provides a way to estimate the expected value and variance of the output variable and the contribution of individual input factors to this variance. The main advantage of this method is that the evaluation of each factor influence can be carried out independently for each factor, using just a single set of runs because all the terms in a Fourier expansion are mutually orthogonal 
The main idea behind the FAST method is to convert the k-dimensional integral in the space of all the possible values of the factors taken into account into a one-dimensional integral in $s$ by using the transformation functions $G_{i}$ for $i=1, \ldots, k$, namely

$$
x_{i}=G_{i}\left(\sin \omega_{i} s\right)
$$

where $s \in(-\pi, \pi)$ is a scalar variable and $\left\{\omega_{i}\right\}$ is a set of integer angular frequencies. We are not interested here in developing a deep understanding of the exact influence of each factor on the total reliability, but in reaching a quantitative idea of the confidence and stability of the results presented above. Hence, we will not discuss the different sensitivity estimates.

A suitable transformation $G_{i}$ should provide a uniformly distributed sample for each factor $x_{i}$ in the unit k-dimensional cube $\Omega^{k}$. Several proposal transformations can be found in [57]. Here we will use the one proposed by A. Saltelli in [58].

$$
x_{i}=\frac{1}{2}+\frac{1}{\pi} \arcsin \left(\sin \omega_{i} s\right)
$$

The search curve oscillates over the range of $s$. As $s$ varies, all the factors change simultaneously (in this case, as a set of straight lines oscillating between 0 and 1) and their range of uncertainty is systematically explored. The curve drives arbitrarily close to any point of the input domain if and only if a set of unconmensurate $\omega_{i}$ frequencies is used (i.e. none of them can be obtained as a linear combination of the other frequencies with integer coefficients). In this case the curve is said to be space-filling.

As example, the frequencies $\omega_{1}=11$ and $\omega_{2}=13$ have been chosen. The Figure 5.1 (left) proves that these frequencies are space-filling with transformation of Eq. 5.35 when 630 points are sampled. It also shows (right) which would be the effect of not using unconmensurate frequencies.
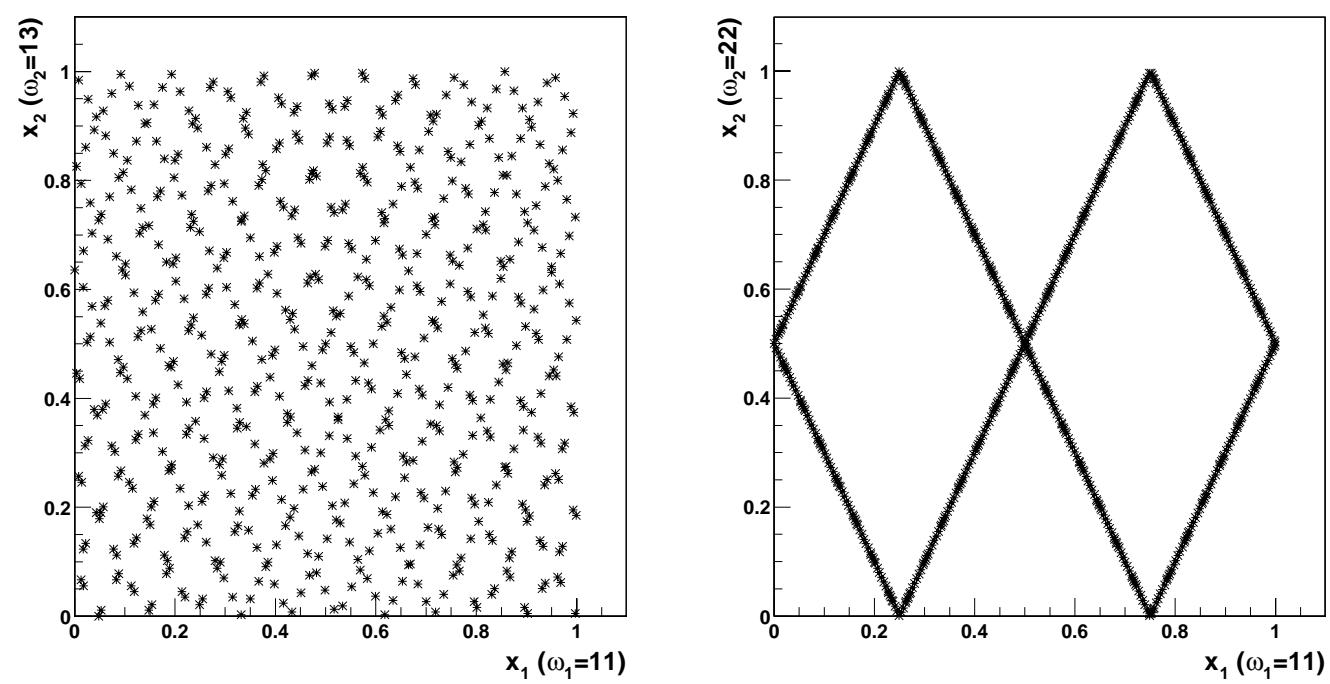

Figure 5.1: Scatter plots of 630 sampling points in a two-factor case with unconmensurate (left) and nonunconmensurate (right) frequencies. 
In Fig. 5.2 a result example is shown. It represents the number of lost dipoles after 20 years in the LHC due to problems in the heater power supplies with respect to the expected quench rate and the electronic component failure rate deviation from the nominal value, for two different maintenance policies.
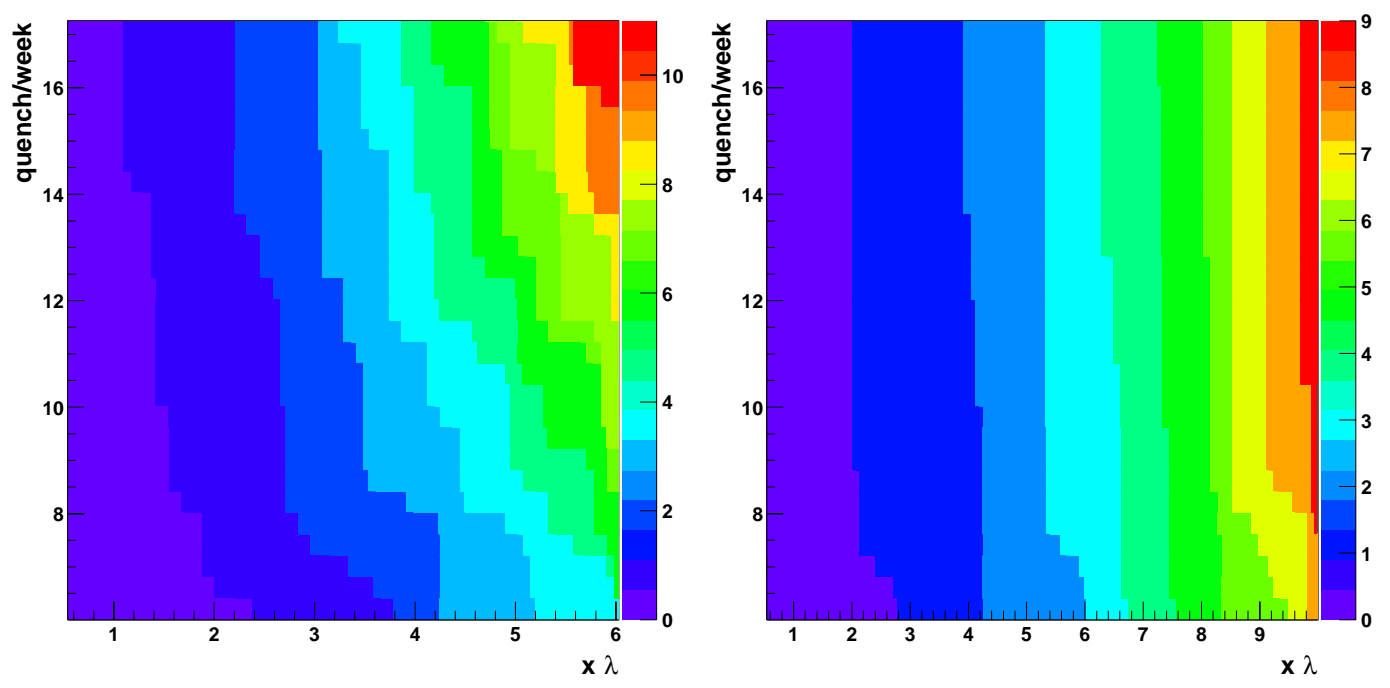

Figure 5.2: Sensitivity of maximum number of lost quadrupoles due to quench heater power supply unavailability ( $90 \%$ confidence level) to component failure rate and quench rate with post-mortem information considered (right) and not considered (left).

\subsection{RESQP execution}

The failure rate data (Weibull or exponential) and inspection frequencies have to be defined for each component and kind of failure. The machine operation time and the periods while a certain failure can generate a cost have to be introduced for each year. This enables the user to include special years such as commissioning at not full luminosity. These data together with general parameters (e.g. accelerator lifetime, expected initiator rate, etc) can be easily introduced using common data management packages such as Microsoft Excel or Origin.

The complexity of the structure function matrix depends on the reliability topology of the system. An auxiliary code included by RESQP assists the user during its generation.

The input data are read by RESQP at the beginning of the simulation run. First, the safe and dangerous failure probabilities for a single element are computed and stored in a file in function of time. The generated file is used by the program to make the integrations needed to compute the main failure probabilities.

The average, minimum and maximum expected failures are computed for the confident interval given by the user. Finally, if required, sensitivity analysis of the results are performed with respect to the desired parameters and within different ranges.

The results are optimised to be treated by powerful scientific data processors like ROOT and PAW. 


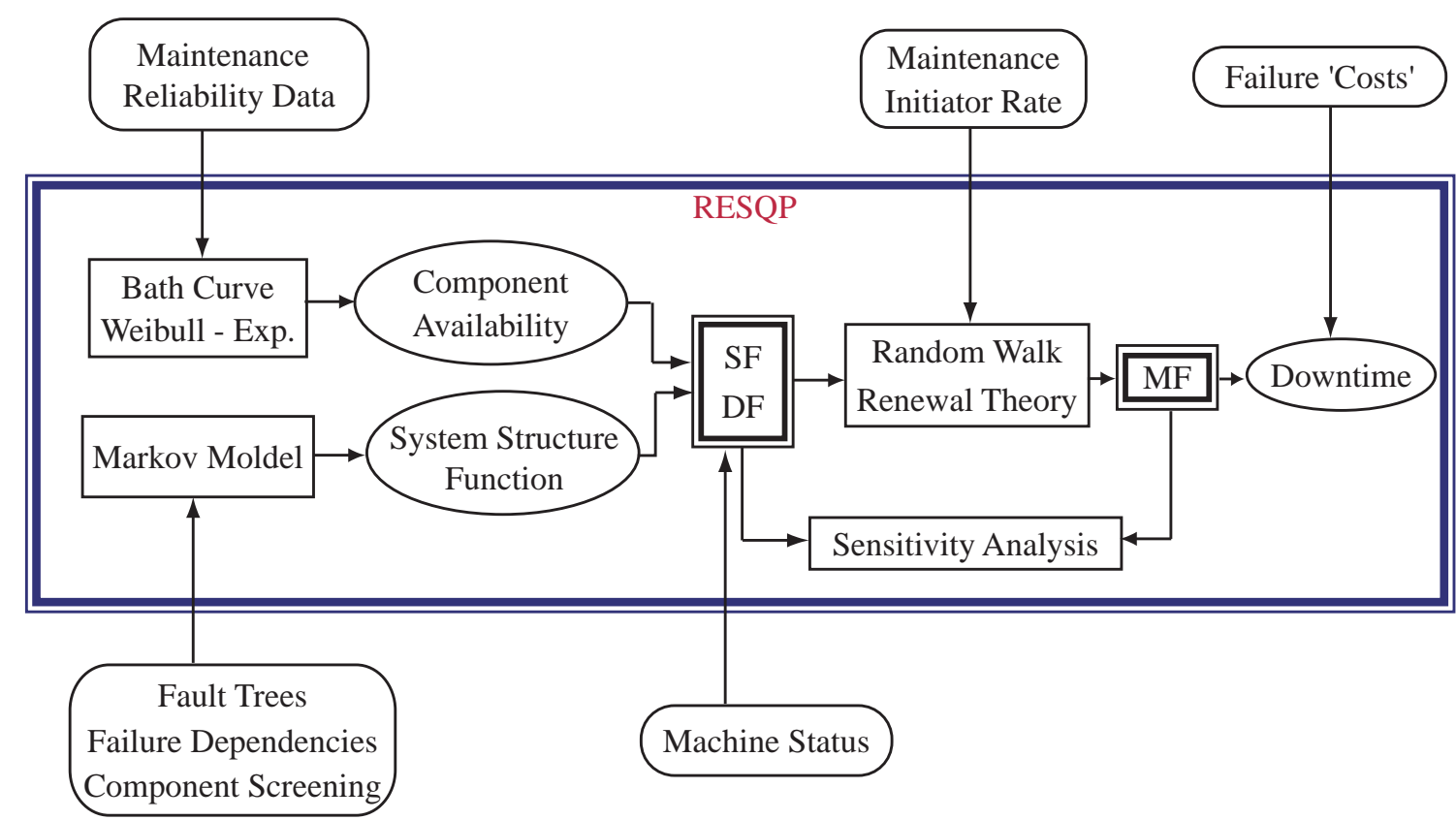

Figure 5.3: RESQP execution steps (SF: Safe failures, DF: Dangerous failures, MF: Main failures).

The program execution times mainly depend on the number of treated elements (usually in the order of some thousands due to the large number of LHC magnets), the random walk integration and the sensitivity computation. The time due to these two last factors can be considerably reduced if computations are made for predictions after a certain operation time (e.g. LHC lifetime) instead of looking for their time evolution. A basic diagram of a RESQP simulation run is shown in Fig. 5.3.

\subsection{Component Reliability}

The reliability of the system components depends on the failure distribution parameters ( $\lambda$ in the case of exponential distribution and $\lambda$ and $\alpha$ for Weibull), and on the length of time while they are applicable.

The failure function parameters are given by the bibliography usually as a constant failure rate (exponential distribution function). It will be useful during the random failure period of the bath-tube curve. In electronic devices this period usually longs in the order of 200,000 hours. Nevertheless this value can be drastically reduced when the device has to work under hostile environments (e.g. radiation, low or high temperatures, etc). In the LHC tunnel, radiation will be the most important inducement of early degradation.

\subsubsection{Radiation Influence on Component Reliability}

Radiation has an important effect on electronic components. The induced damage can be separated into two categories: the single event effects mainly generated by the electron-hole pair creation and the atomic displacement due to the integrated dose. 


\section{Single Event Effects (SEE)}

These are individual events that occur when a single incident particle deposits enough energy to cause an effect in the device. They can be provoked by several natural sources such as the galactic cosmic rays and solar wind flux, formed mainly by protons and heavy particles trapped in the Van Allen belts. For this reason, these effects have been widely studied by the space industry.

Many tests have been done and experimental data can be found in the literature [59]. Unfortunately, the same cannot be said for the damage caused by a radioactive environment like the expected in the LHC. In the tunnel the fast neutrons will be the main responsibles of the SEE, whereas the neutron fluence is almost zero in radiation belts and cosmic rays. Atmospheric neutrons observed at an altitude of $15 \mathrm{~km}$ have a pick fluency below $1 \mathrm{~cm}^{-2} \mathrm{~s}^{-2}$, and they have been recognised as a potential source of SEE for avionics. This level, however, is five orders of magnitude lower than the one expected in the LHC tunnel, and no systematic study of induced-damage on commercial components (COTS) is available.

The damage appears with the creation of a high pair density along a track, which is the result of the protons, alpha particles, ions, recoil atoms, nuclear reaction decay elements, and all the heavy particles whose ionising power is high enough to generate plasma along the track where the particle has crossed the device through. This generates a current of charge injection, which will generate a SEE if the collected charge from the ion strike exceeds a minimum value called the critical charge.

The presence of fast neutrons and protons in the tunnel, which are able to generate highly ionising particles inside the silicon (above all heavy recoil nuclei such as ${ }^{25} \mathrm{Mg}$ ) can be a real problem for the proper performance of the electronics, since each one of them must be considered as a potential SEE. The SEE can be divided in three basic categories [60].

- Transient effects, such as single event upset (SEU) and multiple bit upset (MBU), which change the state of internal storage elements, but can be reset to normal operation by a reinitialisation or other electrical operations.

- Potentially catastrophic events, such as single event latch-up (SEL) and snapback, which may cause destruction unless they are corrected in a short time after they occur.

- Single event hard errors (SHE), which cause catastrophic failure of a single internal transistor within a complex circuit. This can be generated by two mechanisms: microdose deposition in the gate region and gate rupture.

The single event effect hazard cannot be ignored by protection system designers since it can make the device become unavailable from the very beginning of the accelerator operation. It has been demonstrated in [34] that all electronic equipment planned to be installed in an accelerator environment has to be tested and qualified. Space and military studies can be used for a first pre-selection but no definitive decision can be taken without testing the devices.

Nevertheless, these failures are typical teething failures (early failure period in the bath-tube curve) that will be noticed and replaced during the machine commissioning. Hence, single event effects will not have any important influence on the total lifetime (random failure period) of the devices. 


\section{Integrated Dose: Atomic Displacements}

Unlike the SEE, the total ionising dose generates a cumulative long-term degradation, which creates a drift on the electrical characteristics of the irradiated device. It results in gradual degradation of the electrical properties of an integrated circuit due to the total dose or fluency.

The continuous degradation of electronic devices is mainly due to atomic displacements. The semiconductor materials germanium and silicon belong to the carbon family. The crystal structure of these materials can be exemplified by the diamond lattice. An electron cloud surrounding a single atom differs from that of the electron cloud in the lattice atom. The valence band drops to states that are much closer to the inner shell. During the process, energy is released in the form of heat. The amount of energy lost by the valence band electrons in the formation of the crystal is a measure of the binding force of the structure. This energy, called the sublimation energy, goes from 3 to $6 \mathrm{eV}$ [34]. However, an energy approximately four to five times bigger is needed to remove an atom entirely and irreversibly from its lattice site in the crystal structure. In radiation effects terminology, it is known as the displacement threshold energy.

The origin of these atomic displacements are particles like neutrons, protons or nucleus, which interact with the device with enough energy for provoking the different mechanisms that can modify the structure of the crystal: elastic scattering, inelastic diffusion, or nuclear reaction. Since these mechanisms are mainly driven by the strong nuclear force, photons should be excluded, however, high energy photons can produce secondary particles that can create displacements. The atomic displacement can generate four different effects on the semiconductor:

- Decrease of minority carrier lifetime, which is a very important characteristic for solid-state devices. It mainly affects to the bipolar transistors due to the radiation-induced increase of the bulk component of the base current.

- Decrease of the effective majority carrier concentration, which mainly affects to optical properties of the material. In general a decrease in carrier concentration will increase the transparency of a semiconductor on the wavelength region beyond the fundamental absorption edge. This region includes the infrared frequencies that is essential in some applications such as electrical isolation for signal transmission. It has also an important influence on zener diodes, which increase their bias function breakdown voltage.

- Decrease of carrier's mobility. It is an effect that tends to enfeeble the variation of minority carrier's lifetime. This effect predominates at high doses and affects on the resistivity of the semiconductor.

- Creation of acceptor levels, which van generate a type inversion of the semiconductor (n to p). This effect is significant in silicon detectors under high neutron fluency. It is not significant in usual transistors.

The progressive damage caused by the integrated dose will be reflected in the drift of some device characteristics, which will end up in an earlier failure of the device. Although, in general, they will not change their random failure rate, they will modify the length of time while the given values are applicable. Tests have been done in the LHC in order to check the expected lifetime of the components planned to use (see Chapter 6). 


\section{Annealing}

The annealing is the partial or total self-healing of an electronic component or system after exposure to damaging nuclear radiation. In the case of neutron irradiation, most of the displaced atom defects resulting from incident neutrons are not stable at room temperature. This implies that thermal motion will anneal many of the defects. However, these defect atoms can also form associations with impurities in the lattice, which are also stable at room temperature. Hence, annealing can cause improvement in the post-irradiation integrity of the lattice through vacancy-interstitial recombination, but also the formation of stable impurity defects and vacancies that can imply further degradation.

Transient or rapid annealing of semiconductor device performance degradation following pulsed neutron exposure is predominantly a bulk effect. Annealing occurs in neutron-irradiated transistors over a whole range of temperatures above and below room temperature. However, in order to get full annealing, the temperature must be higher than the maximum working temperature of the device, which of course will never happen in the LHC tunnel in normal conditions.

\subsubsection{Other External Factors}

Although radiation environment is the most important source of damage to equipment (and above all electronic equipment) around an accelerator facility, other external factors cannot be ignored.

These other damaging sources are mainly: temperature, humidity and chemical pollution in the tunnel. There should be also considered the working conditions of the devices such as the voltage and current rating.

For the LHC Quench Protection System equipment, some tests have been carried out in order to validate the performance of the most sensitive components to these factors, such as the capacitors of the quench heater power supplies (see Appendix A).

\subsubsection{Failure Distribution Data}

The external environmental and operation conditions explained above may influence on the total lifetime of the components. Hence, their influence of the availability of a protection system is easily controlled if tests are made before installation and a proper monitoring of the device state is carried out during operation. For the cases whose lifetime is shorter than the accelerator lifetime, the failures will appear more or less at the same time and a general update of the system will be required. This can have an important cost on the machine operational budget, but the influence on the unscheduled downtime will not be important since the failures will be already expected.

On the contrary, the random failures occurring during the second period of the bath-tube curve will be the cause of most of the unscheduled beam dumps and hardware damages provoked by failures on the protection systems. It is essential for a reliability study to estimate the failure probability of every component. The main data sources used by the reliability engineers are the US Military Handbook in its different versions and the data provided by the manufacturers.

The Military Handbook [44] is a reliability database developed by the US Department of Defence with the objective of establishing and maintaining consistent and uniform methods for estimating the reliability of mature design components used by military electronic systems and equipment. It provides the formulae and data to compute the failure rate of the devices taking into account the nature of the device and the operating conditions. It does not evaluate the influence of these conditions on the total lifetime but only the effect on the random failure rate. 
The other main source of data are the information coming from tests carried out by the manufacturers. In this case, data are provided for a certain commercial device, whereas the Military Handbook does not take into account differences between producers and only the used technology is consider. Thus confidence of data for a certain device is higher. However, these reports usually only provide information about the test results under certain conditions (typically $125^{\circ} \mathrm{C}$ with a $60 \%$ confidence level), and values have to be modified in order to estimate their performance under the operational environment.

This extrapolation to different confidence level and/or temperatures can be easily done when enough data are given about the test conditions and results.

\section{Confidence Level}

To obtain failure rates at different confidence levels, it is necessary to make use of specific probability distributions. The chi-square $\chi^{2}$ distribution that relates observed and expected frequencies if frequently used to establish confidence intervals. The relationship between failure rate and the chi-square distribution is as follows:

$$
\lambda=\frac{\chi^{2}(1-\alpha, \delta)}{2 t}
$$

where $\alpha$ is the confidence level, $t$ the total device-hours of the test, and $\delta=2 r+2$, being $r$ the number of failures, is the degrees of freedom.

Life tests are based on counting the number of failures for an amount of device-hours in order to figure out the distribution of failures. The minimum value must be zero for no failures and the maximum corresponds to $100 \%$ failures, however it can be assumed that the number of parts under tests is high enough in order not to observe failures in the whole sample.

The factor of two in $t$ and $\delta$ is necessary to achieve theoretical validity of the $\chi^{2}$ distribution (see Appendix B). Given the factor of two, it can be seen that one failure is added to the actual number of failed devices. This appears in the calculation as a failure occurring at the end of the test. This ensures that the test terminates with a failure, which is also a theoretical requirement, as well as allows a calculation of $\lambda$ even if no failure was observed.

\section{Temperature}

The failure rate of superconducting devices is inherently low. As result, the industry uses a technique called accelerated testing to asses the reliability of superconductors.

During accelerated tests, elevated stresses are used to produce, in a short period, the same failure mechanisms as would be observed under normal operation conditions.

One of the most important stress factors is the temperature. The ambient temperature is related to the junction temperature through the thermal resistance and power dissipation of the device. Devices can be tested close to their maximum junction temperature and then scale the resulting failure rate to lower temperatures. The scaling can be made using the Arrhenius equation:

$$
\lambda=A e^{\frac{\psi}{k T}}
$$

where $k$ is Boltzmann constant $\left(8.62 \cdot 10^{-5} \mathrm{eV} / \mathrm{K}\right), \mathrm{T}$ the temperature in degrees Kelvin and $\psi$ the activation energy. The failure rate at temperature $T_{1}, \lambda_{1}$, is related to the failure rate at temperature $T_{2}, \lambda_{2}$, by 


$$
\lambda_{1}=\lambda_{2} e^{\frac{\psi}{k}\left(T_{1}-T_{2}\right)}
$$

When enough data are given about the test condition and results, the manufacturer results will be used. Military handbook will be restricted to the components for which reliability data are not available and only the design technology is known. 


\section{Chapter 6}

\section{Validation and Failure Mode Analysis of the Quench Protection System}

\subsection{Component Validation}

The components used in the LHC Quench Protection System have been validated against radiation and, in some cases, also against ageing effects due to temperature and humidity.

\subsubsection{Radiation Tests on Electronic Equipment}

As has been already advanced in Chapter 3, the LHC tunnel will host most of the electronics needed by the accelerators. The closer to the machine the electronics can be placed, the better will be its performance (e.g. noise, remote diagnostic, etc.), and the lower the cabling needs.

\section{Radiation in the LHC}

Several studies have been developed [35] in order to estimate the radioactive characteristics of the LHC environment. They predict annual doses of $3.3 \mathrm{~Gy} / \mathrm{y}$ close to the arc magnets and $0.8 \mathrm{~Gy} / \mathrm{y}$ close to the tunnel wall alongside the magnets. This values come from the interactions of the beam with the residual gas, and the particle losses due to beam dynamics (i.e. particles outside the dynamical aperture of the beam are finally lost). Although the gas interaction losses are homogeneous along the arc, since they only depend on the vacuum properties, the collimation losses are more intense around the quadrupoles. Therefore, the electronics must be placed close to the dipole, preferably under the middle dipole. The dose also increases around a factor of 12 alongside the intermagnet gaps (see Fig. 6.1).

The dose in the long straight sections is lower due to better vacuum conditions, around $1 \mathrm{~Gy} / \mathrm{y}$. In contrast, the expected dose close to the high luminosity low- $\beta$ regions, which corresponds to $100 \mathrm{~m}$ before CMS and ATLAS experiments, is above $10^{3} \mathrm{~Gy} / \mathrm{y}$. The highest dose levels in the machine will reach $10^{4} \mathrm{~Gy} / \mathrm{y}$, but these will be restricted to a few hot-spot positions in the scraper regions.

Together with the effect of the integrated dose, the equipment in the tunnel will be exposed to Single Event Effects (SEE) (see Chapter 5), which will not depend on the received dose but on the particle and energy spectra. 


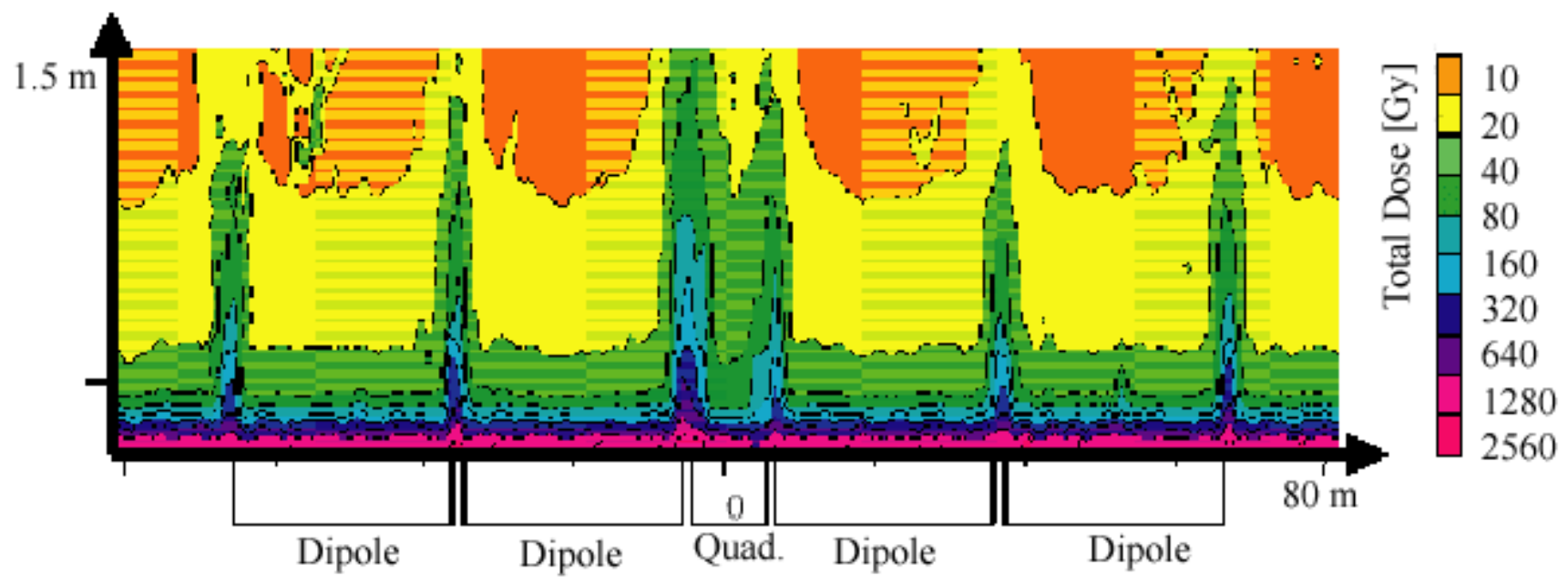

Figure 6.1: Simulated distribution along a regular cell of the total dose after 10 years of operation [34].

\begin{tabular}{lcc} 
Particle & Energy & Fluence $\left[\mathrm{cm}^{-2} \mathrm{~s}^{-1}\right]$ \\
\hline Neutrons & $<20 \mathrm{MeV}$ & $5.86 \cdot 10^{4}$ \\
& $>20 \mathrm{MeV}$ & $7.45 \cdot 10^{3}$ \\
Protons & $>20 \mathrm{MeV}$ & $3.38 \cdot 10^{2}$ \\
Charged Pions & $>20 \mathrm{MeV}$ & $5.87 \cdot 10^{2}$ \\
Photons & $>0.1 \mathrm{MeV}$ & $8.58 \cdot 10^{4}$ \\
\hline
\end{tabular}

Table 6.1: Particle spectra close to the LHC dipole surface [61].

The volume-averaged total fluences of the secondary particles induced by the beam losses in different regions of the LHC main dipole have been computed in [61]. The different particle fluences around the magnets can be then estimated. The results are listed in Table 6.1.

The dose in the LHC is mostly due to neutrons and photons. Hence, the neutrons will be the main responsible of damages on the LHC electronics. The presence of low and fast neutrons will be the cause of displacement damages mainly via nuclear reactions and elastic collisions. Although a large rate of high energy neutrons (above $5 \mathrm{MeV}$ ) is not expected, their presence will be enough to generate SEE, mainly via indirect ionisations caused by the products of the $(n, p)$ and $(n, \alpha)$ reactions.

In order to evaluate the feasibility of installing commercial electronics under these conditions, a test program have been carried out by the quench protection team since 1998 within the CERN Radiation Test Project [62].

\section{CERN Irradiation Facility TCC2}

A test facility has been set up in one experimental area of the LHC injector, the SPS (Super Proton Synchrotron), in order to validate the components planned to be used by the different LHC systems in the accelerator tunnel.

In the North Experimental Area, where the test zone is installed, three $400 \mathrm{GeV}$-proton beams hit three fixed targets. This generates three secondary beams, which downstream are separated in 


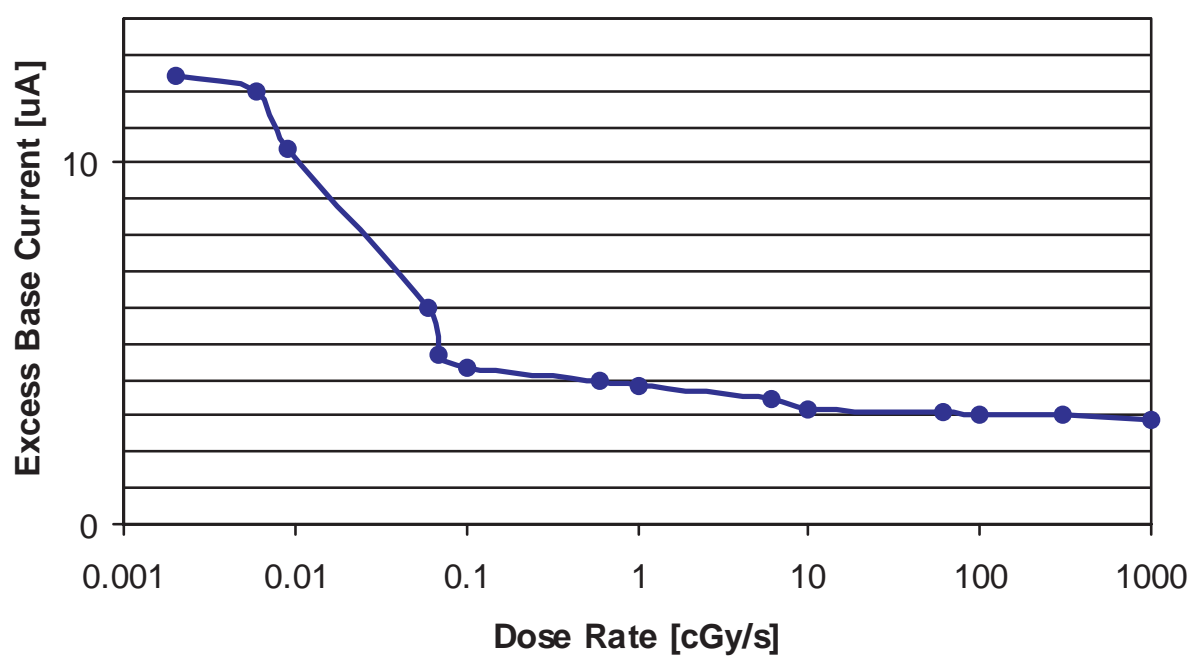

Figure 6.2: Excess of base current for a NPN discrete transistor against the dose rate after a 520 Gy irradiation [64].

six different lines. The irradiation facility is affected by two of these lines. The closest one is a high-intensity muon beam, which consists in an approximately 600 meters long pion $\left(\pi^{+}\right)$and kaon $\left(\kappa^{+}\right)$decay channel. The other carries a monoenergetic proton beam formed by the particles which did not interact with the fixed target [34].

The comparison between LHC and TCC2 radioactive environments are detailed in [63]. They conclude that for particle spectra above $E=1 \mathrm{MeV}$, the radiation environments of the TCC2 test area and LHC are the same. For computations including low energy neutrons, the fluence in TCC2 is higher than in the LHC. Thus, SEE problems experienced under the TCC 2 conditions are equal or greater than at the LHC. Regarding SEE, the TCC2 tests will provide a pessimistic result.

In order to evaluate the influence of the integrated dose rate at the LHC from TCC 2 test results, the important difference between dose rates has to be considered. While the dose rate expected in the LHC arcs (under the middle dipole) is in the order of $0.1 \mu G y / s$, in TCC2 it is about $50 \mu G y / s$.

A last factor must be taken into account to extrapolate the radiation results from TCC2 to LHC: the influence on the dose rate on the total integrated dose consequences. In the case of bipolar transistors, which are the most used by the QPS electronics, the gain degradation exhibit a strong dependence of the dose rate (enhanced low dose rate effect), the higher the dose rate, the smaller the degradation. The too accelerated test could not show properly the degradation that a device will have after receiving the same dose but with lower rate. The test would be too optimistic.

This effect is represented in Fig. 6.2 [64]. A simple NPN discrete transistor will suffer only a $25 \%$ of its true degradation for a $520 \mathrm{~Gy}$ exposure, at dose rates above $10 \mathrm{cGy} / \mathrm{s}$. The full TID response is not seen until dose rate is less than $0.01 \mathrm{cGy} / \mathrm{s}$. Since the dose in TCC2 is $0.005 \mathrm{cGy} / \mathrm{s}$, the effects due to the total integrated dose in the test zone can be considered to be the same than in the LHC tunnel.

Therefore, TCC2 is as a proper environment to carry out accelerated tests of the devices supposed to work in the LHC tunnel.

The devices under tests are controlled and monitored by a LabVIEW application from the control room or via the Internet. The devices are powered in order to evaluate their performance 
in real working conditions. The test results have been presented in different publications and workshops $[36,65,66]$.

\section{Irradiation Test Conclusions}

The irradiation tests on single components are already completed. The 2003 irradiation campaign will be focused on tests of full systems like quench detectors or data acquisition systems.

The four previous irradiation campaigns studied the performance of the components of the QPS subsystems which are supposed to work under radiation: the quench detectors, the quench heater power supplies and the cold diodes.

The results (see Appendix A) show that, in general, classical electronics based on bipolar technology (mainly used for the quench detectors) exhibit sufficient radiation tolerance for working in the LHC tunnel. The design of the electronic circuits using commercial components seems to be feasible. Despite this promising results, the tested devices can not be said to be totally insensitive to radiation. Almost all of them show a drift of the monitored parameters, which is directly correlated with the total received dose or the dose rate. Except for some voltage references, this drift is not important.

Not all the micro-controllers and fieldbuses tested worked under radiation. It was mainly due to the high sensitivity of elements using optical isolations to the displacement damage caused by the neutrons, together with the SEE provoked by the high energy particles.

Switch mode power supplies with $+5 \mathrm{~V}$ and $\pm 15 \mathrm{~V}$ used by the local quench detectors, showed different results depending on the manufacturer. In two thirds of the tested power supplies, the voltage rating of the MOSFET transistors were increased compared to the series model. After this enhancement, the best performing devices reached $800 \mathrm{~Gy}$ without failure.

The other tested devices are the aluminium electrolytic capacitors and the phase control thyristors used in the quench heater power supplies. As a consequence of the irradiation induced defects the leakage current of the capacitors increased. However, the absolute values stayed in a range that does not affect the proper performance of the power supply up to $200 \mathrm{~Gy}$.

A more severe problem is linked to the capability of firing the thyristors. All thyristors suffered from radiation induced defects, which affected their proper function after exposure to doses between 50 and $400 \mathrm{~Gy}$. Despite the problem with the thyristors no other radiation induced failures have been revealed by the tests.

Tests on different cold diode manufacturers reveal that difussed diode types can be regarded as radiation hard up to an integrated dose of about $1 \mathrm{kGy}$. They are suitable for protection in highly irradiated areas such as the arc quadrupoles. In contrast, the diffusion type diodes are much more sensitive to radiation and should be foreseen only to protect less irradiated elements such as the main dipole magnets [65].

The tests have demonstrated that devices with equal function but using different technologies may show a completely different performance under radiation. This is an important problem in the electronic market where parts become obsolete very quickly and the used technology can change from a batch to another. A commercial based design will be only possible if the technical specification, based on these results, are strictly followed during the series production.

\subsubsection{Stress Tests}

The two main components of the quench heater power supplies have been also tested under stressed conditions. The research has been made in collaboration with the Universitè de Lyon. 
The ageing of capacitors and thyristors has been monitored while they were exposed to its maximum working temperature and with different working cycles. Their performance showed up big differences between manufacturers. The results are shown in Appendix A.

\subsection{Protection Strategy Validation}

Although powerful simulation tools have been developed and used at CERN in order to assist during the design of the protection system (see Section 6.3), an experimental validation of the protection strategies and parameters is required before starting the series production of the system components.

Some experiments have been carried out in two CERN test stations in order to study the quench performance and magnetic field measurements at operating temperature of the different magnetic elements [30]. Nevertheless, the main drawback of the LHC quench protection is the series connection of huge inductance magnets. Hence, real experiments on the protection of a cell of magnets is needed in order to evaluate the QPS ability to protect the LHC superconducting circuits.

\subsubsection{The LHC Test String-2}

The String-2 is a full-size model of an LHC cell of the regular part of the arc. It is formed by six main dipole magnets with their sextupole and decapole spool piece correctors and two short straight sections, which host the lattice quadrupoles, and the orbit and lattice correctors. The first short straight section is connected to a prototype cryogenic distribution line running alongside the magnets. It distributes the helium at different temperatures and pressures (see Fig. 6.3).

This facility is the logical continuation of the String program, which started in 1997 with the String-1. It was composed by three 10-meter long dipole magnets (old magnet version) and one quadrupole magnet. The electrical system was very different from the current design (e.g. magnets and quadrupoles were powered by the same circuit) and only the main magnets were installed, nevertheless very useful experience was gained operating this string of magnets and many components were tested and optimised [67].

The String-2 was built to individually validate the LHC systems, to investigate their collective behaviour during normal operation and to evaluate the performance of the circuits and the protection systems after a quench.

The facility has been assembled and commissioned in two phases. The first phase included one half-cell with three dipoles and the two short straight sections. These were prototype magnets similar to the series magnets but heavily instrumented for the experimental programme.

The chain of magnets is terminated on the upstream end by the electrical feedbox (DFBS), which is a 6-meter long cryostat at $4.5 \mathrm{~K}$ and $135 \mathrm{kPa}$. It supports and cools $32 \mathrm{HTS}$ current leads and the so called lambda plate, which separates thermally and hydraulically its saturated liquid helium bath from the magnet pressurised superfluid helium bath at $1.9 \mathrm{~K}$ and $130 \mathrm{kPa}$. (see Fig. 6.4).

For the second phase, three pre-series dipole magnets were added thus completing a full LHC cell. The commissioning of the full String-2 started in May 2002, the experimental program will be completed in summer 2003. 


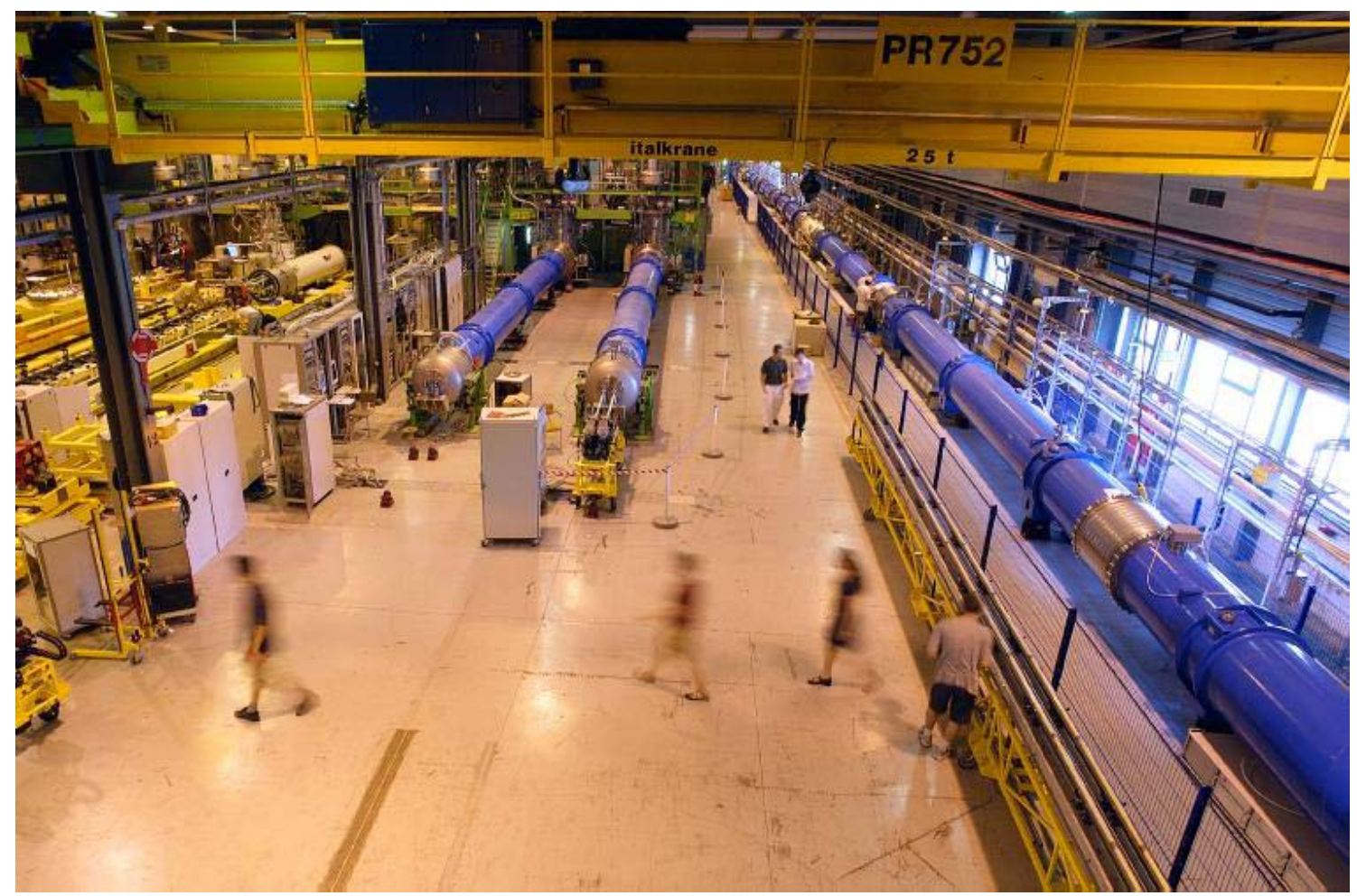

Figure 6.3: The LHC Test String- 2 assembled in the SM18 building at CERN. The two individual magnet test benches can be also seen.

\subsubsection{String-2 Quench Protection System}

The design of the quench protection for the String-2 is mainly based on the experience obtained from the first String. Its design was made before the QPS reliability studies started, hence it shows some differences with respect to the machine final design.

The dipoles and quadrupoles are protected by redundant quench detectors. Three quench signals are isolated and treated per detector. Two redundant voting systems formed by logic gates send the quench signal to the interlock system when at least 2 out of the 3 (2oo3) signals are above a $110 \mathrm{mV}$ threshold longer than $10 \mathrm{~ms}$. (see scheme $2003 \mathrm{~d}$ in Chapter 7).

The detection for corrector circuits and current leads is like explained in Chapter 3, with a single difference in the current leads: a temperature sensor has been mounted on each lead in order to provide a redundant detection. They are located in the interface between the resistive and the superconducting part and they will no be present in the machine version.

For the dipole circuit, one prototype of a $13 \mathrm{kA}$ energy extraction, facility, formed by four parallel branches of two switches connected in series, and one prototype resistor, has been installed. The sextupole chromaticity corrector circuits are protected with two 600 A energy extraction prototypes. They have been developed in two Russian institutes.

The quench heater power supplies protecting the dipole and quadrupole magnets have been made by the European industry.

The system includes an independent, distributed acquisition and monitoring facility, like the one planned to be installed in the LHC tunnel and underground areas. Dedicated processors send information concerning the status and other variables of the protection equipment to a supervision application. They also provide information about transients through local acquisition buffers where 


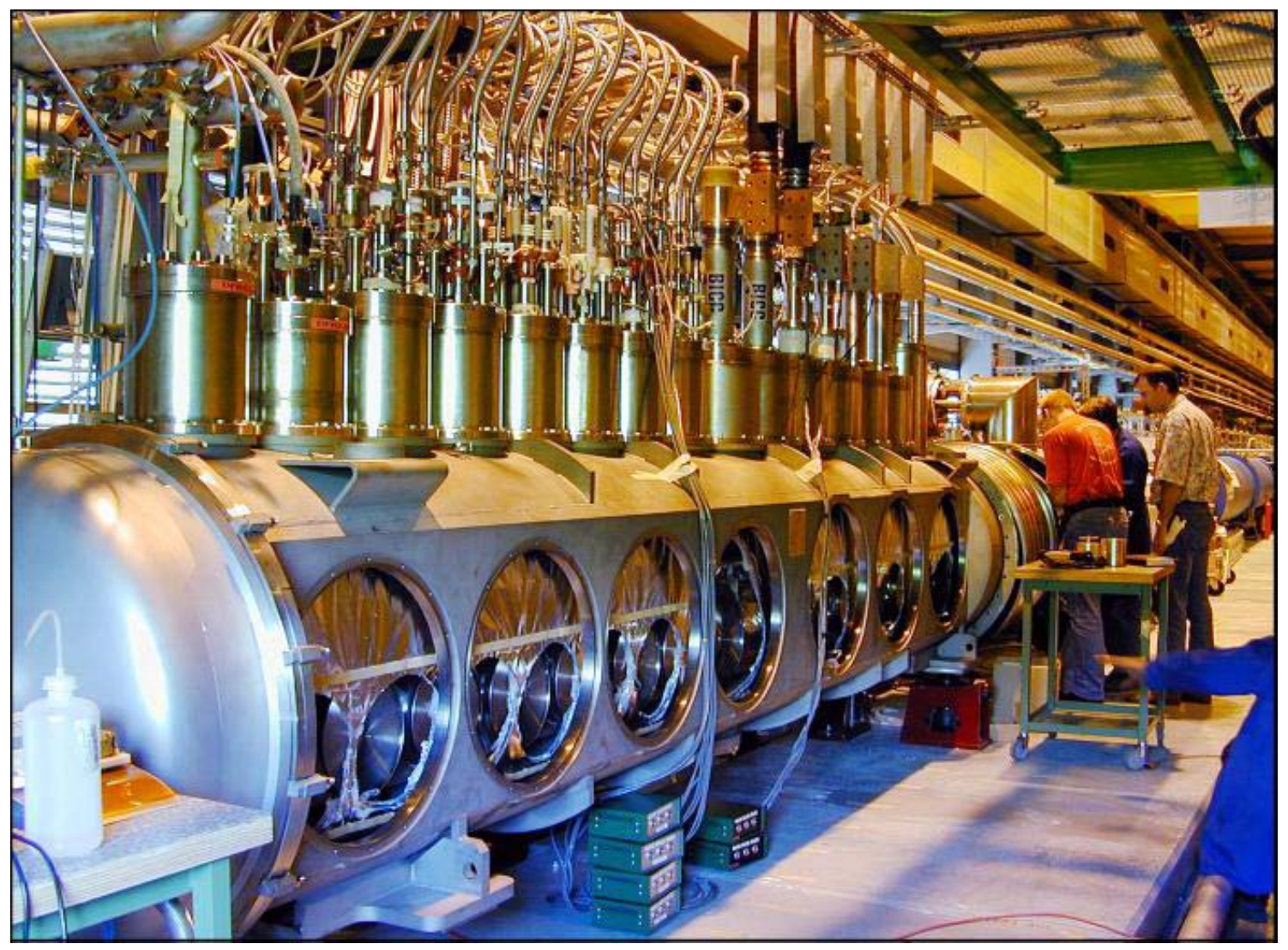

Figure 6.4: String-2 electrical feedbox with the 600 A and 13 kA HTS current leads mounted. 
the signals are recorded in case of a quench or other events.

About 600 signal channels are recorded. They include voltages, temperatures and signals from Hall probes (i.e. magnetic field sensors). The setting of the gain factors in the amplifiers is done by a computer running a LabVIEW application, which accesses the local signal conditioner via a field-bus.

\subsubsection{Commissioning and First Quenches in String-2}

Once the installation of the magnets and the subsystems was completed, the commissioning of the protection system was carried out in parallel with electrical, cryogenics, powering and vacuum checks. Before the first cool down, the integrity of the voltage taps was checked.

Following the cool down to nominal temperature and before powering the circuits, several verifications were carried out: insulation tests, transfer function measurements, coherence and integrity of the instrumentation, and tests of the cold by-pass diodes. The string circuits were commissioned independently and in gradual steps from low to nominal currents. At each step the protection system signals were verified. The first powering to nominal current of the dipole and the quadrupole circuits are shown in Fig.6.5.
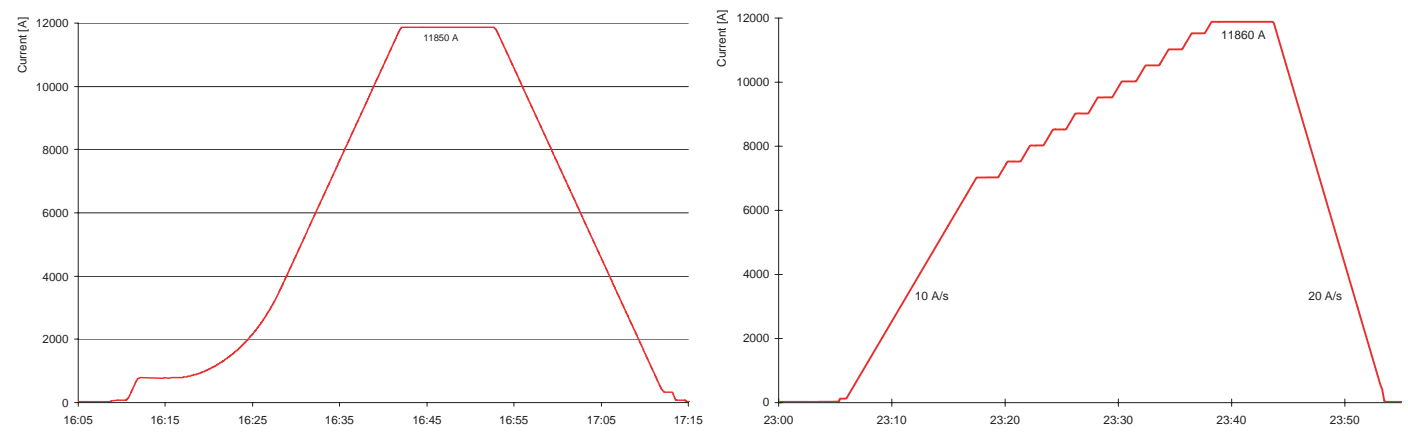

Figure 6.5: First powering of the diode (left) and the quadrupole (right) circuits of the String-2.

\section{Provoked Quenches}

The effectiveness of the quench protection heaters were measured at injection current and at intermediate level. The larger temperature margin at low field than at nominal field makes their effectiveness increase with current.

The original threshold for the local quench detectors was $200 \mathrm{mV}$. However, artificial symmetrical quenches (both apertures quenched at the same time by the quench heaters) at low current, show that this value was too high. The slower resistive voltage development across the apertures could compromise the integrity of the coil. It was decided to set the threshold for the LHC in $100 \mathrm{mV}$. The $110 \mathrm{mV}$ solution was the best approximation that could be implemented with the hardware installed in the String-2.

\section{Quenches in Dipoles}

Until the 2002 Christmas shutdown, the dipole circuit suffered 25 natural or provoked quenches. The protection system reacted properly and the magnets were safely sent to zero current. In Fig. 6.6 the quench signals and the circuit current after a dipole quench are plotted. 


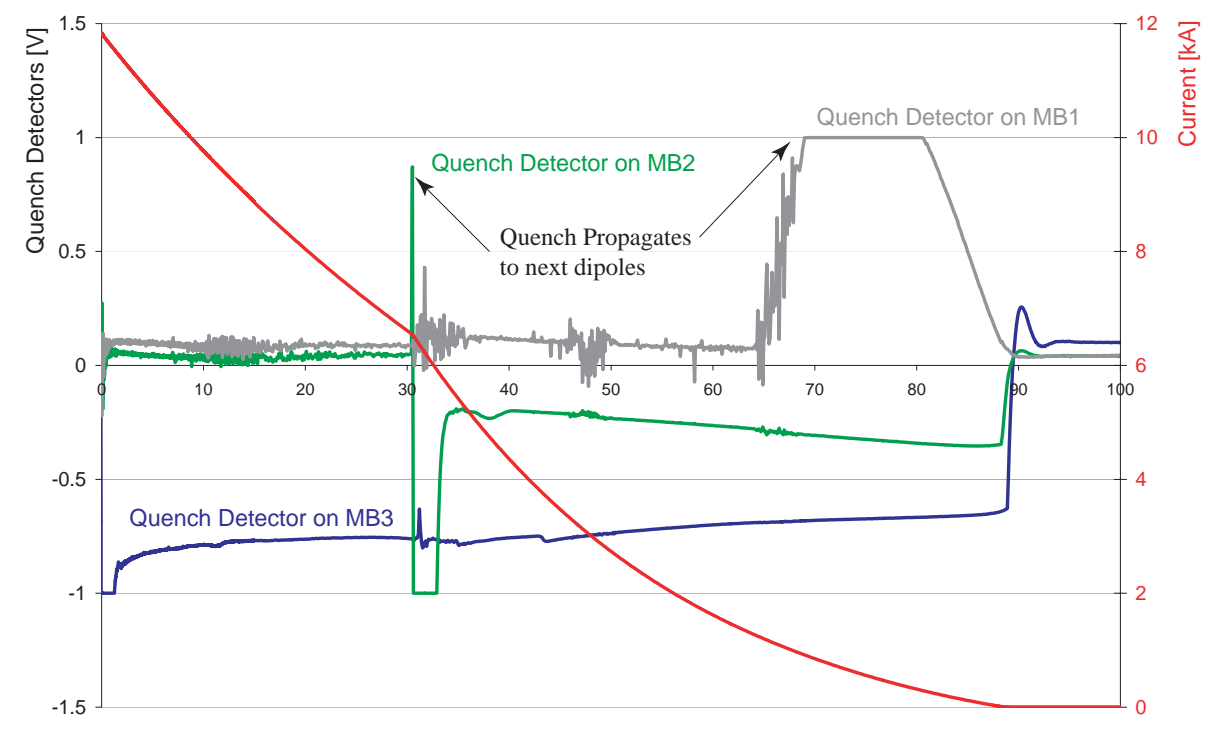

Figure 6.6: Provoked quench in a String-2 (first phase) dipole which propagates to the other two.

During the first phase, the quench always propagated to the neighbour magnet. When the quenched magnet was the middle dipole, the three dipoles quenched around half a minute later. The quench propagation was never observed to the quadrupoles since both circuits were never powered and quenched together.

In the second phase, some experiments have been done with release valves closed. This provoked the propagation of the dipole quench through the short straight section up to the next dipole. This was not observed in normal conditions, in which the quenches have been always confined in the half-cell where they have been originated. However, in the LHC the propagation of the quench through the short straight section in normal conditions is not excluded due to the higher volume downstream [68].

\section{Quenches in Quadrupoles}

The quadrupoles have suffered 15 quenches until December 2002. The quench protection system also reacted as expected and no propagation was observed to the other short straight section.

During String-2 run in 2003, tests of quench propagation from quadrupole to dipole are scheduled.

\subsection{Failure Mode Analysis}

The prototype String-2 has been a very powerful facility for better understanding the superconducting circuit behaviour and, which is even more important from the quench protection point of view, for getting experience in installation and commissioning of the system. However, the characteristics of the project do not allow to make failure tests, which are essential for evaluating the redundancy level of the components and the system hazards. 


\subsubsection{Quench Simulation Programs}

Two existing CERN tools have been used in order to verify the QPS behaviour in the LHC main dipole circuits: QUABER and SPQR.

\section{SPQR - Finite Difference Method}

A computer code has been developed at CERN (F. Sonnemann [18]) in order to simulate the different quench mechanisms in superconducting cables and magnets cooled by helium.

SPQR (Simulation Program for Quench Research) computes the material properties according to the geometry and input parameters (e.g. copper resistivity as a function of $R R R$ and applied magnetic field). Next, an initial Gaussian temperature profile is computed to provoke a quench with a certain peak temperature. The resistive propagation is computed taking into account heat generation, conduction, heat transfer through isolation to helium, forced quenching by heaters, and the impact of dynamic losses by magnetisation and induced eddy current (quench back).

\section{QUABER - Piecewise Modelling of Electrical Circuits}

The simulation package QUABER was developed at CERN (F. Rodriguez-Mateos et al. [69]) in order to simulate the voltage and temperature development of a series of superconducting magnets under different circuit configurations. It is based on the professional tool SABER, which allows the time transient simulation of any electrical or thermal circuit.

QUABER contains a thermodynamic model that defines the resistance development (quench propagation) as a function of time for different parts of a quenching magnet. QUABER has been used in this thesis for including the effect of protection strategies on the quenching circuit and evaluating the consequences that failures of the different components will have on the LHC dipole chains.

The results from QUABER depend on the input parameters that must be obtained from experiments or other simulation programs. SPQR has been used to set the quench parameters such as the quench propagation velocity.

\subsubsection{Validation and Calibration of the Models}

SPQR and QUABER have been validated and improved in parallel with the development of the LHC superconducting magnet program.

Although currently QUABER depends on the results given by SQPR, it was created first. The input parameters such as quench propagation velocity or the heater delay were obtained from experimental results. In this thesis, these simulation programs are used to study the behaviour of a whole chain of LHC superconducting magnets. Nevertheless the calibration of the model can only be carried out using single magnets or, at the most, a short chain such as the String 1 and 2 facilities explained above.

The quench tests carried out in LHC dipole prototypes show a calculated number of MIITS around $6 \%$ higher than the measured values. This difference is mainly due to the lack of relevant information about how the volumes underneath the heaters become resistive in the first instant. The model divides the circuit (and the coil) in blocks which are treated homogeneously (i.e. all the wires grouped by the block quench at the same time), this results in a slightly pessimistic result. It has been also demonstrated [70] that the simulation is able to trace back the voltage evolution, giving in general a good estimation of the peak values. It has been observed than the difference 


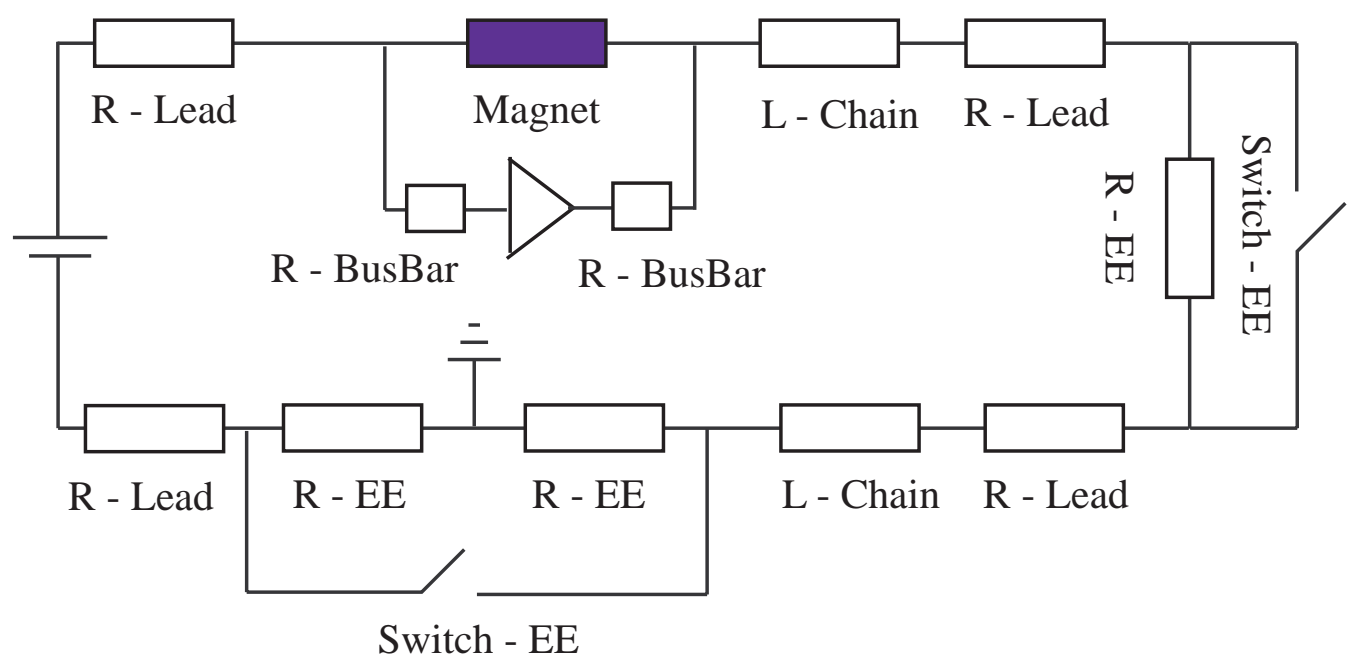

Figure 6.7: Simulated dipole circuit $\left(R_{\text {Lead }}=R_{\text {BusBar }}=20 \mu \Omega, \sum L_{\text {Chain }}=16.4322 H, \sum R_{E E}=\right.$ $150 m \Omega$ )

between the calculated values and the experimental results is larger when the quench resistances are triggered and the power converter is switched off in the beginning of the quench process. When the voltage reaches its peak value, the estimation is rather good.

Reliable QUABER results depend on the input quench parameters. The uncertainty of the input parameters is the dominating error source of the QUABER outcome. A limitation of the simulation model is due to the adiabatic assumption of the temperature evaluation. This sources of error have been solved with the development of SPQR.

The circuits and scenarios modelled in this thesis has been done using the two simulation programs together. This has given a higher confidence to the results, since more quench mechanisms are taken into account, and has made the model less dependent on experimental input data.

The calibration of SPQR has been done using the experimental data coming from the magnet prototypes and the first pre-series LHC magnets. A detailed description of this calibration together with a careful explanation of the thermohydraulic and electromagnetic models that the simulation program uses can be found in [18].

Once the quench data is obtained from SPQR, the elements of the electrical circuit can be treated as black boxes and QUABER is used to simulate the different failure scenarios.

\subsubsection{Simulated Circuit Model}

A superconducting magnet can be represented as an inductance in series with a resistance. In the superconducting state the resistance is zero, and starts increasing due to the temperature rise after a quench.

A SABER circuit has been designed in such a way for simulating the electrical behaviour of the LHC main dipole circuit. The quenched magnet has been divided into blocks connected in series. The different kind of blocks represent the inductances, quench heaters, interconnections and quench origin. The whole circuit is completed with the by-pass diode, switches and resistors for the energy extractor, and two pure inductances representing the rest of the magnets in superconducting state (see Fig. 6.7). 


\begin{tabular}{lcc} 
& Outer Layer & Inner Layer \\
\hline RRR & 180 & 150 \\
Ratio Cu/NbTi & 1.9 & 1.6 \\
Copper Area $\left[\mathrm{cm}^{2}\right]$ & 0.126083 & 0.153495 \\
NbTi Area $\left[\mathrm{cm}^{2}\right]$ & 0.066359 & 0.095934 \\
Cable Width $[\mathrm{cm}]$ & 0.173855 & 0.214005 \\
Metal Width $[\mathrm{cm}]$ & 0.127445 & 0.168533 \\
Cable Height $[\mathrm{cm}]$ & 1.54 & 1.51 \\
Metal Height $[\mathrm{cm}]$ & 1.51 & 1.48 \\
Self Inductance $[\mathrm{mH}]$ & 0.565 & 0.142 \\
\hline
\end{tabular}

Table 6.2: Cable parameters of the $13 \mathrm{kA}$ LHC dipole.

The maximum temperature of the conductor can be calculated with the heat balance equation that leads to the following expression:

$$
A_{C u} A \int_{T_{\text {bath }}}^{T} \frac{c(T)}{\rho_{C u}(B, T, R R R)} d T=\int_{t_{q}}^{t} I^{2} d t
$$

where $A_{C u}$ is the copper section of the conductor, $A$ the total conductor section, $c(T)$ the average specific heat (taking into account the superconductor and the copper) and $\rho_{C u}(B, T, R R R)$ the copper resistivity, which depends on the magnetic field, the temperature and the residual resistivity ratio. The right term of the equation is the quench load (see Section 2.3.4).

At the beginning of the simulation, QUABER computes two tables: the copper resistivity for temperatures from zero to $1000 \mathrm{~K}$ (using Devred Formulas [69]), and the quench load in MIIT for the same temperature range (using copper and superconductor heat capacity data). The values are computed for different magnetic fields.

At each time step, SABER calculates the electrical equations of the circuit. From SABER result of the current, QUABER computes the quench load and from it, the quenched volume, the new temperature map and the resistance. For each magnet block, the field distribution is computed using another computing code developed at CERN: ROXIE [71]. It calculates the peak field at a given current and the average field for every block. Finally QUABER gives the map temperature in the magnet and the different layer voltages.

The parameters used by the model can be divided in two classes: the data related of the electrical characteristics of the circuit (e.g. inductance, cable geometry, RRR, etc.) and the values related to the quench protection (e.g. detection and switches delay, quench heater discharge, quench velocity, etc). The main cable parameters used in this study are listed in Table 6.2 for the inner and the outer layers.

The parameters of each model block can be modified by the user in order to simulate different failure scenarios (e.g. delay of detection, switch failure, broken diode, etc.). The nominal parameters used for the quench performance and protection are listed in Table 6.3.

\subsubsection{Nominal Operation}

The protection delays used in the model are summarised in Figure 6.8. These values do not have to be necesarily in the drawn order. 


\begin{tabular}{l|c} 
Quench Velocity & $1500 \mathrm{~cm} \mathrm{~s}^{-1}$ \\
Quench Prop. between Cables & $20 \mathrm{~m} \mathrm{~s}^{-1}$ \\
Quench Prop. between Layers & $20 \mathrm{~m} \mathrm{~s}^{-1}$ \\
Initial Current & $11796 \mathrm{kA}$ \\
Quench Detection Threshold & $100 \mathrm{mV}$ \\
Quench Validation Time & $10 \mathrm{~ms}$ \\
Switches Delay (from detection) & $20 \mathrm{~ms}$ \\
Power Abort Delay (from detection) & $5 \mathrm{~ms}$ \\
Diode Turn-On Voltage & $8.3 \mathrm{~V}$
\end{tabular}

Table 6.3: Quench and protection performance parameters of QUABER dipole model.

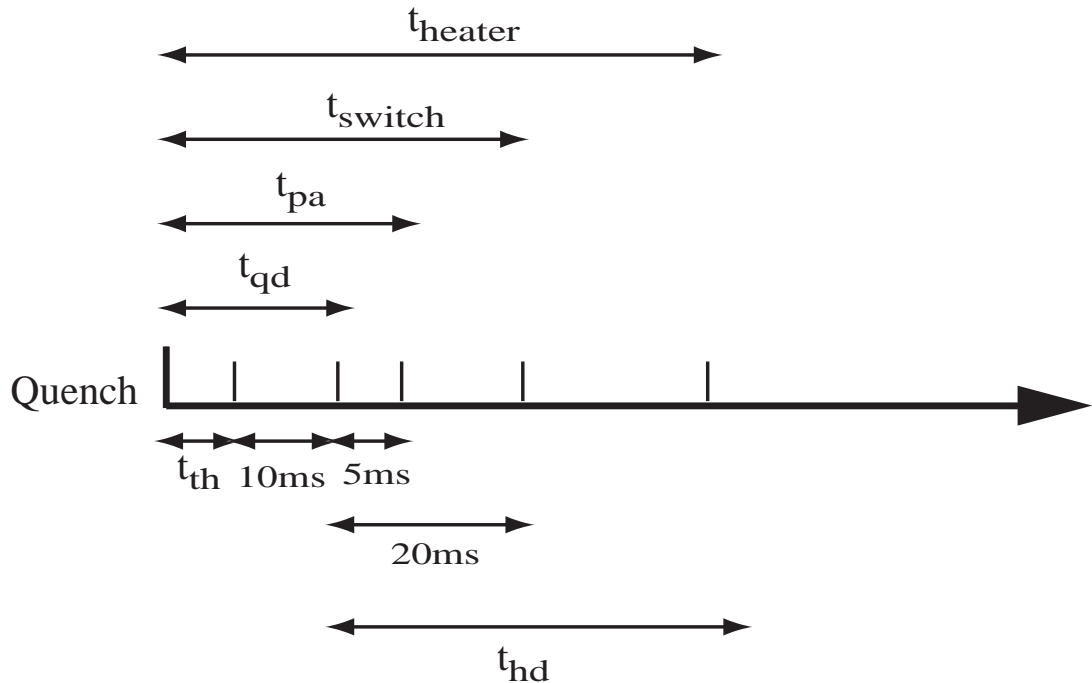

Figure 6.8: Simulation times $\left(t_{t h}\right.$ :time to reach detection threshold, $t_{h d}$ : delay from heater quench since detection, $t_{q d}$ :total detection time, $t_{p a}$ : time to power abort, $t_{\text {switch }}$ : time to open the switches, $t_{\text {heater }}$ : heater quench delay from quench occurrence).

The QPS is triggered when the difference between the voltages across each aperture stays above the detection threshold longer than the validation time. The total delay from quench occurrence is given by the time to reach the detection level, $t_{t h}$, plus the validation, which in this case is $10 \mathrm{~ms}$.

In order to compute this first value, a quench is simulated in the magnet with the protection system disabled. The voltage across the magnet reaches $100 \mathrm{mV}$ after $15.6 \mathrm{~ms}$. Taking into account the validation time, the protection system will be triggered $25.6 \mathrm{~ms}$ after the quench $\left(t_{q d}=25.6 \mathrm{~ms}\right)$.

It was demonstrated in [72] that this value depends on the origin of the quench. If the quench starts in a low field region of the coil, it will propagate slower than in a high field zone. Nevertheless, quenches in low field regions are very unlike, hence through this study only high field quenches have been considered.

The detection time decreases for conductors with lower RRR. Since 180 and 150 are quite high values of RRR in LHC dipoles, the study should be conservative enough. However, at low 
temperatures, a lower RRR means an higher resistivity (see Section 2.3.3). This causes a faster resistance development and current decay, resulting in lower temperatures. The differences are, however, negligible, hence the values above have been used during the study.

The effectiveness of the heaters will be defined in the model by $t_{h d}$, which corresponds to the time needed by the quench heater to warm up the coil after the quench has been detected (see Fig. 6.8). This value will depend on the magnetic field of the region where the heater is placed: the heated block and the magnet current. The heater strips are located around the magnet coils like shown in Figure 3.7. Experimental results in [30] gave the following formulae for $t_{h d}$ in high and low field heaters:

$$
\begin{gathered}
\text { HF Heaters : } t_{h d}=0.27 e^{-0.185 I_{0}} \\
\text { LF Heaters : } t_{h d}=0.27 e^{-0.14 I_{0}}
\end{gathered}
$$

Being $I_{0}$ the magnet current expressed in [kA]. Using the values listed in Table 6.3, a quench at full current $(11,796 \mathrm{kA})$, detected at $100 \mathrm{mV}$ will have the delay times listed in Table 6.4.

$$
\begin{array}{l|cl|c}
t_{t h} & 15.6 \mathrm{~ms} & t_{p a} & 30.6 \mathrm{~ms} \\
t_{q d} & 25.6 \mathrm{~ms} & t_{\text {switch }} & 45.6 \mathrm{~ms} \\
t_{\text {hd }} \mathrm{HF} & 30.45 \mathrm{~ms} & t_{\text {heater } \mathrm{HF}} & 56.05 \mathrm{~ms} \\
t_{\text {hd }} \mathrm{LF} & 51.78 \mathrm{~ms} & t_{\text {heater } \mathrm{LF}} & 77.38 \mathrm{~ms}
\end{array}
$$

Table 6.4: Delay times for a full current dipole (see Fig. 6.8).

The Figure 6.9 shows the magnet voltage, currents, quench load, and hot spot temperatures for a quenching dipole with the nominal values listed above.

In the next sections, the circuit behaviour will be simulated when the parameters above change due to failures in the protection system.

\subsubsection{Diode Failure}

Three kind of failure modes can be observed in the by-pass diodes: open circuit, shorted diode and turn-on delay. These failures may have different causes.

- Many Quenched Magnets in an Arc: If all the heaters in an octant (or most of them) were misfired, one or several diodes of the last quenching magnets would be destroyed due to high reverse voltage.

In order to avoid this, the QPS will not allow simultaneously firing of quench heater power supplies in all the octant. Nevertheless, a large number of magnets can be quenched by the beam (e.g. quadrupole circuit failure, or local poor vacuum conditions). In this case the magnets remaining superconductor will see a high reverse voltage and their diodes be destroyed. 


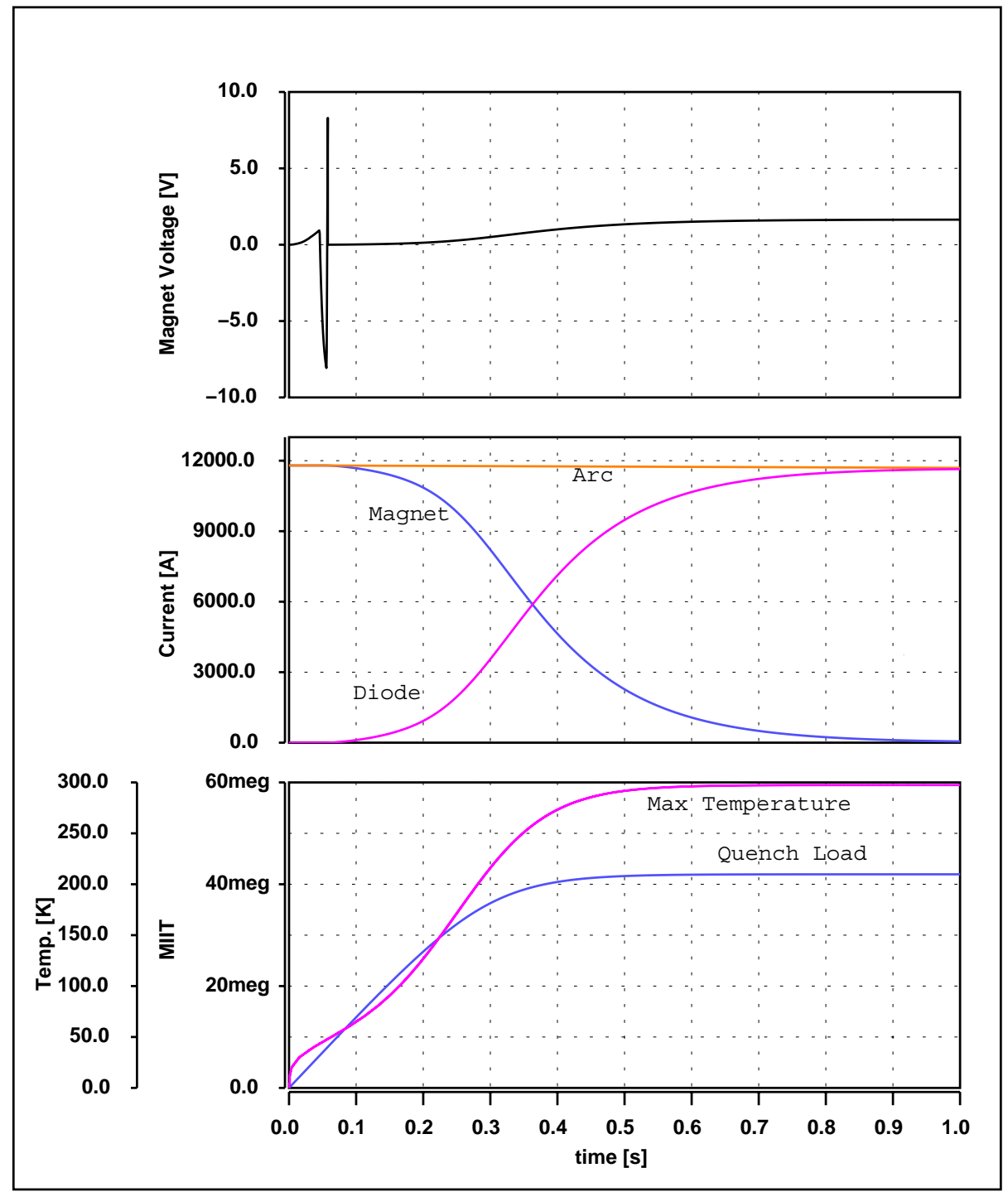

Figure 6.9: QUABER results for a properly protected dipole circuit. 
- Failure of the Energy Extraction Switches: Although this failure probability is low (see Chapter 9), if it happens, the diode will not be able to carry the current for the long decay time of the circuit without the extraction resistors. The diode melting itself would not be a problem to keep the quenched magnet integrity since the high pressure will preserve the conduction. However, the diode bus bars will have to stand a much larger energy dissipation than in normal conditions, which can lead to an open circuit due to its melting.

- Radiation: The turn-on voltage of the diodes increases with radiation. Despite the radiation tests carried out, the risk of this is high, since the dose received by the diodes is much higher than for the other QPS elements. On the other hands, this kind of defect should be rapidly detected and corrected by thermal annealing.

- Maintenance Mistake: Checks will be carried out frequently in the diodes in order to evaluate their degradation due to radiation. Although its risk must be minimised by defining a proper inspection policy, this failure may occur.

- Catastrophic Beam Loss: Although it is extremely unlike, there exists the possibility of suffering a full beam loss in the diode area, which would destroy the diode and quench the magnet at the same time.

\section{Open Diode}

If the diode or its bus bar are in open circuit, the detection of the quench will not be affected. The dipole will not be isolated from the rest of the circuit and the coil will have to stand the current during the whole decay time (if not melted before). Figure 6.10 represents the evolution of current, voltage and quench load of the quenched magnets. Their evolution under normal conditions (i.e. diode working properly) has been also plotted with dashed lines.

The quench load above 120 MIITS implies a hot spot temperature around $4000 \mathrm{~K}$ that will melt the conductor. Moreover, the magnet voltage will reach $190 \mathrm{kV}$. It implies a voltage across the magnet chain in the order of $100 \mathrm{kV}$ that can also damage the diodes of the non quenched magnets due to the high reverse voltage.

The magnet current, which will be the same as the arc current (until the conductor melting), will decrease faster than in normal conditions since the total circuit resistance is higher.

\section{Turn-On Delay}

The turn-on voltage of the diode can be increased mainly due to the irradiation.

As can be seen in Fig. 6.11, the hot spot temperature do not become dangerous until the turn-on voltage reaches some kilovolts. It has been also represented the voltage across half arc (quench is always assumed in the middle magnet) for different turn-on voltages. The circuit can be said to be safe with a delay up to $1 \mathrm{kV}$.

\section{Short-circuited Diode}

If the dipole is short-circuited through the diode, the voltage development after the quench will be slower, thus the detection time will be increased. This modifies the whole protection performance. 


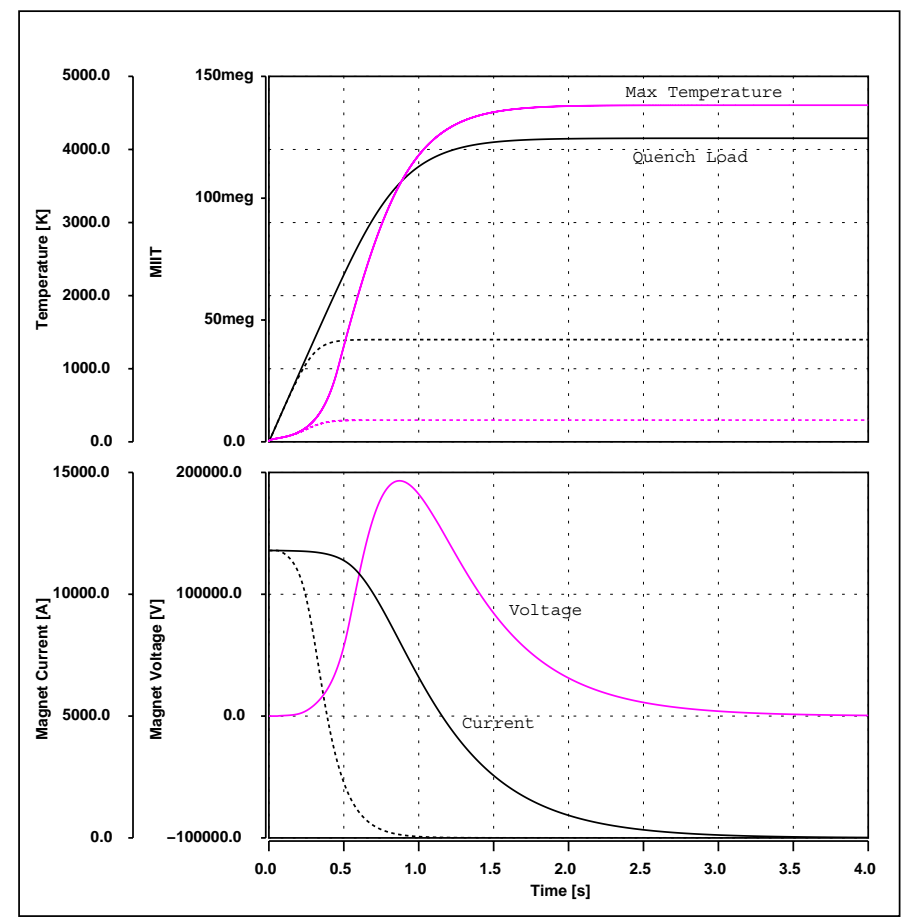

Figure 6.10: Up: Maximum temperature (light) and quench load (dark) for diode in open circuit; Down: magnet voltage (light) and current (dark); Nominal values are represented by dashed lines.

Within nominal current the quench signal will reach the detection threshold in $621.2 \mathrm{~ms}$. This delay of more than half a second will be enough to generate a hot spot temperature around $2000 \mathrm{~K}$ (80 MIITS) that will compromise the integrity of the conductor (see Fig. 6.12).

The magnet current will have an slower delay and the cell current will evolve with the same time decay but, of curse, it will start later. The voltage will not be affected.

\subsubsection{Energy Extraction Failure}

The main objective of the energy extraction is to avoid overheating of the by-pass diode busbars. The energy dissipated in the busbar in a time $t$ after a quench is given by the equation

$$
E_{b b}=\int_{0}^{\infty} R_{b b} I(t)^{2}
$$

With $R_{b b}$ being the busbar resistance (copper) and $I(t)$ the current. Equation 6.4 can be expressed in terms of current decay constant:

$$
E_{b b}=R_{b b} I_{0}^{2} \int_{0}^{\infty} e^{-\frac{2 t}{\tau}}=R_{b b} I_{0}^{2} \frac{\tau}{2}
$$

where $I_{0}=11796 A$ and $\tau$ the current decay constant. As can be seen in Fig. 6.7 the energy is extracted by two equal and independent resistors each one of $75 \mathrm{~m} \Omega$.

The redundancy of the switches is deeply analysed in Chapter 9. Here, only the full failure of the extractor bank is considered. This means that the switches are unable to open the circuit and lead the current to the extraction resistors. 


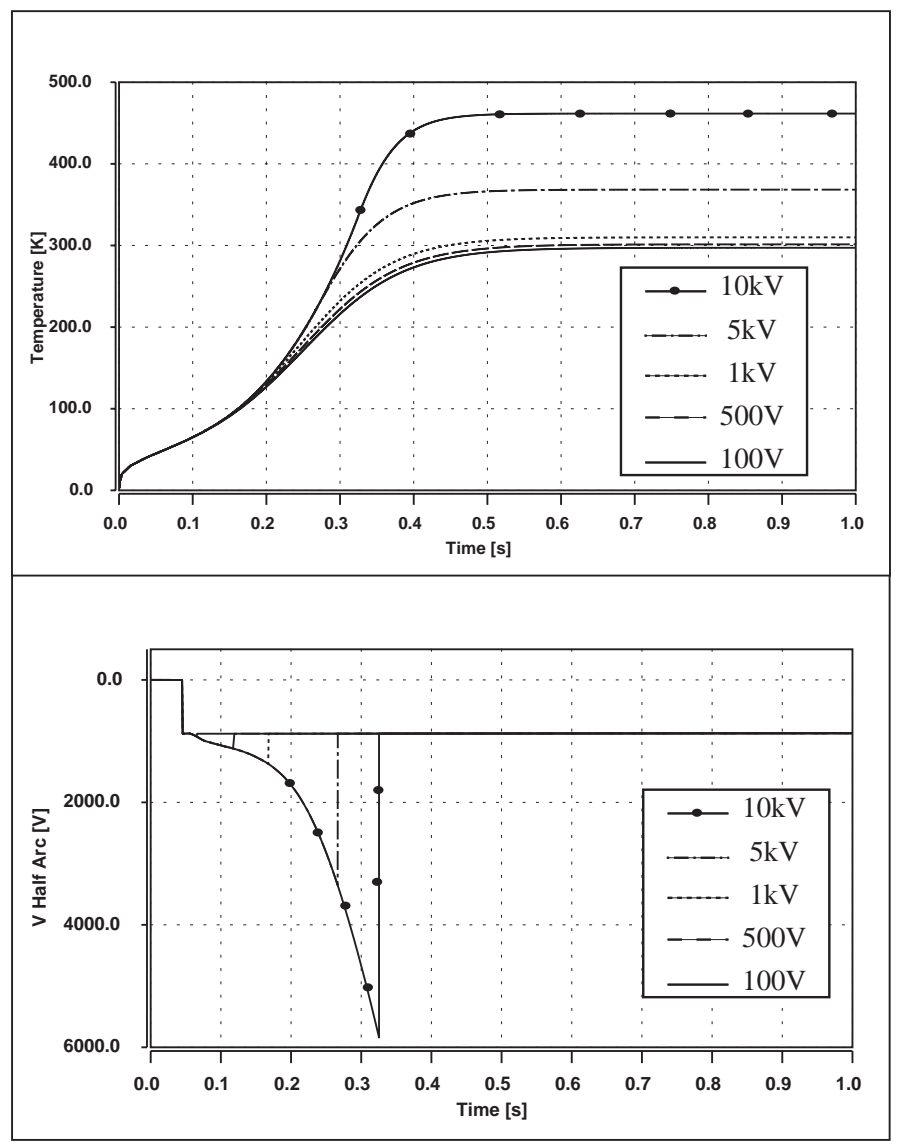

Figure 6.11: Up: Hot spot temperature and voltage along half cell for different turn-on voltages of the cold diode.

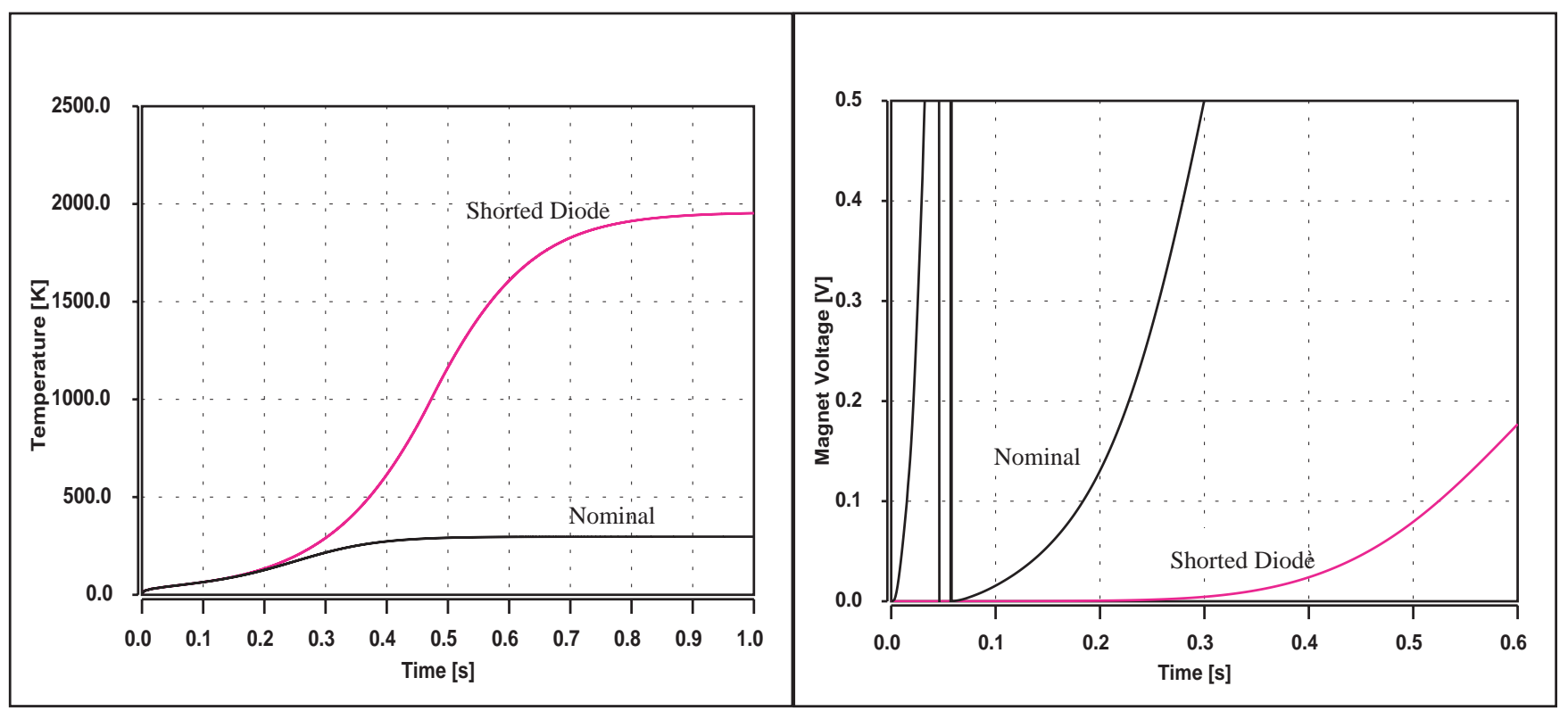

Figure 6.12: Hot spot temperature (left) and magnet voltage (right) in nominal condition (dark) and shortcircuited diode (light). 


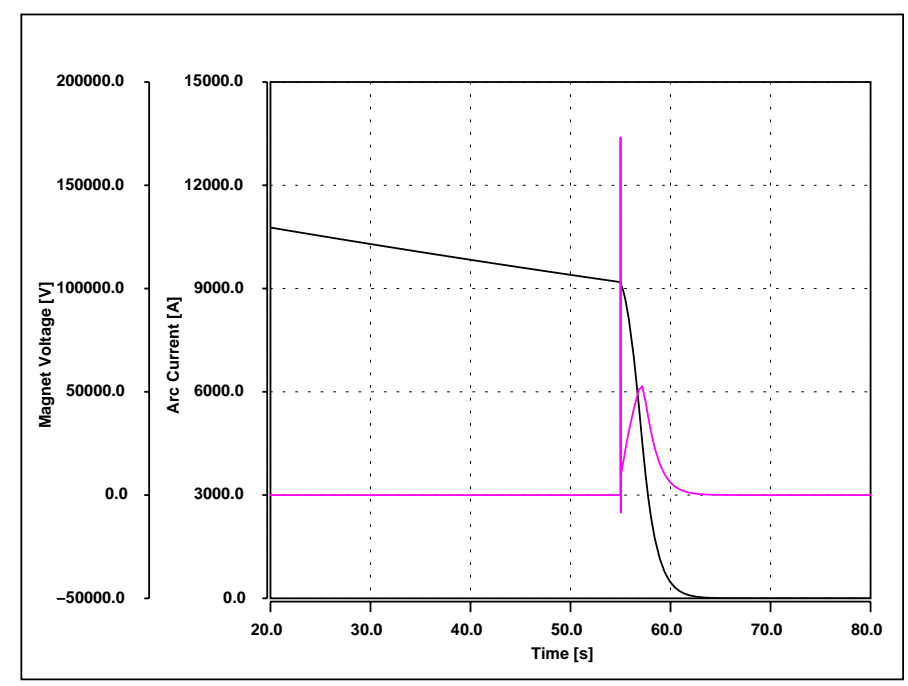

Figure 6.13: Magnet voltage (light) and arc current (dark) after the failure of one energy extraction and diode damage due to the slow current decay.

Table 6.5 lists the current decay constant and the total energy dissipated by the busbars $\left(R_{b b}=40 \mu \Omega\right)$ for the two possible failures (i.e. one or both switch banks failed) and the nominal performance.

\begin{tabular}{lccc} 
& $R_{\text {total }}$ & $\tau$ & $E_{b b}$ \\
\hline Nominal & $150.14 \mathrm{~m} \Omega$ & $109.4 \mathrm{~s}$ & $304.5 \mathrm{~kJ}$ \\
One Switch Failed & $75.14 \mathrm{~m} \Omega$ & $218.7 \mathrm{~s}$ & $608.6 \mathrm{~kJ}$ \\
Two Switches Failed & $140 \mu \Omega$ & $117,372 \mathrm{~s}$ & $0.327 \mathrm{GJ}$ \\
\hline
\end{tabular}

Table 6.5: Failures in the energy extraction banks: total resistance of the circuit $R_{\text {total }}$, current decay time constant $\tau$ and energy dissipated in the diode busbars $E_{b b}$.

The diode busbar are not designed to dissipate $600 \mathrm{~kJ}$, thus the failure of one of the switch banks will cause an irreversible damage in the magnet, although the diode itself will survive due to the safe design of the heat sinks.

This is one of the most dangerous failures in the machine. Two solutions have been studied in order to face switch opening failures. Firstly, firing of several quench heaters along the circuit in order to get enough resistance and, secondly, installation of a high current fuse in series with the switches. These solutions are developed in Chapter 9.

The other circuit parameters such as voltages and quench load are not dangerously affected by the slower discharge until the diode busbar melts. The generated voltages after the diode fails have been simulated under the pessimistic assumption that the busbar is burned once the dissipated energy is equal to the one in nominal conditions (i.e. both energy extractors properly working).

The dramatic effect of one energy extraction bank failure on the magnet voltage and cell current is represented in Fig. 6.13. The temperature reaches a hot spot above $1000 \mathrm{~K}$. 


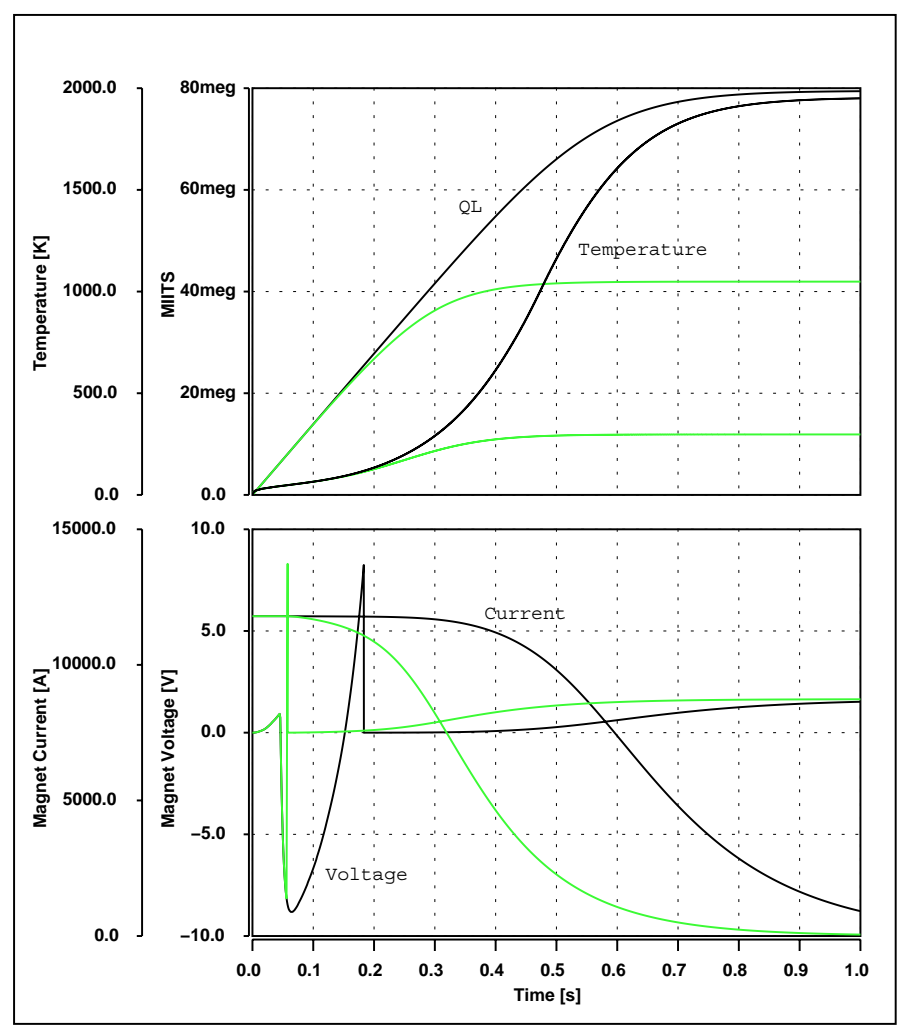

Figure 6.14: Magnet performance with full quench heater failure. The nominal values are represented by lighter lines.

\subsubsection{Quench Heater Supply Failure}

Each pair of high field and low field quench heaters can be powered independently (see Fig. 3.7). The redundancy level and their usefulness have been simulated.

\section{Full Heater Failure}

In Fig. 6.14 the effect of a full heating failure (i.e. no quench heater power supply discharged) is represented. The absence of forced quench cause a slower increase in the magnet voltage and a consequent delay in the diode turning on. This delay of only few tenths of second is enough to raise the hot spot temperature above $1500 \mathrm{~K}$.

\section{Field Influence}

Together with the low and high field heaters, the possibility of using quench heaters between the coil layers has been considered (inner layer heaters).

In Fig. 6.15 the quench load have been plotted for different combinations of high field (HF), low field (HF), and inner layer (IL) heaters. It can be seen that high field heaters are enough to protect the magnet, whereas low field heaters are unable to protect the magnet on their own. The effect of the inner layer heaters is very small. Hence, only high field heaters will be used in the final design, however the design keeps the possibility of including low field heaters in order to increase the redundancy. 


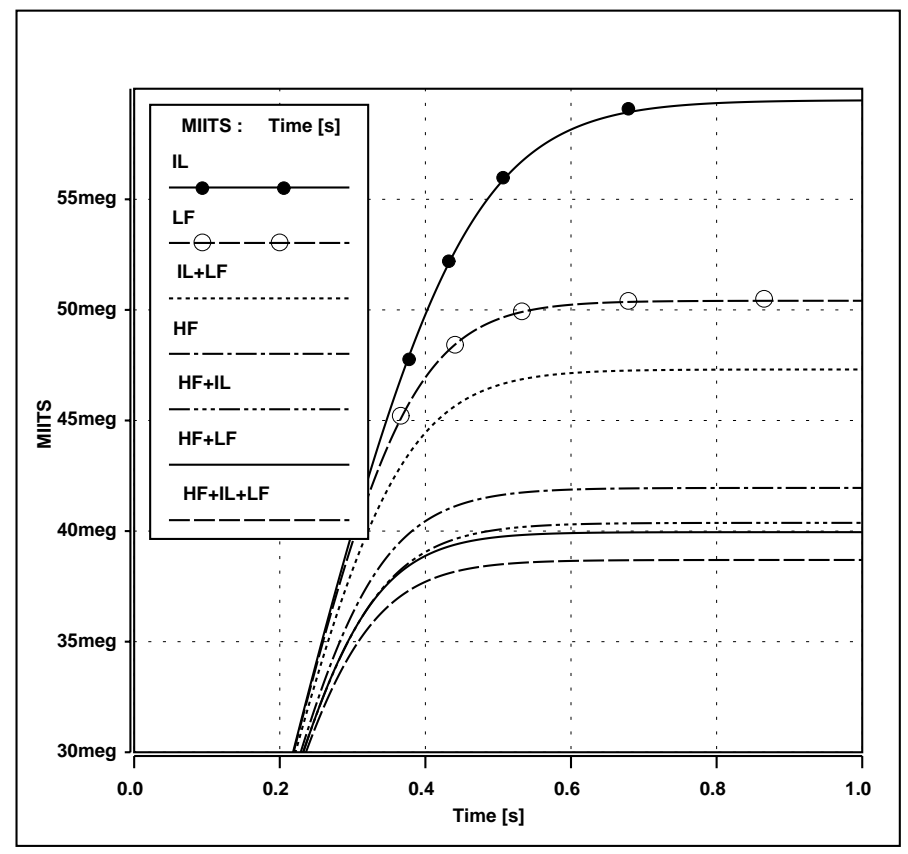

Figure 6.15: Quench load for different heater combinations (HF: high field, LF: low field, IL: inner layer).

\section{HF Heater Redundancy}

The plots explained above assume a full discharge on the energised heater strips. Simulations have been carried out in order to evaluate the impact on the magnet of problems in the quench heater power supplies. In Fig. 6.16 the effect of an ineffective discharge of one or several HF quench heater pairs (i.e. the energy given to the coil is not enough to initiate the quench around the heater strip) has been represented.

It can be observed that the effect will be slightly different if the failed heater is covering the area in which the natural quench started (dashed line). The necessity of the artificial quench propagation is lower close to the quench origin.

The hot spot temperatures reached when one quench heater power supply fails are not dramatically high and can be stand by the coil. However, a failure of two or more power supplies will compromise the integrity of the coil.

\section{Heater Delay}

The last failure considered is the delay of the artificial quench generation. This will cause a later turn on of the cold diode. The effect of this delay on the quench load is represented in Fig. 6.17.

A delay in the heater discharge below $100 \mathrm{~ms}$ can be tolerated by the coil. Above this value the hazard of damaging the conductor is too high.

\subsubsection{Power Abort Failure}

The $13 \mathrm{kA}$ power converters are provided with a protecting diode, which avoids voltages above $16 \mathrm{~V}$.

After the quench is detected a power abort signal is sent via the global interlock to the power converters. In case there is a problem in the signal generation or transmission, the converter will 


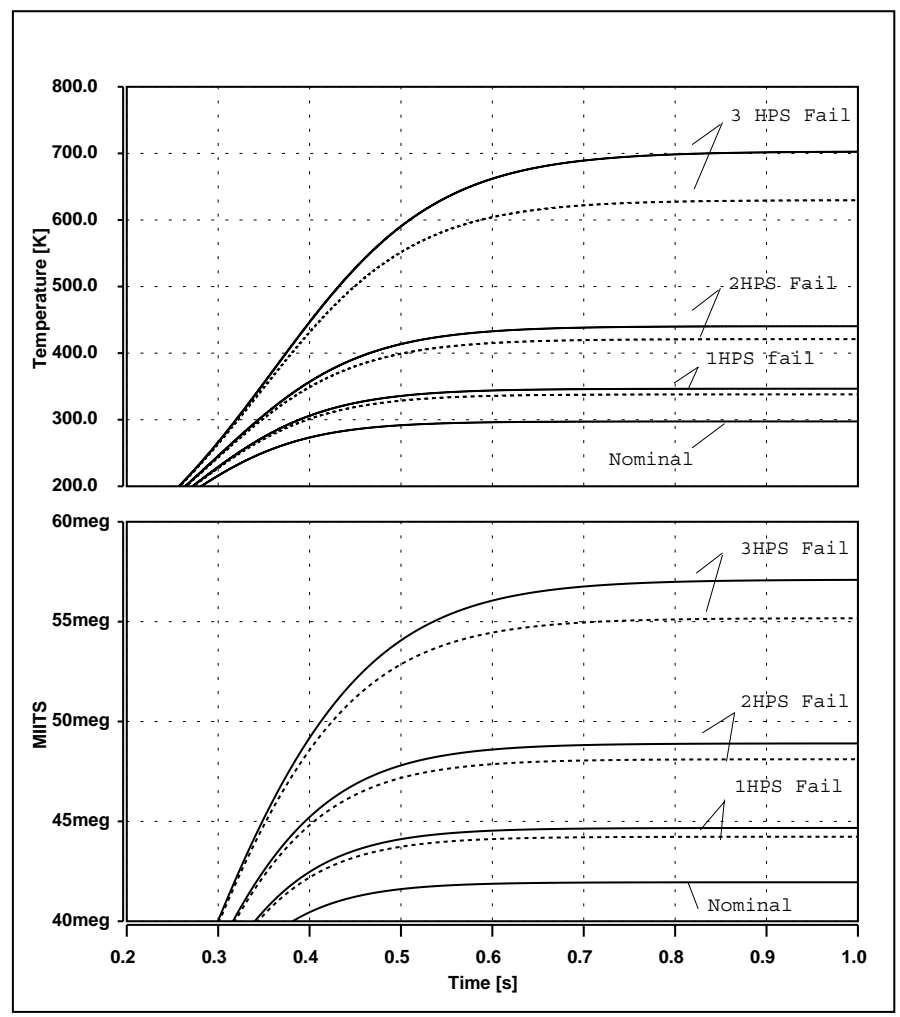

Figure 6.16: Quench load and hot spot temperature after the failure of one, two, or three quench heater power supplies in a main dipole. Dashed lines: quench origin below failed heater.

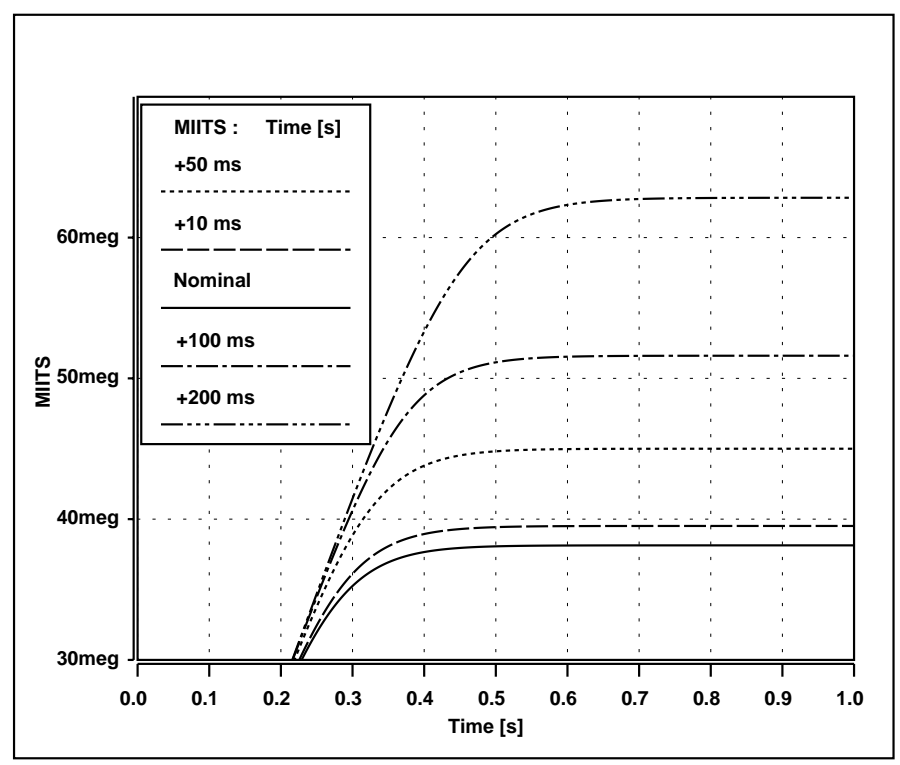

Figure 6.17: Quench load for different delays of the high field heater discharge from the nominal performance. 


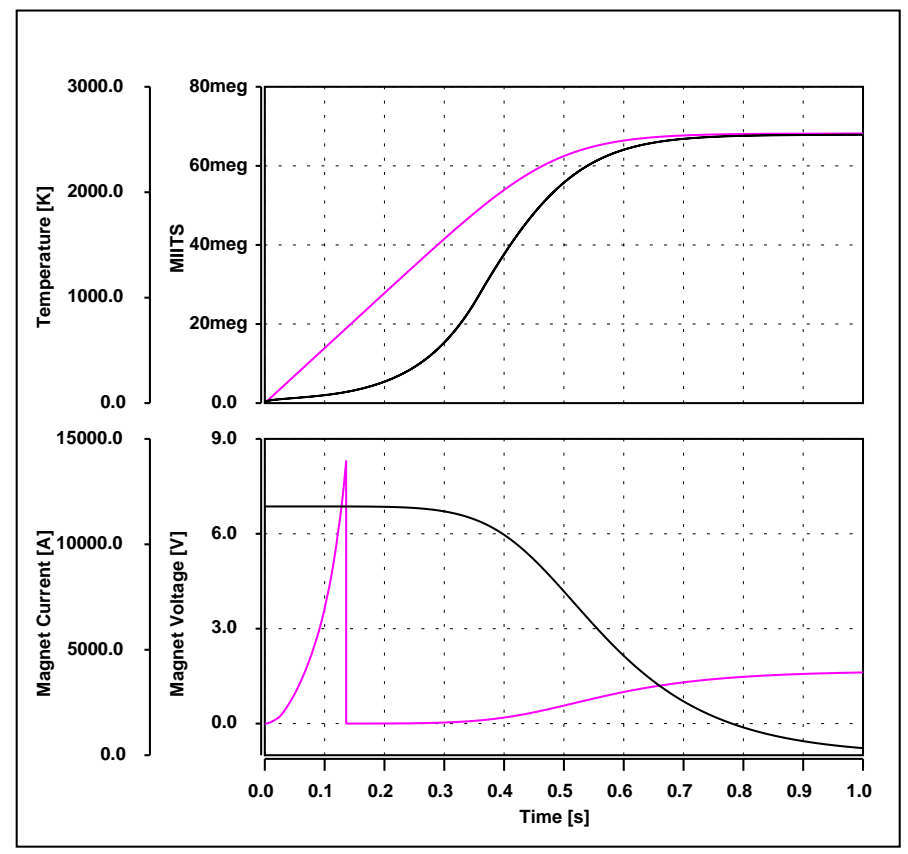

Figure 6.18: Magnet performance after missed quench. Up: quench load (light) and hot spot temperature (dark); Down: magnet voltage (light) and current (dark).

see the voltage of the magnet plus the voltage across the energy extractors. It will reach $16 \mathrm{~V}$ almost simultaneously with the switch opening, hence the power abort failure will be transparent to the rest of the circuit.

\subsubsection{Quench Detector Failure}

This failure has an impact in all the other elements of the system, since all the active protection is triggered by the quench detectors.

\section{Missed Quench}

If a quench is not seen by the detector, the quench will slowly propagate until the global quench detector, whose mission is the detection of transitions in the busbars, sees the voltage across the whole circuit. Nevertheless, the detection will be far too late and the coil will be already damaged.

A failure in the quench detection can be considered as the combination of a failure in all the active elements (i.e. quench heaters, energy extraction switches and power abort) together with a delay in the passive element (i.e. cold diode).

Fig. 6.18 shows the magnet parameters after a quench detection failure. Although the quench load will be enough to damage the conductor, it can be noticed that the effect will not be worse than it was in the case of failures in the diode. This shows the importance of the cold diode performance within the quench protection system. The failure in the passive element has worse consequences than a simultaneous failure of all the active components. 


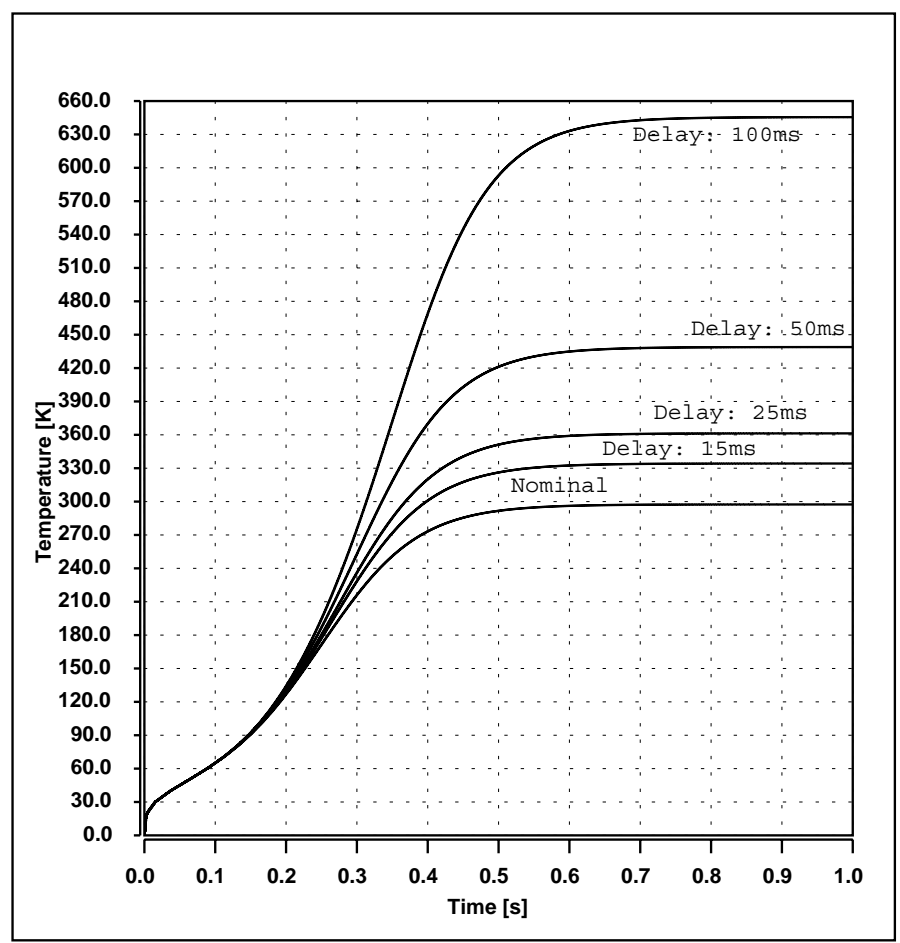

Figure 6.19: Hot spot temperature for different detection delays in a main dipole.

\section{Delayed Detection}

The second possible failure from the local quench detectors is a detection delay. This will retard all the protection strategy. This temporary malfunctioning will not have any dramatic effect as long as the delay is below 50-60 ms. Longer delays will compromise the integrity of the conductor and the isolation (see Fig. 6.19).

\subsubsection{Fault Tree Analysis (FTA)}

The results of this sections are summarised by the fist levels of the Fault Tree Analysis for the $13 \mathrm{kA}$ magnets quench protection system, which are represented in Fig. 6.21 (damaged magnet top event) and Fig. 6.20 (false quench top event). 


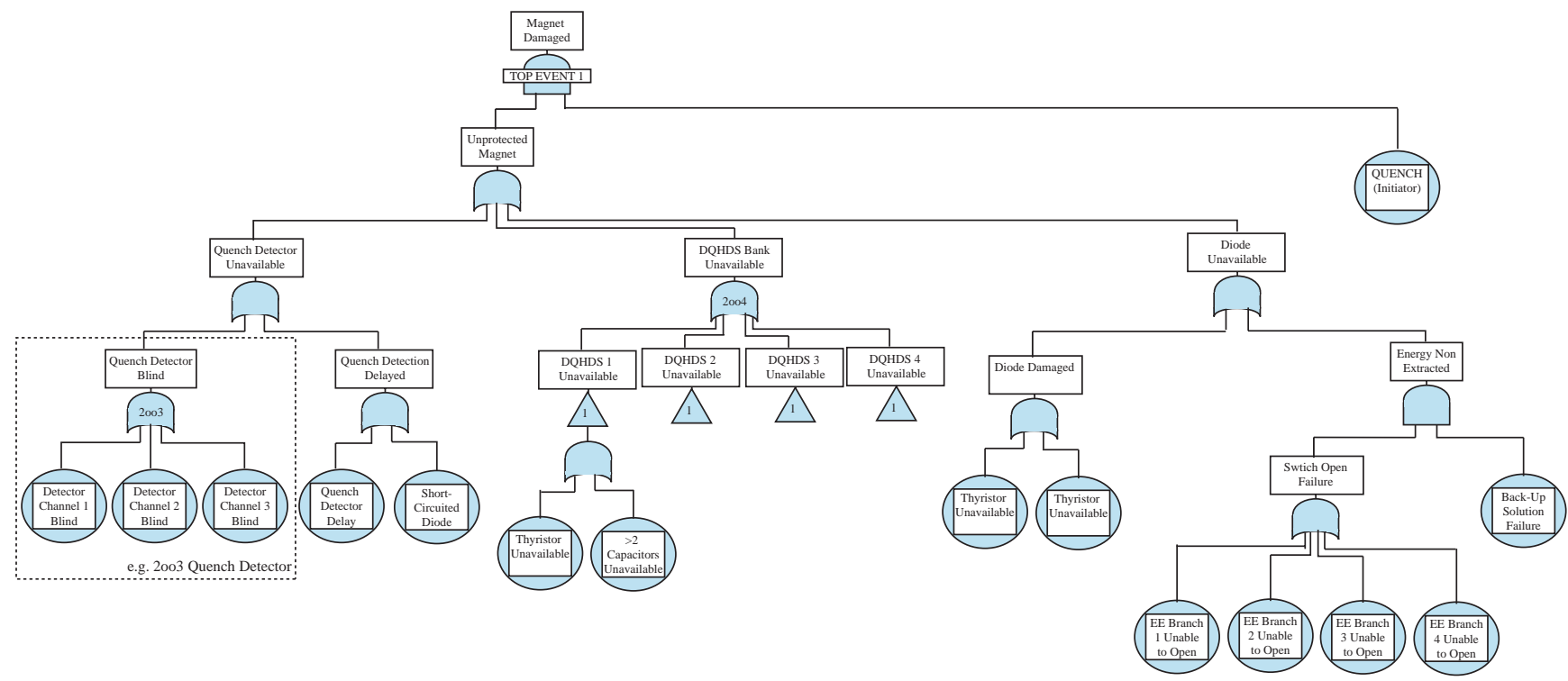

Figure 6.20: Fault tree analysis (FTA) for the quench protection system of the LHC $13 \mathrm{kA}$ main magnets: Main Failure.

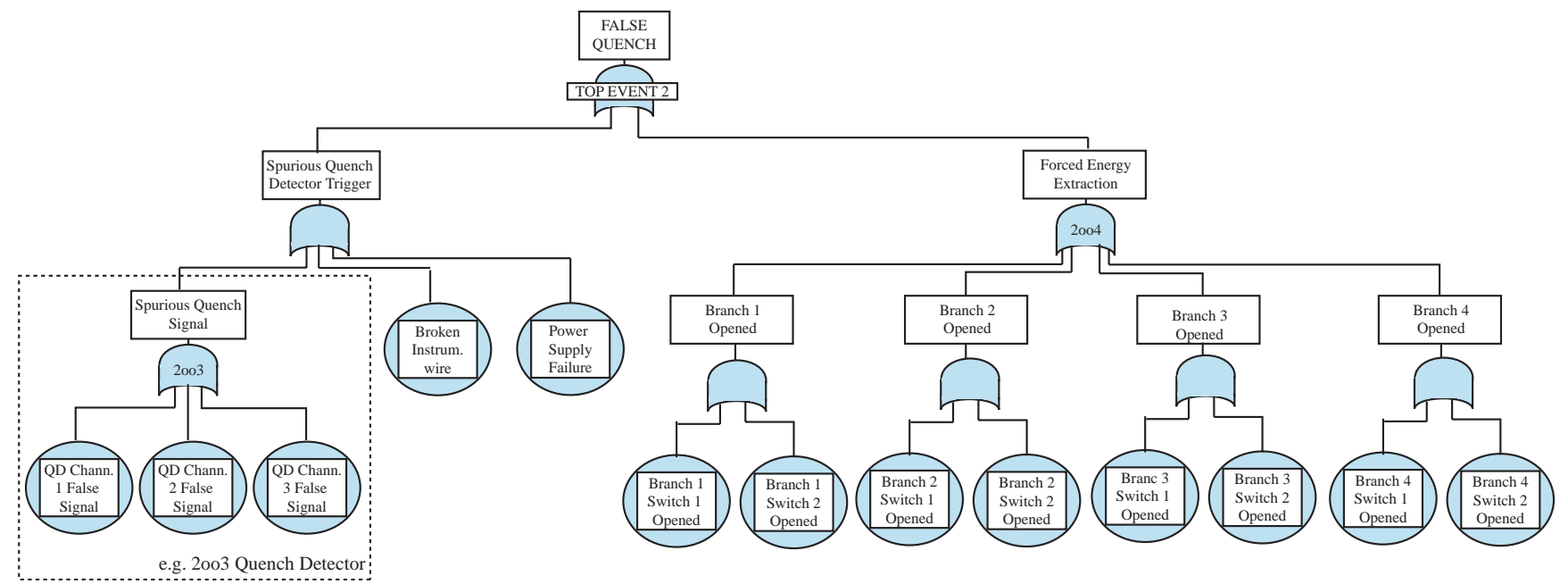

Figure 6.21: Fault tree analysis (FTA) for the quench protection system of the LHC $13 \mathrm{kA}$ main magnets: False Quench. 



\section{Chapter 7}

\section{Reliability of the LHC Quench Detectors}

\subsection{Main Magnet Local Quench Detectors (DQQDL)}

The lattice quadrupoles and main dipoles of the LHC will be continuously monitored by 2016 quench detectors. The reliability of such detectors has an important impact on the total LHC reliability. A non-detected quench can easily provoke an irreversible damage (see Chapter 6) on the quenched element and an important loss of time and money for its removal or substitution. On the other hand, too safe designs could often generate false quench signals decreasing also the total available time of the machine.

\subsubsection{Detector Design}

Quenches in the main dipoles are detected by comparing the voltages of the two apertures. When a quench appears, a floating bridge, formed by the two magnet apertures and two balancing resistors, becomes unbalanced and the quench signal is generated. The principle is the same for the quadrupoles but, since the apertures are powered by different circuits (focusing and defocusing), the comparison is made between voltages of two poles.

The floating bridge detection has been adopted because the detector must be able to see the quench at any stage of the accelerator powering cycle, hence it must distinguish the inductive from the resistance voltage.

The quench detector must provide galvanic isolation between input and output as well as to ground according to the required withstand levels [73]. All isolating components of the quench detector (not only the local but all families) as the power supply, digital isolators (pulse transformers) and relays exhibit voltage withstand levels of $2.5 \mathrm{kV}$.

The voltages are routed out of the cryomass by two redundant instrumentation wires. One instrumentation amplifier, a voltage reference and a comparator complete the generation of the quench signal. In order to increase its reliability, the quench detector is based on a multichannel evaluation. A quench is detected when $k$ out of $n(k-o o-n$ or $k o o n)$ signals stay above the threshold $\left(V_{t h}=100 \mathrm{mV}\right)$ longer than a certain time period $\left(t_{v a l}=10 \mathrm{~ms}\right)$.

The general layout of a quench detector can be, therefore, subdivided in several common functional blocks (see Fig. 7.1): instrumentation wires from the cold-mass to the quench detector racks, redundant quench signal generators (amplification, buffering and comparator), multichannel evaluation logic, time discriminator and powering.

In addition, the detector is equipped with a small data acquisition system, which provides information about the voltage across the magnet apertures (or poles in the case of the quadrupoles), 


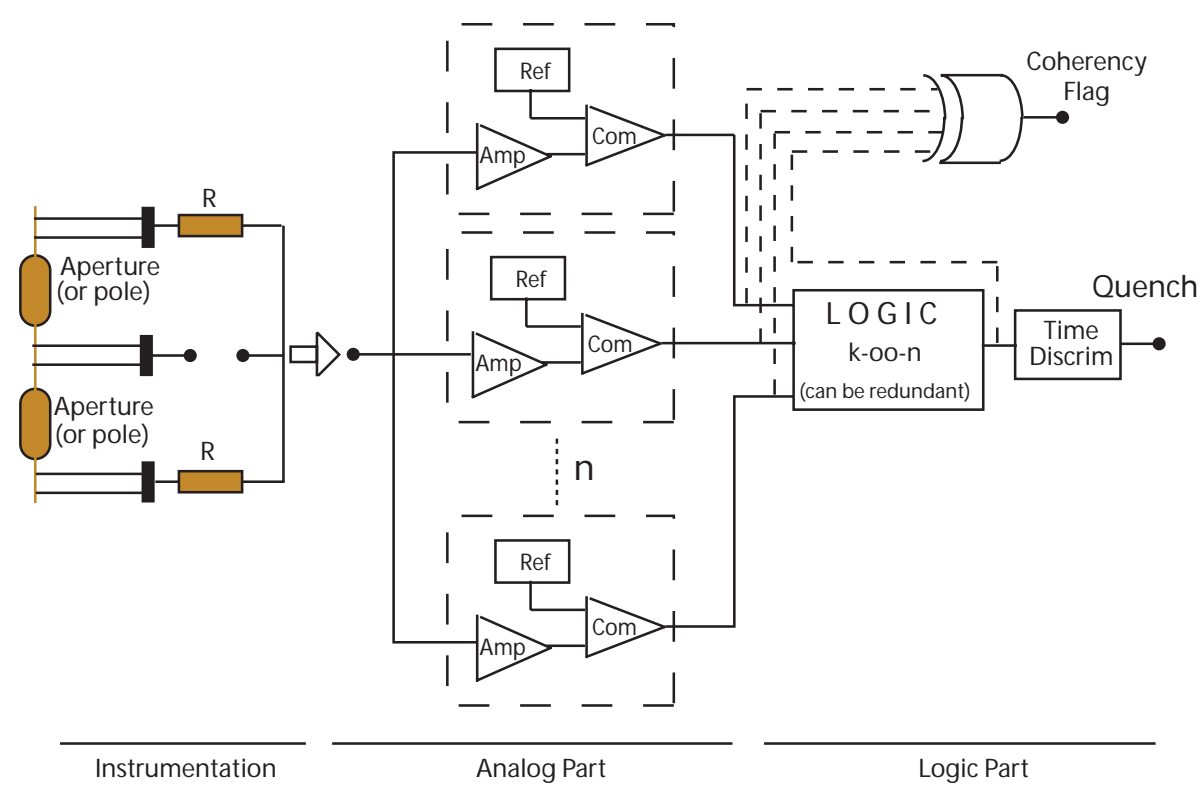

Figure 7.1: General DQQDL layout with short-circuited wires and instrumented for checks.

the output of the bridge, and a number of status flags used by the global interlock and system maintenance. A circular buffer is used. In case of a quench its content will be frozen.

The quench detector information is provided to the machine protection system and to the accelerator supervision system (i.e. global interlock). All the information relevant for the accelerator supervision system, post mortem analysis, test model enabling and power permit will be transmitted via fieldbusses and ethernet links. The quench status signal, which changes to low any time a quench occurs, will be transmitted via hardwired links to the machine protection system. The link is done via a current loop.

These detectors are located in racks under the main dipoles inside the LHC tunnel. These racks house also the quench heater power supplies. Due to radiation issues explained in Chapter 6, the racks will be installed as far as possible from the short straight sections.

\subsubsection{Failure Modes}

The local quench detectors, like the other detector families, show three different failure modes:

- False Quench (FQ): Due to the fail safe design (negative logic), this is a safe failure mainly generated by powering trips, broken instrumentation wires or problems in the voltage references. It has an expected cost of 5-hour downtime [55].

- Unprotected Magnet (UM): This dangerous failure occurs when a detector becomes blind and is unable to perform its basic function. Although this is a highly undesirable situation it has no actual cost.

- Missed Quench (MQ): This is the main failure. The superconducting element quenches when the detector is blind. 


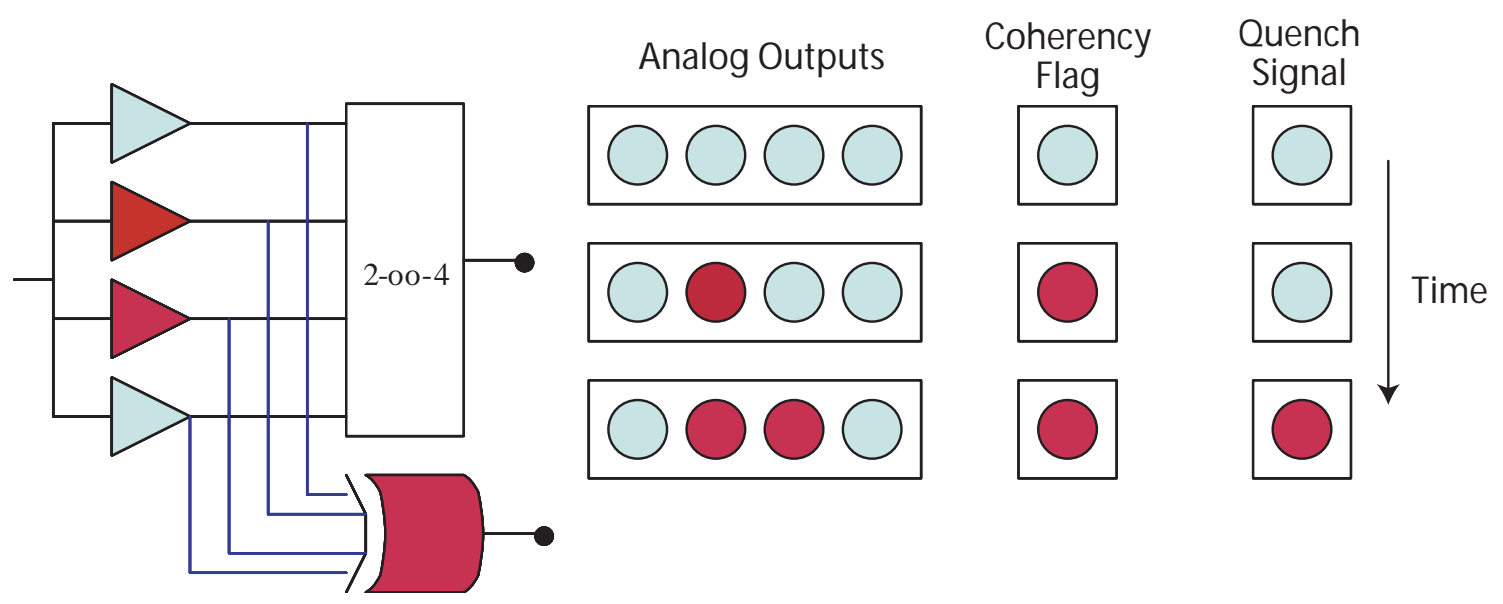

Figure 7.2: Coherency check in a 2004 detector. Up: No failure, middle: one channel in Quench state, down: false quench generation.

\subsubsection{Maintainability}

The maintainability of the quench protection system must adapt itself to the LHC operation schedule. The machine will run during 20 years with a yearly long shutdown during the wintertime, usually ranging from November to March.

After commissioning, during routine operation of the collider, filling is expected to take place once or twice per day followed by a physics coast from 10 to 21 hours. A daily technical stop of about one hour is foreseen to allow minor maintenance of the equipment and instrumentation. Unless specifically requests (see QT below), this technical stop will keep the current converters at stand-by current in order to preserve the magnetic history of the machine. In order to increase the availability of the detection system, two different checks are foreseen:

- Coherency Test (CT): The redundant channels and the quench signal are connected to a XOR gate (see Fig 7.1) that will send a warning (coherency flag) to the control room when any of the signals is different from the others. This increases the reliability against false quenches (see Fig. 7.2).

Since this flag is only advising about a potential false quench in the future, no action will be taken until the next Power Abort. Although the coherency flag is continuously monitored, repairs can be postponed for several LHC runs in order to reduce the tunnel access needs. The repairs for preventing false quenches will be carried out with a certain frequency, named in the model as the coherency test frequency.

- Quench Test (QT): A simulated quench signal is sent from the control room setting all the channels at Quench level. If one or more do not change, the coherency flag will provoke the generation of a warning message (see Fig. 7.3). If all the channels are blind, the output of the quench detector will show the failure staying at No Quench state. If the check is followed by a repair of the damaged element the availability of the system is set to 1 (as good as new). Since this test implies the discharge of the quench heater power supplies, it can only be performed when the magnets are not powered.

Figure 7.4 represents a part of the system Markov diagrams used to implement the structure function matrix. 


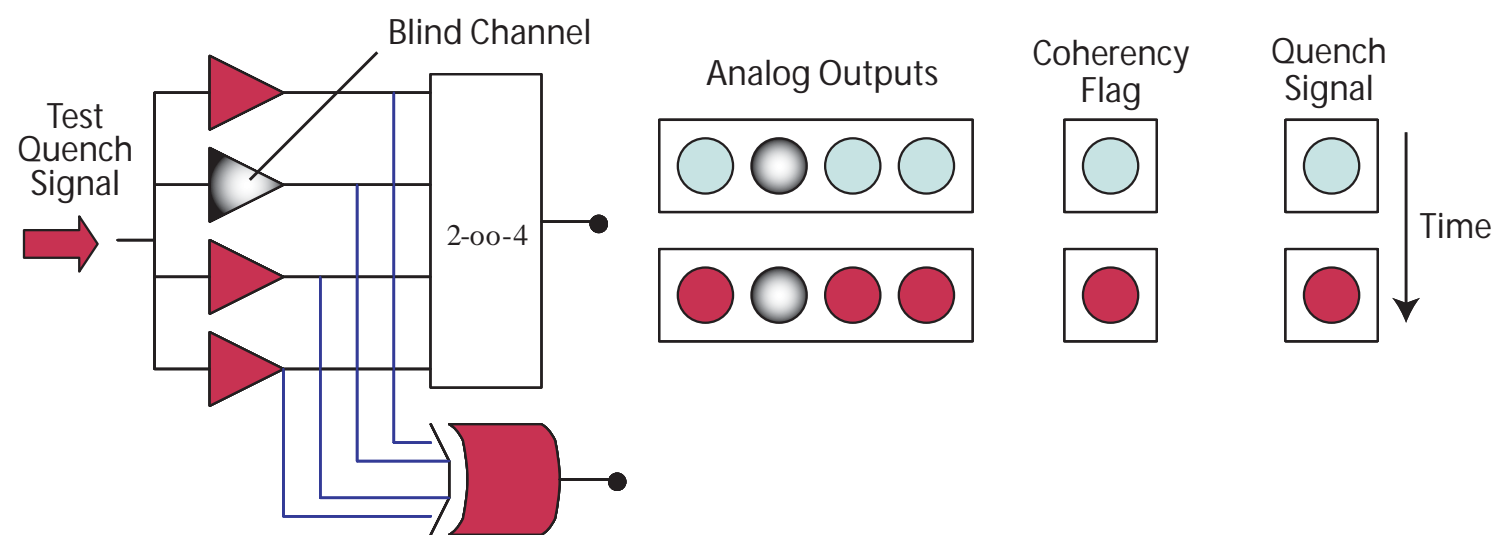

Figure 7.3: Quench test in a 2004 detector with one channel blind.

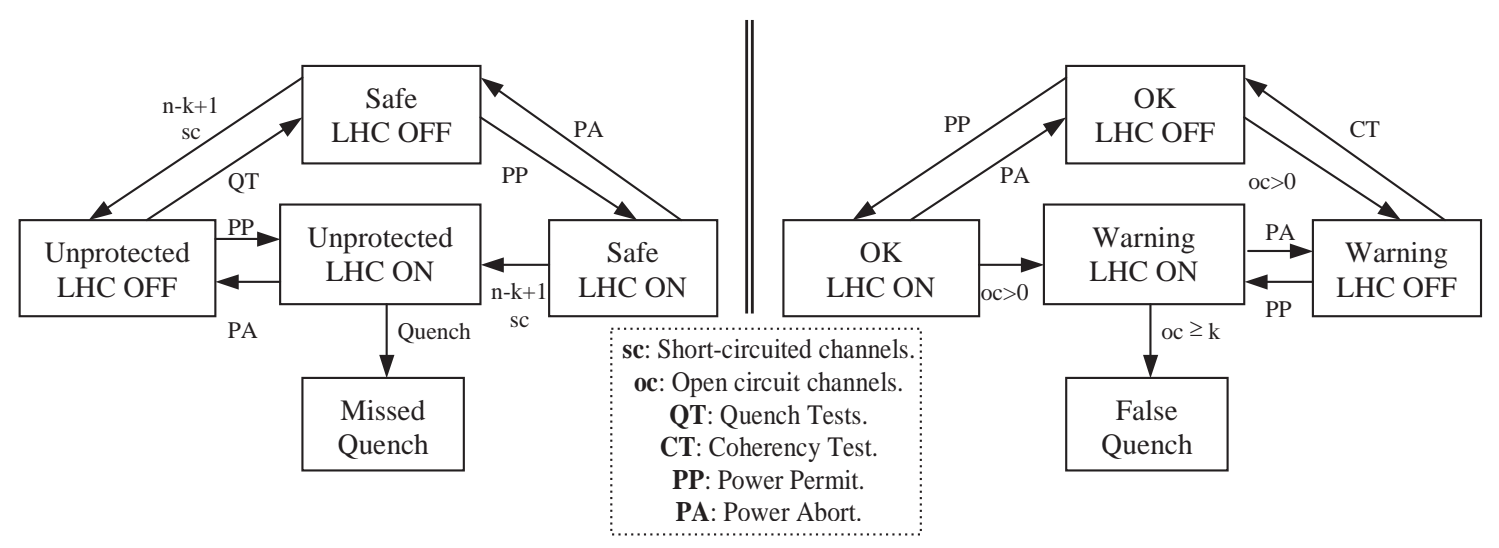

Figure 7.4: Markov diagrams for UM and FQ models of the DQQDL analog part. 


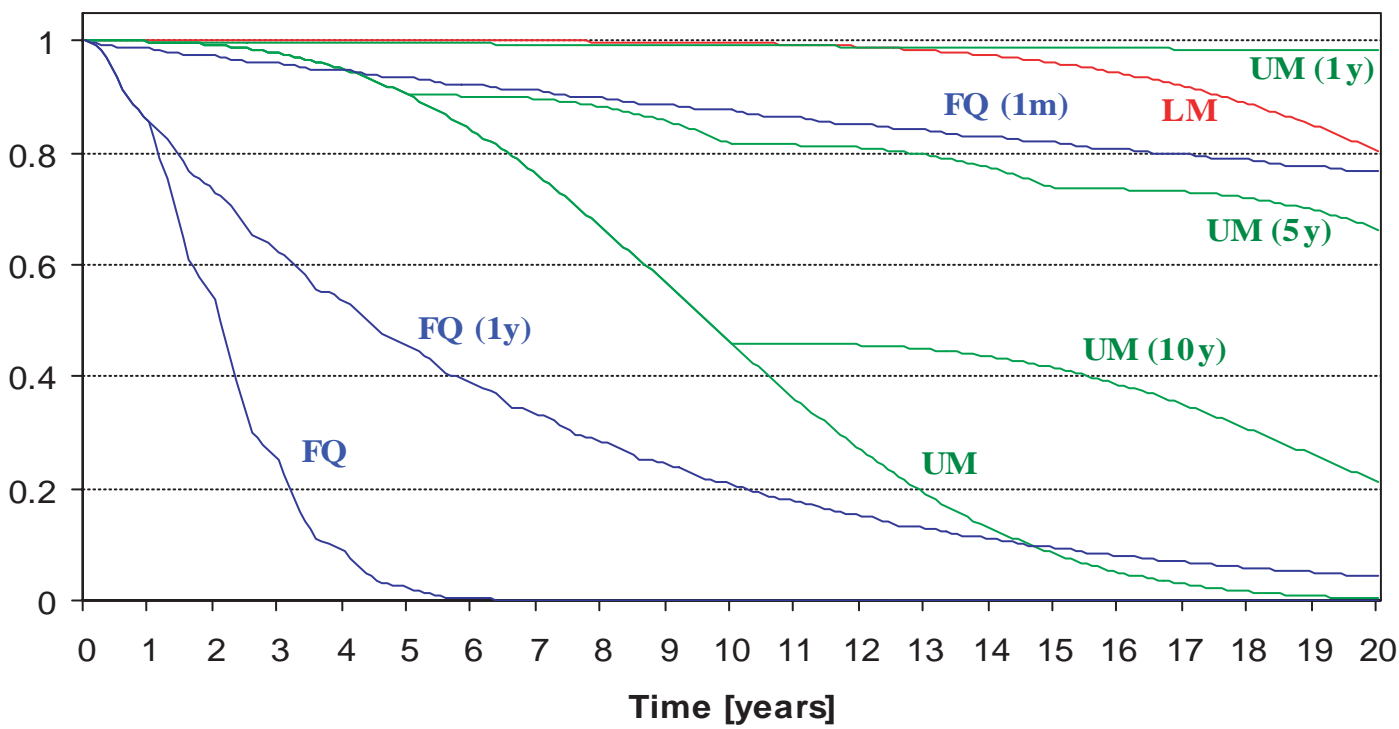

Figure 7.5: Example of the impact of CT and QT tests on the reliability of a detector with 2003 redundant scheme (UM: Unprotected Magnet, MQ: Missed Quench, FQ: False Quench). The number in brackets makes reference to the test frequency.

An example of the maintenance influence on the detector reliability (referred to false quenches, unprotected magnets and missed quenches) is represented in Fig. 7.5.

\subsubsection{Reliability Data of the Components}

All the local quench detector components will work under radiation. The ones that qualified after the radiation tests (see Appendix A) and will be used in the DQQDL final designs are listed in Table 7.6 together with their expected reliability.

\begin{tabular}{lccc}
\multicolumn{1}{c}{ Device } & Name & MTBF & Failure Rate \\
\hline Instrumentation Amplifier $^{1}$ & INA141AM & $1447329 h$ & $6.90927 \cdot 10^{-7} h^{-1}$ \\
Voltage Reference $^{1}$ & REF102AP & $4341989 h$ & $2.30307 \cdot 10^{-7} h^{-1}$ \\
Differential Amplifier $^{1}$ & INA105AM & $5644587 h$ & $1.7716 \cdot 10^{-7} h^{-1}$ \\
Comparator $^{1}$ & LM311N & $4341989 h$ & $2.30307 \cdot 10^{-7} h^{-1}$ \\
Power Supply $^{1}$ & Melchor 50WAC-DC & $880000 h$ & $1.136363 \cdot 10^{-6} h^{-1}$ \\
Wire $^{2}$ & & $26371308 h$ & $3.792 \cdot 10^{-8} h^{-1}$ \\
Logic $^{3}$ & CMOS gates & $0.021+0.0014 \cdot(\# \text { pins })^{1.08} h$ \\
\hline
\end{tabular}

Table 7.1: Failure rate data for DQQDL components.

\footnotetext{
${ }^{1}$ Data from manufacturer. Arrhenius formula used with $1 \mathrm{eV}$ activation energy.

${ }^{2}$ Data from MIL-HDBK-217F. $G_{B}$ [44]. Four soldering per wire (one at cold and three at warm), clip terminator.

${ }^{3}$ Data from MIL-HDBK-217F. $G_{B}$ : fix grounded [44].
} 


\subsubsection{Studied Topologies}

Next tables show the RESQP results for different feasible detection topologies and maintenance strategies. The unprotected magnet (UM), false quench (FQ) and missed quench (MQ) reliability have been computed for the 20-year LHC lifetime.

An example of reliability and availability results obtained with RESQP for two different topologies is shown in Fig. 7.6.
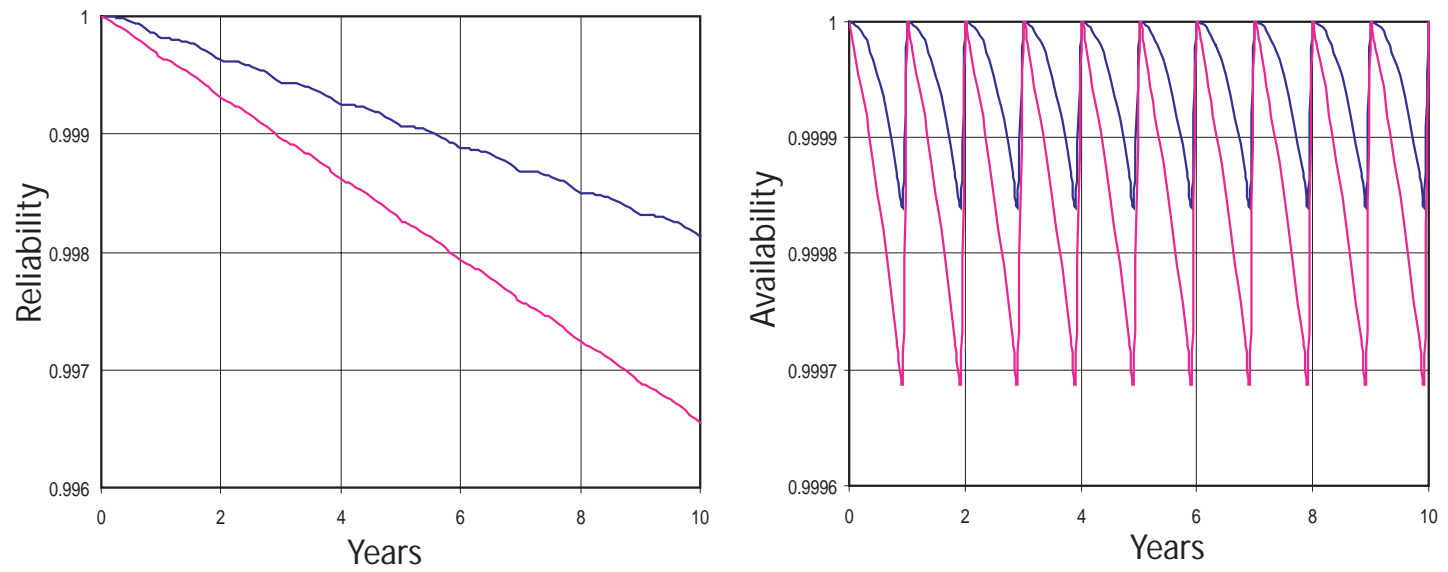

Figure 7.6: Reliability and availability with respect to MQ for two different quench detector topologies with yearly quench check.

The results show the expected number of failures for the 2016 local quench detectors in the machine applying Bernouilly distribution for a 95\% confidence level. Bernouilly is applied to 2016 trials with a probability $P_{S k}(t)$ of success. Where $P_{S k}(t)$ is the reliability with respect to the failure $S_{k}$. This actually assumes the hypothesis that a magnet can suffer a maximum of one false quench and one missed quench during all its lifetime. This hypothesis is very realistic for the best topologies with less than ten failures per year.

A maximum yearly operation time of $5110 \mathrm{~h}$ has been assumed, starting with a $70 \%$ during the first year and with a $10 \%$ yearly increment up to the maximum.

The number of missed quenches assumes 24 quenched magnets per operational week. This value does not mean that the machine performs such a number of power aborts, since the propagation from the quenched magnet to their neighbours is included. Nevertheless it is a pessimistic value, hence the results with only 6 quenched magnets per operational week have been included in brackets. The results below consider one independent voltage reference per analog channel.

\section{Redundant Analog Part}

The signals are treated by a k-oo- $n$ active redundancy. The instrumentation wires are shortcircuited (see Fig. 7.7). This reduces the false quench probability due to broken wires but unables checks of the wires. Since the voting system is not redundant, CT tests will not prevent about failures on the logic level.

The results for yearly quench checks and monthly coherent checks are listed in Table 7.2. 


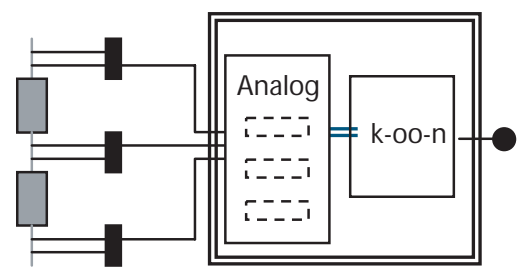

Figure 7.7: Quench detector with redundant analog part (k-oo-n).

\begin{tabular}{l|ccc|c} 
1y-1m & UM & FQ & MQ & Average Time [days] \\
\hline 1 oo1 & $233-277$ & $303-352$ & $38-58(7-17)$ & $1412(404)$ \\
1002 & $3-10$ & $408-462$ & $0-3(0-1)$ & $132(104)$ \\
2002 & $454-510$ & $189-229$ & $83-111(17-31)$ & $3155(715)$ \\
1003 & $2-8$ & $498-555$ & $0-2(0-1)$ & $137(123)$ \\
2003 & $11-32$ & $193-233$ & $0-5(0-2)$ & $114(72)$ \\
1004 & $2-8$ & $576-637$ & $0-2(0-1)$ & $188(140)$ \\
2004 & $18-32$ & $199-240$ & $1-7(0-3)$ & $157(87)$ \\
3004 & $24-41$ & $197-238$ & $2-8(0-3)$ & $187(87)$ \\
\hline
\end{tabular}

Table 7.2: DQQDL results with monthly CT and yearly QT.

It can be observed that increasing the number of channels (e.g. 2003 and 2oo4) does not necessarily involve less unprotected magnets. This is due to the higher complexity of the logic part when more channels are added.

Pareto analyses show that false quenches are mainly due to powering failures. As long as the power supply is not redundant, we have to live with these big numbers independently of the detection scheme chosen. The power redundancy issue is studied below.

The missed quenches of the best topologies are due to problems in the logic. In order to compensate this effect the following topologies have been considered.

\section{Redundant Analog and Logic Parts}

The logic circuit is now doubled and connected by an OR gate (see Fig. 7.8). This allows prevention of unprotected magnets via the quench checks also at the logic level, which improves the unprotected magnet (and consequently the missed quench) reliability. On the other hand, the number of false quenches will be increased since a failure in one of the parallel voting circuits will generate a spurious quench signal. The other operational characteristics remain as above.

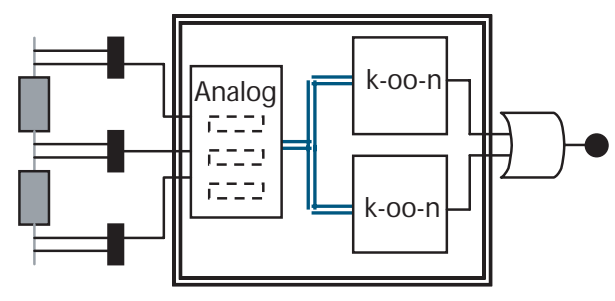

Figure 7.8: Quench detector with redundant analog and logic parts (k-oo- $n d)$. 


\begin{tabular}{c|ccc|c} 
1y-1m & UM & FQ & MQ & Average Time [days] \\
\hline 1oo1d & $233-277$ & $303-352$ & $38-58(7-17)$ & $1412(404)$ \\
1oo2d & $0-4$ & $409-463$ & $0-3(0-1)$ & $104(90)$ \\
2oo2d & $451-506$ & $191-232$ & $82-110(17-31)$ & $2732(716)$ \\
1oo3d & 0 & $499-557$ & 0 & 110 \\
2oo3d & $2-9$ & $198-239$ & $0-2(0-1)$ & $73(59)$ \\
1oo4d & 0 & $578-638$ & 0 & 126 \\
2oo4d & 0 & $210-252$ & 0 & 48 \\
3oo4d & $6-15$ & $207-249$ & $0-3(0-1)$ & $89(61)$ \\
\hline
\end{tabular}

Table 7.3: DQQDL results with monthly CT and yearly QT

The scheme $2004 d$ has a very good performance with a reliability against missed quenched above $99 \%$. The price to pay is a higher complexity in both the analog and the logic part. This very complex logic (around 20 logic gates needed per detector) may have bad consequences if the expected reliability data for the CMOS gates results to be too optimistic. The topology is negatively redundant in terms of false quenches due to the OR gate.

Along the following topologies the objective is to get reliability levels similar to those of $20 o 4 d$ with simpler schemes.

\section{Two Detectors in Series Redundancy}

The whole detector, with a simpler scheme (e.g. 1oo2) is duplicated and the cards are connected by an AND gate (wired logic). The checks will prevent UM and FQ failures at analog and digital level.

The broken wires can be detected since they are not short-circuited and each redundant wire is routed to a different card. In this way at least two wires must be lost to provoke a false quench signal. The coherency flag will warn from a broken instrumentation wire.

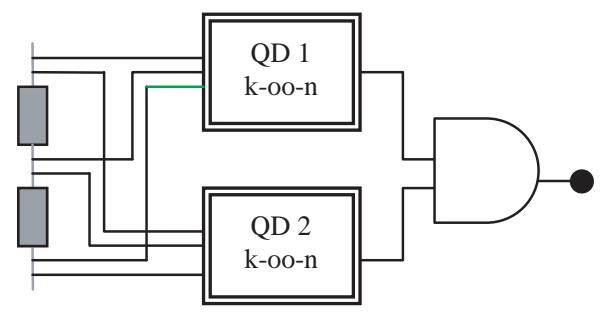

Figure 7.9: Quench detectors in series redundancy ( $k$-oo-n and $k-o o-n)$.

Although 1002 andloo2 has a slightly better performance than $20 o 4 d$ with respect to false quenches (mainly due to the much simpler logic), it has a limited reliability against missed quenches (56\% in the best case of 6 quenches per operational week). This weak MQ reliability makes the topology inadequate. 


\begin{tabular}{l|ccc|c} 
1y-1m & UM & FQ & MQ & Average Time [days] \\
\hline 1oo2and1oo2 & $8-19$ & $187-227$ & $0-4(0-2)$ & $99(71)$ \\
1oo3and1oo3 & $6-15$ & $187-227$ & $0-4(0-1)$ & $99(57)$ \\
2oo3and2oo3 & $26-24$ & $187-227$ & $2-7(0-3)$ & $197(85)$ \\
\hline
\end{tabular}

Table 7.4: DQQDL results with monthly CT and yearly QT

\section{Two Detectors in Parallel Redundancy}

In order to improve the unprotected magnet reliability, two detectors with redundancy only in the analog part are connected in parallel (see Fig. 7.10). The instrumentation wires are not shortcircuited and each one is routed to a different detector.

Quench checks prevent UM failures at analog and logical level, whereas coherency checks only ward off false quenches at analog stage, due to the OR link of the cards.

Broken wires are not detectable since only one wire disconnected is enough to generate the false quench signal.

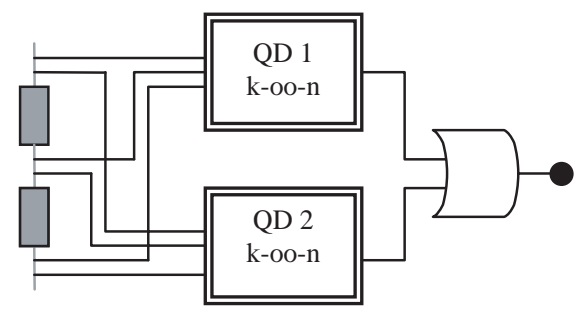

Figure 7.10: Quench detectors in parallel redundancy (k-oo-n or k-oo-n).

\begin{tabular}{l|ccc|c} 
1y-1m & UM & FQ & MQ & Average Time [days] \\
\hline 10o2or1oo2 & 0 & $581-642$ & 0 & 127 \\
2oo2or2oo2 & $3-11$ & $197-238$ & $0-2(0-1)$ & $73(59)$ \\
2oo3or2oo3 & 0 & $204-246$ & 0 & 46 \\
\hline
\end{tabular}

Table 7.5: DQQDL results with monthly $\mathrm{CT}$ and yearly QT

Although 2003 or $20 o 3$ shows the best performance, it is not remarkably better than $20 o 4 d$ and the complexity and space requirements increase due to the six channels needed.

The choice bust be taken between 2oo4d and 2oo2or2oo2. The first scheme shows the best performance with the used parameters. On the other hand, the second one is much simpler and its basic logic makes it more robust in front of possible variations on the failure rate of its logic part (which are the most uncertain components of the detector), as it will be seen in the sensitivity analysis.

The Pareto analysis reveals that missed quenches in the 2oo2or2oo2 topology are due to problems in the analog part. The reliability of these components can be tested by the quench checks. This means that higher frequency tests will imply better performance. 


\subsubsection{Sensitivity Analysis}

The local quench detector topology must be chosen between $2004 d$ and 2002 or $20 o 2$. The influence of higher logic failure rate than expected $\left(\lambda_{\log }=10 \lambda_{n o m}\right)$ and the QT frequency on the total missed and false quench reliability is shown in Table 7.6.

\begin{tabular}{|l|c|c||c|c|}
\cline { 2 - 5 } \multicolumn{1}{c|}{} & \multicolumn{2}{c||}{$\lambda_{\text {log }}=\lambda_{\text {nom }}$} & \multicolumn{2}{c|}{$\lambda_{\text {log }}=10 \lambda_{\text {nom }}$} \\
\hline MQ & $99.3 \%$ & $41 \%$ & $66 \%$ & $36 \%$ \\
QT=1y & 0 & $0-2$ & $0-1$ & $0-2$ \\
\hline MQ & $99.9 \%$ & $99.1 \%$ & $99.7 \%$ & $99 \%$ \\
QT=1m & 0 & 0 & 0 & 0 \\
\hline FQ (mean) & 240 & 217 & 433 & 258 \\
\hline & 20o4d & 20o2or2oo2 & 20o2d & 2oo2or2oo2 \\
\cline { 2 - 5 } & & &
\end{tabular}

Table 7.6: Comparison between the two topologies. Missed quench reliability for all the dipole quench detectors, expected missed quenches with a 95\% confidence level for yearly and monthly QT and mean of expected false quenches for monthly CT.

The logic failure rate has a big influence on the MQ and FQ reliability for the 2oo4d topology, whereas the behaviour of the protection scheme 2002 or 2002 is more stable to failure rate drifts. This is due to its simpler and checkable logic part.

If the quench test (QT) frequency is increased from yearly to monthly checks, the simpler $20 o 2$ or $20 o 2$ topology performs as well as $20 o 4 d$. This, together with the lower number of false quenches makes 2002 or 2002 the chosen solution.

In order to check the robustness of the model and to look for strong interaction between inputs, the sensitivity of the results to the input parameters has been studied. The study has been focused on the influence of the failure rate data of the components, the expected quench rate and the check frequency. The different failure rates have been simultaneously drifted up to 10 times their nominal values. A range of from yearly to monthly checks has been swept for the quench test frequency.

The probability of having less than one, two, three, and six quenches after 20 years of LHC operation, in function of the total failure rate (logic and analog part together) and the expected quench rate is plotted in Fig. 7.11 and Fig. 7.12 for yearly and monthly quench checks respectively. These figures show the big influence of the quench tests on the missed quench reliability. The 2oo2or 2002 detection scheme will hardly miss any quench if quench checks are carried out monthly.

The expected number of missed quenches after 20 years for the chosen topology with yearly and monthly QT are plotted in Fig. 7.13.

\subsubsection{Powering and False Quenches}

The large number of false quenches showed by all the topologies (above 10 false quenches per year) are mainly due to the power supply.

Since the powering is not redundant, the different checks on the system do not affect its reliability. Several solutions have been considered.

A first option is to power the two boards of the detector independently. This would have a negative effect on the 2002 or 2002 detector due to the OR gate. Hence the second best topology regarding MQ reliability must be used. If the boards of the 1 oo 2 and $1 \circ o 2$ detector are powered 
$M Q=0$

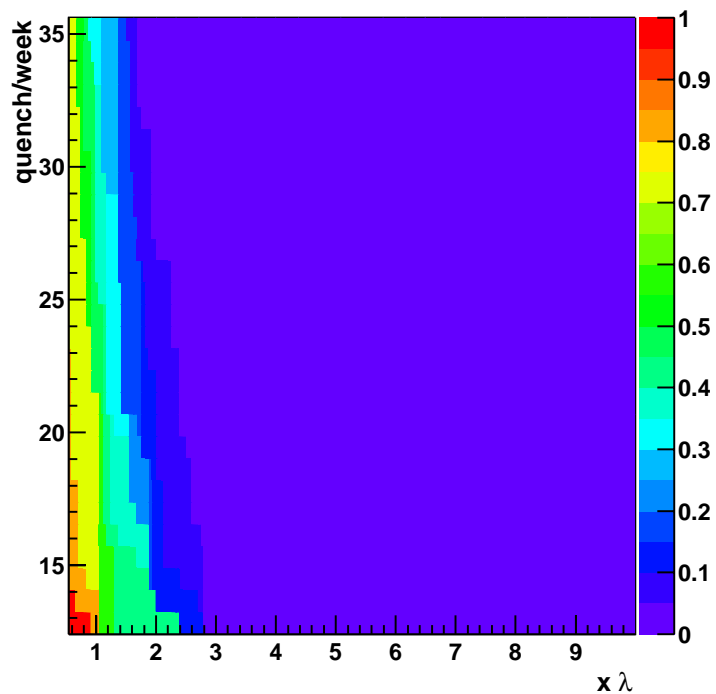

$M Q<=2$

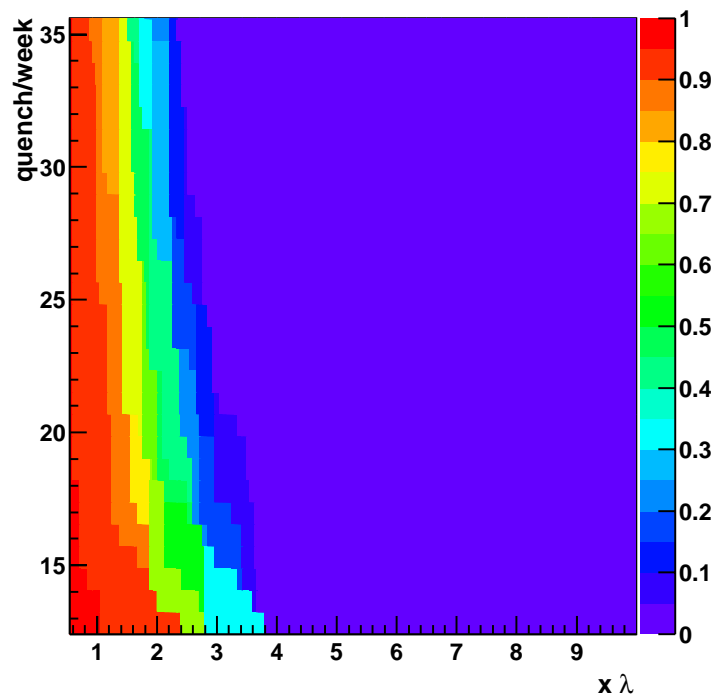

$M Q<=1$

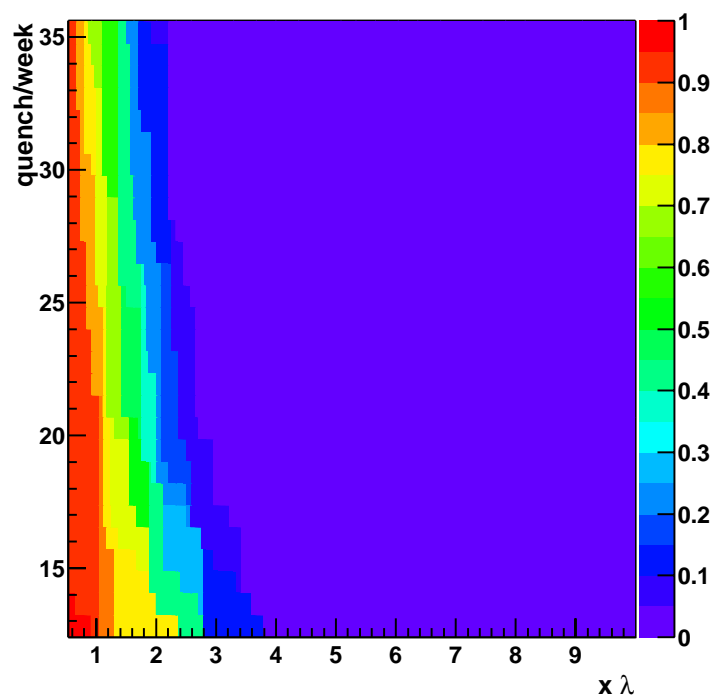

$M Q<=5$

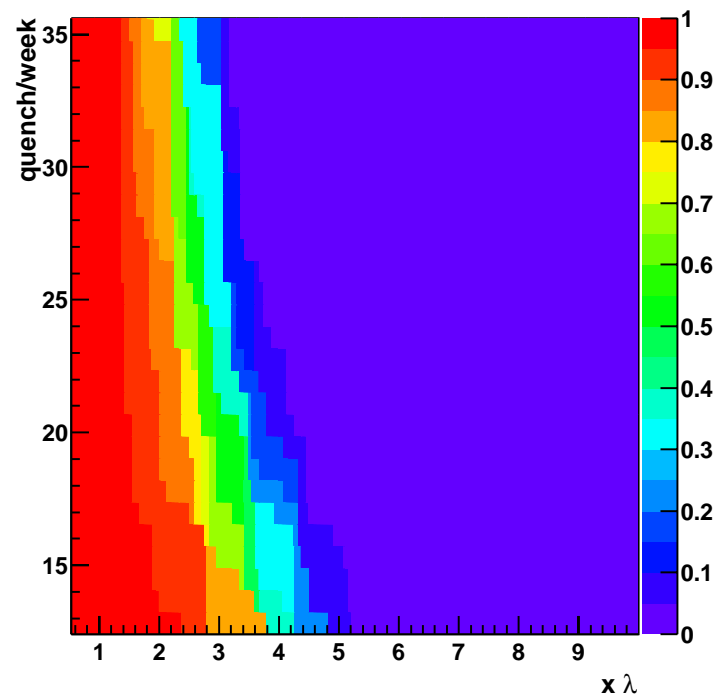

Figure 7.11: Probability of suffering less than one, two, three, and six missed quenches after 20 years in function of the total failure rate drift from the nominal value, $\lambda$, and the expected quench rate. 2002 or $20 o 2$ detection scheme with yearly QT.

by independent power supplies the number of false quenches will decrease due to the redundancy and, which is more important, it will almost vanish since the coherency checks will prevent from failures due to the powering. On the other hand, the cost of changing the detection topology will increase the missed quench probability.

A cash-flow analysis has been carried out [74] in order to check the benefit of doubling the number of power supplies for the DQQDL detectors and changing the detection topology from $2 o o 2$ or $20 o 2$ with single powering from 1 oo2and1oo 2 with redundant power supplies.

The performance of both options (mean values) are listed in Table 7.1.7. The total operation 

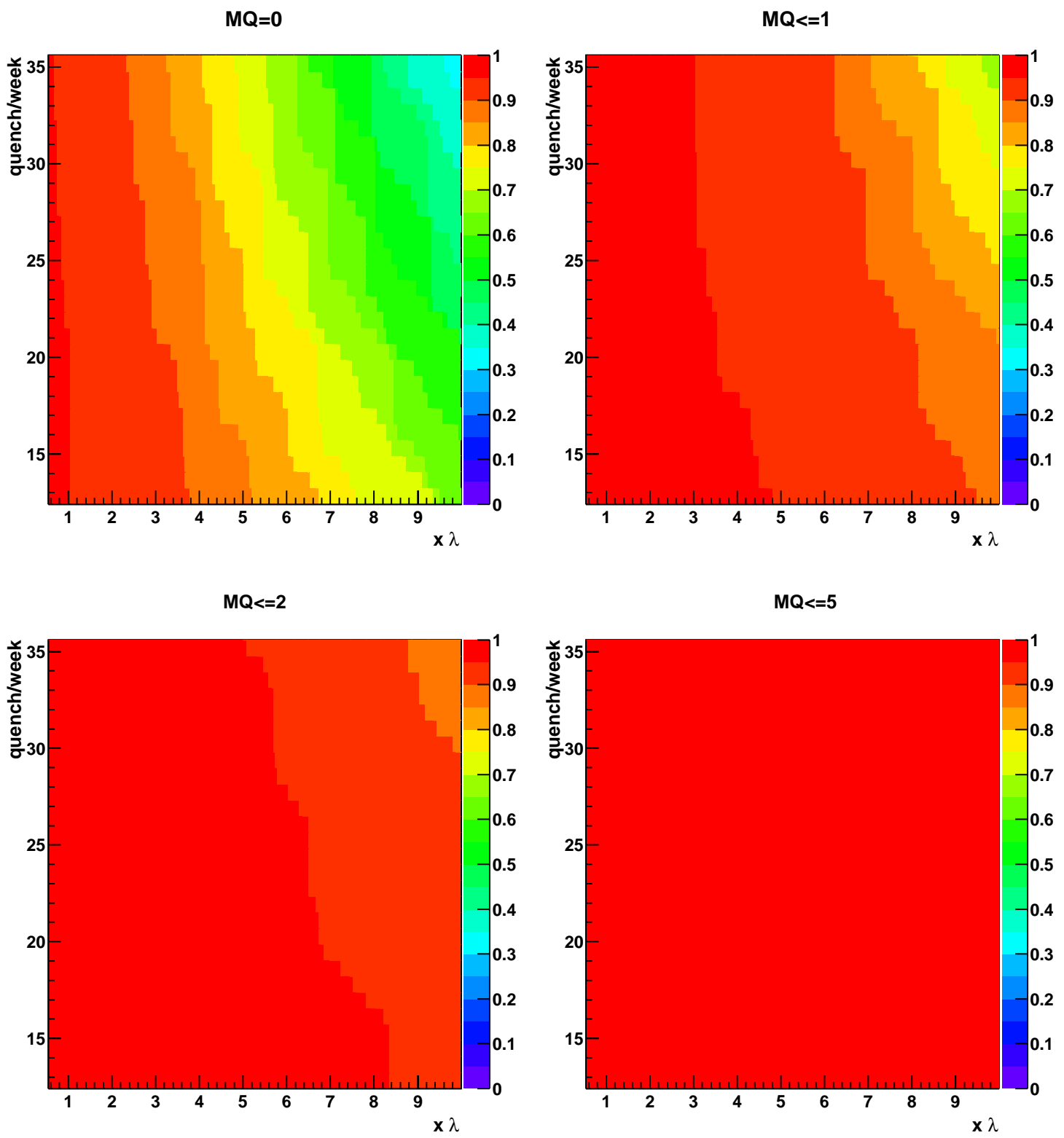

Figure 7.12: Probability of suffering less than one, two, three, and six missed quenches after 20 years in function of the total failure rate drift from the nominal value, $\lambda$, and the expected quench rate. 2002 or $20 o 2$ detection scheme with monthly QT.

time during the first years is of crucial importance, since the main decision parameter is the payback period of the investment. Hence, the study considers three different cases of actual operational time: $50 \%, 70 \%$ or $100 \%$ of the scheduled opeational time during the first year, with a $10 \%$ yearly increase up to the maximum.

The net present value (NPV) of an investment is defined as the present value of the investment's future net cash flows minus the initial investment (Eq. 7.1): 

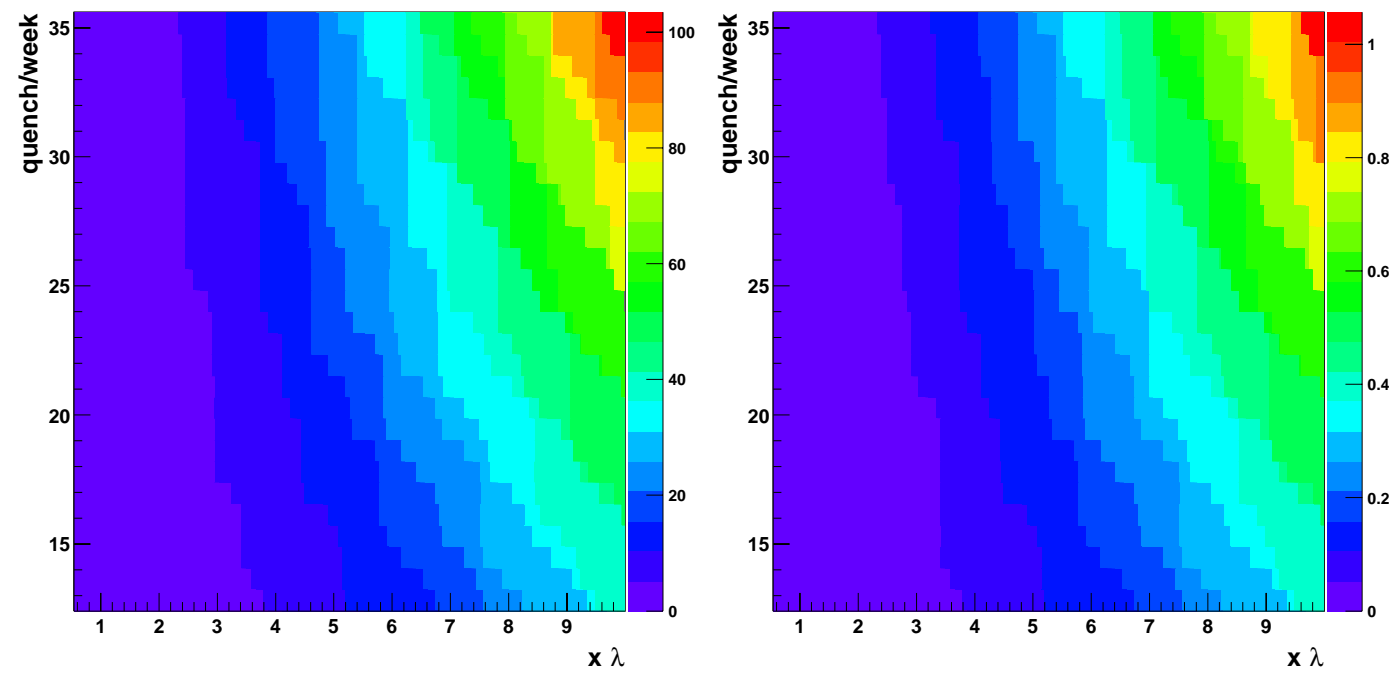

Figure 7.13: Sensitivity of missed quenches after 20 years for $20 o 2 o r 2 o o 2$ topology with yearly (left) and monthly (right) quench checks

\begin{tabular}{c|cccccc}
\multirow{2}{*}{ Scheme } & \multicolumn{2}{|c}{ Initial Operation 50\% } & \multicolumn{2}{c}{ Initial Operation 70\% } & \multicolumn{2}{c}{ Initial Operation 100\% } \\
& FQ & MQ & FQ & MQ & FQ & MQ \\
\hline 2oo2or2oo2 - 1 PS & 209 & 1 & 219 & 1 & 225.5 & 1 \\
10o2and1002 - 2 PS & 12.5 & 2 & 13 & 2 & 14 & 2 \\
\hline
\end{tabular}

Table 7.7: Mean values for the two studied topologies to reduce false quenches due to powering.

$$
N P V=\sum_{i=\Delta}^{\Delta+T} \frac{C F}{(1+r)^{i}}-I_{0}
$$

where $r$ is the discount or capitalisation rate, $\Delta$ the time in years from the investment payment to the LHC operation start, $T$ the number of operation years and $I_{0}$ the investment (i.e. 2016 power supply: I=1.2096 MCHF [75]). CF represents the cash-flow, which is the net profit plus amortisation of the investment. In our case it will be due to the saved costs due to the lower downtime (Eq. 7.2).

$$
\begin{aligned}
C F= & \left(F Q^{1 P S}-F Q^{2 P S}\right) C_{s}(5 h)+\left(M Q^{1 P S}-M Q^{2 P S}\right)\left(C_{s}(672 h)+C_{m}\right)+ \\
& \left(P S_{s}^{1 P S}-P S_{s}^{2 P S}\right) C_{P S}
\end{aligned}
$$

with $F Q^{1 P S}, M Q^{1 P S}$ and $F Q^{2 P S}, M Q^{2 P S}$ being the false and missed quenches with 2oo2or2oo2 and 1oo2andloo2 respectively. $C_{s}$ is the downtime hour cost, $C_{m}$ the price of a magnet, and $C_{P S}$ the price of one quench detector power supply. $P S_{S}$ are the required spare power supplies for each topology. 
The discount rate value recommended at CERN for investment studies is between $5 \%$ and $6 \%$ [76]. Since the current financial situation of the Institute recommends to delay as long as possible the investments, the highest value will be used: $r=0.06$.

The CERN collaboration with India may imply sharing of the quench detector costs, this would mean that the investment $I_{0}$ would be halved. The study has been carried out for both situations: CERN paying the full cost and only the $50 \%$.

Next plots (Fig. 7.14 and Fig. 7.15) show the expected pay-back periods of the investment in function of the the magnet cost $C_{m}$ and the cost of unscheduled downtimes per hour $C_{s}$. A delay from payment to operation start up of 2 years has been considered ( $\Delta=2$ years).
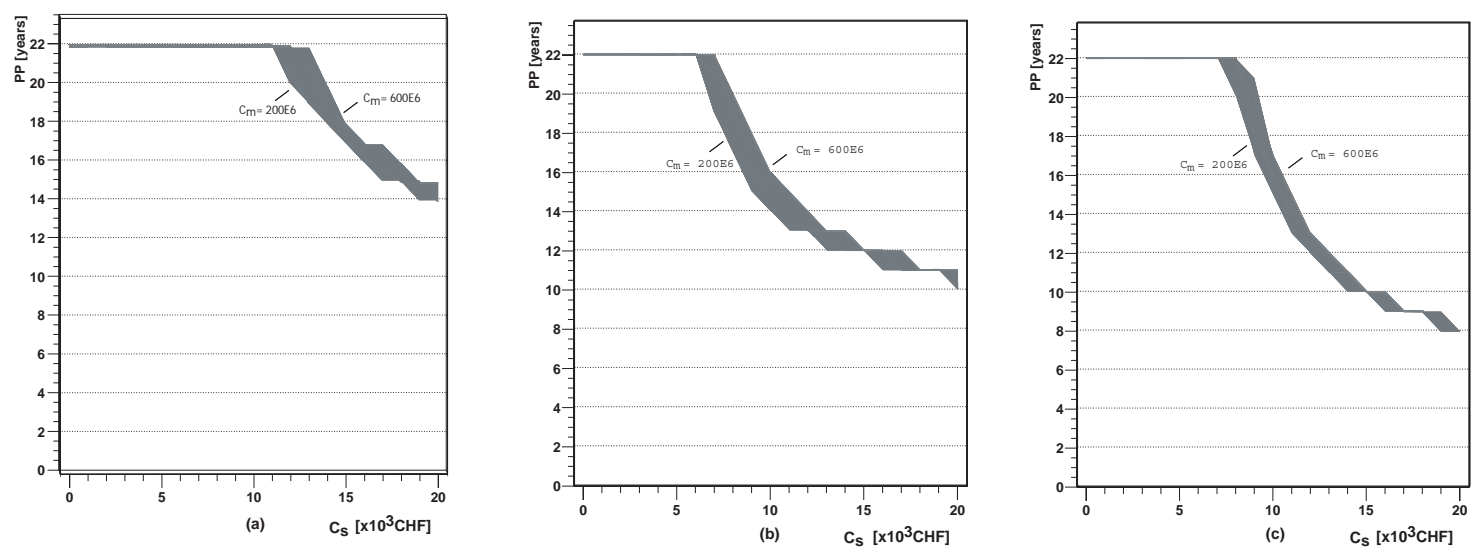

Figure 7.14: Pay-back period (in years) for $100 \%$ of the investment covered by CERN ( $\mathrm{r}=0.06, \Delta=2$ years) with first year operation of 50\% (a), 70\% (b), and 100\%(c) of the scheduled time.
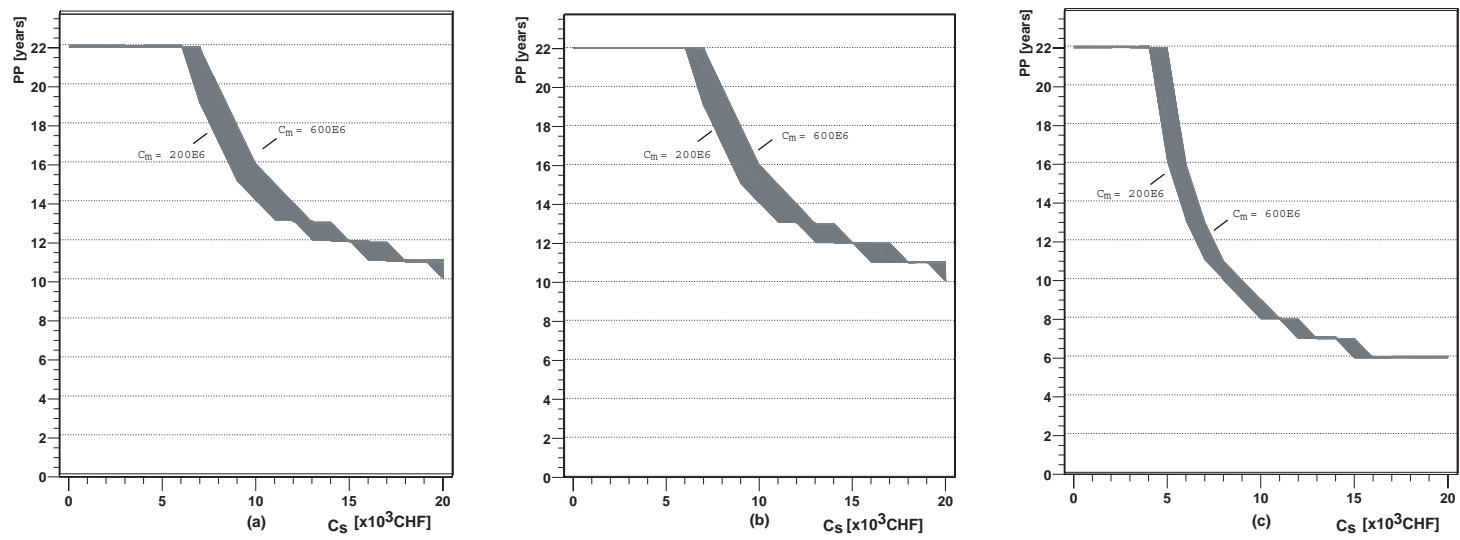

Figure 7.15: Pay-back period (in years) for $50 \%$ of the investment covered by CERN ( $\mathrm{r}=0.06, \Delta=2$ years) with first year operation of 50\% (a), 70\% (b), and 100\%(c) of the scheduled time.

While the magnet cost do not strongly affect on the study result, it remarkably depends on the downtime cost. Table 7.8 summarises the pay-back period for a downtime cost of 10,000 and 15,000 Swiss Francs per hour. The value of $C_{m}$ has been considered between 100,000 and 600,000 Swiss Francs. 


\begin{tabular}{c|cccc}
\multirow{2}{*}{ Operation } & \multicolumn{4}{|c}{ Pay-back Period from Investment [years] } \\
& 100\% Investment & 50\% Investment \\
& $C_{s}=10$ & $C_{s}=15$ & $C_{s}=10$ & $C_{s}=15$ \\
\hline from 50\% & - & $17-18$ & $14-15$ & 12 \\
from 70\% & $18-21$ & $12-13$ & $10-11$ & $8-9$ \\
from $100 \%$ & $16-17$ & 11 & $8-9$ & $6-7$ \\
\hline
\end{tabular}

Table 7.8: Expected pay-back period of the investment needed to double the DQQDL power supplies, in function of $C_{s}\left(10^{3} \mathrm{CHF}\right.$.)

The investment will not be rewarding until six or seven years in the very best conditions. Assuming less optimistic conditions, pay-back period becomes longer than ten years. Moreover, the risk of missed quenches will increase, which may have a political cost not reflected on the results. Therefore, the solution of changing the 2oo2or2oo2 topology by 1002 andloo 2 with two power supplies has been rejected: the decrease of false quenches is not enough to cover the required investment due to the increase of missed quench probability.

Another solution is to power the chosen 2oo2or2oo2 with two power supplies connected in parallel. The detector will share the two power supplies and if one fails the other will cope with the needs of both boards. This will not decrease the needed investment but will keep constant the number of missed quenches.

The coherency flag must be modified in order to include the status of the two power supplies to its inputs. In this way the coherency check is able to prevent false quenches provoked by the powering.

With this solution the number of false quenches drops from 10 to less than 1 per year. Due to the large investment required to double all the power supplies, it has been decided to install one power supply per local quench detector and keep the necessary space to install a redundant unit if operation experience demonstrates its needfulness.

RESQP code will be the decision tool to evaluate the necessity of introducing the redundant powering after few years of LHC operation (see Chapter 10).

\subsection{Inner Triplet (DQQDT) and Insertion Magnet (DQQDI) Quench Detectors}

A common quench detector design has been used for the main magnets at the long straight sections. These magnets can be divided in three families:

- Matching Magnets: they form the dispersion suppressor line. They are $6 \mathrm{kA}$ twin aperture quadrupoles. In the machine they can be individually powered (e.g. MQM, MQML and $\mathrm{MQY}$ ) or connected in couples (MQM+MQMQ, MQY+MQY and MQM+MQC).

- Separation Magnets: these are the elements in charge to introduce the two parallel beams into a common beam pipe before collisions. They are $6 \mathrm{kA}$ dipoles of twin (MBRA, MBRB, MBRC) or single (MBRS, MBRX) aperture. MBRC and MBX are powered individually and the other three in couples connected in series. 
- Inner Triplet: Four single aperture quadrupoles at each side of the experiments (MQXA and MQXB) form the inner triplet. These magnets are in charge of focusing the beam before the insertion into the experiment as much as possible. They play the most important role regarding the LHC luminosity. Their complex powering is represented in Fig. 7.16.

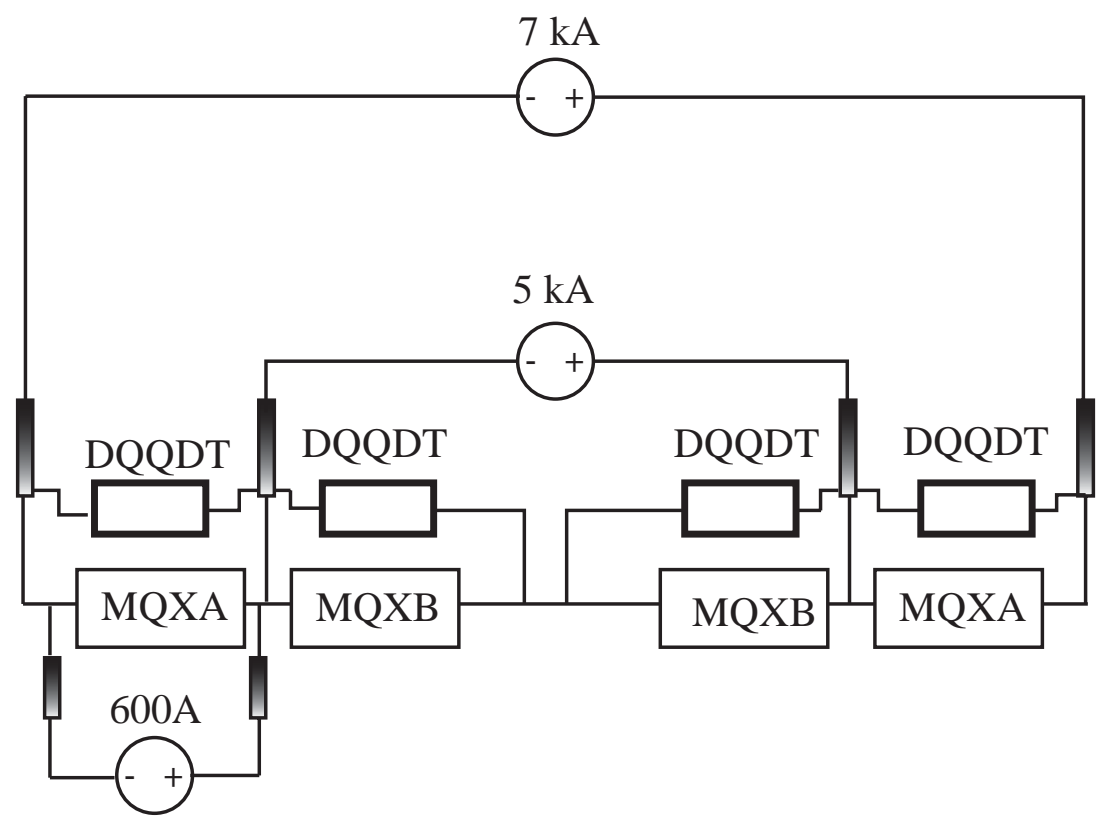

Figure 7.16: Inner triplet circuit with location of the DQQDT quench detectors.

Since the detector design must be the same for the three families above, a common expected quench rate has been averaged. As it was done for the main magnets, the maximum expected quench rate has been computed from the maximum number of expected quenches used for the hardware design $[24,25,26]$. The quenches seen by the detectors of each circuit are listed in Table 7.9. The average value is also shown. In case of a quench, all the quench heater power supplies of the circuit will be fired.

\subsubsection{Detector Design}

The DQQDT and DQQDI quench detectors are based on a floating bridge, but, in contrary to the main dipole and quadrupole magnets, the superconducting busbar is also part of the bridge. The signals from each aperture will be therefore not balanced.

A digital signal processor (DSP) allows the implementation of digital filters and the compensation of inductances. The schematic circuit of a single channel detector is represented in Fig.7.17.

Even without quench generation, the input differential voltages are not equal. The signals are independently amplified (with gains of 1,10, 100 or 1,000) and, after being galvanically isolated and digitallised, they are treated by the microprocessor, which has been provided with the needed inductance tables.

Although there are two wires routed out from the cryomass for each voltage, in contrary to the main magnets, they take the signal from the same voltage tap. Hence, there is redundancy on the instrumentation wires but not on the connection to the coil. 


\begin{tabular}{lccccc} 
Circuit & $\begin{array}{c}\text { Magnets } \\
\text { per Circuit }\end{array}$ & $\begin{array}{c}\text { Circuits } \\
\text { in LHC }\end{array}$ & $\begin{array}{c}\text { Detectors } \\
\text { in LHC }\end{array}$ & $\begin{array}{c}\text { Quenches per } \\
\text { Detector (20 years) }\end{array}$ & $\begin{array}{c}\text { Lifetime } \\
\text { [years] }\end{array}$ \\
\hline MBRX & 1 & 4 & 4 & 10 & 20 \\
MBRC & 1 & 8 & 16 & 5 & 20 \\
MBRS & 4 & 2 & 4 & 10 & 20 \\
MBRA + MBRB & 2 & 2 & 4 & 10 & 20 \\
MQM + MQMC & 2 & 4 & 8 & 10 & 20 \\
MQM & 1 & 4 & 8 & 5 & 20 \\
MQM + MQM & 2 & 28 & 56 & 10 & 20 \\
MQML & 1 & 64 & 128 & 5 & 20 \\
MQY & 1 & 16 & 32 & 5 & 20 \\
MQY + MQY & 2 & 12 & 24 & 10 & 20 \\
MQXA & 2 & 8 & 16 & 28.5 & 7 \\
MQXB & 2 & 8 & 16 & 30 & 6 \\
\hline Total & & $\mathbf{1 6 0}$ & $\mathbf{3 1 6}$ & $\mathbf{9 . 0 4}$ & \\
\hline
\end{tabular}

Table 7.9: Insertion and inner triplet circuits of the LHC.

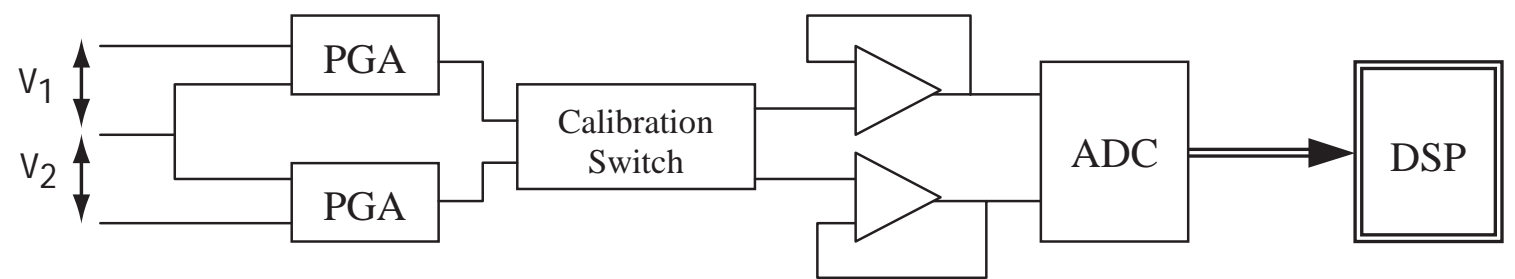

Figure 7.17: One-channel DQQDI detector (PGA: Programmable Gain Amplifier, ADC: Analog Digital Converter, DSP: Digital Signal Processor).

The scheme represented in Fig. 7.17 is not redundant, and a failure in any of its components will generate a false quench or an unprotected magnet state.

The failure mode and the maintainability options are the same than for the DQQDL detectors. However, the inputs of the coherency flag must be properly chosen. In this case the comparison must not be done between the output of the amplifiers, since they will have different outputs, but between the outputs of the microprocessors.

\subsubsection{Reliability Data of the Components}

Table 7.10 shows the nominal reliability data for the different elements of the 180 DQQDI and DQQDT detectors.

Two TMA (DC-DC) power supplies are needed per DSP but one TMS (AC-DC) is enough for powering two detectors. The models studied below will consider redundancy in AC-DC powering only if it is needed from a FQ reliability point of view. 


\begin{tabular}{lccc}
\multicolumn{1}{c}{ Device } & Name & MTBF & Failure Rate \\
\hline Programmable Gain Amp. $^{4}$ & PGA204AU & $1447329 h$ & $6.90927 \cdot 10^{-7} h^{-1}$ \\
Operational Amplifier $^{4}$ & OPA2277UA & $2542916 h$ & $3.932482 \cdot 10^{-7} h^{-1}$ \\
DSP $^{5}$ & TMS320C6211 & $799795 h$ & $1.25032 \cdot 10^{-6} h^{-1}$ \\
Power Supply (AC-DC) $^{6}$ & Traco TMS1505C & $250000 h$ & $4.0 \cdot 10^{-6} h^{-1}$ \\
Power Supply (DC-DC) $^{6}$ & Traco TMA & $>2000000 h$ & $5.0 \cdot 10^{-7} h^{-1}$ \\
Wire $^{7}$ & & $26371308 h$ & $3.792 \cdot 10^{-8} h^{-1}$ \\
Logic $^{5}$ & CMOS gates & $0.021+0.0014 \cdot(\# \text { pins })^{1.08} h$ \\
\hline
\end{tabular}

Table 7.10: Failure rate data for the components of the DQQDI and DQQDT detectors.

\subsubsection{Studied Topologies}

The different detection topologies analysed for this and the following detectors of this chapter are represented in Fig. 7.18.

The different schemes have been simulated with RESQP for several QT and CT check frequencies. It is important to notice that, due to the high amount of events (mainly false quenches generated by the power supplies) with respect to the number of detectors (i.e. 180), it can not be assumed anymore that one magnet will not suffer more than one false quench and one missed quench during all its lifetime. This has been corrected by running recurrently the code. The number of events occurred at the $(i-1)$-th iteration is taken as number of detectors at the $i$-th iteration. $Q_{m}^{i}$ is defined as the number of magnets that have suffered at least $i$ events above a total number of $m$ magnets, and $Q_{M}$ as the amount of events experienced by the $M$ magnets (see Eq. 7.3).

$$
\begin{aligned}
Q_{M}^{1} & \longrightarrow \text { Value given by RESQP } \\
Q_{Q_{M}^{1}}^{1} & =Q_{M}^{2} \\
Q_{Q_{M}^{2}}^{1} & =Q_{M}^{3} \\
\vdots & \\
Q_{Q_{M}^{3}}^{1} & =Q_{M}^{i} \\
Q_{M} & =\sum_{i=1}^{\infty} i\left(Q_{M}^{i}-Q_{M}^{i+1}\right)
\end{aligned}
$$

Table 7.11 lists the FQ, UM and MQ reliability of a single detector and the expected number of events after 20 years of operation with a $95 \%$ confidence level. The expected missed quenches for a more realistic quench rate ( $25 \%$ of the nominal) are shown in brackets. Maintainability consists on monthly coherency checks and yearly quench checks.

The topology 1oo2and1oo 2 with boards independently powered shows a very good performance against false quenches and a rather acceptable missed quench reliability. Nevertheless, the space restrictions in the area do not allow such a complex detector.

\footnotetext{
${ }^{4}$ Data from manufacturer. Arrhenius formula used with $1 \mathrm{eV}$ activation energy.

${ }^{5}$ Data from MIL-HDBK-217F. $G_{B}$ : fix grounded [44].

${ }^{6}$ Data from manufacturer.

${ }^{7}$ Data from MIL-HDBK-217F. $G_{B}$ [44]. Four soldering per wire (one at cold and three at warm), clip terminator.
} 


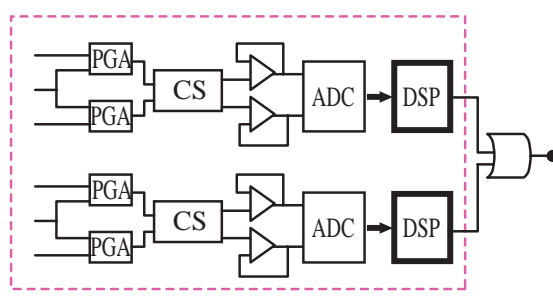

1002 - 1TMS

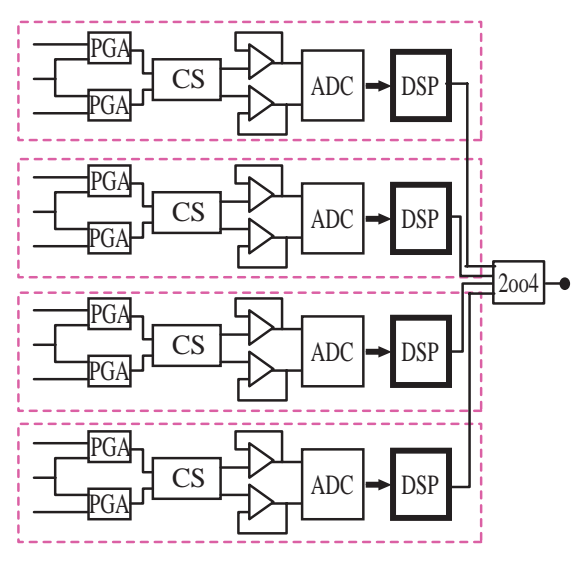

2004 - 4TMS

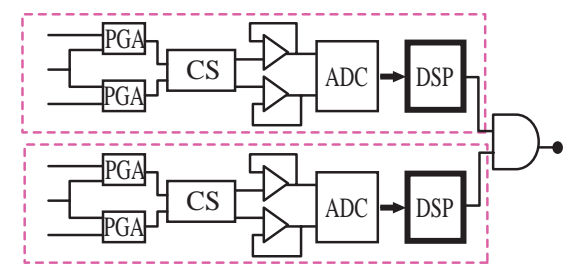

2002 - 2TMS

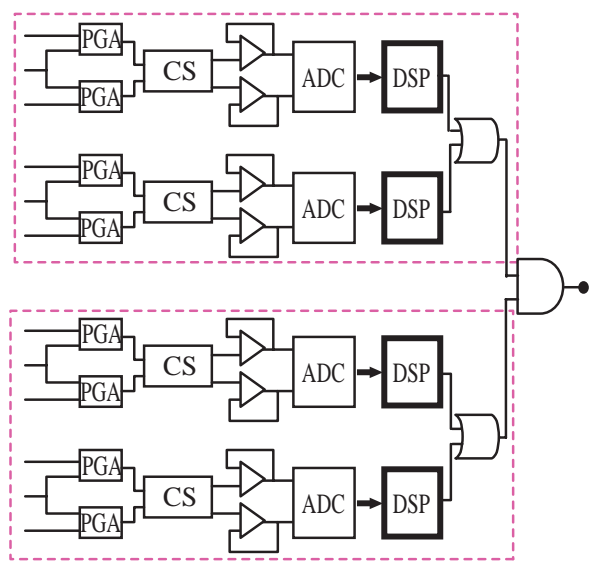

1002and1002 - 2TMS
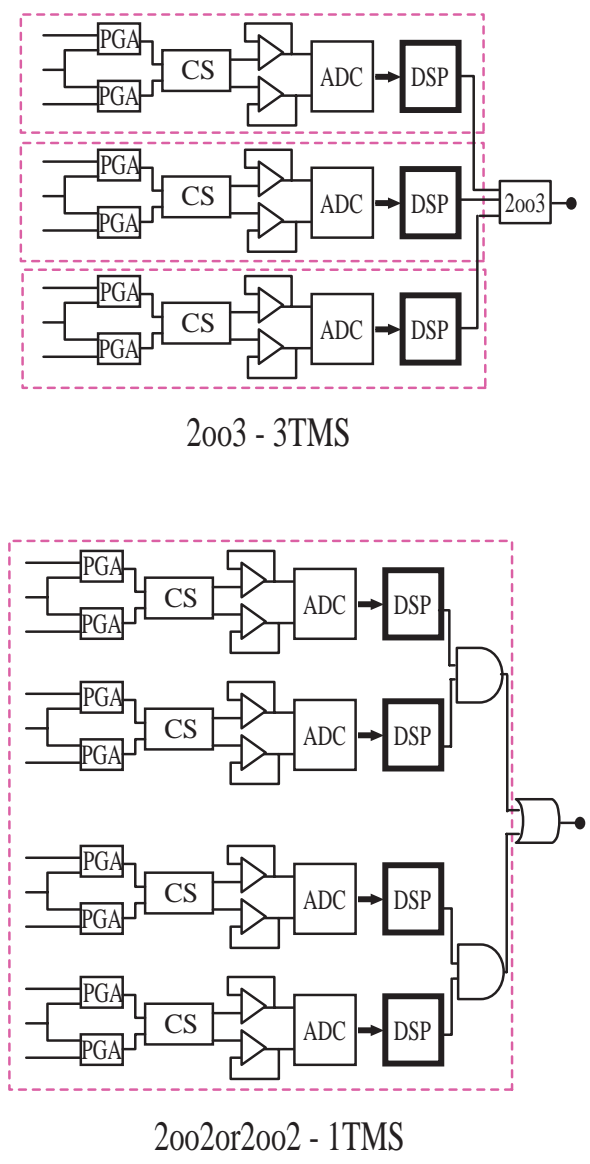

Figure 7.18: Different detection topologies analysed for the DQQDI and DQQDT quench detectors. The dashed lines represents the commonly powered components (TMS: power supply). 


\begin{tabular}{|c|c|c|c|c|c|c|c|}
\hline \multirow[b]{2}{*}{ Scheme } & \multicolumn{2}{|c|}{ UM } & \multicolumn{2}{|c|}{ FQ } & \multicolumn{2}{|r|}{ MQ } & \multirow[b]{2}{*}{ Av. Time $[\mathrm{d}]$} \\
\hline & Rel. & Events & Rel. & Events & Rel. & Events & \\
\hline 10o1-1TMS & 0.7412 & $88-143$ & 0.6363 & $145-231$ & 0.9614 & $8-9(1-5)$ & $417(123)$ \\
\hline 1oo2-1TMS & 0.9931 & $0-4$ & 0.5813 & $183-289$ & 0.9993 & $0-1(0)$ & $63(49)$ \\
\hline 2oo2-2TMS & 0.5480 & $210-231$ & 0.9950 & $0-3$ & 0.9229 & $18-36(3-10)$ & $756(182)$ \\
\hline 2oo3-3TMS & 0.9815 & $3-10$ & 0.9901 & $1-5$ & 0.9981 & $0-2(0-1)$ & $28(14)$ \\
\hline 2oo4-4TMS & 0.9870 & $2-7$ & 0.9901 & $4-12$ & 0.9981 & $0-1(0-1)$ & 15 \\
\hline 2oo2or2oo2-4TMS & 0.9826 & $3-10$ & 0.9886 & $1-6$ & 0.9984 & $0-1(0-1)$ & 29 \\
\hline 1ooand1oo2-2TMS & 0.9862 & $2-7$ & 0.9906 & $1-5$ & 0.9986 & $0-1(0-1)$ & 29 \\
\hline
\end{tabular}

Table 7.11: DQQDI and DQQDT reliability and expected failures (95\% confidence level) after 20 year with monthly CT and yearly QT.

Another possible solution would be 2003 with three power supplies, but its missed quench probability is not small enough even when the most optimistic quench rate is used. Pareto diagrams show that this is mainly due to failures in the complex logic part.

The chosen option is the 1oo2 topology. It shows a very low MQ probability, which almost vanishes at low quench rates, and its simple design is perfectly suitable to the available space in the tunnel.

The problem of such a simple topology is the big influence of the powering trips on the FQ reliability. An independent powering for each channel will only increase the number of false quenches.

A common powering by two shared power supplies will increase the reliability of a single detector against false quenches from $58 \%$ to $74 \%$, which implies a decrease of spurious quenches from 138-289 to 89-145 (monthly power supply checks). As can be seen in the Pareto plots of Figure 7.19 , the false quenches in redundantly powered detectors are mainly due to failures in the microprocessor and the analog part.
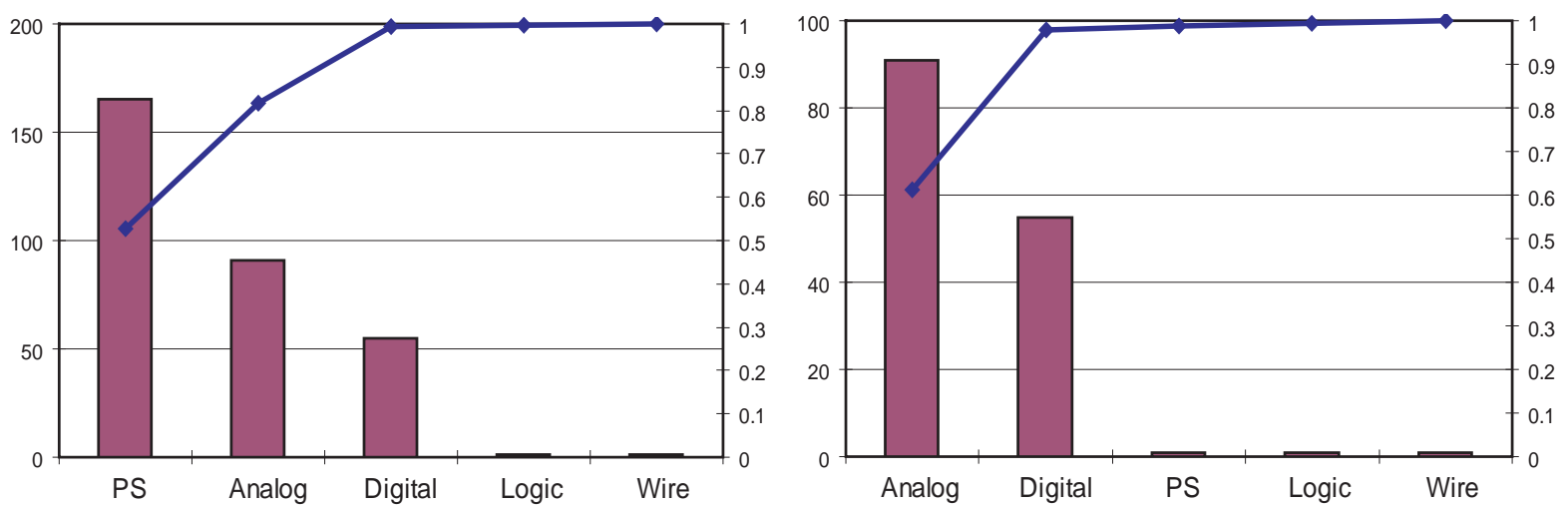

Figure 7.19: Pareto analysis of false quenches in DQQDI and DQQDT detectors for 1002 detection scheme without redundant powering (left) and with shared power supplies (right). Monthly CT and yearly QT have been considered.

It is important to remark that, with the chosen topology, the coherency tests will not worthless 
unless they are modified in order to monitor the status of the power supplies, which are the only redundant components in terms of false quench reliability.

\subsection{Corrector Magnet Quench Detectors (DQQDG)}

The 352 DQQDG detectors will protect the LHC corrector circuits. There exists a large variety of corrector magnets and circuits. While some circuits are formed by a single magnet (e.g. MQTL), others comprise up to 154 elements (e.g. MCS). These differences between circuits is a handicap for defining the quench rate and, above all, the failure costs.

\subsubsection{Quench Rate and Failure Costs}

The quench rate is a very difficult parameter to estimate because the corrector magnets have been designed with a high temperature margin, which almost vanishes the quench probability. Nevertheless, a failure rate is needed as starting point in order to optimise the detection topology. Two hypothesis have been assumed [77].

- Hypothesis 1: The quench probability is the same for each corrector magnet. Hence, the failure rate of the circuit (i.e. failure rate seen by the detector) depends on the number of magnets of the circuit.

- Hypothesis 2: The circuits with largest number of magnets (i.e. MCS) will suffer one quench per operational year. The failure rates for the other circuits are computed from this value.

The circuits with most magnets are the sextupole spool pieces (MCS), each one formed by 154 magnets. Therefore, the quench rate of every corrector magnet in the LHC will be:

$$
\mu_{\text {magnet }}^{M C S}=\mu_{\text {magnet }}^{L H C}=\frac{1}{154}=0.006493 \text { year }^{-1}
$$

From this value, the average quench rate per detector can be computed. The results are listed in Table 7.12. The uncertainty of these parameters is rather high, hence a broad sensitivity analysis of the reliability results to the quench rate will be required.

The computation of failure costs also needs to assume several hypothesis. The effect of a missed or a false quench on a magnet will be different depending on the the circuit protection and its function. The corrector circuits are protected in three different ways:

- Energy extraction and parallel resistor.

- Only energy extraction.

- Protection from the power converter.

A failure of the quench detector will have a major damage on the circuits protected only by the energy extractors. On the other hand, the circuits protected by the power converter can manage to survive after a non detected quench. The corrector circuits combining active (extraction) and passive (resistor) protection will be compromised if the quench is not detected, since the resistor is not able to carry out the protection by itself. 


\begin{tabular}{lcccc} 
& $\begin{array}{c}\text { Magnets } \\
\text { per Circuit }\end{array}$ & Circuits & $\begin{array}{c}\text { Quenches per } \\
\text { Detector }(20 \mathrm{y})\end{array}$ & Lifetime \\
\hline MCS & 154 & 16 & 20 & 20 \\
MCD & 77 & 16 & 10 & 20 \\
MCO & 77 & 16 & 10 & 20 \\
MO & 8 & 16 & 1.0389 & 20 \\
MO & 13 & 16 & 1.6883 & 20 \\
MSS & 4 & 16 & 0.5194 & 20 \\
MS & 10 & 32 & 1.2987 & 20 \\
MS & 12 & 32 & 1.5584 & 20 \\
MQT & 1 & 64 & 0.1299 & 20 \\
MQS & 2 & 16 & 0.2597 & 20 \\
MQS & 4 & 8 & 0.5195 & 20 \\
MQT & 8 & 32 & 1.0390 & 20 \\
MQTL & 1 & 56 & 0.1299 & 20 \\
MQTL & 2 & 8 & 0.2597 & 20 \\
MQTL & 6 & 8 & 0.7792 & 20 \\
\hline Total & & $\mathbf{3 5 2}$ & $\mathbf{2 . 4 1 1 4 5}$ & \\
\hline
\end{tabular}

Table 7.12: Quench rate of the LHC corrector circuits.

A false quench will also have different consequences depending on the detector. In this case, the differences are not from the protection strategy point of view, but from the function of the corrector in the machine. It has been assumed that the spurious signal from a corrector circuit placed in the arc will have the same effect than a false quench in the main magnets (i.e. five-hour shutdown). On the other hand, a quench in the insertion area will require shorter cooldown times, hence the unscheduled downtime can be reduced to just one hour.

The protection strategies and failure costs for the different families of LHC correctors are listed in Table 7.13.

Since the detection topology must be the same for all the corrector families an independent study for each group with common failure cost may not be made. A solution could be to optimise all the detectors under the worst assumptions, but it would be a too conservative solution far from the optimal one.

The average FQ costs have been evaluated according to the number of circuits. The MQ costs are averaged with respect to the expected quenches per circuit.

$$
\begin{gathered}
\overline{C^{F Q}}=\frac{\sum_{i} C_{i}^{F Q} n_{i}}{\sum_{i} n_{i}} \\
\overline{C^{M Q}}=\frac{\sum_{i} C_{i}^{M Q} \mu_{i} n_{i}}{\sum_{i} \mu_{i} n_{i}}
\end{gathered}
$$

where $C_{i}^{F Q}$ and $C_{i}^{M Q}$ are the cost of one false and missed quench respectively in one kind of circuit $i, n_{i}$ the number of circuits $i$ in the machine and $\mu_{i}$ the quenches expected for that family. 


\begin{tabular}{lcccc} 
& $\begin{array}{c}\text { Parallel } \\
\text { Resistor }\end{array}$ & $\begin{array}{c}\text { Energy } \\
\text { Extraction }\end{array}$ & $\begin{array}{c}\text { FQ } \\
\text { Cost }[\mathrm{h}]\end{array}$ & $\begin{array}{c}\text { MQ } \\
\text { Cost }[\mathrm{h}]\end{array}$ \\
\hline MCS & YES & YES & 5 & 672 \\
MCD & NO & YES & 5 & 672 \\
MCO & NO & PS & 5 & 67.2 \\
MO & NO & YES & 5 & 67.2 \\
MSS & YES & PS & 5 & 67.2 \\
MS & YES & YES & 5 & 672 \\
MQT & NO & PS & 1 & 67.2 \\
MQS & NO & PS & 1 & 67.2 \\
MQT & YES & YES & 1 & 672 \\
MQTL & NO & PS & 1 & 67.2 \\
MQTL & YES & YES & 1 & 672 \\
\hline
\end{tabular}

Table 7.13: Failure cost of the LHC corrector circuits.

Eqs. 7.6 and 7.7 give an average downtime after a false quench of $2.81 \mathrm{~h}$ and $538 \mathrm{~h}$ for a missed quench.

\subsubsection{Detector Design}

The corrector magnets are not provided with quench detectors for each magnet. Instead, a single detector monitors the status of the whole circuit.

A differential voltage is picked up at the cold ends of the superconducting current leads. The detector is equipped with a dedicated current sensor, which enables it to measure the current and to calculate the derivative. The hardware is based on a digital signal processor, which treats the incoming signals and computes the resistive voltage drop using the preloaded inductance tables thus it provides active compensation of the inductive voltage.

The electronic board will be like the DQQDI and DQQDT detectors with the only difference of the LEM-HAS current sensor with a failure rate $\lambda=7.14 \cdot 10^{-7} h^{-1}$.

This sensor is only used by the detector while the magnet chain is being ramped, since once the current reaches the nominal plateau, the differential voltage seen by the detector will correspond to the resistive voltage in the circuit. Hence, a failure of the sensor will avoid the compensation of the inductive voltage and generate a false quench.

\subsubsection{Studied Topologies}

The analysed detection schemes are the same than for the insertion and inner triplet detectors (see Fig. 7.18). The reliability values after 20 years for each topology are listed in Table 7.3.3. Monthly coherency checks and yearly quench tests are considered.

The best topologies from the MQ reliability point of view (1oo2, 2003 and 2003 with redundant voting part) have been analysed for a broad quench rate range. The maximum number of missed quenches (95\% confidence level) expected after 20 years with this maintenance strategy are shown in Figure 7.20. 


\begin{tabular}{l|cc|cc|cc|c} 
& \multicolumn{2}{|c|}{ UM } & \multicolumn{2}{c|}{ FQ } & \multicolumn{2}{c}{ MQ } & Events \\
Scheme & Rel. Time [d] \\
\hline 1oo1-1TMS & 0.7412 & $99-156$ & 0.5565 & $227-352$ & 0.9897 & $1-6$ & 112 \\
1oo2-1TMS & 0.9931 & $1-4$ & 0.5223 & $263-403$ & 0.9998 & 0 & 39 \\
2oo2-2TMS & 0.5480 & $236-365$ & 0.9362 & $20-37$ & 0.97939 & $4-12$ & 182 \\
2oo3-3TMS & 0.9815 & $3-11$ & 0.9187 & $22-41$ & 0.9995 & $0-1$ & 15 \\
2oo4-4TMS & 0.9870 & $2-7$ & 0.9029 & $28-50$ & 0.9996 & $0-1$ & 16 \\
2oo2or2oo2-4TMS & 0.9826 & $3-10$ & 0.9190 & $22-41$ & 0.9996 & $0-1$ & 27 \\
1ooand1oo2-2TMS & 0.9862 & $2-9$ & 0.9174 & $23-42$ & 0.9996 & $0-1$ & 27 \\
\hline
\end{tabular}

Table 7.14: DQQDG reliability and expected failures (95\% confidence level) after 20 years with monthly CT and yearly QT.

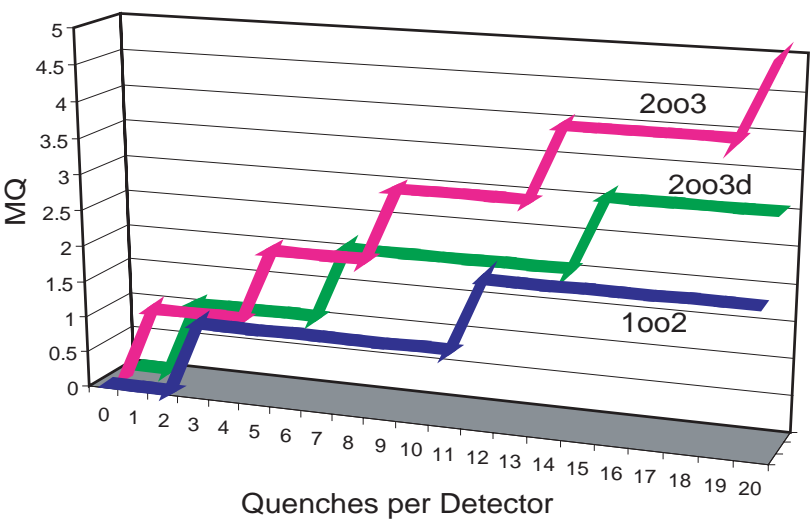

Figure 7.20: Maximum number of missed quenches (95\% confidence) after 20 years in a DQQDG detector in front of the the total expected quenches per detector (monthly CT and yearly QT).

The quench checks on this detectors can be carried out without affecting the coil and the cryogenics since they are not provided with quench heaters. This makes much easier to increase the QT frequency from yearly to monthly. In this way the missed quench probability almost vanishes for the 1oo2 topology. This good MQ reliability together with space reasons make 1002 the chosen solution.

The FQ performance improves if redundant power supplies in parallel are installed. The individual FQ reliability increases from $52 \%$ to $70 \%$ (263-403 to $120-187$ false quenches with a 95\% confidence level). Despite the improvement, the number of false quenches stays remarkably high due to the analog and digital parts, which, like for the DQQDT and DQQDI detectors, do not have redundancy at all (see Fig. 7.21). The redundant powering is recommended if the coherency checks are properly modified in order to monitor the state of each power supply.

Since the current sensor will be a source of false quenches only, the reliability of the system will no improve if it is doubled. Hence, a unique sensor will be used for both channels. 

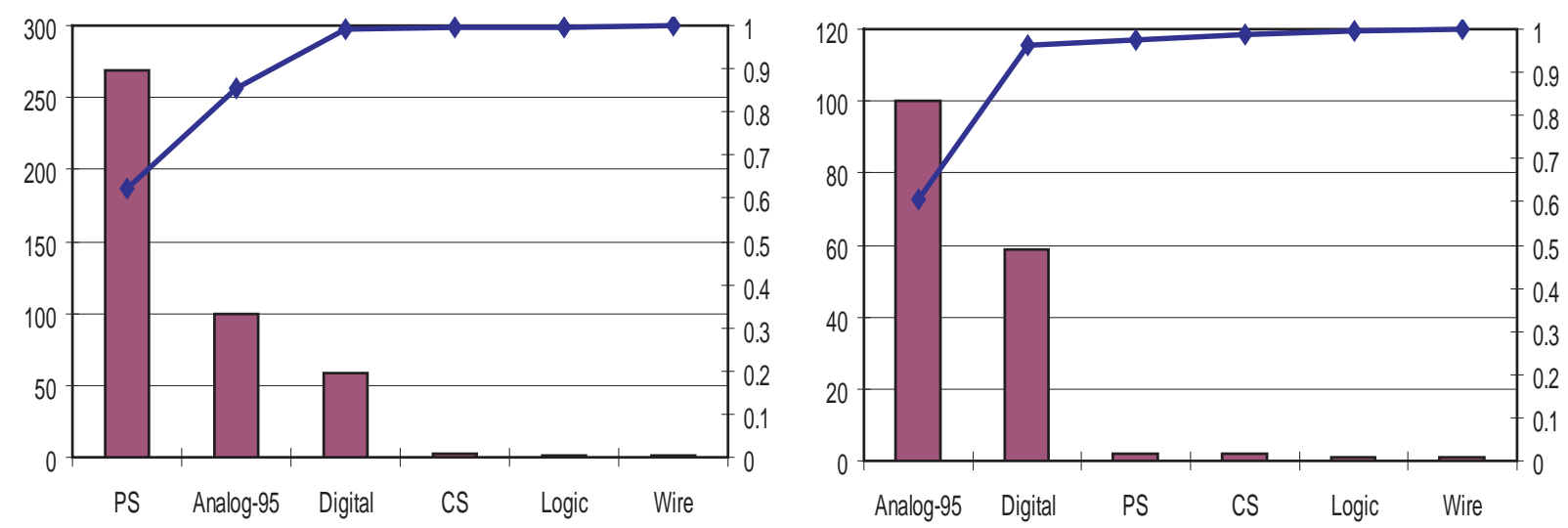

Figure 7.21: Pareto analysis of false quenches in DQQDG detectors for 1002 detection scheme without redundant powering (left) and with shared power supplies (right). Monthly CT and yearly QT have been considered.

\subsection{Superconducting Current Lead Quench Detectors (DQQDC)}

Each one of the 1,198 HTS-based superconducting current leads is protected by a detector that supervises the resistive and superconducting part of the lead independently.

Quenches in current leads are very unlikely, hence it is rather difficult to estimate their occurrence rate. Any fixed quench rate prediction would no be very confident, therefore the analysis must be completed with a broad sensitivity analysis.

\subsubsection{Detector Design}

The system must be able to identify a voltage development above $3 \mathrm{mV}$ during 1 second. The microprocessor will need an auxiliary SRAM memory in order to evaluate the signal for such period. Figure 7.22 represents a one-detector channel layout.

\subsubsection{Reliability Data of the Components}

Table 7.15 lists the nominal values for the 1198 DQQDC detectors. The same powering characteristics than for DQQDI and DQQDT detectors are considered.

\subsubsection{Studied Topologies}

The analysed detection schemes are the same than the ones studied above (see Fig. 7.18). The reliability performance after 20 years for each topology are listed in Table 7.4.3. Mothly coherency checks and yearly quench tests are considered. It has been assumed that every current lead will 


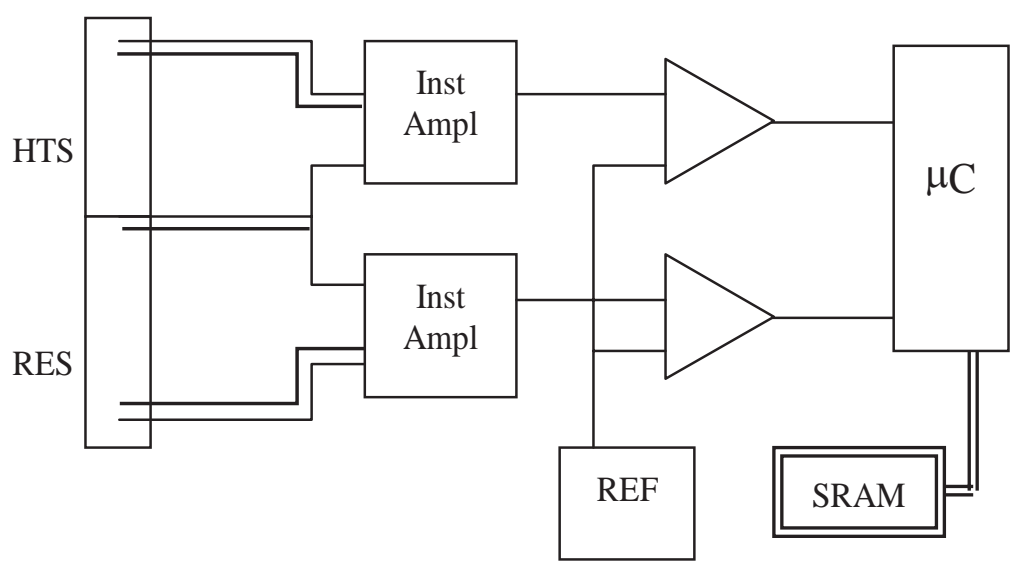

Figure 7.22: One-channel DQQDC quench detector.

\begin{tabular}{lccc}
\multicolumn{1}{c}{ Device } & Name & MTBF & Failure Rate \\
\hline Instrumentation Amplifier $^{8}$ & INA141P & $1447329 h$ & $6.90927 \cdot 10^{-7} h^{-1}$ \\
Operational Amplifier $^{8}$ & INA105AM & $5644583 h$ & $1.77161 \cdot 10^{-7} h^{-1}$ \\
Voltage Reference $^{8}$ & AD780BN & $4341992 h$ & $2.30309 \cdot 10^{-7} h^{-1}$ \\
Microcontroller $^{9}$ & ADUC816 & $1628131 h$ & $6.14201 \cdot 10^{-7} h^{-1}$ \\
Memory $^{9}$ & SRAM 32kx8 & $8918219 h$ & $1.1213 \cdot 10^{-7} h^{-1}$ \\
Power Supply (AC-DC) $^{10}$ & Traco TMS1505C & $250000 h$ & $4.0 \cdot 10^{-6} h^{-1}$ \\
Power Supply (DC-DC) $^{10}$ & Traco TMA & $>2000000 h$ & $5.0 \cdot 10^{-7} h^{-1}$ \\
Wire $^{11}$ & & $26371308 h$ & $3.792 \cdot 10^{-8} h^{-1}$ \\
Logic $^{9}$ & CMOS gates & $0.021+0.0014 \cdot(\# \text { pins })^{1.08} h$ \\
\hline
\end{tabular}

Table 7.15: Failure rate data for DQQDC components.

quench once during the 20 operational years. This is a very pessimistic and arbitrary value, hence a wide sensitivity analysis has been carried out.

The expected maximum number of missed quenches in function of the current lead quench rate is plotted in Figure 7.23 for the two best detection schemes.

Like for the two detectors previously analised, the best MQ reliability is shown by the 1002 topology. Again, its simplicity and adaptability to the space constrains make it the chosen solution.

The two boards can be, like for the DQQDI, DQQDT, and DQQDG detectors, powered by two shared power supplies. The coherency tests as described in the local quench detectors will be useless in this configuration unless they are modified in order to monitor the status of each power supply. Monthly coherency checks will then decrease the number of false quenches from 269-312 to $132-165$ (95\% confidence level).

\footnotetext{
${ }^{8}$ Data from manufacturer. Arrhenius formula used with $1 \mathrm{eV}$ activation energy (see Appendix B).

${ }^{9}$ Data from MIL-HDBK-217F. $G_{B}$ :fix grounded.

${ }^{10}$ Data from Manufacturer

${ }^{11}$ Data from MIL-HDBK-217F. $G_{B}$ [44]. Four soldering per wire (one at cold and three at warm), clip terminator.
} 


\begin{tabular}{|c|c|c|c|c|c|c|c|}
\hline \multirow[b]{2}{*}{ Scheme } & \multicolumn{2}{|c|}{ UM } & \multicolumn{2}{|c|}{ FQ } & \multicolumn{2}{|c|}{ MQ } & \multirow[b]{2}{*}{ Av. Time $[\mathrm{d}]$} \\
\hline & Rel. & Events & Rel. & Events & Rel. & Events & \\
\hline 10o1-1TMS & 0.7899 & $229-270$ & 0.6140 & $242-284$ & 0.9764 & $20-35$ & 824 \\
\hline 10o2-1TMS & 0.9947 & $3-10$ & 0.5740 & $269-312$ & 0.9996 & $0-1$ & 74 \\
\hline 2oo2-2TMS & 0.6224 & $425-474$ & 0.9944 & $1-6$ & 0.9528 & $45-66$ & 1554 \\
\hline 2oo3-3TMS & 0.9918 & $5-14$ & 0.9870 & $4-13$ & 0.9994 & $0-2$ & 29 \\
\hline 2004-4TMS & 0.9872 & $9-20$ & 0.9744 & $11-23$ & 0.9987 & $0-3$ & 45 \\
\hline 2oo2or2oo2-4TMS & 0.9891 & $7-18$ & 0.9875 & $4-12$ & 0.9993 & $0-2$ & 30 \\
\hline 1ooand1oo2-2TMS & 0.9895 & $7-17$ & 0.9884 & $4-12$ & 0.9884 & $0-2$ & 30 \\
\hline
\end{tabular}

Table 7.16: DQQDC reliability and expected failures (95\% confidence level) after 20 year with montly CT and yearly QT.

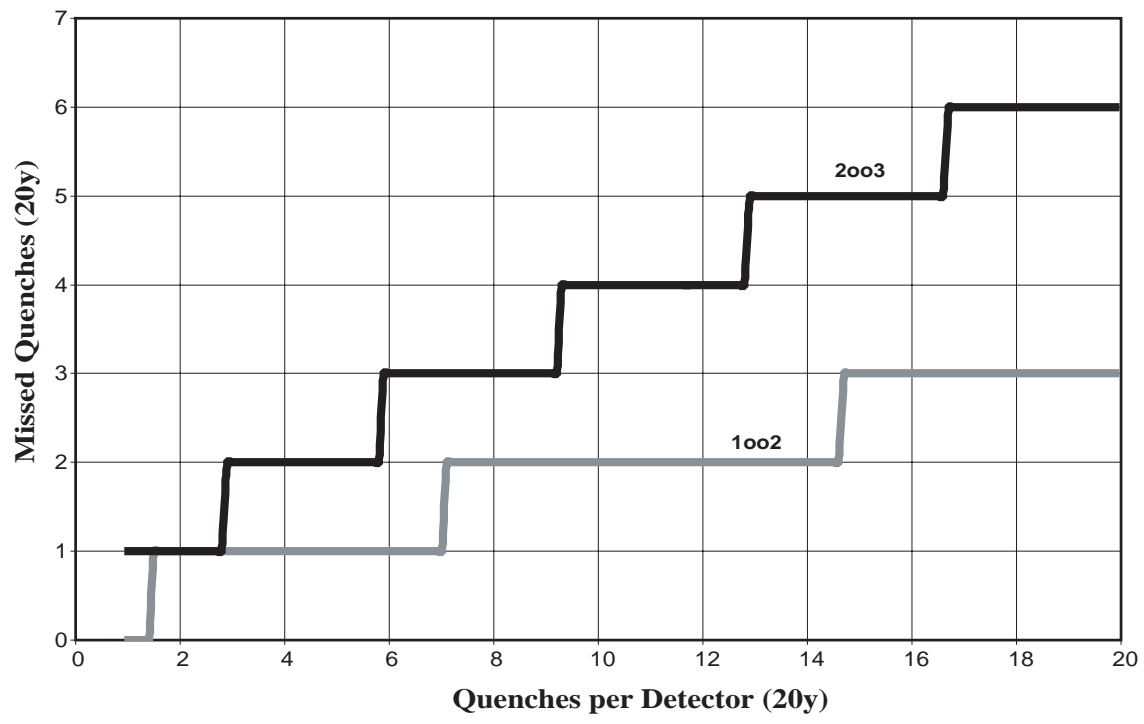

Figure 7.23: Maximum number of missed quenches ( $95 \%$ confidence) after 20 years in a DQQDC detector in function of the total expected quenches per detector (Monthly CT and yearly QT). 



\section{Chapter 8}

\section{Reliability of the LHC Quench Heater Power Supplies}

\subsection{The LHC Quench Heater Power Supplies (DQHDS)}

The quench heater power supplies were introduced in Chapter 2. They are the devices in charge of energising the heater strips of the main dipole and quadrupole magnets.

In Chapter 6, it was demonstrated that high field heaters were enough to redundantly ensure the proper quench propagation. This chapter analyses the reliability of the power supplies connected to the high field heaters.

The main dipoles are protected by four power supplies that are simultaneously fired after a quench is detected. Each power supply energises a couple of quench heaters (see Fig. 8.1). The quench heaters of the arc quadrupoles are fed by only two power supplies. Each one powers two heaters of each aperture (see Fig. 8.1). In this way, although the apertures are powered by different circuits, both will be fired after a signal of any of the two quench detectors.

The $6 \mathrm{kA}$ quadrupoles in the insertion region are also protected with quench heaters. All the circuits, except those formed by MQY magnets, are protected by two power supplies per magnet. The MQY circuits will have four power supplies per magnet. In all the cases, and in contrast to the arc magnets, all the power supplies of the circuit will be fired after one of its magnets quenches.

Two out of the four power supplies have to be discharged in order to safely propagate the quench in the dipole magnets, and at least one for the rest.
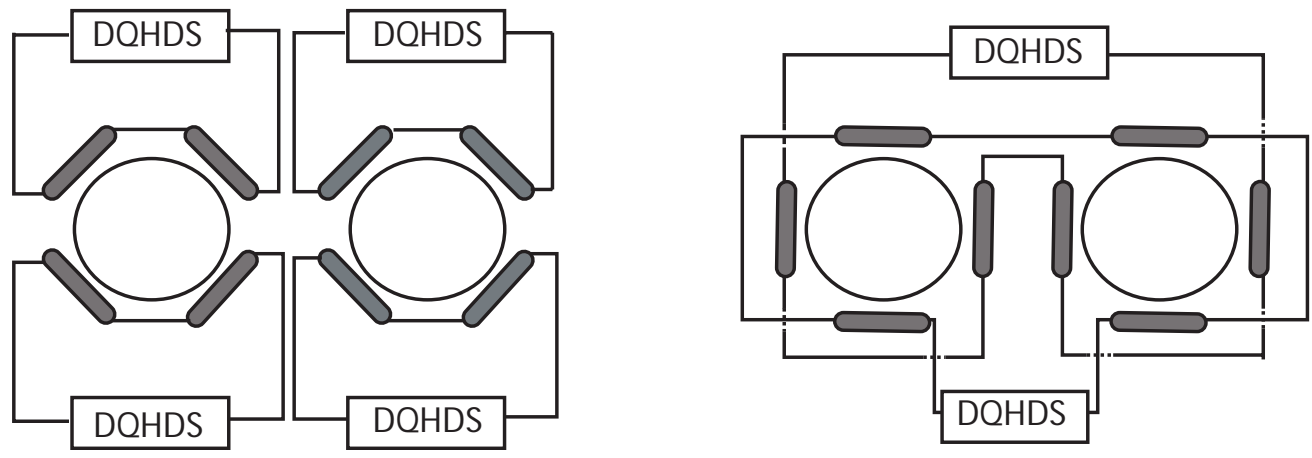

Figure 8.1: Quench heater circuits for the LHC main dipole (left) and quadrupole (right). 


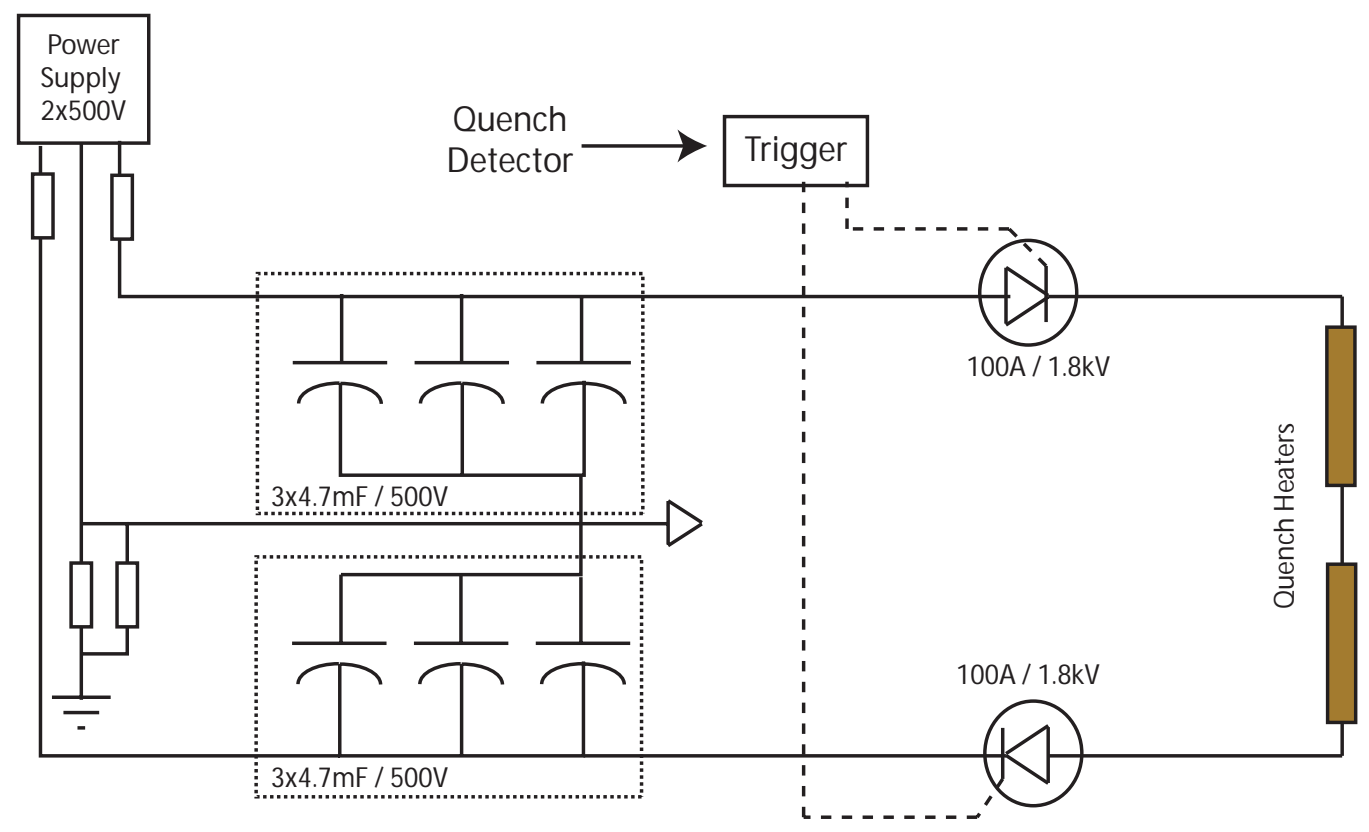

Figure 8.2: Electrical design of the LHC quench heater power supplies.

\subsubsection{Redundant Design}

The quench heater power supplies have the same design for all the magnet families. They contain two branches of three aluminium electrolytic capacitors connected in parallel. The capacitors are discharged on the strips by triggering two thyristors connected in series in order to reduce the unwanted discharge probability (see Fig. 8.2).

Besides the power part, the heater power supply units require an interface to the Acquisition and Monitor Controllers for maintenance and interlock purposes.

Electrolytic capacitors may fail due to different reasons. One of the main causes of failure is the eventual drying out of the electrolyte. This results in a decrease of capacitance, an increase of the dissipation factor or, at worst, an open circuit. Since short circuits in electrolytic capacitors have become very uncommon, the only capacitor failure mode considered in this study is the open circuit. These capacitors are suitable for applications in which high reliability is required for long lifetimes (10-20 years). They are not suitable for circuits in which charge and discharge are frequently repeated. The capacitors [29] will be operated at $7 \% \pm 5 \%$ of their rated voltage leading to an increased lifetime and a lower leakage current.

In order to evaluate the redundant level of the capacitors in one single quench heater power supply a conservative approach has been chosen. In [18] it was set $V_{\min }=700 \mathrm{~V}$ as the minimum voltage across the capacitors needed for properly energising the two heater strips. In order to keep a safety margin, the minimum voltage acceptable for power permit will be $800 \mathrm{~V}$. The maximum energy dissipated in both quench heaters will be

$$
E_{Q H \max }=\frac{1}{2} C V^{2}
$$

$V_{\min }$ was computed considering all the capacitors available. The minimum HPS capacitance $C_{p p}$ needed at the minimum voltage accepted by the operator before the power permit $V_{p p}$ will be given by 


$$
\frac{C_{\text {nom }}}{C_{\text {min }}}=\frac{V_{p p}^{2}}{V_{\text {min }}^{2}}=\left(\frac{800}{700}\right)^{2} \Rightarrow C \geq 0.765 C_{\text {nom }}
$$

Where $C_{n o m}$ is the total nominal capacitance with all the capacitors in the two branches available (i.e. 3-3). Using the nomenclature (m-n) where $m$ and $n$ are the number of capacitors available in the first and second branch respectively, the total capacitance at any moment $t$ will be

$$
C_{n, m}=\frac{m \cdot n}{m+n} C
$$

Therefore, Eq. 8.2 sets 3-3, 2-3 and 3-2 (i.e. at least 5 capacitors available) as the only safe states if $V_{\text {crate }}>800 \mathrm{~V}$. In these cases the energy is above the minimum threshold for quenching the coil and, since the total resistance remains constant (11.2 $\Omega$ [18]), the time constant is lower than the nominal. The combination 2-2 would also work at the nominal capacitor voltage $(900 \mathrm{~V})$ but then the model would not be conservative.

The thyristors SCR foreseen for the power supplies are intentionally overrated in order to cope with irradiation induced changes of the device parameters. They are in series redundancy in order to avoid false triggers, therefore both thyristors must be triggered for discharging the capacitors. Radiation and aging tests (see Appendix A) have shown that the only failure mode of the thyristors under LHC environmental conditions is the impossibility to be triggered. The spurious triggers will not be, therefore, considered in this study.

Failure data from MIL-HDBK-217F for electrolytic aluminium capacitors and the two types of SCR thyristors used are summarised in Table 8.1.

\begin{tabular}{lrrc}
\multicolumn{2}{c}{ Device } & MTBF & Failure Rate \\
\hline Capacitors & Electrolytic & $1108419 h$ & $0.902186910^{-6} h^{-1}$ \\
Thyristors & Semicron & $3563919 h$ & $0.2805910^{-6} h^{-1}$ \\
& Westcode & $3688404 h$ & $0.2711210^{-6} h^{-1}$ \\
\hline
\end{tabular}

Table 8.1: Component reliability data from MIL-HDBK-217F.

\subsubsection{Failure Modes}

Within the scope of this analysis a heater power supply is considered to be damaged (which does not mean necessarily unavailable) when one or more capacitors are at open circuit or any of the thyristors is unable to be fired. We have, therefore, the following failure scenarios:

- HPS Unavailable: More than one capacitor are at open circuit or a thyristor is blocked. The magnet will be still protected if the other power supplies work properly.

- Magnet Unprotected: This dangerous failure occurs when two there are not enough HPS available in a magnet to properly protect it.

- Lost Magnet: This is the main failure. The superconducting magnet quenches when it is unprotected. 


\subsubsection{Maintainability}

In order to increase the availability of the quench heater power supplies, two different checks are foreseen:

- Voltage Test (VT): The voltage across the power supplies is monitored continuously. If it is below $V_{p p}$ the power permit is not given. Since the only possible failure of the capacitors considered in this study (pessimistic approach) is the open circuit, this test will show that a HPS is unavailable only when the three capacitors of a branch fail. The test will be also blind to problems in the thyristors.

- Discharge Test (DT): A simulated quench signal is sent from the control room for checking the quench detectors and the heater power supplies at the same time. The discharge and charge of the power supplies is monitored with the same channel used for the voltage test. This test shows the complete status of the device: thyristor not fired and number of capacitors damaged. Obviously this test can only be performed when the magnets are cold and not powered.

The natural quenches and the post-mortem data can be considered as discharge tests. The different repair policies after one or more damaged power supplies have been found are:

- Policy 0: Post-mortem ignored. Checks and repairs every DT test.

- Policy 1: Post-mortem considered but not repair done until next scheduled technical stop.

- p1_Xhps: Repair after $X$ heater power supplies are damaged.

- p1_Xm: Repair after $X$ months.

- Policy 2: Post-mortem considered and system fixed before power permit.

\subsubsection{Power Supply Banks and Quench Rate}

The quench heater power supplies that are simultaneously fired by the same quench detector signal are called a power supply bank. The discharges of a bank resulting from a quench can be computed from the quench rates set in Chapter 7: 24 quenches per operational week for the local quench detectors.

This value was assumed for ring dipoles and quadrupoles, the two detectors of each quadrupole were considered independent. Since the HPS in the main quadrupoles are shared by both apertures, one HPS will be fired by two independent quench detectors, hence the quench rate for the ring quadrupoles will be twice the quench rate for the main dipoles. In order to compute all the LHC quadrupoles together the quench rate for both ring and insertion quadrupoles has been computed using the quench data of Chapter 7.

The quench rates for the power supplies of the different quadrupole families have been averaged. The study is carried out independently for the dipoles and the quadrupoles.

In Table 8.1.4 are listed the different magnet families and the number of quenches expected per power supply bank. 


\begin{tabular}{lcccccc} 
& $\begin{array}{c}\text { Magnets } \\
\text { per Circuit }\end{array}$ & $\begin{array}{c}\text { DQHDS } \\
\text { per Magnet }\end{array}$ & $\begin{array}{c}\text { PS } \\
\text { Banks }\end{array}$ & $\begin{array}{c}\text { Detectors } \\
\text { per PS Bank }\end{array}$ & $\begin{array}{c}\text { Quenches per } \\
\text { PS Bank (20 y) }\end{array}$ & $\begin{array}{c}\text { Av. Quenches } \\
\text { per Bank }\end{array}$ \\
\hline MB & 154 & 4 & 1232 & 1 & 7.242063 & 7.2421 \\
MQ 49 & 2 & 392 & 2 & 14.4841 & & \\
MQM+MQMC & 2 & 2 & 4 & 2 & 20 & \\
MQM & 1 & 2 & 4 & 2 & 10 & \\
MQM+MQM & 2 & 2 & 28 & 2 & 20 & \\
MQML & 1 & 2 & 64 & 2 & 10 & \\
MQY & 1 & 4 & 16 & 2 & 10 & \\
MQY+MQY & 2 & 4 & 12 & 1 & 57 & \\
MQXA & 2 & 2 & 8 & 2 & 60 & \\
MQXB & 2 & 2 & 8 & 2 & & \\
& & 536 & & &
\end{tabular}

Table 8.2: LHC quench power supplies and quench rates.

\subsection{Reliability Models}

In order to make the model more understandable the two different checks are treated independently. Since the possible false quenches are not considered, the multi-state protection system model used for the quench detectors can be simplified at its first stage (before computation of random walk) to a Markovian model. The simple topology allows an analytical solution of the reliability equations.

\subsubsection{Voltage Test}

Figure 8.3 represents part of the system Markov diagrams used to implement the reliability model considering only voltage tests.

If we define $P_{i, j}(T)$ as the probability of having the configuration $i, j$ at the present time $T$, the expected availability of a single power supply due to the capacitors at a certain time, $E \mathcal{A}_{c}(T)$, will follow Eq. 8.4.

$$
E \mathcal{A}_{c}(T)=\sum_{i} \sum_{j} P_{i, j}(T), \forall i, j \mid H P S \text { available }
$$

with

$$
\begin{aligned}
P_{p 1, p 2}(T)= & \sum_{i=p 1}^{C} \sum_{j=p 2}^{C} \mathcal{B}\left(\begin{array}{c}
i \\
p 1
\end{array}\right)_{t} \mathcal{B}\left(\begin{array}{c}
j \\
p 2
\end{array}\right)_{t} P_{i, j}^{N-1}+ \\
& \mathcal{B}\left(\begin{array}{c}
C \\
p 1
\end{array}\right)_{t} \mathcal{B}\left(\begin{array}{c}
C \\
p 2
\end{array}\right)_{t} P_{\text {det }}^{N-1}, \forall p 1, p 2 \neq 0
\end{aligned}
$$




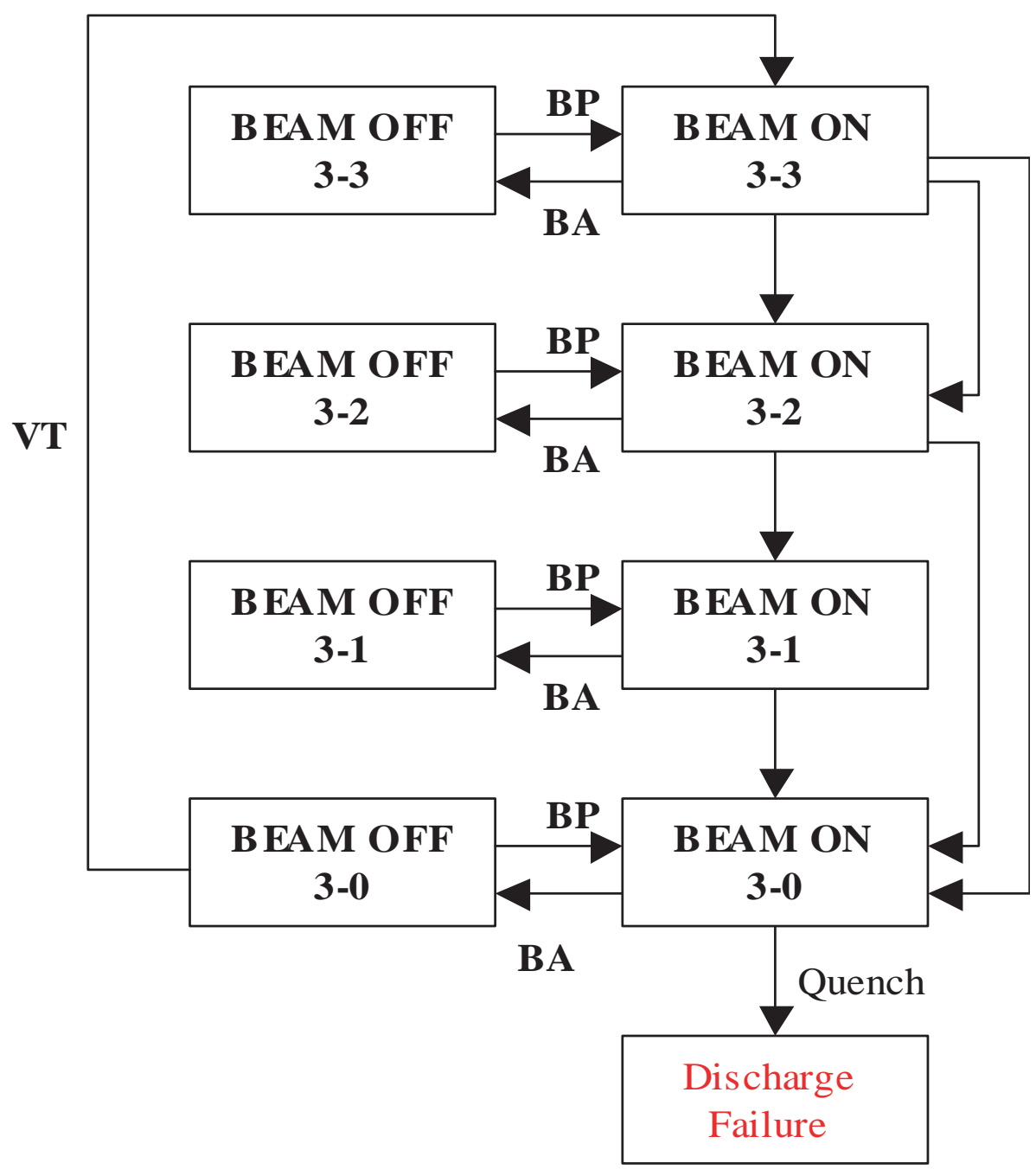

Figure 8.3: Markov diagrams (state-space) for one branch with voltage checks (VT); BP: Beam Permit, BA: Beam abort.

$$
\begin{aligned}
& P_{p 1,0}(T)= \sum_{i=p 1}^{C} \sum_{j=1}^{C} \mathcal{B}\left(\begin{array}{c}
i \\
p 1
\end{array}\right)_{t} \mathcal{B}\left(\begin{array}{c}
j \\
0
\end{array}\right)_{t} P_{i, j}^{N-1}+ \\
& \mathcal{B}\left(\begin{array}{c}
C \\
p 1
\end{array}\right)_{t} \mathcal{B}\left(\begin{array}{c}
C \\
0
\end{array}\right)_{t} P_{d e t}^{N-1}, \forall p 1 \neq 0 \\
& P_{0,0}(T)=\sum_{i=1}^{C} \sum_{j=1}^{C} \mathcal{B}\left(\begin{array}{c}
i \\
0
\end{array}\right)_{t} \mathcal{B}\left(\begin{array}{l}
j \\
0
\end{array}\right)_{t} P_{i, j}^{N-1}+\mathcal{B}\left(\begin{array}{c}
C \\
0
\end{array}\right)_{t} \mathcal{B}\left(\begin{array}{c}
C \\
0
\end{array}\right)_{t} P_{\text {det }}^{N-1}
\end{aligned}
$$

where $\mathcal{B}\left(\begin{array}{c}m \\ n\end{array}\right)_{t}$ is the value of the Bernoulli distribution for $n$ events in $m$ trials with a probability defined by the failure distribution of each capacitor from the last beam permit. Since the hypothesis of memoryless components is assumed, the failure rate $\lambda$ is constant (see Table 8.1). 
The component failure probability will, therefore, follow an exponential distribution. $C$ is the number of capacitor per branch, $N$ the number of completed runs and $P_{i, j}^{N-1}$ the probability of having the configuration $i-j$ at the end of the last run. The term $P_{d e t}^{N-1}$ includes the voltage check as the possibility of having detected a charge failure (one whole branch failed) in the last beam abort. It is given by the expression:

$$
\begin{aligned}
P_{\text {det }}^{N}= & 2 \sum_{i=1}^{C} \sum_{j=1}^{C} \sum_{k=1}^{j} \mathcal{B}\left(\begin{array}{l}
i \\
0
\end{array}\right)_{t} \mathcal{B}\left(\begin{array}{l}
j \\
k
\end{array}\right)_{t} P_{i, j}^{N-1}+ \\
& \sum_{i=1}^{C} \sum_{j=1}^{C} \mathcal{B}\left(\begin{array}{l}
i \\
0
\end{array}\right)_{t} \mathcal{B}\left(\begin{array}{l}
j \\
0
\end{array}\right)_{t} P_{i, j}^{N-1}+ \\
& 2 \sum_{i=1}^{C} \mathcal{B}\left(\begin{array}{c}
C \\
0
\end{array}\right)_{t} \mathcal{B}\left(\begin{array}{c}
C \\
i
\end{array}\right)_{t} P_{\text {det }}^{N-1}+ \\
& \mathcal{B}\left(\begin{array}{c}
C \\
0
\end{array}\right)_{t} \mathcal{B}\left(\begin{array}{c}
C \\
0
\end{array}\right)_{t} P_{\text {det }}^{N-1}
\end{aligned}
$$

The Voltage Check does not affect to the thyristor reliability, which can be modelled with the Bayesian equation for two independent variables following an identical exponential distribution. The system availability term related to the thyristors $E \mathcal{A}_{t}(T)$ will be:

$$
E \mathcal{A}_{t}(T)=e^{-\lambda_{t} T}
$$

and the expected availability of a single HPS with only voltage test performed will be defined by:

$$
E \mathcal{A}_{h p s}(T)=E \mathcal{A}_{c}(T) E \mathcal{A}_{t}(T)
$$

The availability of the whole HPS bank for one dipole can be expressed as the Bernoulli distribution for at least three successes (one for the quadrupoles) where a success is defined as the availability of the HPS after four trials (two for the quadrupoles). The probability of success is given by $E \mathcal{A}_{h p s}(T)$. The probability of a dipole to be unprotected at T $P_{U M}(T)$ will be then:

$$
P_{U M}(T)=1-\sum_{i=3}^{4} \frac{4 !}{i !(4-i) !} \mathcal{A}_{h p s}(T)^{i}\left(1-\mathcal{A}_{h p s}(T)\right)^{4-i}
$$

The Figure 8.4 shows the unprotected magnet reliability with and without daily voltage tests. The values have been computed considering the effect of the thyristor failures and without it. It can be seen that the effect of the voltage checks is negligible.

All the expressions above make reference to the reliability of a single magnet. In order to compute the total number of unprotected or lost magnets in the machine, RESQP uses the Bernoulli distribution. The availability is an instantaneous value, for computing the failures after a certain time the reliability must be used instead. Nevertheless, seen the little influence of the voltage test, the expected availability is a good approximation of the reliability. In Figure 8.5 the expected unprotected magnets have been plotted for the same cases than before. 


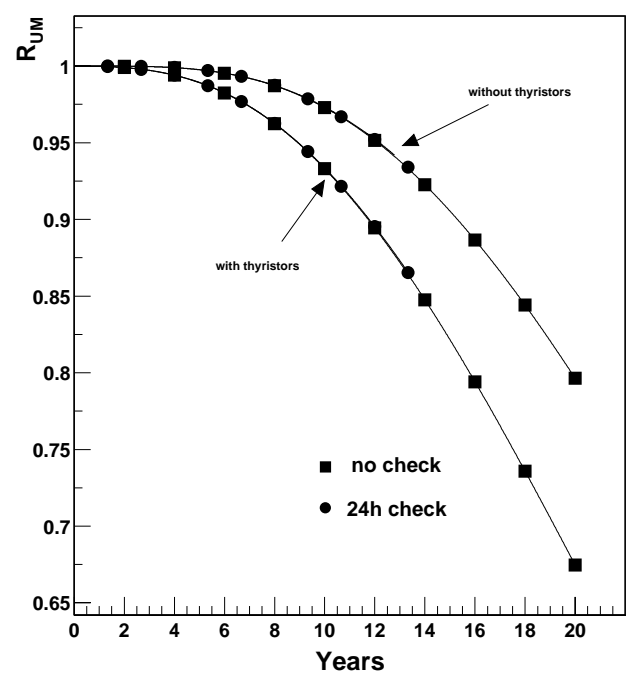

Figure 8.4: Unprotected magnet reliability for a single dipole magnet.

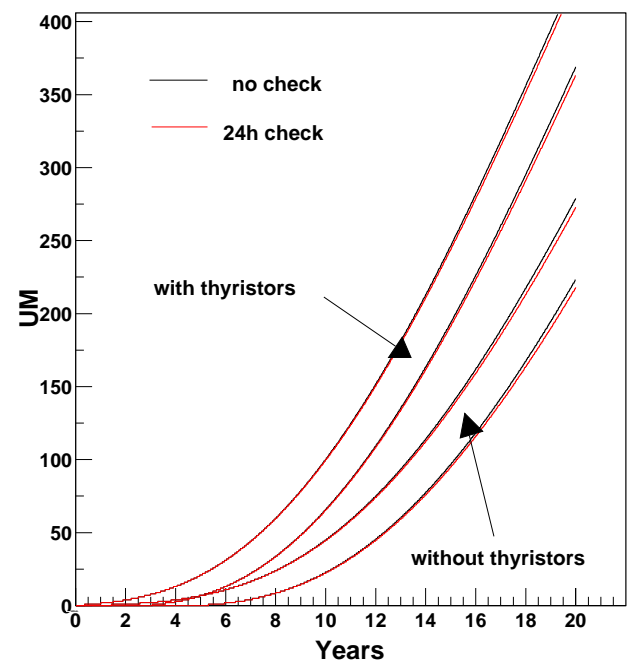

Figure 8.5: Number of unprotected dipoles with a 95\% confidence level.

The probability of a magnet to be unprotected at least once in 20 years is $32.2 \%$ with daily tests and 32.54\% without them. The maintenance of the system must be, therefore, improved with another kind of check.

\subsubsection{Discharge Test}

This check provides the exact thyristor and capacitor status. We consider that after the check all the power supplies are as good as new. Figure 8.6 represents a part of the system Markov diagram used for the reliability model with discharge tests. Two repair policies are represented: post-mortem data ignored (i) or considered (ii). 


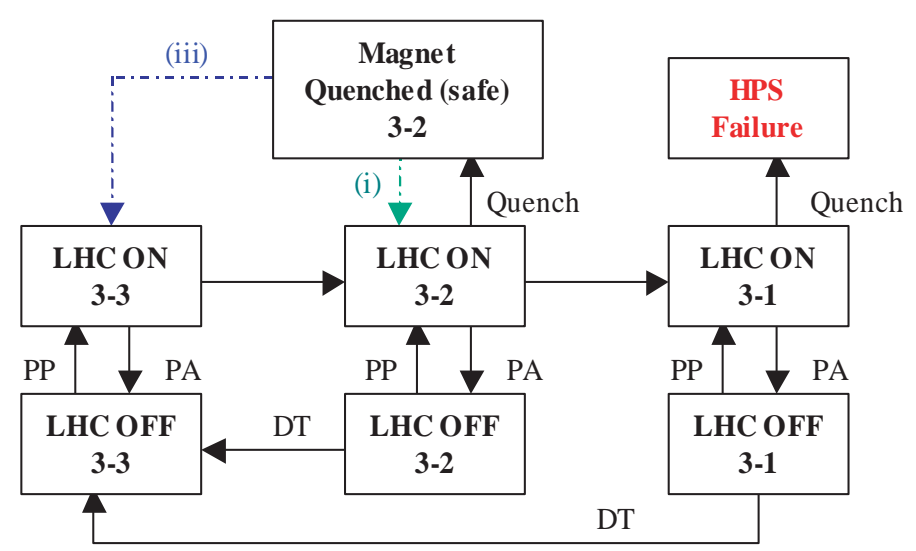

Figure 8.6: Markov diagram with discharge test (PP:Power Permit, PA:Power Abort, DT:Discharge Test).

If the post-mortem information is ignored (policy 0 ) the HPS reliability related to the capacitors can be modelled by Eq. 8.12. The time step between iterations $t_{i}$ is the time between discharge checks $t_{c}$.

$$
R_{c}(T)=\sum_{i} \sum_{j} P_{i, j}(T), \forall i, j \mid H P S \text { available }
$$

where $P_{i, j}(T)$ is defined by Eq. 8.13 with $R_{c}^{N-1}$ being the reliability of the capacitor part before last check was performed.

$$
P_{i, j}(T)=\mathcal{B}\left(\begin{array}{c}
C \\
i
\end{array}\right)_{t} \mathcal{B}\left(\begin{array}{c}
C \\
j
\end{array}\right)_{t} R_{c}^{N-1}
$$

If the post-mortem data is taken into account and repairs are done during the next scheduled short shutdown. Being $t_{q}$ the expected time between quenches, the iteration time has to follow

$$
t_{i}^{-1} \leq t_{c}^{-1}+t_{q}^{-1}
$$

and now $P_{i, j}(T)$ will be defined as

$$
P_{i, j}(T)=\left\{\begin{array}{cl}
\mathcal{B}\left(\begin{array}{c}
C \\
i
\end{array}\right)_{t} \mathcal{B}\left(\begin{array}{c}
C \\
j
\end{array}\right)_{t} R_{c}^{N-1} & \text {, if DT in last run } \\
\sum_{m=i}^{C} \sum_{n=j}^{C} \mathcal{B}\left(\begin{array}{c}
i \\
m
\end{array}\right)_{t} \mathcal{B}\left(\begin{array}{c}
j \\
n
\end{array}\right)_{t}, \text { if not }
\end{array}\right.
$$

Some variations of policy 1 would be repairing after a certain number of power supplies are failed or always after a fixed time period (e.g. monthly).

Finally, the repair can be done just after the failure is detected (policy 2). Eqs. 8.14 and 8.13 are used but now with $t_{i}$ like in Eq. 8.14.

The thyristor reliability $R_{t}(T)$ will follow for policy 0 and policy 2 . 


$$
R_{t}(T)=e^{-\lambda t_{i} N}
$$

where $N$ is the number of tests carried out. For policy 1 Eq. 8.16 is slightly modified

$$
R_{t}(T)=e^{-\lambda_{t}\left(t_{q}^{-1}+t_{c}^{-1}\right)^{-1} N} e^{-\lambda_{t} t}
$$

where $t$ is the time from last test or quench.

The lost magnet probability $P_{L M}(T)$ has been computed from the distribution of quenches after a magnet becomes unprotected and before next check. RESQP solves the problem using Random Walk and Renewal Theory. As it was explained in Chapter 5, the probability of failure after the magnet becomes unprotected, or lost magnet probability is

$$
P_{L M}(T)=1-e^{-\mu \int_{0}^{T}(1-R(\tau)) d \tau}
$$

where $\mu$ is the expected quench rate. Since the quench process follows a Poisson distribution, $\mu$ is a constant.

\subsection{Reliability Results}

In Figure 8.7 the thyristor and capacitor reliability terms of one HPS have been represented separately for the different maintenance strategies.

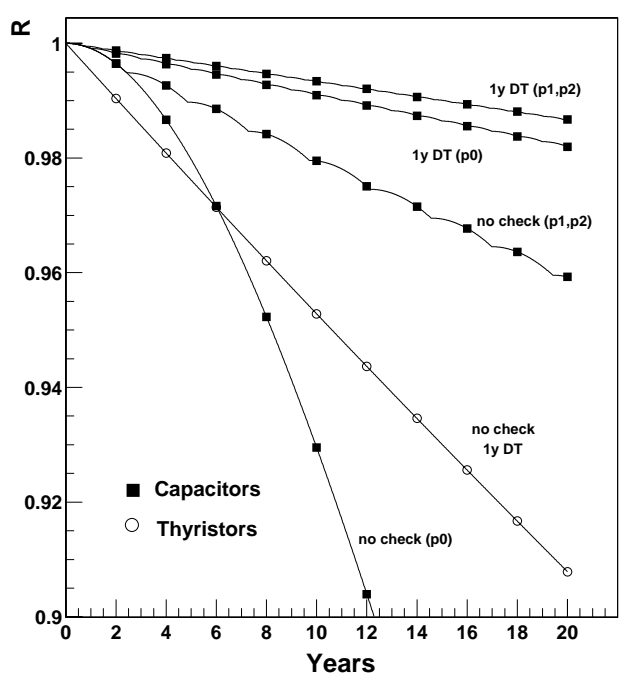

Figure 8.7: Capacitor and thyristor reliability terms for a single HPS and for different maintenance policies.

Several conclusions can be taken from these first results: 
- The thyristor reliability is not affected by the discharge tests. This doesn't mean that DT will not decrease failures due to this components, these tests will increase their availability and its effect will be noticed when computing the lost magnet probability. This is a demonstration that frequent checks can not improve the reliability of non redundant protection systems but only their availability.

- Post mortem information remarkably increases the capacitor reliability even when no discharge test is carried out.

- Yearly discharge tests are enough for increasing almost a $25 \%$ the reliability of every HPS after 20 years.

- Capacitors will be the main source of failures only if discharge tests are not carried out and post-mortem information ignored.

- Although there is a quantitative improvement between policy 0 and policy 1 , the difference is neglectable between this last one and policy 2 .

Figure 8.8 shows (left) the total heater power supply reliability and the unprotected magnet reliability (i.e. HPS bank reliability) for one dipole and (right) the unprotected and lost magnet reliabilities for all the LHC dipoles.
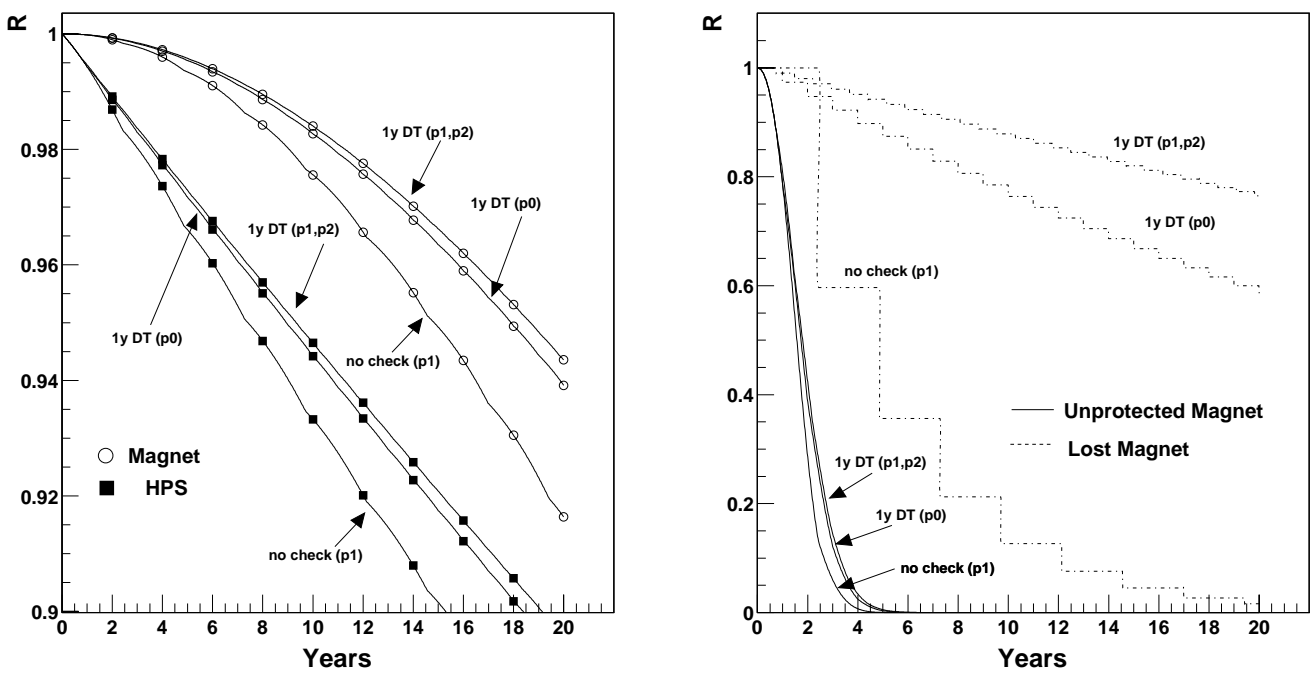

Figure 8.8: Left: Capacitor and thyristor reliability terms for a single HPS. Right: Unprotected magnet and lost magnet reliability for the 1232 LHC dipoles.

The number of lost HPS, unprotected magnets and lost magnets (95\% confidence level) after 20 years of LHC operation for the different maintenance policies (see Section 8.1.3)) are listed in Table 8.3 for 5110 operational yearly hours, 1232 banks for the dipole magnets and 536 for the quadrupoles.

Quadrupoles are properly protected if two discharge tests are carried out per year and postmortem information is taken into account. The risk of loosing a magnet is rather low for the dipoles if the same maintenance strategy is applied (below 8\%), nevertheless, if it is possible, 


\begin{tabular}{ll|cccc} 
& Policy & HPS & UM & LM & $R_{L M}$ \\
\hline no check p1 & $591-683$ & $84-122$ & $1-9$ & 0.016 \\
no check p2 & $577-669$ & $81-118$ & $0-8$ & 0.02685 \\
1y DT p0 & $492-578$ & $59-92$ & $0-2$ & 0.584029 \\
1y DT p1 6m & $479-564$ & $56-88$ & $0-2$ & 0.763899 \\
1y DT p1 & $472-556$ & $54-86$ & $0-2$ & 0.765017 \\
& 1y DT p2 & $458-541$ & $51-82$ & $0-2$ & 0.772645 \\
6m DT p0 & $454-537$ & $50-81$ & $0-1$ & 0.888074 \\
& 6m DT p1 & $448-530$ & $49-79$ & $0-1$ & 0.920444 \\
Dipoles & 1m DT p0 & $422-517$ & $45-78$ & 0 & 0.9972 \\
1m DT p1 & $410-500$ & $40-70$ & 0 & 0.9974 \\
\hline & no check p1 & $103-144$ & $2-13$ & $0-1$ & 0.857553 \\
no check p2 & $102-142$ & $2-12$ & $0-1$ & 0.867019 \\
1y DT p0 & $97-137$ & $2-12$ & $0-1$ & 0.931036 \\
& 1y DT p1 & $90-129$ & $2-11$ & 0 & 0.9777077 \\
1y DT p2 & $89-128$ & $1-10$ & 0 & 0.977764 \\
6m DT p0 & $89-127$ & $1-10$ & 0 & 0.984398 \\
6m DT p1 & $87-125$ & $1-10$ & 0 & 0.991521 \\
1m DT p0 & $84-122$ & $0-8$ & 0 & 0.9992 \\
Quadrupoles & 1m DT p1 & $82-120$ & $0-6$ & 0.9998 & \\
\hline
\end{tabular}

Table 8.3: Reliability results for dipole and quadrupole quench heater power supplies after 20 years. HPS: Lost Power Supplies (needed spare), UM: Unprotected Magnets, LM: Lost Magnets, $R_{L M}$ probability of not loosing any magnet after 20 years of LHC operation.

monthly discharges should be done in order to ensure the performance (main failure probability below $1 \%$ ). The post-mortem information does not have such an important effect if discharge tests are carried out monthly. This is due to the low quench rate: while the post-mortem data obtained from a quench will only involve the quenched magnets, the discharge test provides information about the 2016 main magnets in the arcs.

\subsection{Sensitivity Analysis}

RESQP has been used to compute the sensitivity of the results to drifts on the component failure rate and/or the expected quench occurrence.

The same FAST frequencies than for the local quench detectors have been used for the power supplies. $\omega_{1}=11$ and $\omega_{2}=13$.

Figure 8.9 shows the sensitivity of the maximum number of lost dipoles with a $90 \%$ confidence level after 20 years of LHC operation with the maintenance policies $1 y$ DT $p 0$ (left) and $1 y D T p l$ (right). The same has been done for the quadrupoles in Fig. 8.10.

An important result observed is the effect of the quench rate. Although the number of failures clearly increases with higher quench rate when the post-mortem information is ignored (plots on the left), when this information is taken into account (plots on the right) its contribution to the output becomes much less important, above all in the case of the quadrupole magnets.

When the magnet is not unprotected, a natural quench acts like a discharge test. It has then 

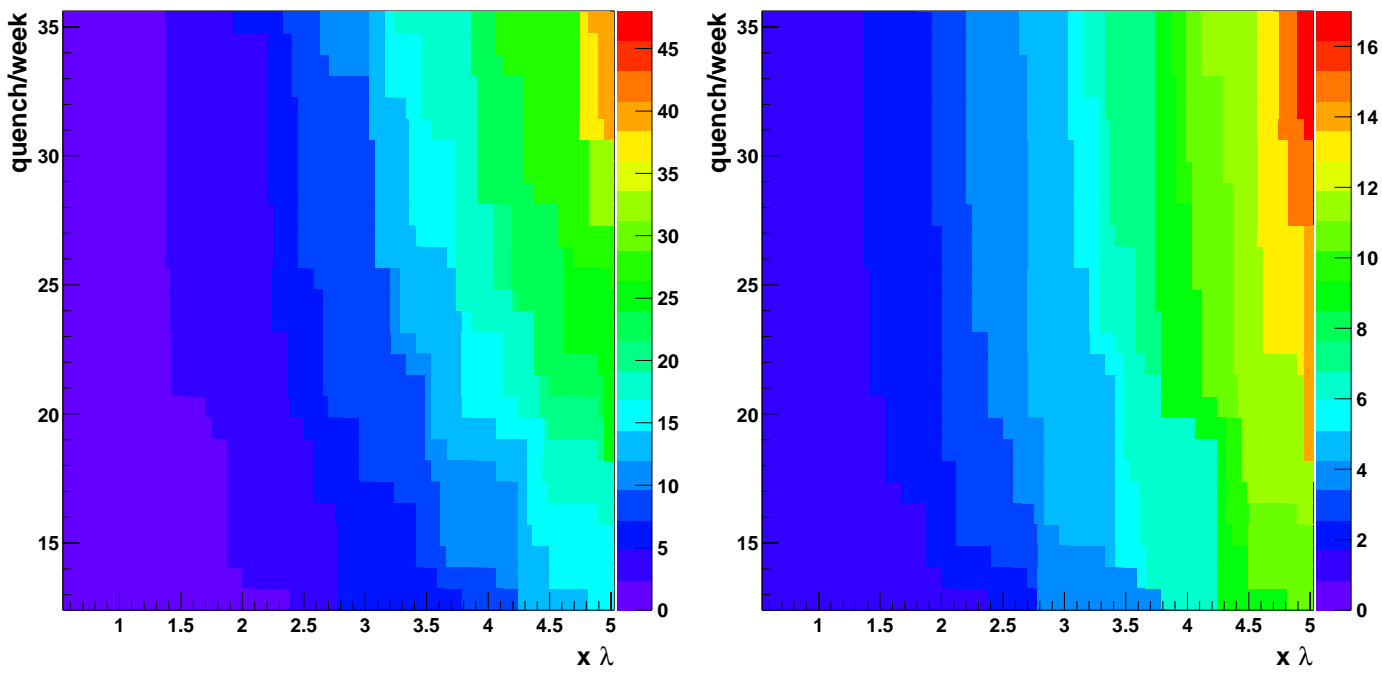

Figure 8.9: Sensitivity of maximum number of lost dipoles ( $90 \%$ confidence level) to component failure rate and quench rate with maintenance policies 1 y $D T p O$ (left) and 1 y $D T p 1$ (right).
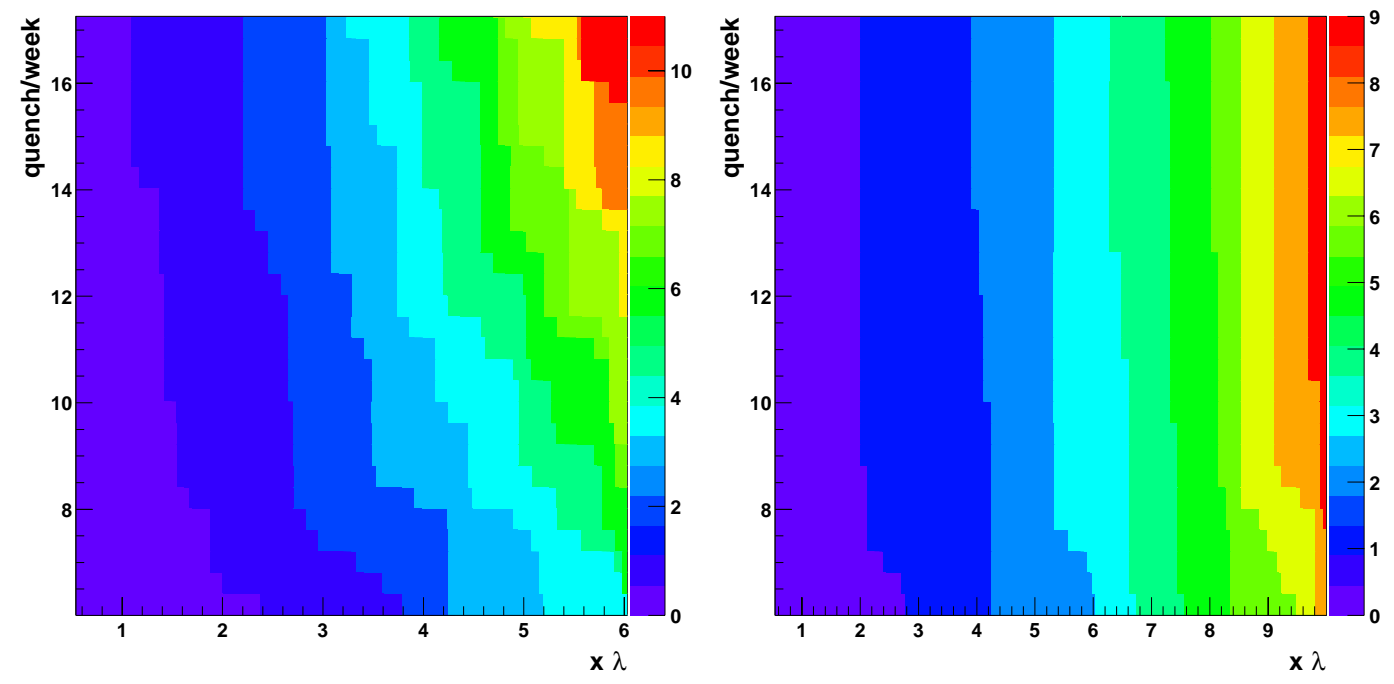

Figure 8.10: Sensitivity of maximum number of lost quadrupoles (90\% confidence level) to component failure rate and quench rate with maintenance policies 1 y $D T p O$ (left) and $1 y D T p l$ (right).

a benign effect on the protection system since the damaged power supplies will be detected and repaired. A higher quench rate will therefore increase the danger in the unprotected magnets but will improve the performance of the rest. The higher the system redundancy, the more important this positive effect will become. This can be clearly seen in the quadrupole magnets, whose level of redundancy (one out of two) is higher than in the dipoles (three out of four). 


\subsection{DQHDS Maintenance Conclusions}

This chapter has been focused on the maintenance optimisation and validation of the topology. The main conclusions are:

- Voltage monitoring is an input for the power permit but will not increase quantitatively the system reliability.

- Yearly discharge tests increase a $25 \%$ the reliability of every heater power supplies after 20 years.

- Post-mortem information is as important as yearly discharge tests. Its weight is lower if the discharge tests are performed monthly.

- There is not an important difference between the considered maintenance policies as soon as the post-mortem information is taken into account (policies 1 and 2).

- If yearly discharge tests are carried out, the probability of not suffering any main failure after 20 years is $77 \%$ for the dipoles and $98 \%$ for the quadrupoles. The probability of not suffering more than one failure is $97 \%$ and $99.9 \%$ respectively.

- In protection systems with high redundancy degree the initiator (e.g. quench, fire) can have a benign effect on the total availability if the post-mortem information is properly used. 


\section{Chapter 9}

\section{Reliability of the LHC Energy Extraction Systems}

\subsection{Energy Extraction Design and Operation}

The energy extraction is a part of the normal operating procedures in the LHC machine. Since energy recuperation is possible in some circuits (e.g. main magnets), the energy extraction will not be used for ordinary de-excitation of the magnet chains but only after several well defined events:

- Quench in a magnet coil, a superconducting busbar or a current lead.

- Risk of damage to other components in the power circuit (e.g. no water flow for a certain time in the $13 \mathrm{kA}$ water-cooled cables).

- Failure in the power converters or in mains which could trip the powering.

Energy extraction is required by some of the $13 \mathrm{kA}$ and $600 \mathrm{~A}$ circuits, hence two different extraction systems have been designed.

\subsubsection{3 kA Energy Extraction}

In Chapter 6 it was shown the necessity of a fast de-excitation of the circuits in the arcs after a quench in order to avoid overheating of the by-pass diodes.

The extraction facility could be implemented in the circuit in series or in parallel connection. In a series system the dump resistor is bridging the extraction switches, which are inserted in series with the load. In a parallel system the dump resistor is bridging the magnet chain and the opening switches are located at the terminals of the power converter.

The parallel topology allows operation without by-pass across the the power converter, however it cannot be divided into smaller subsystems which would enable a significant reduction of the maximum voltage to ground and the sharing of the total dissipated energy. Moreover, if the extraction is connected in parallel, the converter will raise to half the peak extraction voltage for the opening delay between subsystems, and during this time the first opening switch will be subjected to the full peak extraction voltage. In a double-series topology the voltage across the converter will never arise above a quarter of the peak voltage. 

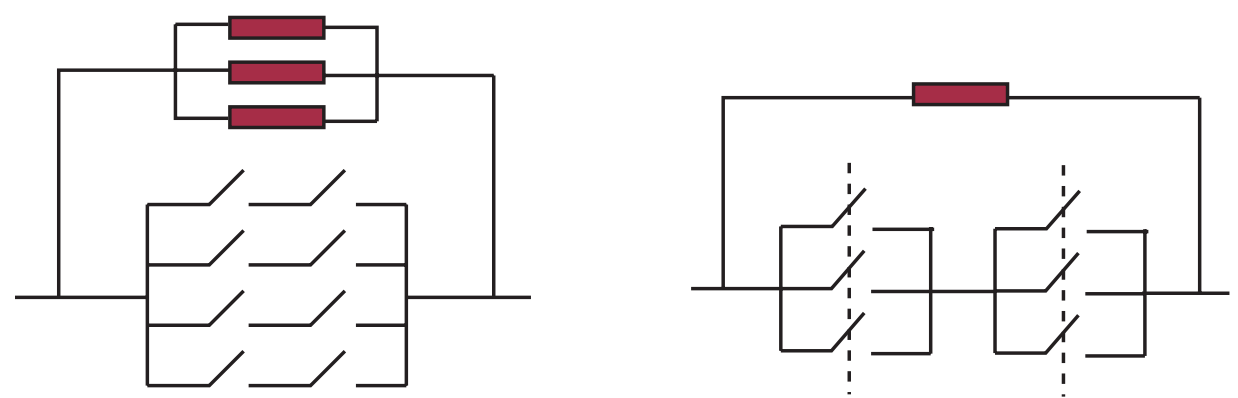

Figure 9.1: Switches and extraction resistors topologies for $13 \mathrm{kA}$ (left) and $600 \mathrm{~A}$ (right) LHC circuits.

Hence, a double series topology has been chosen for the dipole and quadrupole arc circuits (see Fig. 9.1). The dipole circuit has two symmetrically placed $660 \mathrm{MJ}$ subsystems, one in the even point, at the negative pole of the converter, and one in the odd point, at the electrical circuit midpoint (see Fig. 3.8). The circuit earthing in the electrical midpoint of one of the two dump resistors divides by four the maximum voltage to ground $(490 \mathrm{~V}$ instead of $1960 \mathrm{~V})$, and reduces the needed voltage rating of the switches.

A parallel topology has been also chosen for the arc quadrupole circuits, which require only one energy extraction system per circuit.

The technology of the switches had to be chosen between semiconductor based devices like thyristors or GTO with anti-parallel diodes, and electro-mechanical DC breakers. Both technologies have been widely used at CERN during the last years mainly in LHC test benches. The selection must be based on reliability and radiation hardness. DC breakers have been chosen due to several advantages:

- Eletro-mechanical breakers can be equipped with two totally independent release systems, one passive (de-energizing of a holding coil) and one active (pulsed release), whereas the semiconductor devices entirely rely on a single active system.

- The voltage drop across a mechanical breaker is typically $60 \mathrm{mV}$ at $4 \mathrm{kA}$, while it is $1.4 \mathrm{~V}$ for a conducting power thyristor or a GTO. Hence, a complete array of mechanical breakers for $13 \mathrm{kA}$ would generate power losses up to eight times lower than an equivalent thyristor switch array.

- A DC breaker is significantly more radiation tolerant than a semiconductor device.

- While the semiconductor switch needs water-cooling, the mechanical breaker is natural aircooled. Therefore, only the DC switch-gear solution can be maintained closed in case of a failure in the mains, which would stop the LHC cooling pumps.

- The breakers are by nature a protection device and they are mainly used to protect highly valuable installations against short-circuits. This implies more reliable components.

The application of electro-mechanical switches as energy extractor requires:

- Very high long term reliability.

- No mechanical catch and latch system, the closed state must be maintained only by excitation of the holding coil. 
- Two completely independent release systems in order to improve availability.

- Magnetic displacement of the arc for fast and reproducible arc extinction.

- High overload and breaking capability.

- Low voltage drop and losses.

- Protection by overload detector.

- Design based on industrial components, which have been in production for at least ten years.

These demands are not satisfied by any breaker existing on the market. The design and manufacture of a suitable breaker have been carried out together with the Russian industry (UETM in Ekaterimburg). The result is a fast breaker, with typical total opening times of $5 \mathrm{~ms} \pm 1 \mathrm{~ms}$ for the pulsed release and $20 \mathrm{~ms} \pm 2 \mathrm{~ms}$ for the under-voltage release. The design comprises components from three existing DC breakers presently protecting sub-stations, locomotives and steel works in former USSR Republics, China and India.

\subsubsection{A Energy Extraction}

In Table 7.13 were listed the energy extraction requisites for the different correction circuits. Due to the low ramping voltage and the high dump resistance, the $600 \mathrm{~A}$ extraction facilities accept both series and parallel topology. However, for external individual extraction system a series topology is preferred, as the converter already has a crowbar by-pass. For the converter-integrated facilities, which will not be studied here, a parallel system is preferable.

Two different $600 \mathrm{~A}$ energy extractors are currently under evaluation. They were also developed by the Russian industry (BINP in Novosibisk).

The short opening time is a big issue for this system. In both proposals the extraction is based on two, series-connected, fast mechanical AC switches using standard 3-pole electro-mechanical AC breakers, retro-fitted with DC arc chutes and capacitive snubber circuits for arc suppression. In order to maximise availability, the military version is used.

The current will be shared by the three poles in parallel, which are simultaneously operated by a common mechanical axis (see Fig. 9.1). Like for the $4.5 \mathrm{kA} \mathrm{DC}$ breaker, also these AC switches have two independent modes of release.

\subsubsection{Operation and Reliability}

The explanation of the different energy extraction designs has been focused on the breakers since, as the only active part, they are the most important regarding the reliability issues. Consequently the dangerous scenarios and possible failures will be due to malfunctioning of these components.

During powering of the associated magnet chain, the energy extraction facility shall always be operational and ready for extraction independently of the state of any infrastructure. The most important operational solutions in order to reach such a taught demand are:

- The DC breakers are opening switches. Repetitive closing with current must be avoided in order to avoid damage to the breaker contacts.

- All logic is made in hard-wired electronics, hence no vital safety features rely on software. 
- The local powering and controls are fed from the UPS.

- The facility is a part of the power enabling chain (i.e. Power Permit) as well as of the Power Abort Link.

- During the closing process (e.g. before Power Permit after a quench), all the switches must close and remain closed for at least $5 \mathrm{~s}$. For the $13 \mathrm{kA}$ circuits, the breaker rating allows continued operation with only three branches. During a machine run one branch may fall out (opening by accident) without compromising the operation. In the case that more than one branches are opened all the other breakers of the facility will be forced to open.

- The two release mechanisms of each breaker are always triggered simultaneously. The correct operation of both procedures is monitored in order to improve reliability.

- For the dipole circuits, in case of an accidental opening of one of the two facilities in the same sector circuit, the second facility of the same sector must be triggered for opening (through the power abort link). Otherwise, the open facility would have to absorb up to 1.3 GJ and a number of resistor sensors risk destruction.

The main failure of the energy extraction is the unlikely event called switch-opening failure, which will occur if one branch of a system remains closed after an opening command (in the case of the $600 \mathrm{~A}$, both series-connected switches must remain closed). This is one of the most dangerous machine malfunctionings since several cold diodes in the main magnets and corrector circuits could be destroyed if the stored energy is not properly released. Two different solutions have been considered.

The original strategy was to generate the needed resistance into the quenching circuit by firing the quench heaters of several magnets. This is a safe solution since the failure probability will vanish but, on the other hand, it will lead to a huge load for the cryogenic system and dangerous mechanical and electrical stresses in the magnetic elements.

The second solution, recently considered, is to connect in series to the facility an ultimate current breaking component. This includes an externally triggered fuse, which is being developed for the fusion project ITER (International Thermonuclear Experimental Reactor) by NIIEFA in St Petersburg. The feasibility and utility of each solution are analysed below and selecting the preferable option is the main goal of this chapter.

\subsubsection{High Current Controllable Fuses}

The $13 \mathrm{kA}$ and $600 \mathrm{~A}$ fuses are current commutation devices originally developed by D.V. Efremov [78]. They consist on a main current carrying copper element, which carries the current in the circuit. It has a small ring-shaped cross-section. When required, a controlled explosion in a capsule with gassing composition and an igniter, pushes a cylindrical piston made of insulation material through the busbar and interrupts the current.

The operation of the fuse is initiated by a controlled impulse from an external triggering device. A capacitor is discharged into a thin filament (igniter) fixed to the gassing composition. The pressure generated by the gas is around $30 \mathrm{MPa}$. The arc created due to the rupture of the busbar is rapidly extinguished by the obstruction of the gaps provided by the cylindrical piston.

The main current conductor, the piston and the capsule composition have to be replaced after every operation. The other components are designed for at least 10 usages. 


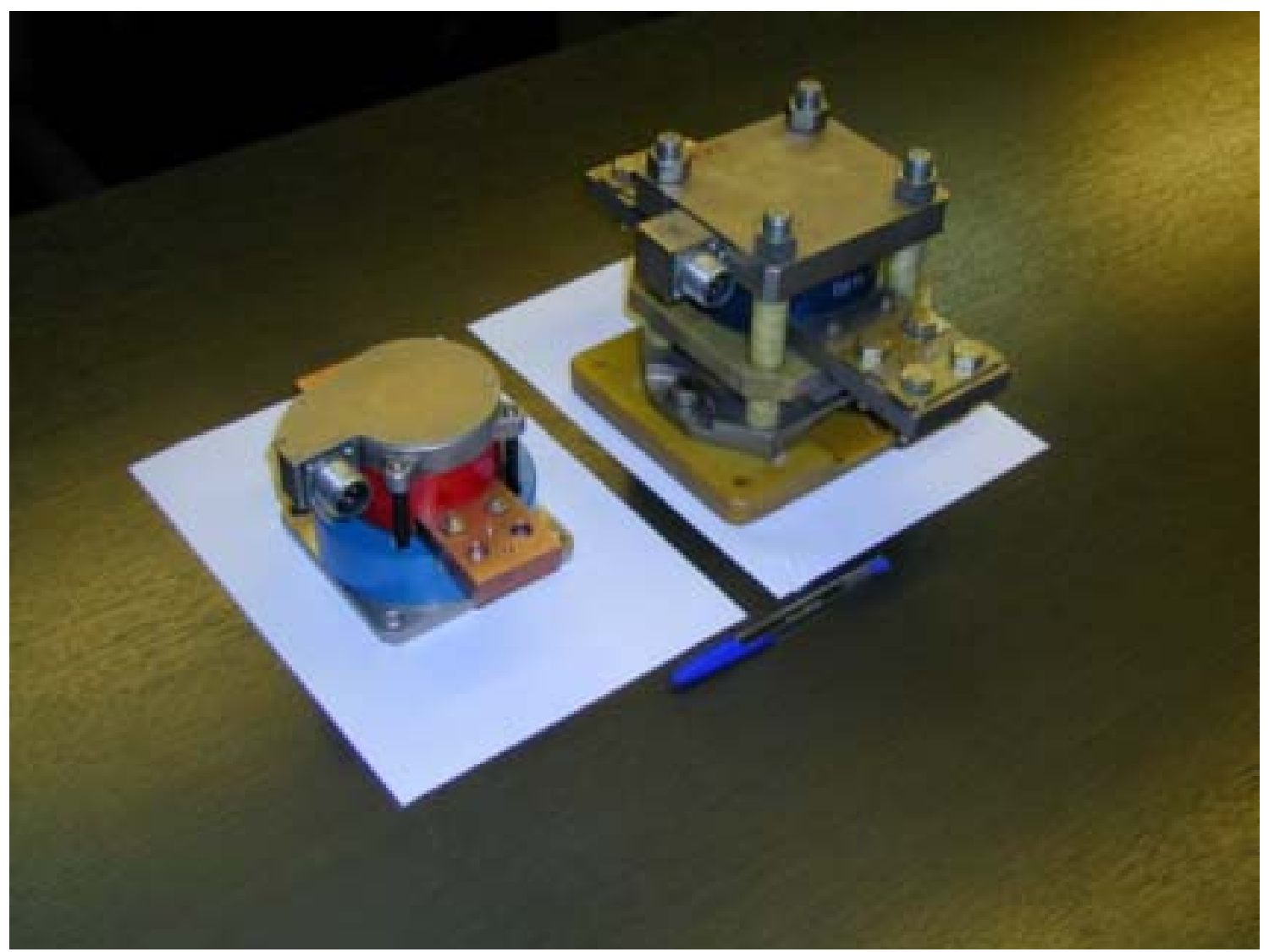

Figure 9.2: $600 \mathrm{~A}$ and $13 \mathrm{kA}$ controllable fuses for the LHC energy extraction facilities..

Both, the $13 \mathrm{kA}$ and $600 \mathrm{~A}$ fuses are still on the prototype phase. This can be an important drawback in order to be used in the LHC energy extraction system. Nevertheless, some tests have been carried out [78] and, although the experience is still rather low for stating confident conclusions about their reliability, the feasibility of this solution and the worthiness of the investment must be analysed.

\subsection{Reliability Model}

The multi-state model used by RESQP is fully suitable for analysing the reliability energy extraction facilities explained above. However, the failure distributions described in Chapter 4 are only applicable on continuous time events.

In devices like the electro-mechanical breakers, clock time is not the best scale to describe lifetime, and the reliability data is usually expressed by the manufacturer as the failure probability on demand. Lifetime is then redefined as the number of demands successfully completed prior to a failure. The usual reliability theory in continuous time that has been used along this thesis cannot be applied and must be adapted to discrete time. The most common statistical parameters in continuous time have been redefined in [79, 80, 81].

Being $K$ a random variable defined on the set of positive integers $N^{*}$ called the discrete lifetime, the probability mass function $f$ gives the probability of the device to fail on any demand: 


$$
f(k)=P(K=k)
$$

The reliability function in discrete time $R(k)$ gives the probability of the device not to have failed before the next demand to $k$ :

$$
R(k)=f(K>k)=1-\sum_{i=1}^{k} f(i)
$$

The failure rate in discrete time $\lambda$ gives the conditional probability of failure on demand $k$ given that it did not fail before:

$$
\lambda(k)=P(K=k \mid K \geq k)=\frac{f(k)}{R(k-1)}
$$

It is important to notice that $\lambda$ is a probability, hence it is bounded by 1 , contrary to the failure rate in continuous time defined in Eq. 4.2. Finally the Mean Time to Failure is defined as

$$
M T T F=E(K)=\sum_{k=1}^{\infty} k f(k)=\sum_{k=0}^{\infty} R(k)
$$

The exponential failure rate distribution has been used until now for describing the behaviour of a device during the second phase of the bath-tube curve (i.e. random failure phase). The equivalent in discrete time is the geometric distribution, which as for the exponential is characterised by a constant failure rate:

$$
f(k)=p(1-p)^{k-1}
$$

thus $\lambda(k)=p$ with $p$ being the probability of failure on any demand. RESQP model explained in Chapter 5 can be used for devices with memoryless behaviour if Eq. 5.6 is replaced by its analogue in discrete time:

$$
p_{i}(k)=\sum_{j=1} k(1-p)^{i-1} p
$$

Instead of time $t$, the number of demands will be used for the computations, hence input parameters such as maintenance frequency or machine lifetime must be defined according to the expected quench rate.

The big advantage of facing the problem in discrete time is that the distinction between reliability and availability that required the use of random walk theory to compute the main failure probability, becomes meaningless when using reliability on demand. In discrete time, the dangerous failure probability given by RESQP will be equal to the main failure probability.

Ageing and wear out effects can be also modelled with RESQP using discrete failure functions analogue to the continuous Weibull distribution. These cases will not be treated here. A detailed overview of these reliability models can be found in [82]. 


\subsection{Reliability of the $13 \mathrm{kA}$ Energy Extraction Systems}

Like for the quench heater power supplies studied in the previous chapter, the basic topology and design of the energy extraction facility was already defined when this study started. Therefore the reliability analysis is focused on the maintenance policies in order to optimise the performance and, above all, on selecting the best action to be taken after a switch open failure.

\subsubsection{Failure Modes and Maintainability}

Since discrete time modelling is used, no distinction must be done between dangerous and main failures. The possible malfunctionings will be then:

- Main Failure: Not enough switches are opened on demand and the ultimate current breaking fuse is not triggered (if the fuse solution is adopted).

- Spurious Energy Extraction: At least two branches are opened with current in the magnets. Beam must be unnecessarily dumped..

This last failure is analogue to the false quench of the quench detectors. If one branch is opened during machine operation no action will be taken until next Power Abort since, like in the quench detectors, the worst consequence would be an unscheduled beam dump. Since the power permit cannot be given unless all the energy extraction switches in the machine are closed, the failed breaker must be compulsory repaired or replaced after the next Power Abort of the subsector. This constraint fixes the maintainability against spurious extraction.

The maintenance against switch opening failures (switches not opened on demand) is more flexible than against the spurious opening. The reliability study considers inspection and repairs carried out yearly, monthly, or after each demand on the specific facility.

\subsubsection{Input Parameters}

\section{Expected Quench Rate}

Like for the other systems analysed, the quench rate is a rather unpredictable parameter and the confident of the study results will rely on their sensitivity to this input. Nevertheless a nominal value can be set from the LHC design baselines in order to have a starting point for the analysis.

As for the quench detectors, this value will be the one used by the hardware designers as maximum number of quenches that the weakest parts of the system can afford: 24 quenches per operational week. This value includes the induced quenches from magnet to magnet. Here we should only consider the number of switch aperture demands.

Experience in String 2 shows that, in average, a quench in a main magnet propagates to its two neighbours, hence the number of power aborts in the main circuits will be a third of the total quenched magnets (not considering possible propagations to the correctors).

This means 24 quenches per week from dipoles and quadrupoles indistinctly will correspond to 8 natural quenches per operational week. Since the quench can propagate from the dipole to the quadrupole and vice-versa, the energy will be extracted from all the main magnets in the cryostat, thus four extraction facilities will be opened. This means a total of 960 apertures per year, thus 30 yearly requests per facility (32 energy extraction banks). Under this hypothesis the number of yearly apertures per facility can be computed as 1.25 times the expected number of quenches per week in dipoles and quadrupoles (considering quench propagation). 


\section{Reliability Data}

The failure rate of the switch breakers varies depending on the source. It is planned to use similar circuit breakers for the ITER project. The values managed by the researchers of its energy extraction system are 0.002 , under conservative assumptions, and below 0.0002 , with optimistic hypothesis.

In any case, these values are obtained from few experimental data and the novelty research of the LHC switches makes impossible to define at the present state which will be their number of failures on demand. Sensitivity analysis will be again essential. The assumed failure rate will be from $10^{-2}$ to $10^{-4}$ on demand. Tests carried out by CERN have given a failure probability on demand of the $13 \mathrm{kA}$ breakers better than $10^{-4}$ [83].

It is even more difficult to estimate de failure probability for the pyro-breakers. These fuses are still under development and since they can only be used once, the reliability tests are rather expensive. Very few experimental data have been obtained [78], hence only theoretical assumptions will be used. For the ITER project it has been estimated a maximum failure rate in the range of $10^{-4}-5 \cdot 10^{-5}$. Tests carried out by the manufacturer do not show such a promising performance. Therefore, much more pessimistic values are used in the next calculations: $10^{-2}-10^{-4}$ on demand.

Although the reliability data of the fuses are provided by the manufacturers and ITER researchers in terms of availability on demand, these are unrepairable devices, which makes too optimistic the hypothesis of constant failure rate on discrete time. Since the fuses cannot be tested during operation, some of them will be requested to open several years after others were. It is reasonable to assume that the probability of a fuse to fail if it is requested after ten years of LHC operation can be rather higher than the failure probability of a fuse triggered during the first year. The fuse will follow an exponential distribution in continuous time. The failure rate has been estimated from the available data. It has been assumed that the probability of failure is constant between switch opening requests (i.e. between natural quenches in the subsector), hence, the failure rate of the fuses will depend on the quench rate applied. Table 9.1 shows the conversion from the given data to the used values.

\begin{tabular}{ccc} 
Quench Rate & $\lambda_{\text {fuse }}=10^{-2}$ & $\lambda_{\text {fuse }}=10^{-4}$ \\
\hline $4 \frac{\text { quench }}{\text { week }}$ & $58479 \mathrm{~h}$ & $5839707 \mathrm{~h}$ \\
$8 \frac{\text { quench }}{\text { week }}$ & $29239 \mathrm{~h}$ & $2919854 \mathrm{~h}$ \\
\hline
\end{tabular}

Table 9.1: Mean Time to Failure (MTTF) of the fuses used in the continuous model.

\subsubsection{Reliability Results}

The goal of this chapter is not to give absolute values about the reliability but to study the influence of the highly uncertain input parameters and the impact of including the explosive fuse on the design. Hence, FAST method (see Chapter 5) included in RESQP together with the model modifications for discrete time explained above have been used.

Figure 9.3 shows the main failure probability after 20 years for the $13 \mathrm{kA}$ energy extraction facilities if only electro-mechanical breakers are implemented. The result is computed in function of the failure rate on demand for a single switch and the expected number of natural quenches per week. The analysis has been done with two different maintenance policies:

- Yearly checks: repairs are carried out only during the winter shutdown of the machine. 
- Repair after demand: post-mortem data is used to detect failures of the actioned facility. Every opening demand is analysed a posteriori in order to detect malfunctioning components and repair is carried out before next power permit.
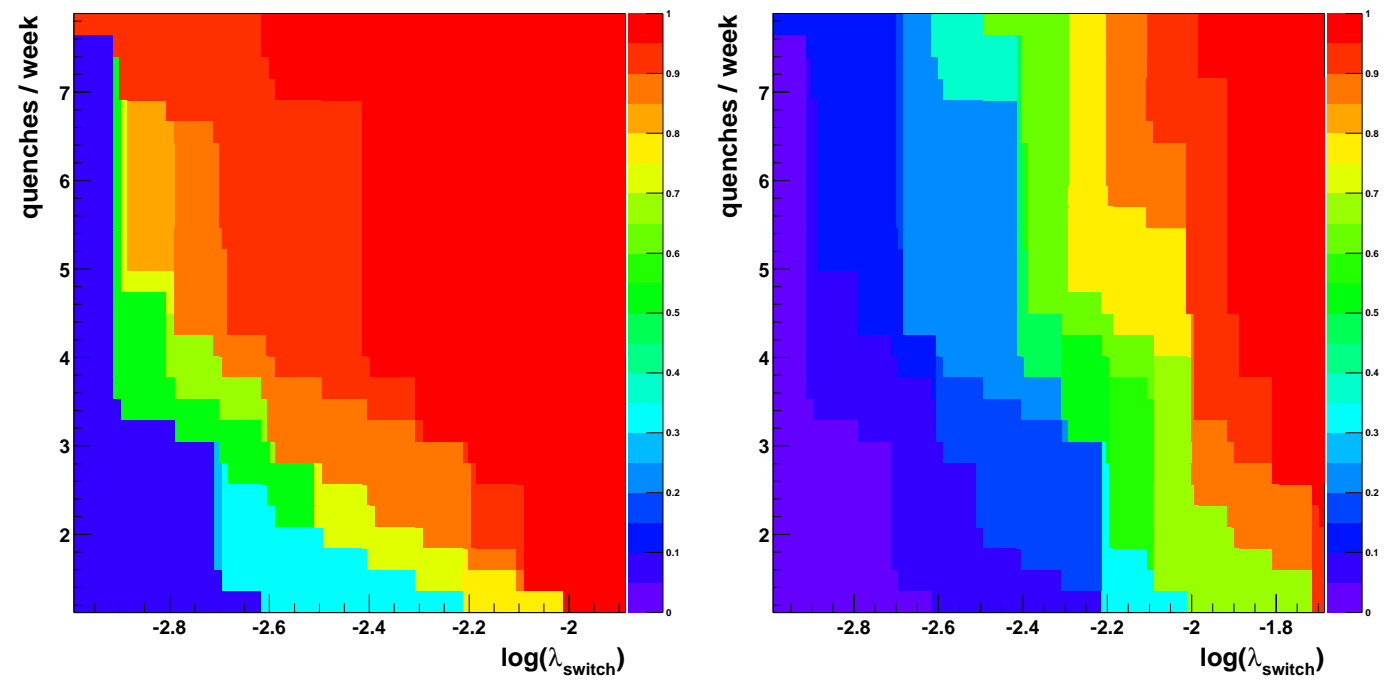

Figure 9.3: Failure probability of the $13 \mathrm{kA}$ energy extraction facility after 20 years with yearly repairs (left) and repairs after each demand (right).

It can be seen in Fig. 9.3 that the probability of failure gets too high when repairs are made only during the long shutdowns of the machine. The most convenient will be to repair the switches after the failure has been detected by the post-mortem information. In that case, for normal quench rates (i.e. no more than two natural quenches per week) the failure probability remains below $5 \%$ if the failure rate of the breakers stays below 0.005 .

In the previous chapter, it was seen that the reliability of a highly redundant system becomes almost independent from the initiator rate when post-mortem data is taken into account. In the case of the $13 \mathrm{kA}$ energy extraction the redundancy level is not high enough to show this effect.

In order to evaluate the impact of a controlled fuse connected in series to the breakers, the failure probability has been computed for different quench rates ( 4 and 8 natural quenches per operational week). In this case, FAST analysis has been applied for the two reliability parameters: breaker and fuse failure rates. The maintenance policies are the same than above (see Fig. 9.4). The results for some significative input values are listed in Tables 9.2 and 9.3.

A very useful parameter for evaluating the worthiness of implementing a safety device into a system is the Safety Integrity Level (SIL). The SIL of a protection system is an integer value from 1 to 4, which is computed from its reliability and/or average availability on demand. A minimum required SIL can be defined for any safety system depending on the likelihood of the initiator (e.g. quench) and the consequences of an incorrect performance of the protection system.

According to IEC-61508 recommendations [53], the consequences of a main failure in the LHC energy extraction after a request can be defined as Severe (downtime due to full arc quench between 3 and 20 days and/or around $10^{5}-10^{6} \mathrm{CHF}$ lost, see Appendix C) and the event likelihood as Frequent (more than one event per year, see Appendix C). The international standards recommend a SIL 3 level protection for such a system. 

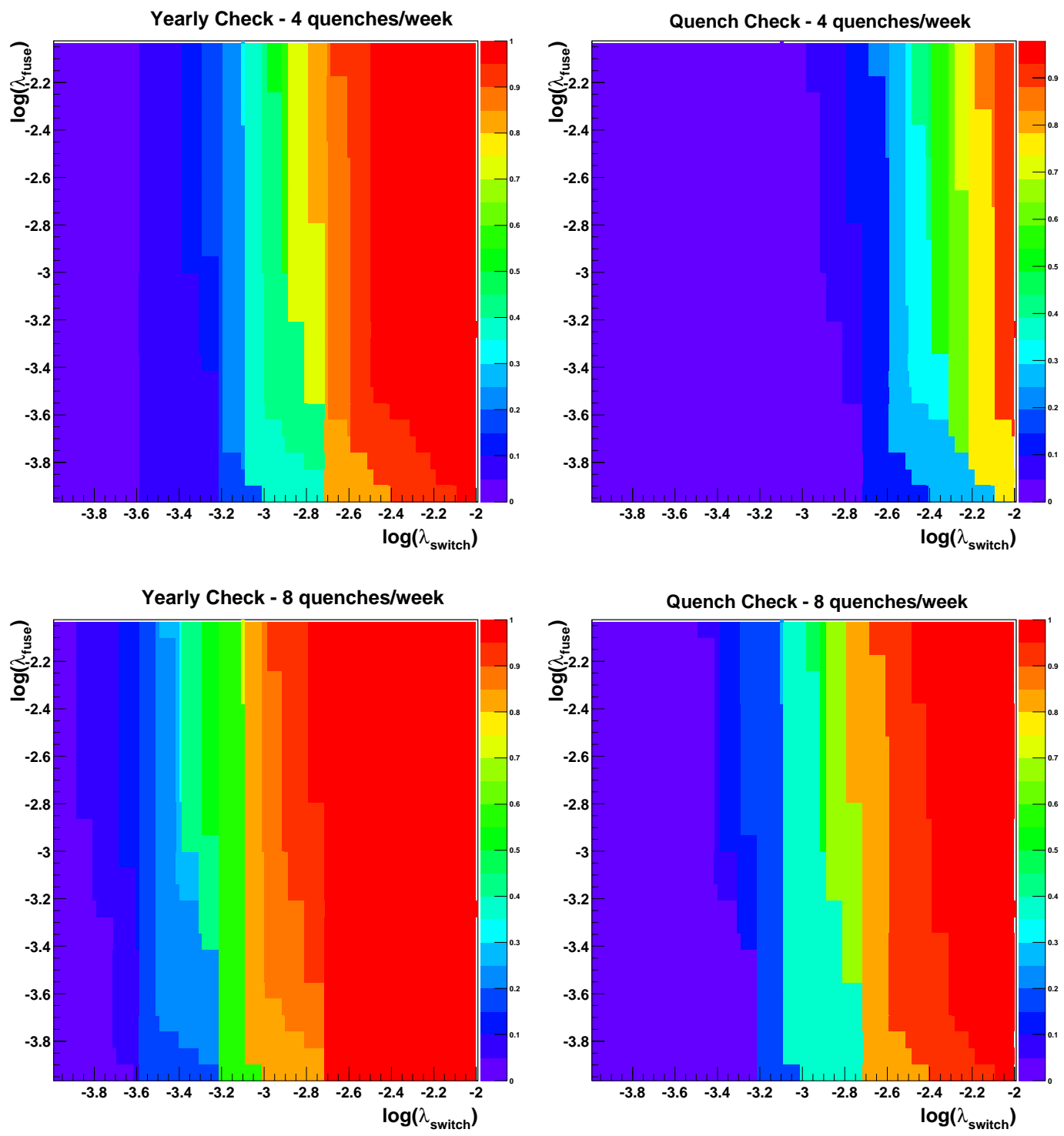

Figure 9.4: Failure probability during 20 years of the $13 \mathrm{kA}$ energy extraction facility with an implemented controlled fuse.

\begin{tabular}{r|ccc|ccc|}
\multicolumn{2}{c}{$4 \frac{\text { quench }}{\text { week }} \equiv 15 \frac{\text { demand }}{\text { year.facility }}$} & \multicolumn{2}{c}{$8 \frac{\text { quench }}{\text { week }} \equiv 30 \frac{\text { demand }}{\text { year.facility }}$} \\
\cline { 2 - 7 } & $\lambda_{s}=10^{-2}$ & $\lambda_{s}=10^{-3}$ & $\lambda_{s}=10^{-4}$ & $\lambda_{s}=10^{-2}$ & $\lambda_{s}=10^{-3}$ & $\lambda_{\text {switch }}=10^{-4}$ \\
\cline { 2 - 7 } No Fuse & $100 \%$ & $43.3 \%$ & $0.57 \%$ & $100 \%$ & $89.3 \%$ & $2.2 \%$ \\
$\lambda_{\text {fuse }}=0.01$ & $100 \%$ & $41.6 \%$ & $0.54 \%$ & $100 \%$ & $89.2 \%$ & $0.07 \%$ \\
$\lambda_{\text {fuse }}=0.001$ & $99.9 \%$ & $13.5 \%$ & $0.15 \%$ & $99.9 \%$ & $62.8 \%$ & $1.02 \%$ \\
$\lambda_{\text {fuse }}=0.0001$ & $53.2 \%$ & $1.6 \%$ & $0.02 \%$ & $84 \%$ & $11.8 \%$ & $0.13 \%$ \\
\cline { 2 - 7 }
\end{tabular}

Table 9.2: Failure probability after 20 years of the $13 \mathrm{kA}$ energy extraction with yearly maintenance $\left(\lambda_{s} \equiv\right.$ $\left.\lambda_{\text {switch }}\right)$.

RESQP has been used to compute the SIL in function of the switch failure probability and the expected quench rate ${ }^{1}$. Since the energy extraction is in a high demand mode of operation, the 


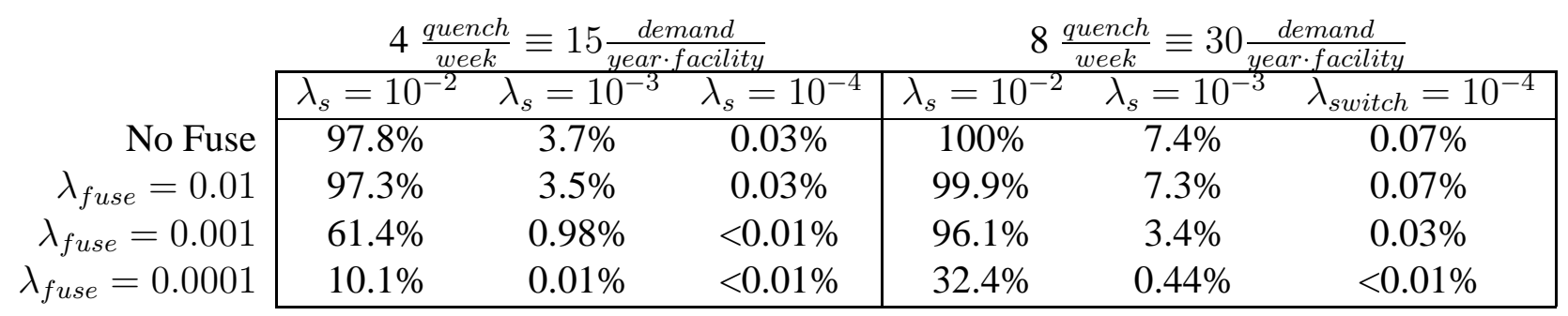

Table 9.3: Failure probability after 20 years of the $13 \mathrm{kA}$ energy extraction with maintenance after opening demand $\left(\lambda_{s} \equiv \lambda_{\text {switch }}\right)$.

probability of a dangerous failure (i.e. facility unavailable) per hour is used to determine the SIL value (see Appendix C).

Figure 9.5 shows the safety integrity levels of the $13 \mathrm{kA}$ energy extraction systems without fuse in function of the weekly opening demands per facility and the switch failure rate. It can be seen that SIL 3 can be easily accomplished if repairs are carried out after demand.
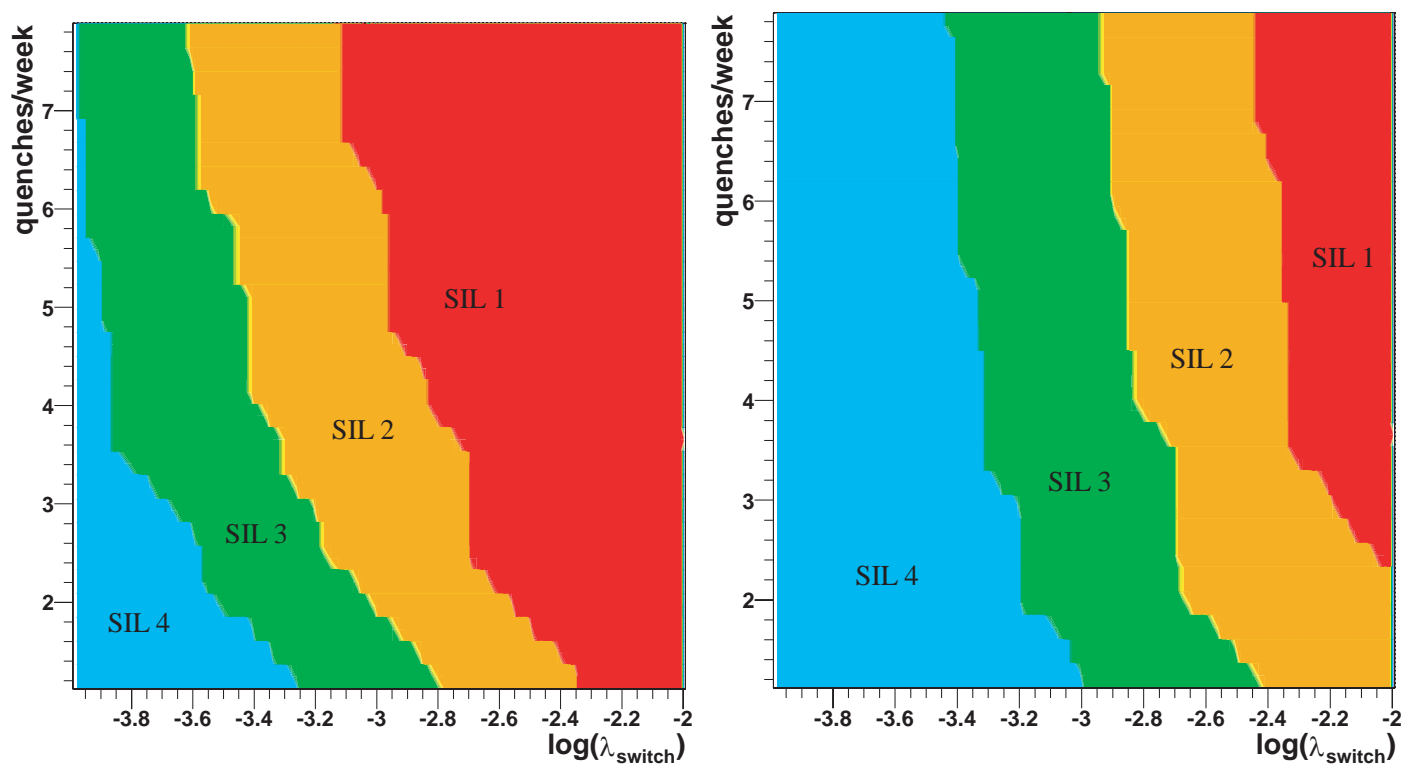

Figure 9.5: Safety Integrity Level of the $13 \mathrm{kA}$ energy extraction facility without fuse for yearly repairs (left) and repairs after each demand (right).

\subsection{Reliability of the 600 A Energy Extraction Systems}

A total of 176 corrector circuits are protected by the same number of $600 \mathrm{~A}$ energy extraction facilities. The failure modes are the same than for the main circuits energy extractors. The study has been focused on the influence of the expected quench rate and reliability data on the switches availability and the utility of including a specially designed fuse on the design.

\footnotetext{
${ }^{1}$ Actually the initiator occurrence should not affect on the system SIL since it only depends on the system's state. However, in our case the quench rate affects on the system maintenance frequency.
} 


\begin{tabular}{lccc} 
& EE Facilities & $\begin{array}{c}\text { Quenches per } \\
\text { Detector }(20 \mathrm{y})\end{array}$ & $\begin{array}{c}\text { Total } \\
\text { Quenches }(20 \mathrm{y})\end{array}$ \\
\hline MCS & 16 & 20 & 320 \\
MCD & 16 & 10 & 160 \\
MO & 16 & 1.0389 & 16.62 \\
MO & 16 & 1.6883 & 27.01 \\
MS & 32 & 1.2987 & 41.55 \\
MS & 32 & 1.5584 & 49.87 \\
MQT & 32 & 1.039 & 33.25 \\
MQTL & 8 & 0.7792 & 6.23 \\
MQTL & 8 & 0.2895 & 2.08 \\
\hline Total & $\mathbf{1 7 6}$ & $\mathbf{3 . 7 3}$ & $\mathbf{6 5 6 . 6}$ \\
\hline
\end{tabular}

Table 9.4: 600 A energy extraction facilities.

\subsubsection{Input Parameters}

\section{Expected Quench Rate}

The quenches in the corrector magnets are not detected locally but by global detectors that monitor the state of the whole circuit (see Chapter 7). Hence, in principle, the number of apertures should be the same as the number of quenches seen by the detector. Nevertheless, this is not true since the $600 \mathrm{~A}$ energy extraction facilities are also requested to open when one main magnet in the subsector quenches.

The expected number of opening requests due to quenches on the corrector circuits has been computed from the quench data of the corrector circuits detailed in Section 7.3. The values are listed in Table 9.4.

The low quench rate expected for the correction circuit gives an important weight on the model to the opening demands due to main magnet quenches. It is a reasonable approximation to consider the expected opening requests for the $600 \mathrm{~A}$ facilities as the same than it was for the $13 \mathrm{kA}$ ones.

\section{Reliability Data}

The problems we found for the $13 \mathrm{kA}$ switches are the same than for the $600 \mathrm{~A}$. There are not enough experimental data, and hypothesis can only be done from theoretical calculations. Therefore, the same reliability values used above will be applied to the energy extraction facilities for the corrector circuits. However, the experiments carried out by the manufacturers show a reliability performance slightly worse than for the $13 \mathrm{kA}$ breakers, whose design is based on a better known technology. The $600 \mathrm{~A}$ breakers failure probability is around $10^{-3}$ [83].

\subsubsection{Reliability Results}

Like it was done for the $13 \mathrm{kA}$ energy extraction systems, the probability of suffering a main failure after 20 years of the LHC operation has been computed for the 600 A facilities.

Figure 9.6 shows the failure probability of the systems without the controlled fuses. It can be seen that for this case, even when repairs are carried out after the opening request, the risk of 
suffering a main failure is very high. It can reach a 50\% probability for failure rate values not too pessimistic.
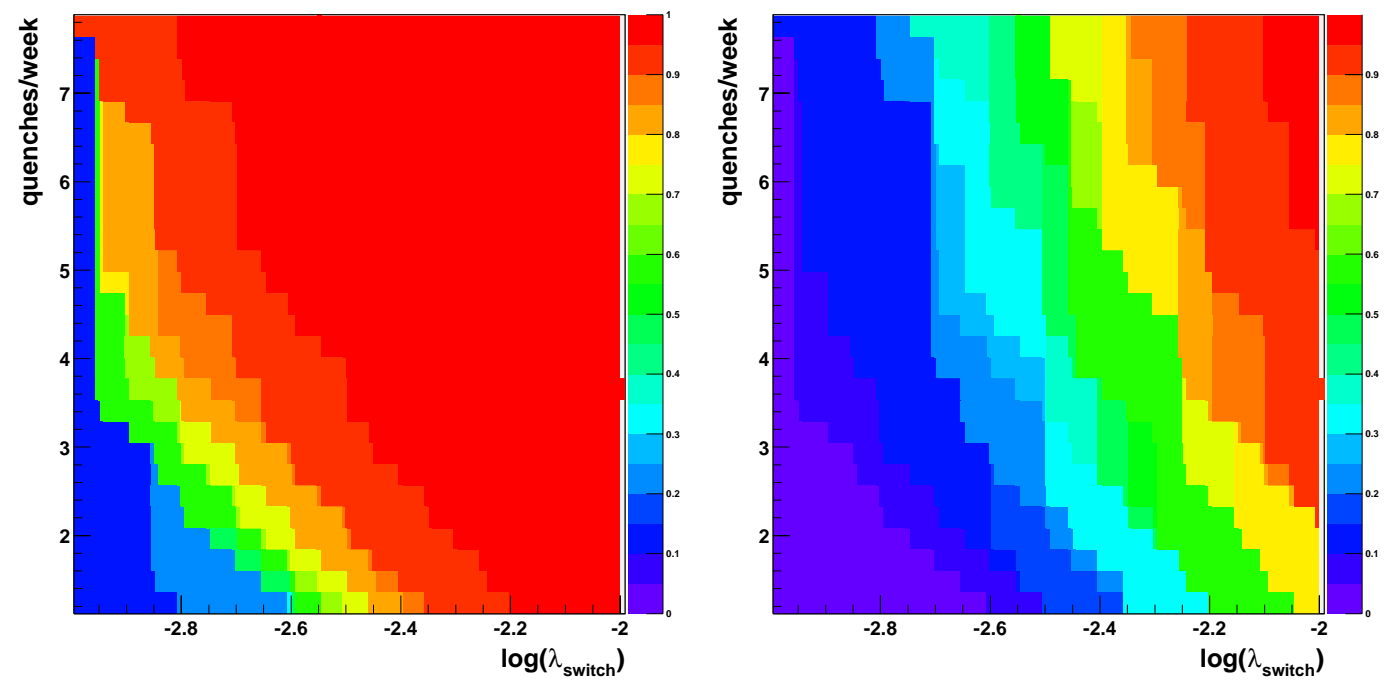

Figure 9.6: Failure probability of the $600 \mathrm{~A}$ energy extraction facility after 20 years with yearly repairs (left) and repairs after each demand (right).

For fuse performances better than $\lambda_{\text {fuse }}=0.001$ the failure probability still stays rather high (see Table 9.6). The pyro-fuse will not increase suficiently the relaibility of the facility (mainly because of its unrepairability nature), hence another solution has been considered. The space constrains for the $600 \mathrm{~A}$ energy extraction are not as taugh as they were in for the main magnets. It is feasible to install a third switch in series to the other two. The advantage will be the possibility of performing regular checks and a much lower cost. The main disadvantage is the use of a back-up device with the same technology, which can be dangerous in case of common source of failures.

The main failure probability for the energy extraction in corrector circuits after 20 years of LHC operation for some significative input values are listed in Tables 9.5 and 9.6.

\begin{tabular}{r|ccc|ccc|} 
& \multicolumn{2}{c}{$4 \frac{\text { quench }}{\text { week }} \equiv 15 \frac{\text { demand }}{\text { year.facility }}$} & \multicolumn{2}{c}{$8 \frac{\text { quench }}{\text { week }} \equiv 30 \frac{\text { demand }}{\text { year.facility }}$} \\
\cline { 2 - 7 } & $\lambda_{s}=10^{-2}$ & $\lambda_{s}=10^{-3}$ & $\lambda_{s}=10^{-4}$ & $\lambda_{s}=10^{-2}$ & $\lambda_{s}=10^{-3}$ & $\lambda_{\text {switch }}=10^{-4}$ \\
\cline { 2 - 7 } No Fuse & $100 \%$ & $58.8 \%$ & $0.89 \%$ & $100 \%$ & $96.9 \%$ & $3.5 \%$ \\
$\lambda_{\text {fuse }}=0.01$ & $100 \%$ & $56.9 \%$ & $0.85 \%$ & $100 \%$ & $96.3 \%$ & $3.35 \%$ \\
$\lambda_{\text {fuse }}=0.001$ & $100 \%$ & $20.4 \%$ & $0.23 \%$ & $100 \%$ & $59.3 \%$ & $0.92 \%$ \\
$\lambda_{\text {fuse }}=0.0001$ & $99.9 \%$ & $2.5 \%$ & $0.03 \%$ & $98.8 \%$ & $9.7 \%$ & $0.1 \%$ \\
Third Switch & $99.9 \%$ & $1.3 \%$ & $<0.01 \%$ & $100 \%$ & $9.8 \%$ & $<0.01 \%$ \\
\cline { 2 - 7 }
\end{tabular}

Table 9.5: Failure probability after 20 years of the 600 A energy extraction with yearly maintenance $(\lambda s \equiv$ $\lambda_{\text {switch }}$. 


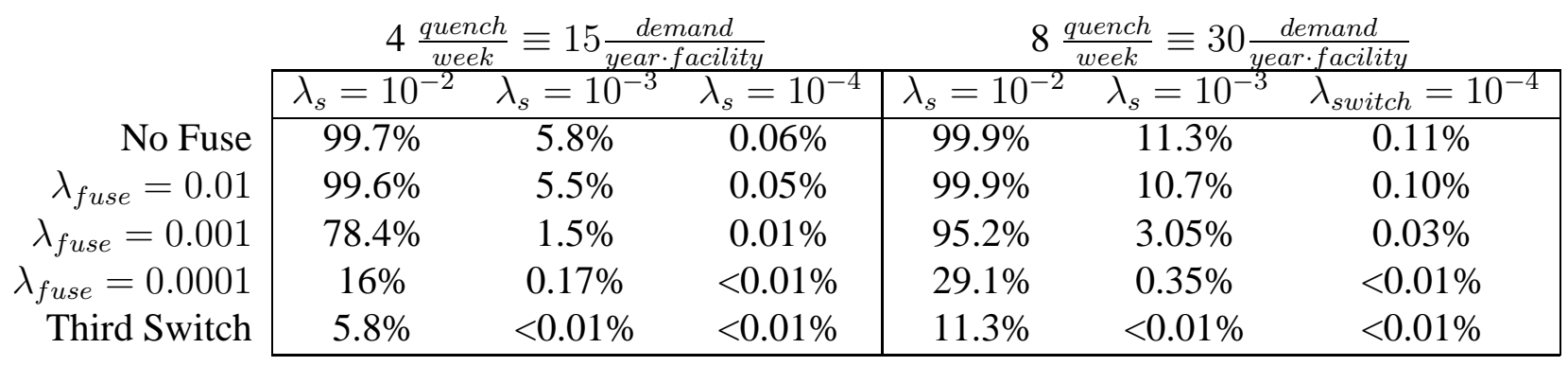

Table 9.6: Failure probability after 20 years of the 600 A energy extraction with maintenance after opening demand $\left(\lambda_{s} \equiv \lambda_{\text {switch }}\right)$.

\section{Safety Integrity Level}

Like for the main circuits, the safety integrity level is a very useful parameter to evaluate the necessity of implementing the controlled fuse on the 600 A energy extraction stations.

In Fig. 9.7 the SIL with and without the third switch is shown in function of the number of quenches per week and the expected switch failure rate. The SIL values become much more promising when the third fuse is included. It is important to remember that the option of firing heaters along the whole arc after a switch open failure is only applicable to the main magnets. There is not a back-up solution for failures in the $600 \mathrm{~A}$ facilities. Therefore, a switch open failure after a quench in the corrector circuits might lead to longer downtimes (around 1 month), which, acording to IEC-61508, is a Major failure (see Appendix C) that requires SIL 4. This can be assured only if a third switch is implemented.

\subsection{Conclusions about Maintenance}

It can be noticed for both the $13 \mathrm{kA}$ and the $600 \mathrm{~A}$ energy extraction facilities that usage of postmortem data is very important and fruitful even with scheduled monthly tests. This was not the case for the quench detectors and the quench heater power supplies, whose reliability was considerably improved by the post-mortem data when yearly tests were carried out, but it was rather useless if tests were make each month. This occurred because post-mortem data provided information only about the quenched elements, which seen the lower expected quench rate, was not comparable with the large number of subsystems tested when Quench Tests are sent to all the detectors of the machine. On the contrary, every quench implies testing one eighth of all the LHC energy extraction facilities.

It is rather plausible that each arc will quench at least once per month. If post-mortem data is taken into account and the extraction facilities of the subsector hosting the quenched element are set as good as new, the effect will be the same than carrying out scheduled monthly tests. 

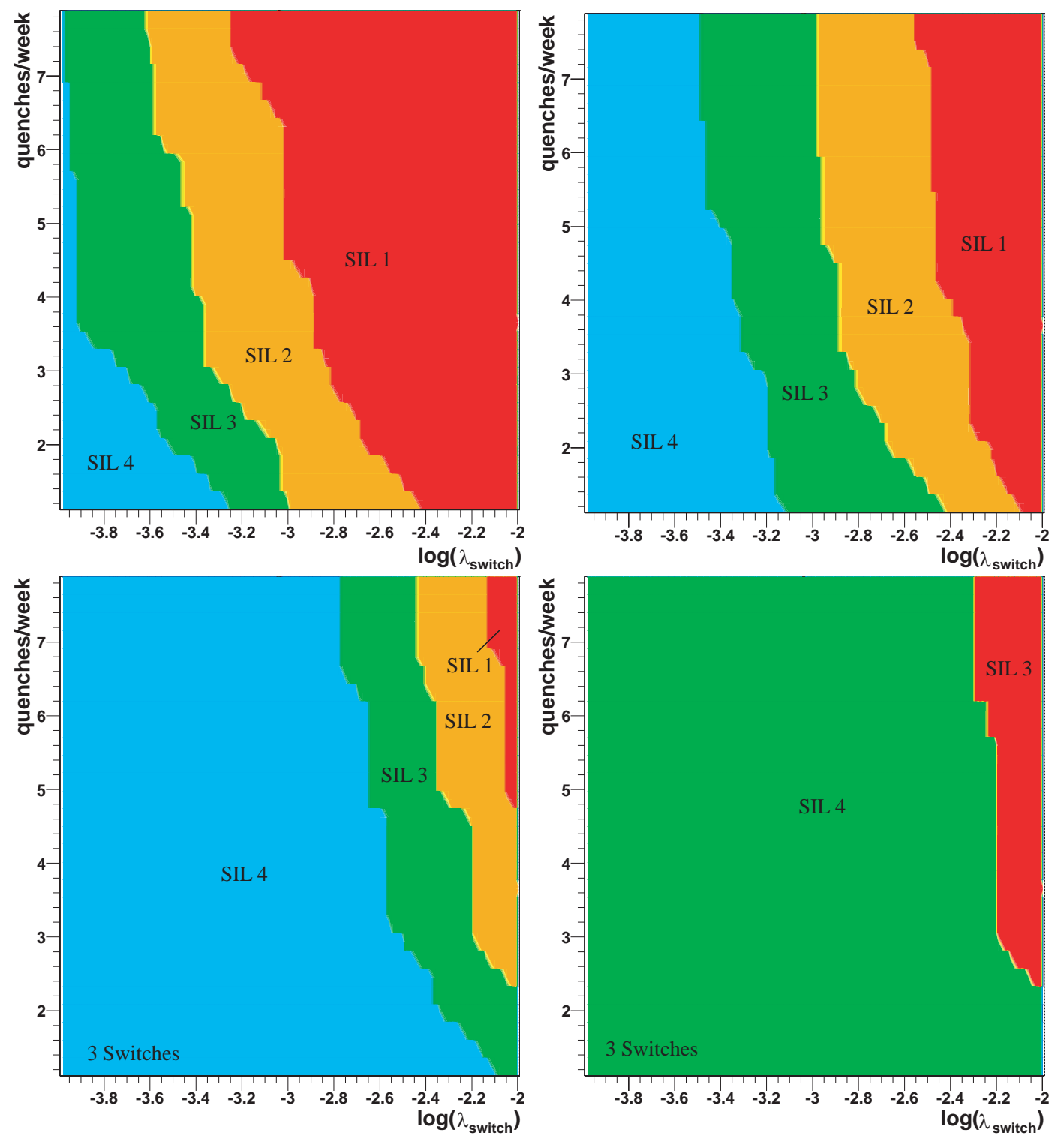

Figure 9.7: SIL for the 600 A energy extraction system without third fuse (up) and with it (down) for with maintenance carried out after each request (right) or yearly (left). 



\section{Chapter 10}

\section{The QPS and LHC Machine Dependability}

\subsection{Machine Interlock Systems}

It has been already explained in Section 4.5 the special characteristics of the LHC that give a crucial importance to the machine protection systems. They are in charge of protecting the beam and the hardware, and assisting to improve the machine operation and failure identification. Together with the Quench Protection System, the LHC is protected by the:

- Beam Dump System: in charge of extracting and extinguishing the two beams from the machine in a controlled way.

- Beam Loss Monitor System: controls the particle losses of the beam along the ring in order to prevent quenches, keep the integrity of superconductors, and reduce the dose to the components in the tunnel.

- Collimation System: reduces the losses around the arc.

- Access System: controls the accesses to the tunnel and impedes people to get close to the accelerator while it is running.

Although most of these systems might, in principle, be totally independent from each other (e.g. QPS and access control system), they must be coordinated and linked by a common structure: the Machine Interlock System. It is in charge of receiving the output information from the protection systems and, together with the data from other machine systems (e.g. experiments, vacuum, cryogenics, etc), generate the appropriate actions to ensure a reliable and safe performance of the accelerator. The machine interlock can be understood as the glue linking and structuring the subsystems [84].

Since magnets can be powered independently of the beam conditions, the machine interlock is divided into separate systems for powering and beam operation: the Powering Interlock System and the Beam Interlock System ${ }^{1}$. These systems are largely independent. No signal is sent from the beam to the power interlock and only one, in case of a power fault, from the powering to the beam system. Figure 10.1 shows the relationships between powering and beam interlocks and the main LHC subsystems.

\footnotetext{
${ }^{1}$ Since personnel safety has the highest priority, the access system is treated independently from the two interlock systems. Nevertheless, an interface between them is needed.
} 


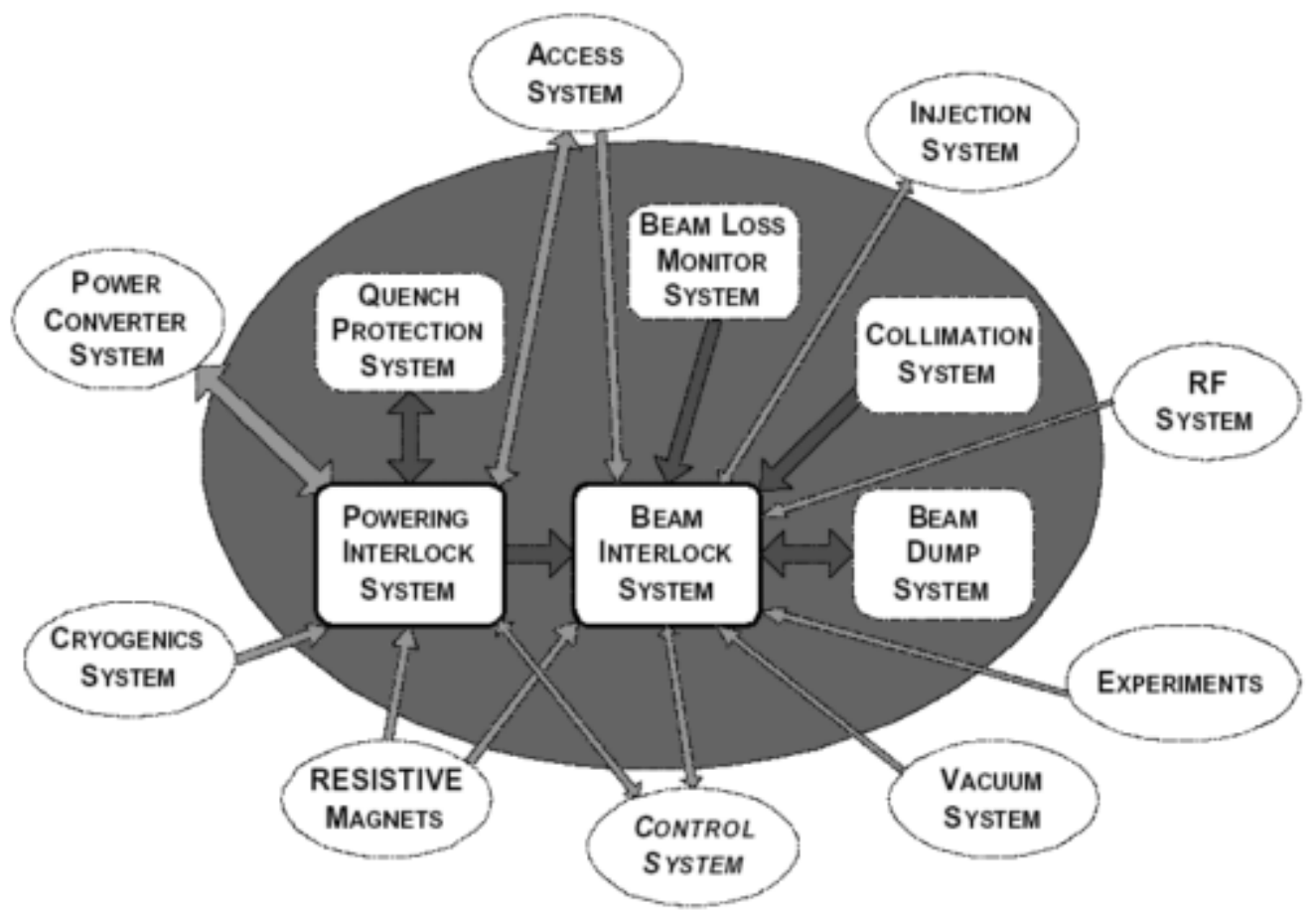

Figure 10.1: Relationships between the interlock and the machine subsystems. The systems dedicated to protection are within the grey area.

The Powering Interlock System allows for the powering of the magnets when certain conditions are met. It causes a safe dissipation of the energy stored in the magnet system in case of a quench or other events.

The Beam Interlock System allows beam injection when it is safe to accept beam, and requests a beam dump by the Beam Dump System if any unsafe scenario is detected.

A Post Mortem System records data from various systems in order to understand the cause of a failure leading to a beam dump or power abort.

The Quench Protection System is linked to the Powering Interlock and any action from the QPS that requires a response from the systems related to the beam will be sent to the Beam Interlock through the Powering Interlock System. Hence during this chapter we will analyse the links and interactions between the QPS and the Powering Interlock System only. A detailed explanation of the Beam Interlock System, its architecture and its relationships with the beam related subsystems can be found in [5] and [84].

\subsection{Powering Interlock System}

The high sectorisation of the LHC is a huge advantage from the machine protection point of view and has an important impact on the interlock architecture. The energy stored in the magnets of a cryostat must be discharged in case of a failure. However, the energy of the magnets in the other cryostats does not have to be extracted. Each cryostat (or a group formed by a few adjacent cryostats) is called a Powering Subsector with one Powering Interlock Controller or PIC. This allows powering all circuits in one subsector independently from the other electrical circuits. 
For each circuit, the Powering Interlock Controller has an interface to the power converter and the QPS. The power converters for the main dipoles and quadrupoles magnets in the arcs and the energy extraction stations for the quadrupoles are installed in the even points (see Figure 1.2). Since for the main dipoles there is an energy extraction facility at each end of the arc cryostat, the long arc cryostats will need two Powering Interlock Controllers, one on each side of the arc.

\section{Powering Interlock Actions}

The signals monitored by the powering interlock can be divided into: Signals for Power Abort and Signals for Power Permit.

The first group comprises the signals that are able to generate a circuit power abort. Although this will likely involve a beam dump, it will not be always the case since the failure of an electrical circuit will have severe or less severe consequences depending on the state of the accelerator and the kind of circuit failed. The concept of Interlock Classes is introduced in order to define the specific actions taken by the PIC software in case of a failure. All circuits within one class are handled with the same procedure:

- Interlock Class 1: The circuit is essential for beam operation, hence the beam is dumped. The energy in the circuit is large, therefore after a quench all the converters of the subsector are switched off and the energy extracted (48 circuits).

- Interlock Class 2: The magnets are essential but the energy is small. The beams are dumped but the other circuits of the subsector are left operating (580 circuits).

- Interlock Class 3: The magnets are not essential. The beam in not dumped and the circuits that not quenched are kept operating (866 circuits).

The Power Permit acts as a green light to power the circuits after a power abort. It cannot abort the beam run or the powering cycle and its removal during normal operation will not have any consequence until the next power abort or long machine shutdown. In a perfect LHC scenario the Power Permit should be given only once per year. The individual signals forming both groups are analysed below.

\section{Quench Loops}

Electrical circuits that include magnets with large stored energy are provided with a current loop that connects all the quench detectors and the discharge switches. There is one current loop for each main dipole circuit and another one shared by the two quadrupole circuits of an arc. The MQM, MQY and MQX magnets are also provided with quench loops.

The current loop has a very simple design in order to maximise its availability and avoid spurious triggers. In case of a quench the quench detector of the transited magnet will open the current loop via a relay. This will inform the equipment connected to the loop: the PIC and the energy extraction switches. The time between the detection of a quench and the breaking of the loop is about $20 \mathrm{~ms}$.

Current loops are also used for transmitting the power permission, powering failures and discharge. 


\section{Interfaces to the Beam Interlock System}

From each PIC two signals will be routed to the Beam Interlock System. They will inform about the status of the essential and the auxiliary circuits.

Any failure in the powering causes the corresponding signal to the beam interlock to get the failure state. An internal readable jumper in the Powering Interlock Controller determines whether the electrical circuit that has failed is essential for operating the beam. The definition of the LHC circuits that are essential for running the beam has not yet been done by the beam physics groups.

\subsection{Power Abort from QPS}

It has been demonstrated along this thesis that the quench protection system has a crucial impact on the LHC availability. It is the responsible of detecting and acting against dangerous scenarios that otherwise would lead to unacceptable economical and time costs. The Power Abort is the main link through which the QPS informs and activate most of their own and related subsystems.

\subsubsection{Power Abort Signals Between QPS, Power Converters and PIC}

The quench protection components that are able to stop the machine have been detailed along the different chapters of this thesis. Here we will focus on the interconnections between components and the required actions after the different machine failures.

The hardwired signals between the components in each electrical circuit and the PIC that are in charge of the power abort are listed in Table 10.1 .

\begin{tabular}{lccccc} 
Signal Name & Signal=1 & Signal=0 & From & To & Number \\
\hline QUENCH STATUS & No Quench & Quench & QPS & PIC & 1 per Circuit \\
POWERING FAILURE & PC OK & PC Failed & PC & PIC & 1 per Circuit \\
PC FAST ABORT & PC Permitted & PC Fast Off & PIC & PC & 1 per Circuit \\
PD DISCHARGE REQ. & PC OK & Fast Discharge & PC & PIC & 1 per Main PC and MQX. \\
\hline
\end{tabular}

Table 10.1: Hardwired signals between QPS, power converters and Powering Interlock Controllers generating a power abort.

\section{Circuit Quench}

The main actions of the QPS and the Powering Interlock after a quench in one magnet with Interlock Class 1 (arc dipoles and quadrupoles, decapole correctors, RQX, MQM and MQY) are:

- 1: The quench detector opens the quench loop of the circuit.

- 2: The energy of the circuit is extracted (if needed) by opening the energy extraction switches directly connected to the loop.

- 3: The PIC associated to the cryostat issues a PC Fast Abort.

- 4: The PIC issues a Discharge Request for the other main circuits of the cryostat. 
- 5: The rest of the circuits are discharged via a PC Fast Abort Signal.

For the rest of the circuits (Interlock classes 2 and 3) the steps 4 and 5 are not performed.

\section{Power Converter Failure}

The power converter sends a PC Discharge Request to the PIC if a fast discharge is required. The PIC activates the discharge switch for this circuit by opening the quench loop (in the case of the MQX circuit the quench heaters are discharged.).

If the failure requires a slow discharge the converter sends a Powering Failure signal to the PIC, which takes away the permission to power the circuit.

\section{Internal Failure of the Discharge Switches}

If two branches of one $13 \mathrm{kA}$ energy extraction facility open with high current, the other two will not stand the current for a long time, hence all the breakers will be automatically opened and the energy of the circuit discharged. A signal will be sent to the PIC through the quench loop interface.

\section{Switch Open Failure}

When required opening of the energy extraction breakers fails, the backup solutions will be one of the two explained in the previous chapter: firing of some selected heaters along the circuit in order to generate enough resistance, or triggering an explosive fuse in series with the failed facility. Both actions will be directly handled by the QPS, which will send the order of stopping the converter to the PIC by opening the quench loop.

\subsubsection{Power Abort Reliability}

The reliability of the QPS and the maintenance strategy followed will determine the QPS availability on demand (i.e. quench) and the number of unnecessary stops due to spurious protection triggers. The global maintainability will be treated below, here we will detail the channels of the QPS that affect on the power abort and thus on the machine availability.

Table 10.2 summarises the results of the previous chapters. The number of independent channels from the QPS to the powering interlock that are able to generate a power abort are listed together with the probability of not suffering any unavailability on demand after 20 years of LHC operation. The nominal reliability values explained in each chapter have been used.

In the case of monthly checks, the repairs are carried out just after the failure is detected (i.e. after post-mortem and after quench checks). The $600 \mathrm{~A}$ reliability result is the one with a third switch implemented in series.

The Quench Protection System probability of properly reacting to all the quenches suffered by the superconducting elements of the machine during the 20 years of LHC operation is above 90\%. This value is even larger (above 98\%) for the main magnets, which are the most hazardous elements.

The second part of the power abort reliability comprises the spurious triggers of the protection system. In the QPS the main source (and almost the only) of unneeded power aborts will be the false signals from the quench detectors. There might be also a few stops during the 20 years due to accidental openings of the energy extraction breakers. 


\begin{tabular}{|c|c|c|c|c|}
\hline & & Channels & Yearly Check & Monthly Check \\
\hline \multicolumn{5}{|l|}{ Quench Detectors } \\
\hline & DQQDI,T & 316 & 0.801 & 0.991 \\
\hline & DQQDG & 351 & 0.932 & 0.997 \\
\hline & DQQDL & 2016 & 0.412 & 0.991 \\
\hline & DQQDC & 1198 & 0.619 & 0.974 \\
\hline & & 3882 & 0.1904 & 0.9536 \\
\hline \multicolumn{5}{|l|}{ Heater Power Sup. } \\
\hline & Dipoles & 1232 & 0.772 & 0.997 \\
\hline & Quadrupoles & 536 & 0.977 & 0.999 \\
\hline & & 1768 & 0.754 & 0.996 \\
\hline \multicolumn{5}{|l|}{ Energy Extraction } \\
\hline & $13,000 \mathrm{~A}$ & 32 & 0.567 & 0.963 \\
\hline & $600 \mathrm{~A}$ & 176 & 0.987 & 0.999 \\
\hline & & 208 & 0.559 & 0.963 \\
\hline$\overline{\text { QPS }}$ & & $\begin{array}{ll}5858 \\
\end{array}$ & $\overline{0.08}$ & $\overline{0.914}$ \\
\hline
\end{tabular}

Table 10.2: QPS Channels to the powering interlock and global reliability (spurious triggered not considered).

It was seen in Chapter 7 that the number of false quenches strongly depends on the detection topology, the powering and the inspection strategy. The mean number of expected false quenches with redundant powering and monthly coherency checks estimated in that chapter was around 22 per year, thus around four days per year of needless downtime due to problems in the QPS.

The exchange of signals between the powering interlock and the QPS elements must be also highly reliable. They will also be fail-safe, and simple and reliable interface must be designed. The reliability analysis of the interlock-side components (e.g. relays, PIC, loops, etc) is outside the scope of this work, nevertheless, they are of crucial importance in order to keep the high reliable levels committed by the quench protection system.

\subsection{Power Permit and Maintainability}

Together with the Power Abort, the Power Permit is the tool that the QPS has (via the powering interlock) to prevent damage in the machine. Whereas the Power Abort is sent while the subsector is powered, the Power Permit is a flag that gives the go-ahead before powering. It is only considered by the Powering Interlock Controller for start-up and not during operation. Hence, the Power Permit in a subsector is only needed after a Power Abort of such subsector.

Maintainability as treated in this thesis is divided in inspections and repairs. Inspections can be made discretely or on-line, whereas repairs can be carried out only after a Power Abort. The Quench Protection System will inform to the Powering Interlock that time is needed to repair components or perform inspections (e.g. quench checks) by taking away the Power Permit.

The QPS baseline for giving or removing the Power Permit is to impede the powering of a subsector whose protection systems are not $100 \%$ available (including full redundancy). This goal 


\begin{tabular}{|c|c|c|c|c|c|}
\hline & & Check & Inspection & Repair & Affected \\
\hline \multirow[t]{8}{*}{ Quench Det. } & DQQDL & Quench Test & Monthly & Monthly & All \\
\hline & & Post-mortem & After Quench & Before PP & Quenched Magnet \\
\hline & & Coherency Test & On-line & Monthly & All \\
\hline & & & On-line & Before PP & Subsector \\
\hline & Other & Quench Test & Monthly & Monthly & All \\
\hline & & Post-mortem & After Quench & Before PP & Circuit \\
\hline & & Powering Test & On-line & Monthly & All \\
\hline & & & On-line & Before PP & Subsector \\
\hline \multirow[t]{2}{*}{ DQHDS } & & Discharge Test & Monthly & Monthly & All \\
\hline & & & After Quench & Before PP & Quenched Magnet \\
\hline \multirow[t]{6}{*}{ Extraction } & $13,000 \mathrm{~A}$ & Open Check & Monthly & Monthly & All \\
\hline & & Post-mortem & Quench & Before PP & Subsector \\
\hline & & False Opening & On-line & Monthly & All \\
\hline & & & On-line & Before PP & Subsector \\
\hline & $600 \mathrm{~A}$ & Open Check & Monthly & Monthly & All \\
\hline & & Post-mortem & Quench & Before PP & Subsector \\
\hline
\end{tabular}

Table 10.3: Global QPS maintainability strategy.

does not affect to possible failures that will not lead to an unprotected scenario but to a spurious trigger.

Table 10.2 shows that a good reliability level is reached when repairs in all the subsystems are performed every month. The several maintenance strategies applicable to each component were detailed and compared in the Chapters 7, 8 and 9. The goal now is to define a global maintenance strategy for the whole Quench Protection System.

The maintainability for the quench detectors cannot relay only on the post-mortem data obtained after each quench because the quench rate per magnet is too low (even with quench propagation) and the detectors would not be checked monthly, which could make a magnet stay at a dangerous failure state for too long. Therefore, scheduled monthly quench tests in every subsector are needed even if such subsector has not suffered any power abort since the last quench test.

The same applies to the other detector families and to the quench heater power supplies, since the quench test also triggers them.

The coherency and powering flags are monitored on line, hence after a Power Abort repairs can be carried out in all the detectors of the subsector hosting the quenched element. According to the quench rate predictions, it is rather possible that the eight arc subsectors will quench at least once per month. Repairs on the detectors with channels in safe failure (i.e. quench state) could be carried out before Power Permit is given again to that subsector. For the subsectors which have not experienced any Power Abort during the month, the scheduled downtime for carrying out the quench tests might me used to make coherency repairs.

The same principle can be applied to the energy extraction facilities. Although the inspection is not made on-line, the post-mortem data will now give information about all the facilities of the subsector after every quench, hence monthly repairs will be possible just using the downtime until next Power Permit. Nevertheless, if the quench rate in a subsector is not high enough, the downtime used for carrying out the quench tests can be used for performing opening checks of the switches. 
Summarising, from a global point of view, the Quench Protection System requires repairs before every Power Permit of the failed elements of such subsector. Their failure state will be known using the online tests and the post-mortem data. Moreover, monthly shutdowns will be needed in all the subsectors in order to carry out quench tests and, if needed, opening checks on the energy extraction breakers.

\subsection{RESQP During LHC Operation}

The huge number of components installed in the LHC, together with its repetitive structure will allow to have, after few months of operation, enough confident data about the reliability and operation parameters that have been used in this thesis for defining the hardware and the maintainability strategy.

RESQP has been extended in order to become a friendly-user tool for treating the reliability and operational data obtained during commissioning and nominal running, and help to decide whether any change has to be applied to the hardware or the maintenance strategy.

The code has to be simple enough for being useful during most of the LHC lifetime, since longer the experience, more helpful will be the program.

A very relevant example of RESQP utility during operation is its assistance to answer the open question of Chapter 7 about the worthiness of implementing a second power supply in the local quench detectors in order to reduce the number of false quenches. RESQP predicts around 10 false quenches per year from the DQQDL detectors if the powering is not redundant. After some months we should be able to realize whether this prediction is too pessimistic and to decide whether a second power supply is needed. RESQP extension will help on computing the new experimental failure rate and to evaluate the goodness of the investment.

The other subsystems and components of the QPS leave a very thin margin for carrying out hardware modifications. Nevertheless, the maintenance policy is totally flexible and it can be relaxed or strengthen according to the experience.

RESQP should be the seed of a wider code, which treats the reliability data from the different accelerator systems and components and gives statistical inferences for being used as feedback for the design, maintenance and operation decisions.

This kind of data storage and management is one of the goals defined by the accelerator reliability community met in Grenoble (France) in February 2002 [85]. 


\section{Conclusions and Outlook}

One of the key aspects to start-up with this thesis has been an analysis of the state of the art in the reliability of particle accelerators. We have seen that, although the design of small accelerators with industrial and medical applications, already addresses the issue of reliable performance, this is not the case for the large particle accelerators, whose reliability policy is only focused on their maintenance.

It has been demonstrated here that a design of the Large Hadron Collider which does not consider reliability and availability of the subsystems as essential as the maximal energy and luminosity will cause huge extra costs and will impede the machine to reach the expected integrated luminosity. Reliability analysis is hence a powerful and useful tool to improve the LHC performance and safety.

The development of RESQP, together with the reliability data obtained from CERN experiences and outside sources, allow a precise study of the optimum design and maintenance policy of the different protection systems needed for the LHC.

The experiments and simulations have shown that the Quench Protection System, when it is fully available, can assure the integrity of all the LHC superconducting elements. Nevertheless, the dimensions, complexity and expected lifetime of the LHC makes mandatory a deeper understanding of the subsystem failures that can set Quench Protection temporally unavailable. The most dangerous failure scenarios have been simulated and the conclusions have been used in order to determine the elements of the system whose failures would cause major damages and longer downtimes. The quench detectors, the quench heater supplies and the energy extraction facilities have been analysed and optimised using RESQP.

\section{Summary of the Results}

The design of the quench detectors has been optimised in order to minimise both the probability of missing a quench and, at the same time, the number of spurious triggers. The decision adopted for the local quench detectors is based on treatment of the quench signal by four independent channels whose outputs are linked in couples by two AND gates which are downstream combined by an OR gate (i.e. 2oo2or2oo2). If quench tests are carried out monthly, this topology will detect all quenches with a confidence level higher than $99 \%$. The other detector families will have a simpler 1oo2. With a $95 \%$ confidence level, it can be stated that no quench will be missed in the LHC over the 20 years of operation. The number of false quenches will strongly depend on the redundant powering. Due to budget reasons, it has been decided to install redundant power supplies for all the detector families except the local detectors. These will start working without redundant powering but the possibility for an upgrade will be kept and applied depending on the first months of LHC operation.

The design of the quench heater power supplies has been validated and the best maintenance policy has been defined. Although two tests are planned, charge voltage and discharge, only the 
second increases significatively the reliability. The data obtained after every quench has revealed to be as useful as the frequent discharge checks due to the high redundancy level of the quench heater supply banks. If this post-mortem information is taken into account and discharge tests are carried out every month, the probability of failure is below $0.4 \%$ and $0.1 \%$ for the dipoles and the quadrupoles respectively.

RESQP has assisted to evaluate the need of implementing a back-up opening element in series with the breakers in order to interrupt the current in case of a switch opening failure, namely controlled pyro-fuses. The computations show that, if checks and repairs (scheduled or after a Power Abort) are done every month, the reliability for the $13 \mathrm{kA}$ energy extraction facilities is between 96\%- 99\% without the fuse and above $99.9 \%$ with it. For the $600 \mathrm{~A}$ stations, the probability of not suffering any accident vanishes if a third switch is installed.

Finally the global Quench Protection System reliability after 20 years of LHC operation has been estimated: all the superconducting elements of the machine will be properly protected after any quench with a confidence level of about $90 \%$ if repairs of the known failed elements in an unpowered subsector are carried out before giving the Power Permit. Moreover monthly shutdowns will be needed in all the subsectors to perform scheduled tests and repairs.

\section{Outlook}

Although one of the first objectives of this work was to create a tool for assisting on the design of the Quench Protection System, RESQP has been conceived for being applied to any multi-state protection system. The philosophy, formulae and the code itself can be used not only for the quench protection, but for all the LHC different systems and subsystems related to the personnel and machine protection and safety. Some examples within the LHC project can be:

- Beam abort and dump systems.

- Beam loss monitoring (more than 1,000 beam loss detectors in the LHC).

- Personnel access to the tunnel supervision.

- Global Interlock System.

- Collimation System.

RESQP can also be used during the operation of the accelerator for validating the hardware decisions and redefining the different maintenance policies. RESQP will be a helpful tool for managing the reliability data obtained during operation and deciding whether changes are needed.

The developed tools can be useful in other accelerator projects (e.g. VLHC, CLIC) and also outside the high energy physics field (e.g. ITER).

I hope this study demonstrates the feasibility and utility of reliability studies in the early phase of an accelerator design and provides a tool to the scientific community. 


\section{Appendix A}

\section{Radiation Test Results}

All the electronic components planned to be used inside the tunnel have been tested under radiation environments. The devices whose performance is affected by the radiation are listed here. A detailed overview of the irradiation tests carried out at CERN and associated institutes can be found in [34].

\section{A.1 Voltage References}

Voltage references are needed for the charge detector circuit of the quench heater power supplies, and for all the detector families.

The design of such references is based on different technologies. The more precise types use a subsurface or buried Zener diode as an internal reference element, whereas the low cost types are usually based on bandgap technology.

During the radiation tests the current drain and the stability of the output voltage were subjected to measurement. The devices were exposed to $210 \mathrm{~Gy}$ with an average dose rate of $0.19 \mathrm{~Gy} / \mathrm{hour}$.

\begin{tabular}{lccl} 
Device & Accuracy & Drift & Technology \\
\hline LM4040 & $\pm 0.1 \%$ & $+0.19 \%$ & Zener/Bandgap \\
LT1236A & $\pm 0.05 \%$ & $-0.08 \%$ & Buried Zener \\
REF102 & $\pm 0.025 \%$ & $+0.005 \%$ & Buried Zener \\
AD581 & $\pm 0.05 \%$ & $+0.2 \%$ & Bandgap \\
MAX876 & $\pm 0.1 \%$ & $+2.15 \%$ & Bandgap \\
\hline
\end{tabular}

Table A.1: Influence of the radiation to the output voltage of different voltage references.

The outcome of the irradiation tests shows clearly that the different technologies result also in a different radiation tolerance. All devices based on the bandgap technology exhibited a significant increase of the output voltage, which is far beyond the tolerances given by the respective datasheets. In contrast to these results, both tested buried Zener devices showed a remarkable stability. However only the REF102 is fulfilling completely the technical specification. The current current consumption was not affected by the radiation in any of the tested devices. 


\section{A.2 Linear Voltage Regulators}

As the power consumption of the low voltage circuit is rather moderate, the corresponding power supplies have a classical design using linear voltage regulators. Three devices of the Motorola MC78xxCT/MC79xxCT have been tested. During the tests the current drain and the output voltage of each device was monitored. All devices had to supply a resistive load simulating the power consumption of the real application. The devices were exposed to $165 \mathrm{~Gy}$ and $214 \mathrm{~Gy}$ respectively.

\begin{tabular}{lcccc} 
Device & $V_{0}[\mathrm{~V}]$ & $\Delta V[\mathrm{mV}]$ & Rate $[\mathrm{mV} / \mathrm{Gy}]$ & $I_{\text {load }}[\mathrm{mA}]$ \\
\hline MCT7815CT_1 & 14.79 & -43 & -0.26 & 700 \\
MCT7815CT_2 & 14.990 & -13 & -0.08 & 40 \\
MCT7915CT & -15.18 & -64 & -0.30 & 750 \\
\hline
\end{tabular}

Table A.2: Linear voltage regulators submitted to radiation tests.

As a result, the current drain of all devices under test decreased slightly. The output voltage of the $+15 \mathrm{~V}$ and the $-15 \mathrm{~V}$ regulator decreased also with the accumulated dose. The lowest loaded device MCT7815CT exhibited the lowest deviation. All the tested devices show, total deviations within the tolerances given by the manufacturer.

\section{A.3 Bipolar Dual Timers}

The heater discharge power supplies comprise Phillips NE556N dual timers, which are used to generate the firing pulses for the thyristors and the timing sequence for a soft start circuit. Three samples of the NE556N-GBX0236-9722nK type were submitted to test. In each test cycle the current drain and the length of the generated pulse was measured. The results give, besides a slight decrease in the current drain of about $2 \mathrm{mu} A / \mathrm{Gy}$, no evidence of any radiation induced change of the device performance.

\section{A.4 Instrumentation Amplifiers}

The quench detectors are equipped with analog input stages, which are based on instrumentation amplifiers. The design of the quench detector is such that any adjustment of these inputs stages is a avoided. In consequence, gain and offset of the instrumentation amplifiers must exhibit sufficient stability during operation and exposure to radiation.

The performed tests show remarkable differences between various brands of instrumentation amplifiers, whereas the deviations within a batch rest rather small. The results (see Figures A.4) for the tested AD620 and INA141, show clear advantages for this later device. In addition, the offset voltage of all the INA141 amplifiers was found to increase, in first approximation, linearly with the dose. Linear fits five an average value of $0.20 \mathrm{mV} / \mathrm{Gy}$. As this value is regularly monitored during LHC operation. it can serve as a warning sign for a potential radiation damage of the device. 

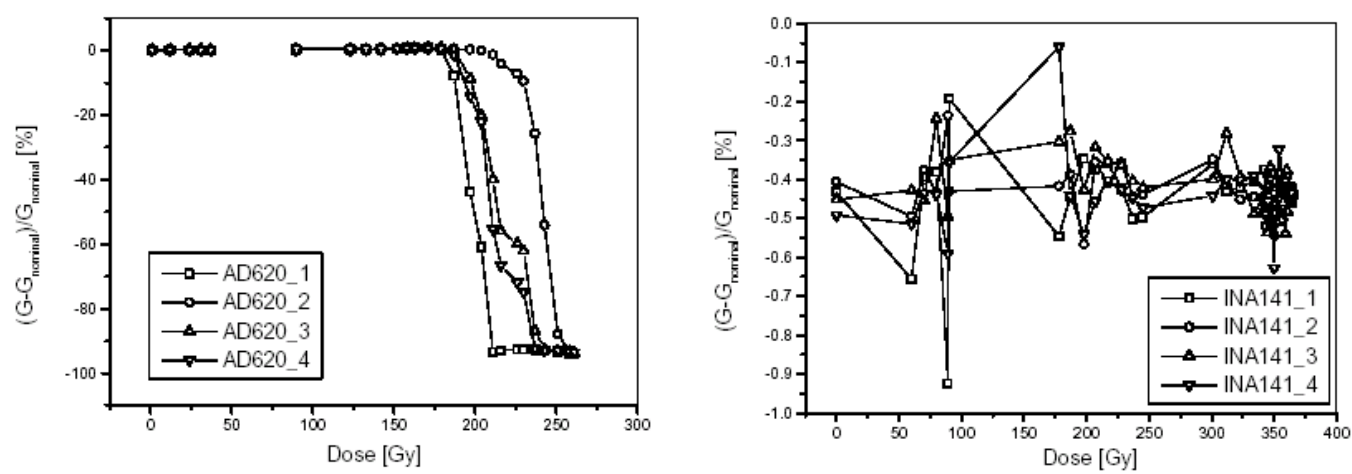

Figure A.1: Gain stability of the AD620 (left) and INA141 (right) isolation amplifiers.

\section{A.5 Switch Mode Power Supplies}

The local quench detectors require switch mode power supplies with $+5 \mathrm{~V}$ and $\pm 15 \mathrm{~V}$ outputs. Three batches of five units each of industrial grade from different manufacturers were submitted to test.

\begin{tabular}{lcccl} 
Device & $\begin{array}{c}\text { Dose } \\
{[\mathrm{Gy}]}\end{array}$ & $\begin{array}{c}\text { Dose Limit } \\
+5 \mathrm{~V}[\mathrm{~Gy}]\end{array}$ & $\begin{array}{c}\text { Dose Limit } \\
\pm 15 \mathrm{~V}[\mathrm{~Gy}]\end{array}$ & \multicolumn{1}{c}{$\begin{array}{c}\text { Failure } \\
\text { Mode }\end{array}$} \\
\hline A1 & 820 & 800 & 820 & \\
$\mathrm{~A} 2$ & 820 & 670 & 820 & $+5 \mathrm{~V}$ output \\
$\mathrm{A} 3$ & 820 & 660 & 820 & voltage starts \\
A4 & 820 & 650 & 820 & to decrease \\
A5 & 820 & 650 & 820 & \\
\hline B1 & 580 & 580 & 400 & Regulation of \\
B2 & 580 & 580 & 200 & $\pm 15 V$ stage \\
B3 & 580 & 580 & 190 & fails; output \\
B4 & 580 & 580 & 190 & voltage drifts \\
B5 & 580 & 580 & 190 & to zero \\
\hline C1-6 & 160 & 160 & 160 & No Failures \\
\hline
\end{tabular}

Table A.3: Switch mode power supplies.

Because of the limited test capacity, the five devices of brand $\mathrm{C}$ could only be tested up to $160 \mathrm{~Gy}$. In case of brand B, which also has been successfully tested by a different group at CERN, the manufacturer was able to trace down the failure to the component level (i.e. drift of a linear regulator in the $\pm 15 \mathrm{~V}$ stage) and proposed a modified design, which may be tested in the next radiation campaign.

\section{A.6 Data Acquisition}

Originally it was foreseen to use for the supervision of the LHC Protection System programmable logic controllers (PLC) equipped with analog and digital inputs and a PROFIBUS DP interface. 
Two PLC systems (PLC1 and PLC2) were submitted to test. The modules were exposed to 61 Gy and 32 Gy respectively.

Both tested systems failed after a few minutes of exposure to radiation indicating a strong sensitivity to single event effects. In addition, the power supply of the second PLC was broken during the irradiation.

The system has been substituted by a micro-controller of the ADuC812 type, which combines a 12-bit 8 channels ADC with an 80C51 core. The fieldbus coupler is based on the MICROFIP VY27257 ASIC and implements the WorldFip protocol. The radiation tolerant of the VY27257 has been already verified on detail [36].

The test was focused on the acquisition of analog signals for quench detectors and heater power supplies. The device transmitted data properly up to a total integrated dose of $650 \mathrm{~Gy}$, when the power supply broke down. Some of the acquired analog data, however, show significant deviations from their initial values, which are most probably caused by the amplifiers used for the buffering of the I/O channels to the ADuC812.

\begin{tabular}{lccc}
$\begin{array}{l}\text { ADC Input } \\
\text { Channel }\end{array}$ & $\begin{array}{c}\text { Nominal } \\
\text { Voltage [V] }\end{array}$ & $\begin{array}{c}\text { Initial } \\
\text { Values [V] }\end{array}$ & $\begin{array}{c}\text { Last Value } \\
\text { Transmitted [V] }\end{array}$ \\
\hline ADC1 & 2.5 & 2.495 & 2.495 \\
ADC2 & 2.0 & 1.999 & 1.984 \\
ADC3 & 1.5 & 1.499 & 1.558 \\
ADC4 & 1.0 & 0.999 & 1.058 \\
ADC5 & 0.5 & 0.497 & 0.572 \\
ADC6 & 0.2 & 0.205 & 0.178 \\
ADC7 & 0.5 & 0.516 & 0.489 \\
\hline
\end{tabular}

Table A.4: ADuC812 data acquisition system.

\section{A.7 Quench Heater Power Supplies}

The individual electronic components and subsystems of the quench heater power supplies have been extensively tested during the last five years [36]. In the last year, the five power supply have been tested in order to validate the required radiation tolerance of $200 \mathrm{~Gy}$. The samples have been taken out from different preseries production batches. The devices were operated under nominal conditions performing charge-discharge cycles. During the test, the voltage across the capacitor bank was monitored.

As was already seen in previous irradiation campaigns, the useful life of the power supply under radiation is mainly limited by the two thyristors used for the discharge of the capacitor bank. In the tested preseries devices three different brands of thyristors were installed, all of $1.8 \mathrm{kV}$ 80 A rating. All thyristors suffered from defects induced by radiation, which affected their proper function after exposures to doses between 50 and $400 \mathrm{~Gy}$. Depending on the type of thyristor either the firing was inhibited or it was destroyed during the discharge. The results are summarised in Table A.7. Despite the problems with the thyristors, no other radiation induced failures were revealed by the tests. 


\begin{tabular}{lccc} 
Device & $\begin{array}{c}\text { Thyristor } \\
\text { Type }\end{array}$ & $\begin{array}{c}\text { Dose Limit } \\
{[\mathrm{Gy}]}\end{array}$ & $\begin{array}{c}\text { Failure } \\
\text { Type }\end{array}$ \\
\hline 1 & $\mathrm{~A}$ & 160 & Firing inhibited \\
1 & $\mathrm{~B}$ & 140 & Firing inhibited \\
1 & $\mathrm{C}$ & 380 & Short after discharge \\
2 & $\mathrm{~B}$ & 50 & Firing inhibited \\
3 & $\mathrm{C}$ & 400 & Short after discharge \\
4 & $\mathrm{C}$ & 150 & Not radiation induced \\
5 & $\mathrm{C}$ & 420 & Short after discharge \\
\hline
\end{tabular}

Table A.5: Irradiation tests of the quench heater power supplies.

\section{Stress and Ageing Tests}

Six electrolytic capacitors of $4.7 \mathrm{mF}$ and $500 \mathrm{~V}$ of different manufacturers (Elwa, Trobo, Leclanché. Rifa, BCH Aerovox and Siemens) have been tested in collaboration with the Université de Lyon.

The capacitors from Trobo and Elwa have been rejected due to the following early failures:

- Elwa: a component explodes under $500 \mathrm{~V}$ and $85^{\circ} \mathrm{C}$ after 1 hour of ageing.

- Trobo: the lid of two components are melt after 450 hours.

Two capacitors from BHC showed leak of the electrolyte through the safety lid after 4,000 $\mathrm{h}$ and $1300 \mathrm{~h}$ hours. The Rifa capacitors changed the external aspect (colour) probably due to electrolyte leak through the aluminium box.

Several conclusions can be taken from the charge-discharge test:

- The capacitance is not affected by the cycles.

- The series resistance increases specially for the Rifa capacitors.

- The leakage current notably increases.

The main consequences of this ageing will mainly affect the connections of the capacitors and not on the drying of the electrolyte. 



\section{Appendix B}

\section{Reliability Data From Life Testing}

The mean time between failure (MTBF) at different confidence levels can be obtained by accelerated life testing. We consider that during the useful lifetime of the tested components there are no systematic defects or problems causing a high early failure rate or failure associated with ageing. Failures during this period result from random causes. We have seen that, under this consideration, the probability density function of the time to failure can be expressed as:

$$
f(t)=\alpha e^{-\alpha t}
$$

The expected value of $f(t)$ is the MTBF thus $1 / \alpha$. An estimator of this value can derived using the maximum likelihood method with the function $\mathrm{f}(\mathrm{t})$. Let's suppose we run a life test starting with $N$ parts and $r$ experience failures. The joint probability density function describing the life test results is given by the product of the probabilities that each failure occurred when it did. We refer at this probability density function as $L(\alpha, t)$ :

$$
L(\alpha, t)=\alpha^{r} e^{-\alpha \sum_{i=1}^{r} t_{i}}
$$

It is implicit to this derivation that the life test is terminated at the $r-t h$ failure and the dimension of $t$ is the device cdothour. This method involves adding one failure to the total observed failures. This assures the requirement for termination on the $r-t h$ failure is satisfied, as well as allows calculation of the MTBF even when no sample has failed.

An estimator of $1 / \alpha$ may be the value of $\alpha$ that maximises the function $L(\alpha, t)$. This is accomplished by taking the partial derivative of $\ln [L(\alpha, t)]$, hence

$$
\begin{gathered}
\ln [L(\alpha, t)]=r \ln (\alpha)-\alpha \sum_{i=1}^{r} t_{i} \\
\frac{\partial \ln [L(\alpha, t)]}{\partial \alpha}=\frac{r}{\alpha}-\sum_{i=1}^{r} t_{i} \\
\frac{r}{\tilde{\alpha}}=\frac{\sum_{i=1}^{r} t_{i}}{r}
\end{gathered}
$$


where $\tilde{\alpha}$ to indicate the following approximation:

$$
\frac{1}{\tilde{\alpha}}=\frac{\sum_{i=1}^{r} t_{i}}{r}
$$

This equation shows that $1 / \alpha$ can be estimated by dividing the accumulated test time for all the tested devices by the number of failures, which agrees with the original definition of MTBF.

The calculation of confidence intervals using the chi-square $c h i^{2}$, can be understood from a give random variable for time to failure, $\mathrm{T}$, which has the distribution in Eq. B.1, then the random variable $V$ described by

$$
V=2 \alpha \sum_{i=1}^{r} t_{i}
$$

is distributed $\chi^{2}$ with $2 r$ degrees of freedom. Therefore, for a specified confidence level $\zeta$

$$
2 \alpha \sum_{i=1}^{r} t_{i}>\chi^{2}(\zeta, 2 r)
$$

and the upper confidence limit for the Mean Time Between failure becomes

$$
\frac{1}{\alpha}<\frac{2 \sum_{i=1}^{r} t_{i}}{\chi^{2}(\zeta, 2 r)}=M T B F
$$

which, with $r$ being the number of failures plus one, is the actual formula we use for the MTBF. 


\section{Appendix C}

\section{Safety Integrity Level}

Next tables show the Safety Integrity Levels (SIL) suggested by IEC-61508 depending on the failure probability of the protection system and the initiator likelihood.

Average probability of failure to perform

\begin{tabular}{cc} 
SIL & its design function on demand $\left(F P P D_{a v}\right)$ \\
\hline 4 & $10^{-5}<\operatorname{Pr}<10^{-4}$ \\
3 & $10^{-4}<\operatorname{Pr}<10^{-3}$ \\
2 & $10^{-3}<\operatorname{Pr}<10^{-2}$ \\
1 & $10^{-2}<\operatorname{Pr}<10^{-1}$ \\
\hline
\end{tabular}

Table C.1: SIL with low demand mode of operation (IEC-61508).

\begin{tabular}{cc} 
SIL & Probability of a dangerous failure per hour \\
\hline 4 & $10^{-9}<\operatorname{Pr}<10^{-8}$ \\
3 & $10^{-8}<\operatorname{Pr}<10^{-7}$ \\
2 & $10^{-7}<\operatorname{Pr}<10^{-6}$ \\
1 & $10^{-6}<\operatorname{Pr}<10^{-5}$ \\
\hline
\end{tabular}

Table C.2: SIL with high demand or continuous mode of operation (IEC-61508).

\begin{tabular}{lcccc}
\multirow{2}{*}{$\begin{array}{c}\text { Event } \\
\text { Likelihood }\end{array}$} & \multicolumn{4}{c}{ Consequence } \\
\cline { 2 - 5 } & Catastrophic & Major & Severe & Minor \\
\hline Frequent & SIL 4 & SIL 3 & SIL 3 & SIL 2 \\
Probable & SIL 3 & SIL 3 & SIL 3 & SIL 2 \\
Occasional & SIL 3 & SIL 3 & SIL 2 & SIL 1 \\
Remote & SIL 3 & SIL 2 & SIL 2 & SIL 1 \\
Improbable & SIL 3 & SIL 2 & SIL 1 & SIL 1 \\
Negligible & SIL 2 & SIL 1 & SIL 1 & SIL 1 \\
\hline
\end{tabular}

Table C.3: Minimum Safety Integrity Level required (IEC-61508). 


\begin{tabular}{lcc} 
Category & Description & Yearly frequency level \\
\hline Frequent & Event occurs very likely & $>1$ \\
Probable & Event is likely to occur & $10^{-1}-1$ \\
Occasional & Event possible and expected & $10^{-2}-10^{-1}$ \\
Remote & Event possible but not expected & $10^{-3}-10^{-2}$ \\
Improbable & Event unlikely to occur & $10^{-4}-10^{-3}$ \\
Negligible & Event extremely unlikely & $10^{-5}-10^{-4}$ \\
\hline
\end{tabular}

Table C.4: Frequency categories.

\begin{tabular}{lcccc}
\multirow{2}{*}{ Category } & \multicolumn{2}{c}{ Injury to Personnel } & \multicolumn{2}{c}{ Damage to Equipment } \\
\cline { 2 - 5 } & Criteria & Fatalities & CHF Loss & Downtime \\
\hline Catastrophic & Fatalities & $\geq 1$ & $>510^{7}$ & $>6$ months \\
Major & Very Serious Injuries & 0.1 & $10^{6}-510^{7}$ & 20 days -6 months \\
Severe & Serious Injuries & 0.01 & $10^{5}-10^{6}$ & $3-20$ days \\
Minor & Minor Injuries & 0.001 & $0-10^{5}$ & $<3$ days \\
\hline
\end{tabular}

Table C.5: Consequence categories. 


\section{Bibliography}

[1] U. Amaldi et al. Consistency Checks of GUT's with LEP Data. CERN-PPE/91-190, 1991.

[2] The LHC Study Group. LHC the Large Hadron Collider - Conceptual Design Vol.II. CERN/AC/95-05, October 1995.

[3] R. Schmidt. Accelerator Physics and Technology of the LHC. CERN Yellow Report 99-01, 1998.

[4] LHC Project Document LHC-PM-MS-0005, rev 1.4, April 2002.

[5] R. Schmidt and A. Vergara Fernandez. Machine Protection and Interlock System for the LHC. In Accelerator Reliability Workshop, Grenoble, February 2002.

[6] W.R. Ansorge. Reliability, Maintainability and Safety in Scientific and Technical Projects. CERN Academic Training Lecture, 2000.

[7] C. Garion and B.Skoczen. Reliability Oriented Optimum Design of the LHC Interconnections. CERN LHC Project Note 245, 2000.

[8] P. Schmuser. Superconductivity. In CERN Accelerator School. Superconductivity in Particle Accelerators, Hamburg, May 1995.

[9] H.K. Onnes. Akad. van Wetenschappen, 1911.

[10] W. Meissner and R. Ochsenfeld. Naturwissenschaften, 1933.

[11] J. Bardeen et al. Phys. Rev., 1957.

[12] V.L. Ginzburg and L.D. Landau. Sov. Phys., 1950.

[13] J.G. Bednorz and G.M. Muller. Possible high $T_{c}$ Superconductivity in $B a-L a-C u-O$ system. Zeitschrift fur Physic B, 1986.

[14] F. London and H. London. Z. Phys., 1935.

[15] M.N. Wilson. Superconducting Magnets. Oxford Science Publications, 1983.

[16] A. Ballarino. Application of High Temperature Superconductors for Accelerator Magnets. In Proceedings of EPAC'2000, Vienna, 2000.

[17] K.H. Mess et al. Superconducting Accelerator Magnets. World Scientific, 1996.

[18] F. Sonnemann. Resistive Transition and Protection of LHC Superconducting Cables and Magnets. PhD thesis, RWTH, Aachen, 2001. 
[19] M. Calvi. Quench Propagation in the LHC Superconducting Busbars. Master's thesis, Politecnico di Torino, 2000.

[20] D.R. Tilley and J. Tilley. Superfluidity and Superconductivity. Institute of Physics Publishing Ltd, 1990.

[21] J.G. Weisend. Introduction to Cryogenic Engineering. CERN Academic Training Programme, 2001.

[22] S. Redaelli. Analysis of the Magnetic Field Perturbations in Dipoles and Quadrupoles of the Large Hadron Collider. Master's thesis, Universita degli Studi di Milano, 2000.

[23] Web page http://lhc.web.cern.ch/lhc/.

[24] A. Yamamoto et al. Inner Triplet Quadrupole MQXA. Functional Specification LHC-MQXAES-0001, November 2001.

[25] J. Kerby and M. Lamm. Inner Triplet Quadrupole MQXB. Functional Specification LHCLQX-ES-0002, April 2001.

[26] S. Plate. LBRC Cryo-Assemblies - D2 Dipoles. Interface Specification LHC-LQXA-ES-3.0, January 2001.

[27] A. Ballarino. HTS Current Leads for the LHC Magnet Powering System. CERN LHC Project Report 608, October 2002.

[28] M. Bona et al. Cryogenic and Vacuum Sectorisation of the LHC arcs. CERN LHC Project Report 60, 1996.

[29] F. Rodriguez-Mateos et al. The Protection System for the Superconducting Elements of the Large Hadron Collider at CERN. Particle Accelerator Conference, 1999.

[30] F. Sonnemann et al. Quench Process and Protection of LHC dipole magnets. CERN LHC Project Note 184, 1999.

[31] R. Denz and D. Hagedorn. Experimental Analysis and Modeling of the Electrical and Thermal Transients of the Diode-By-pass for the LHC-Magnet Protection at Cryogenic Temperature. CERN LHC Project Report 268, 1999.

[32] R. Schmidt and F. Sonnemann. Quench Simulations of Superconducting Elements in the LHC Accelerator. Cryogenics, 40, 2000.

[33] F. Rodriguez-Mateos et al. General Parameters for Energy Extraction of the LHC Superconducting Circuits, September 2002.

[34] A. Vergara. Irradiation Tests on Electronic Components for the Protection System of the Superconducting Elements of the Large Hadron Collider at CERN. Master's thesis, ETSEIB, Universitat Politècnica de Catalunya, March 2000.

[35] G.R. Stevenson et al. Estimated Dose to Components in the Arcs of the LHC due to BeamLoss and Beam-Gass Interactions. CERN LHC Project Note 184, 1995. 
[36] A. Vergara et al. Irradiation Tests on COTS Used in the Protection System of the LHC Superconducting Elements. In RADECS'2000 European Workshop on Radiation Effects on Components and Systems, Université Catholique de Louvain-la-Neuve, Belgium, September 2000.

[37] International Standard IEC. 60050-1.

[38] D. Sornette. Critial Phenomena in Natural Sciences. Springer, 2000.

[39] A. Birolini. Reliability Engineering: Theory and Practice. Springer, 1999.

[40] T. Aven. Reliability and Risk Analysis. Elsevier Science Publishers, 1992.

[41] W.E. Vesely et al. Fault Tree Hand-book. U.S. Nuclear Regulatory Commission, 1991.

[42] R. Durret. Probability: Theory and Examples. Duxbury Press, 1996.

[43] E.J. Dudewicz and S.A. Mishra. Modern Mathematical Statistics. Wiley New York, 1988.

[44] US Department of Defense. Reliability Prediction of Electronic Equipment, 1995. Military Handbook MIL-HDBK-217F.

[45] C. Piaszczyck and M. Rennich. Reliability Survey of Accelerator Facilities. In Maintenance and Reliability Conference Proceedings, Knoxville, May 1998.

[46] C. Piaszczyck. Operational Experience at Existing Accelerator Facilities. In NEA Workshop on Utilisation and Reliability of High Power Proton Accelerators, Mito, Ocotober 1998.

[47] L.C. Cadwallader and T. Pina. Progress Towards a Component Failure Rate Data Banck for Magnet Fusion Safety. In International Topical Meeting on Probabilistic Safety Assessment, August 1999.

[48] K. Lauridsen et al. Assessment of the Reliability of Robotic Systems for Use in Radiation Environments. Reliability Engineering and Safety Systems, 53, 1996.

[49] A. Fritz and B. Bertsche. Algorithms for the Monte Carlo Simulation of the Reliability and Availability of Mechanical Systems. In ESREL 2001, Torino, September 2001.

[50] Y. Jongen. How to Design Medical Accelerator Systems for Reliability and Availability of Mechanical Systems. In Accelerator Reliability Workshop, Grenoble, February 2002.

[51] NASA-GFSC. Radiation Effects and Analysis. Washington, 1999.

[52] M. Stamatelatos. Risk Assessment at NASA: Vision, Status and Plans. Risk Management Colloquia, May 2001.

[53] IEC 61508. International Standard, first edition, May 2000.

[54] R. Schmidt. Private Communication.

[55] CERN Engineering Specification, General Parameters for Equipment Installed in the LHC. LHC-PM-ES-0002, 1999. 
[56] R. Schmidt et al. MachineProtection System for the LHC: Architecture of the Beam and Powering Interlock Systems. CERN LHC Project Report 521, December 2001.

[57] A. Saltelli et al. Sensitivity Analysis. Wiley Series in Probability and Statistics, 2000.

[58] A. Saltelli et al. A Quantitative, Model Independent Method for Global Sensitivity Analysis of Model Output. Technometrics, 41, 1999.

[59] P. Jarron. Radiation Tolerant Electronics for the LHC experiments. CERN, Geneva, 1998.

[60] K.A. Label. Single Event Effect Criticality Analysis. NASA Headquarters/Code QW, Washington, 1996.

[61] G.R. Stevenson and J.M. Zazula. The Estimation of Radiation Doses, Fluences, and Spectra from FLUKA Code Simulations of Particle Cascades Induced by Beam Losses on LHC Dipoles. CERN/TIS-RP/IR/92-30, 1992.

[62] R. Rausch and M. Tavlet. On-line Radiation Test Facility for Industrial Equipment Needed for the LHC at CERN. CERN, 1999.

[63] C.A. Fynbo. Qualification of the Radiation Environment in the TCC2 Experimental Test Area. CERN LHC Project Note 235, September 2000.

[64] S. Dowling. Chatacterising Systems and Components to Total Dose. In 5th Radecs Conference, Maine et Loire, France, 1999.

[65] R. Denz et al. Irradiation Tests at Cryogenic Temperatures on Diffusion Type Diodes for the LHC Superconducting Magnet Protection. CERN LHC Project Report 277, July 1998.

[66] R. Denz and F. Rodriguez Mateos. Radiation Tolerance of Components Used in the Protection System of the LHC Superconducting Elements. In RADECS'2002 European Workshop on Radiation Effects on Components and Systems, Padova, Italy, September 2002.

[67] F. Bordry et al. The LHC Magnet String Programme: Status and Future Plans. In PAC'99, New York, April 1999.

[68] L. Serio. Private Communication.

[69] F. Rodriguez D. Hagedorn. Modelling of the Quenching Processes in Complex Superconducting Magnet Systems. IEEE Trans. Magn., (28), 1992.

[70] F. Rodriguez-Mateos et al. Quench Protection Test Results and Comparative Simulations on the First 10 meter Prototype Dipoles for the Large Hadron Collider. In MT14, Tampere, June 1995.

[71] S. Russenschuck et al. ROXIE Routine for the Optimisation of Magnet X-Sections, Inverse Field Calculations and Coil End Designs. CERN Yellow Report 99-01, 1998.

[72] A. Danner and F. Sonnemann. Review of Quench Simulations for the Protection of the LHC Main Dipole Magnets. LHC ICP Internal Note 00-199, 1999.

[73] F. Rodriguez Mateos. Voltage Withstand Levels for Electrical Isulation Tests. LHC-PM-ES$0001,1999$. 
[74] A. Vergara. DQQDL Power Supplies: Discounted Cash-Flow Analysis. LHC ICP Internal Note, April 2002.

[75] R. Denz. Private communication, 2002.

[76] CERN Directorate Service Unit. Private Communication, February 2002.

[77] A. Ijspeert. Private Communication, February 2002.

[78] A. Roshald. Tests of the 600 A Controllable Fuses. Technical report, NIIEFA, SRC "SINTEZ", January 2003.

[79] J.D. Kalbfleisch and R.L. Prentice. The Statistical Analysis of Failure Time Data. John Wiley \& Sons, 1980.

[80] J.F. Lawless. Statistical Models and Methods for Lifetime Data. John Wiley \& Sons, 1982.

[81] A.A. Salvia and R.C. Bollinger. On Discrete Hazard Functions. IEEE Transactions on Reliability, R-31, December 1982.

[82] C. Bracquemond et al. Modeling the Ageing of Non Reparaible Systems in Discrete Time. In ESREL 2001, Torino, September 2001.

[83] K. Dahlerup-Petersen. Private communication, 2003.

[84] F. Bordry et al. Machine Protection for the LHC: Architecture of the Beam and Powering Interlock Systems. LHC Project Report 521, December 2001.

[85] Reliability Accelerator Workshop, Grenoble, February 2002. 



\section{List of Figures}

1.1 LHC cell layout: the six main dipole magnets, two lattice quadrupoles and correctors. 8

1.2 Layout of the Large Hadron Collider. . . . . . . . . . . . . . . . . . . . . . . . 9

2.1 Magnetisation of a type II superconductor as a function of the applied magnetic field. 13

2.2 Critical surface of the NbTi superconductor used in the LHC. . . . . . . . . . . . . 14

2.3 Cross section of the LHC main dipole strand (left) with NbTi filaments (dark) embedded by a copper stabiliser (grey). The Rutherford cable (right) is used for the outer layer of the same magnets. . . . . . . . . . . . . . . . . . . 16

2.4 Copper heat capacity in function of the temperature (left) and copper resistivity for different RRR in function of the temperature (right). . . . . . . . . . . . . . . 19

2.5 Voltage across an LHC main dipole after a quench. . . . . . . . . . . . . . . . 22

3.1 Twin-aperture LHC dipole magnet cross section, 1: heat exchanger pipe, 2: superconducting bus bars, 3: superconducting coil, 4: beam screen, 5: vacuum vessel, 6: radiation screen, 7: shrinking cylinder, 8: thermal shield, 9: non-magnetic collars, 10: iron yoke. . . . . . . . . . . . . . . . . . . 26

3.2 Electrical connection of the dipoles in one half cell. AP1 and AP2 make reference to the two apertures (beam tubes) and LOW and UP to the upper and lower coil. . . 27

3.3 Electrical connection of the MQ quadrupoles in one LHC cell. . . . . . . . . . . 28

3.4 Schematic layout of an insertion for the high luminosity experiment [3] . . . . . . 28

3.5 Schematic diagram of the busbar cross-section for the LHC main dipole and arc quadrupole magnets. . . . . . . . . . . . . . . . . . 30

3.6 HTS current lead [27] (LTS: low temperature superconductor, HTS: high temperature superconductor). . . . . . . . . . . . . . . 31

3.7 Schematic view of the dipole coils with the corresponding high and low field quench heater strips. . . . . . . . . . . . . . . . . . . 34

3.8 Energy extraction location for the main dipole circuits. . . . . . . . . . . . 35

4.1 Qualitative behaviour of the failure rate function. Bath-tube curve. . . . . . . . 43

4.2 Component $i$ illustrated by a block. . . . . . . . . . . . . . . . . . . 46

4.3 System function represented by a reliability block diagram. . . . . . . . . . . . . . 46

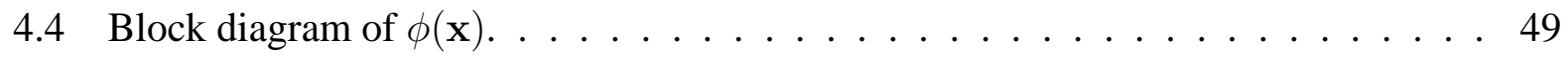

4.5 Redundancy at system level (left) and redundancy at component level (right). . . . 50

5.1 Scatter plots of 630 sampling points in a two-factor case with unconmensurate (left) and non-unconmensurate (right) frequencies. . . . . . . . . . . . . . 69

5.2 Sensitivity of maximum number of lost quadrupoles due to quench heater power supply unavailability (90\% confidence level) to component failure rate and quench rate with post-mortem information considered (right) and not considered (left). . . 70 
5.3 RESQP execution steps (SF: Safe failures, DF: Dangerous failures, MF: Main

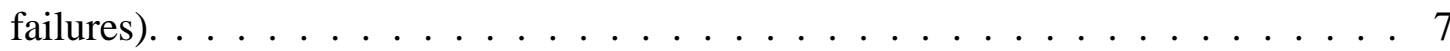

6.1 Simulated distribution along a regular cell of the total dose after 10 years of operation [34].

6.2 Excess of base current for a NPN discrete transistor against the dose rate after a 520 Gy irradiation [64] . . . . . . . . . . . . . . . . . . . . .

6.3 The LHC Test String-2 assembled in the SM18 building at CERN. The two individual magnet test benches can be also seen. . . . . . . . . . . . . . . . . . 82

6.4 String-2 electrical feedbox with the $600 \mathrm{~A}$ and $13 \mathrm{kA}$ HTS current leads mounted. . 83

6.5 First powering of the diode (left) and the quadrupole (right) circuits of the String-2. 84

6.6 Provoked quench in a String-2 (first phase) dipole which propagates to the other two. 85

6.7 Simulated dipole circuit $\left(R_{\text {Lead }}=R_{\text {BusBar }}=20 \mu \Omega, \sum L_{\text {Chain }}=16.4322 \mathrm{H}\right.$, $\left.\sum R_{E E}=150 m \Omega\right) \ldots \ldots \ldots \ldots \ldots \ldots \ldots \ldots \ldots \ldots \ldots \ldots \ldots \ldots \ldots \ldots$

6.8 Simulation times $\left(t_{t h}\right.$ :time to reach detection threshold, $t_{h d}$ : delay from heater quench since detection, $t_{q d}$ :total detection time, $t_{p a}$ : time to power abort, $t_{\text {switch }}$ : time to open the switches, $t_{\text {heater }}$ : heater quench delay from quench occurrence).

6.9 QUABER results for a properly protected dipole circuit. . . . . . . . . . . . . . . . 9

6.10 Up: Maximum temperature (light) and quench load (dark) for diode in open circuit; Down: magnet voltage (light) and current (dark); Nominal values are represented

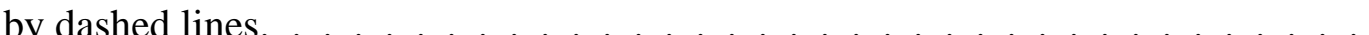

6.11 Up: Hot spot temperature and voltage along half cell for different turn-on voltages of the cold diode.

Hot spot temperature (left) and magnet voltage (right) in nominal condition (dark) and short-circuited diode (light). . . . . . . . . . . . . . . . . . . . . 94

6.13 Magnet voltage (light) and arc current (dark) after the failure of one energy extraction and diode damage due to the slow current decay. . . . . . . . . . . . . 995

6.14 Magnet performance with full quench heater failure. The nominal values are represented by lighter lines.

6.15 Quench load for different heater combinations (HF: high field, LF: low field, IL: inner layer).

6.16 Quench load and hot spot temperature after the failure of one, two, or three quench heater power supplies in a main dipole. Dashed lines: quench origin below failed heater. . . . . . . . . . . . . . . . . . . . 98

6.17 Quench load for different delays of the high field heater discharge from the nominal performance.

6.18 Magnet performance after missed quench. Up: quench load (light) and hot spot temperature (dark); Down: magnet voltage (light) and current (dark). . . . . . . . . 99

6.19 Hot spot temperature for different detection delays in a main dipole. . . . . . . . 100

6.20 Fault tree analysis (FTA) for the quench protection system of the LHC $13 \mathrm{kA}$ main magnets: Main Failure. . . . . . . . . . . . . . . . . . . . . . . . 101

6.21 Fault tree analysis (FTA) for the quench protection system of the LHC $13 \mathrm{kA}$ main magnets: False Quench. . . . . . . . . . . . . . . . . 101

7.1 General DQQDL layout with short-circuited wires and instrumented for checks. . . 104

7.2 Coherency check in a 2004 detector. Up: No failure, middle: one channel in Quench state, down: false quench generation. . . . . . . . . . . . . . . 105 
7.3 Quench test in a 2004 detector with one channel blind. . . . . . . . . . . . . . . 106

7.4 Markov diagrams for UM and FQ models of the DQQDL analog part. . . . . . . 106

7.5 Example of the impact of CT and QT tests on the reliability of a detector with 2003 redundant scheme (UM: Unprotected Magnet, MQ: Missed Quench, FQ: False Quench). The number in brackets makes reference to the test frequency. . . . 107

7.6 Reliability and availability with respect to MQ for two different quench detector topologies with yearly quench check . . . . . . . . . . . . . . . . . 108

7.7 Quench detector with redundant analog part $(k-o o-n) . \ldots 109$

7.8 Quench detector with redundant analog and logic parts $(k-o o-n d) . \quad \ldots 109$

7.9 Quench detectors in series redundancy $(k-o o-n$ and $k$-oo- $n) \ldots \ldots$. . . . . . . . 110

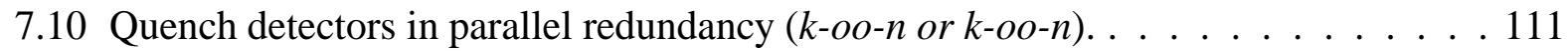

7.11 Probability of suffering less than one, two, three, and six missed quenches after 20 years in function of the total failure rate drift from the nominal value, $\lambda$, and the expected quench rate. 2oo2or 2002 detection scheme with yearly QT. . . . . . . . . 113

7.12 Probability of suffering less than one, two, three, and six missed quenches after 20 years in function of the total failure rate drift from the nominal value, $\lambda$, and the expected quench rate. 2oo2or 2002 detection scheme with monthly QT. . . . . . . . 114

7.13 Sensitivity of missed quenches after 20 years for $2 o o 2$ or $2 o o 2$ topology with yearly (left) and monthly (right) quench checks . . . . . . . . . . . . . . . 115

7.14 Pay-back period (in years) for $100 \%$ of the investment covered by CERN ( $\mathrm{r}=0.06$, $\Delta=2$ years) with first year operation of $50 \%$ (a), $70 \%$ (b), and $100 \%$ (c) of the scheduled time. . . . . . . . . . . . . . . . . . 116

7.15 Pay-back period (in years) for $50 \%$ of the investment covered by CERN ( $\mathrm{r}=0.06$, $\Delta=2$ years) with first year operation of $50 \%$ (a), $70 \%$ (b), and $100 \%$ (c) of the scheduled time. . . . . . . . . . . . . . . . . . . 116

7.16 Inner triplet circuit with location of the DQQDT quench detectors. . . . . . . . 118

7.17 One-channel DQQDI detector (PGA: Programmable Gain Amplifier, ADC: Analog Digital Converter, DSP: Digital Signal Processor). . . . . . . . . . . . . . . 119

7.18 Different detection topologies analysed for the DQQDI and DQQDT quench detectors. The dashed lines represents the commonly powered components (TMS: power supply).

7.19 Pareto analysis of false quenches in DQQDI and DQQDT detectors for 1002 detection scheme without redundant powering (left) and with shared power supplies (right). Monthly CT and yearly QT have been considered. . . . . . . . . . . . . . 122

7.20 Maximum number of missed quenches (95\% confidence) after 20 years in a DQQDG detector in front of the the total expected quenches per detector (monthly CT and yearly QT). . . . . . . . . . . . . . . . . . . 126

7.21 Pareto analysis of false quenches in DQQDG detectors for 1oo2 detection scheme without redundant powering (left) and with shared power supplies (right). Monthly CT and yearly QT have been considered. . . . . . . . . . . . . . . . . . . . . . . . . . . . . . . . . .

7.22 One-channel DQQDC quench detector. . . . . . . . . . . . . . . . . 128

7.23 Maximum number of missed quenches (95\% confidence) after 20 years in a DQQDC detector in function of the total expected quenches per detector (Monthly CT and yearly QT). . . . . . . . . . . . . . . . . . . . . . . . . 129

8.1 Quench heater circuits for the LHC main dipole (left) and quadrupole (right). . . . 131 8.2 Electrical design of the LHC quench heater power supplies. . . . . . . . . . . . 132 
8.3 Markov diagrams (state-space) for one branch with voltage checks (VT); BP: Beam Permit, BA: Beam abort. . . . . . . . . . . . . . . . . . . 136

8.4 Unprotected magnet reliability for a single dipole magnet. . . . . . . . . . . . . 138

8.5 Number of unprotected dipoles with a 95\% confidence level. . . . . . . . . . . 138

8.6 Markov diagram with discharge test (PP:Power Permit, PA:Power Abort, DT:Discharge Test). . . . . . . . . . . . . . . . . . . . 139

8.7 Capacitor and thyristor reliability terms for a single HPS and for different maintenance policies. . . . . . . . . . . . . . . . . . 140

8.8 Left: Capacitor and thyristor reliability terms for a single HPS. Right: Unprotected magnet and lost magnet reliability for the 1232 LHC dipoles. . . . . . . . . . . . . 141

8.9 Sensitivity of maximum number of lost dipoles (90\% confidence level) to component failure rate and quench rate with maintenance policies $1 y$ DT $p 0$ (left) and $1 y$ DT pl (right). . . . . . . . . . . . . . . . . . . . 143

8.10 Sensitivity of maximum number of lost quadrupoles ( $90 \%$ confidence level) to component failure rate and quench rate with maintenance policies $1 y$ DT pO (left) and $1 y$ DT $p l$ (right). . . . . . . . . . . . . . . . . . . 143

9.1 Switches and extraction resistors topologies for $13 \mathrm{kA}$ (left) and $600 \mathrm{~A}$ (right) LHC circuits. . . . . . . . . . . . . . . . . . . 146

9.2 $600 \mathrm{~A}$ and $13 \mathrm{kA}$ controllable fuses for the LHC energy extraction facilities.. . . . . 149

9.3 Failure probability of the $13 \mathrm{kA}$ energy extraction facility after 20 years with yearly repairs (left) and repairs after each demand (right). . . . . . . . . . . . . . 153

9.4 Failure probability during 20 years of the $13 \mathrm{kA}$ energy extraction facility with an implemented controlled fuse. . . . . . . . . . . . . . . . . . . 154

9.5 Safety Integrity Level of the $13 \mathrm{kA}$ energy extraction facility without fuse for yearly repairs (left) and repairs after each demand (right) . . . . . . . . . . . . . 155

9.6 Failure probability of the 600 A energy extraction facility after 20 years with yearly repairs (left) and repairs after each demand (right). . . . . . . . . . . . . . . 157

9.7 SIL for the 600 A energy extraction system without third fuse (up) and with it (down) for with maintenance carried out after each request (right) or yearly (left). . 159

10.1 Relationships between the interlock and the machine subsystems. The systems dedicated to protection are within the grey area. . . . . . . . . . . . . . 162

A.1 Gain stability of the AD620 (left) and INA141 (right) isolation amplifiers. . . . . 173 


\section{List of Tables}

1.1 Some machine parameters $[3] \ldots \ldots \ldots \ldots \ldots$

3.1 The main parameters of the LHC dipole magnets [18]. . . . . . . . . . . . 26

3.2 Main parameters of the MQ magnets [18] . . . . . . . . . . . . . . . 27

3.3 Some parameters of the main dipole (MD) and quadrupole (MQ) energy extraction system. . . . . . . . . . . . . . . . . . . . . . . . . . . . . . . 35

3.4 Protection Requirements for the LHC corrector circuits [33] . . . . . . . . . . . 37

6.1 Particle spectra close to the LHC dipole surface [61] . . . . . . . . . . . . . 78

6.2 Cable parameters of the $13 \mathrm{kA}$ LHC dipole. . . . . . . . . . . . . . . 88

6.3 Quench and protection performance parameters of QUABER dipole model. . . . . 89

6.4 Delay times for a full current dipole (see Fig. 6.8) . . . . . . . . . . . . . . . 90

6.5 Failures in the energy extraction banks: total resistance of the circuit $R_{\text {total }}$, current decay time constant $\tau$ and energy dissipated in the diode busbars $E_{b b}$. . . . . . 95

7.1 Failure rate data for DQQDL components. . . . . . . . . . . . . . . . 107

7.2 DQQDL results with monthly CT and yearly QT. . . . . . . . . . . . . . . . 109

7.3 DQQDL results with monthly $\mathrm{CT}$ and yearly QT . . . . . . . . . . . . 110

7.4 DQQDL results with monthly $\mathrm{CT}$ and yearly QT . . . . . . . . . . . . 111

7.5 DQQDL results with monthly $\mathrm{CT}$ and yearly QT . . . . . . . . . . . 111

7.6 Comparison between the two topologies. Missed quench reliability for all the dipole quench detectors, expected missed quenches with a 95\% confidence level for yearly and monthly QT and mean of expected false quenches for monthly CT. . 112

7.7 Mean values for the two studied topologies to reduce false quenches due to powering. 115

7.8 Expected pay-back period of the investment needed to double the DQQDL power supplies, in function of $C_{s}\left(10^{3} C H F.\right) \ldots \ldots \ldots \ldots$

7.9 Insertion and inner triplet circuits of the LHC . . . . . . . . . . . . . . . 119

7.10 Failure rate data for the components of the DQQDI and DQQDT detectors. . . . . 120

7.11 DQQDI and DQQDT reliability and expected failures (95\% confidence level) after 20 year with monthly CT and yearly QT. . . . . . . . . . . . . . . . . . . . . . . . . 22

7.12 Quench rate of the LHC corrector circuits. . . . . . . . . . . . . . . . . . . . . . . . . . . . . . . . . . . . . .

7.13 Failure cost of the LHC corrector circuits. . . . . . . . . . . . . . . . . 125

7.14 DQQDG reliability and expected failures (95\% confidence level) after 20 years with monthly CT and yearly QT. . . . . . . . . . . . . . . . . . . . . . . . . . . . . . . .

7.15 Failure rate data for DQQDC components. . . . . . . . . . . . . . 128

7.16 DQQDC reliability and expected failures (95\% confidence level) after 20 year with montly CT and yearly QT. . . . . . . . . . . . . . . 129

8.1 Component reliability data from MIL-HDBK-217F . . . . . . . . . . . 133 
8.2 LHC quench power supplies and quench rates. . . . . . . . . . . . . . . 135

8.3 Reliability results for dipole and quadrupole quench heater power supplies after 20 years. HPS: Lost Power Supplies (needed spare), UM: Unprotected Magnets, LM: Lost Magnets, $R_{L M}$ probability of not loosing any magnet after 20 years of LHC operation. . . . . . . . . . . . . . . . . . . . . . 142

9.1 Mean Time to Failure (MTTF) of the fuses used in the continuous model. . . . . . 152

9.2 Failure probability after 20 years of the $13 \mathrm{kA}$ energy extraction with yearly maintenance $\left(\lambda_{s} \equiv \lambda_{\text {switch }}\right)$. . . . . . . . . . . . . . . . . . . . 154

9.3 Failure probability after 20 years of the $13 \mathrm{kA}$ energy extraction with maintenance after opening demand $\left(\lambda_{s} \equiv \lambda_{\text {switch }}\right) \ldots \ldots \ldots \ldots \ldots \ldots \ldots \ldots \ldots \ldots$

9.4600 A energy extraction facilities. . . . . . . . . . . . . . . . . . 156

9.5 Failure probability after 20 years of the $600 \mathrm{~A}$ energy extraction with yearly maintenance $\left(\lambda_{s} \equiv \lambda_{\text {switch }}\right)$. . . . . . . . . . . . . . . . . . 157

9.6 Failure probability after 20 years of the 600 A energy extraction with maintenance after opening demand $\left(\lambda_{s} \equiv \lambda_{\text {switch }}\right) \ldots \ldots \ldots \ldots \ldots \ldots \ldots \ldots$

10.1 Hardwired signals between QPS, power converters and Powering Interlock Controllers generating a power abort. . . . . . . . . . . . . . . . . . . . . . 164

10.2 QPS Channels to the powering interlock and global reliability (spurious triggered not considered) . . . . . . . . . . . . . . . . . . . . . . 166

10.3 Global QPS maintainability strategy. . . . . . . . . . . . . . . . . . . . 167

A.1 Influence of the radiation to the output voltage of different voltage references. . . . 171

A.2 Linear voltage regulators submitted to radiation tests. . . . . . . . . . . . . 172

A.3 Switch mode power supplies. . . . . . . . . . . . . . . . . . . . . 173

A.4 ADuC812 data acquisition system. . . . . . . . . . . . . . . . . . . . . 174

A.5 Irradiation tests of the quench heater power supplies. . . . . . . . . . . . 175

C.1 SIL with low demand mode of operation (IEC-61508) . . . . . . . . . . . . . . 179

C.2 SIL with high demand or continuous mode of operation (IEC-61508). . . . . . . 179

C.3 Minimum Safety Integrity Level required (IEC-61508) . . . . . . . . . . . . . . . 179

C.4 Frequency categories. . . . . . . . . . . . . . . . . . . . . . . 180

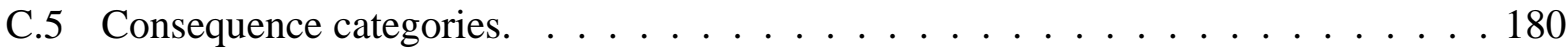




\section{Acknowledgements}

I would like to thank Prof. Francisco Calviño for his initiative, which made possible doing my Diploma and Doctoral Theses at CERN, and his constant support during these four years.

I have to express my sincere thanks to Félix Rodríguez Mateos. He has been my supervisor and closest colleague. His intelligent leadership together with his deep knowledge of the LHC have made my effort much easier. I hope the installation of the LHC and future projects will allow me to keep enjoying both his professional skills and his friendship.

I would also like to express my gratitude to Dr. Reiner Denz, for his continuous and valuable support during the development of this thesis. It has been a pleasure and a privilege to work together within so many projects: irradiation tests, String-2 installation, quench detectors and quench heater power supplies.

The analysis of the energy extraction facilities would have not been possible without the help of Knud Dahlerup-Petersen. My interaction with him has been extremely dynamic and fruitful.

I have been Doctoral Student within the AT/MEL group (formerly LHC/ICP). I wish to thank all its members. It has been a privilege to start my professional career in such a kind environment.

Special thanks go to the String-2 team, specially to Davide Bozzini, Jose Luis Gómez-Costa, Tomasz Ladzinski and the Project Leader Roberto Saban. I will never forget the long and interesting installation and commissioning days.

I am much obliged to my friend Marco Calvi. Our endless discussions have been essential for the development of my theoretical models. Thanks also to Dr. Ruediger Schmidt and Dr. Bernd Dehning, whose valuable comments and ideas remarkably improved the quality of my work.

This doctoral thesis is the most important step of my student career. I want to remember and sincerely thank all the professors in Valldemia who led my first steps into the world of Science. Special thanks go to my High School physics teacher Pilar Izquierdo.

And, of course, thanks to Federico, Alberto, Luis, Stefano, Mirko, Georgina, Jose, Juan, Álex, Iván, Bobo, Cocco, Giovanna, Tatiana, Jan, Christos, Gemma, Andrea, Davide, Chiara, Mario, Elena, Sonia, Stefano, Paolo, Fabrizio, Florian, Laura, Gianluca, Giuseppe, Andrés, Rocío and Ubaldo. I love you guys.

No quiero acabar sin agradecer a mi familia su constante apoyo. Especialmente a mis padres Antonio y María Fuensanta, y a mis hermanas Rocío y Estrella, su infinito cariño y paciencia han sido el motor de éste y todos mis proyectos.

A mi amigo David, que lo es hasta más allá del alma. A Xevi, por tantas horas juntos en bibliotecas y bares. Gracias a Germán, Pedro, Ságar, Dani, Iván, Javi, Mireia, Vero, Cristina, Anabel, Nuria, Óscar, Angie, Montse... estar lejos de ellos ha sido lo peor de estos años.

Gracias Esther, mi cómplice, por compartir conmigo todos los días y noches de alegrías y enfados que esta tesis me ha dado. Tu amistad es mi mayor tesoro. Esta tesis es tan tuya como mía.

Y gracias Raquel por tu aliento, tus sueños, tus besos... mi envenenada medicina. 